\title{
IntechOpen
}

\section{Advances in Quantum Communication and Information}

Edited by Francisco Bulnes, Vasilios N. Stavrou, Oleg Morozov and Anton V. Bourdine 



\title{
Advances in Quantum Communication and Information
}

\author{
Edited by Francisco Bulnes, \\ Vasilios N. Stavrou, Oleg Morozov \\ and Anton V. Bourdine
}



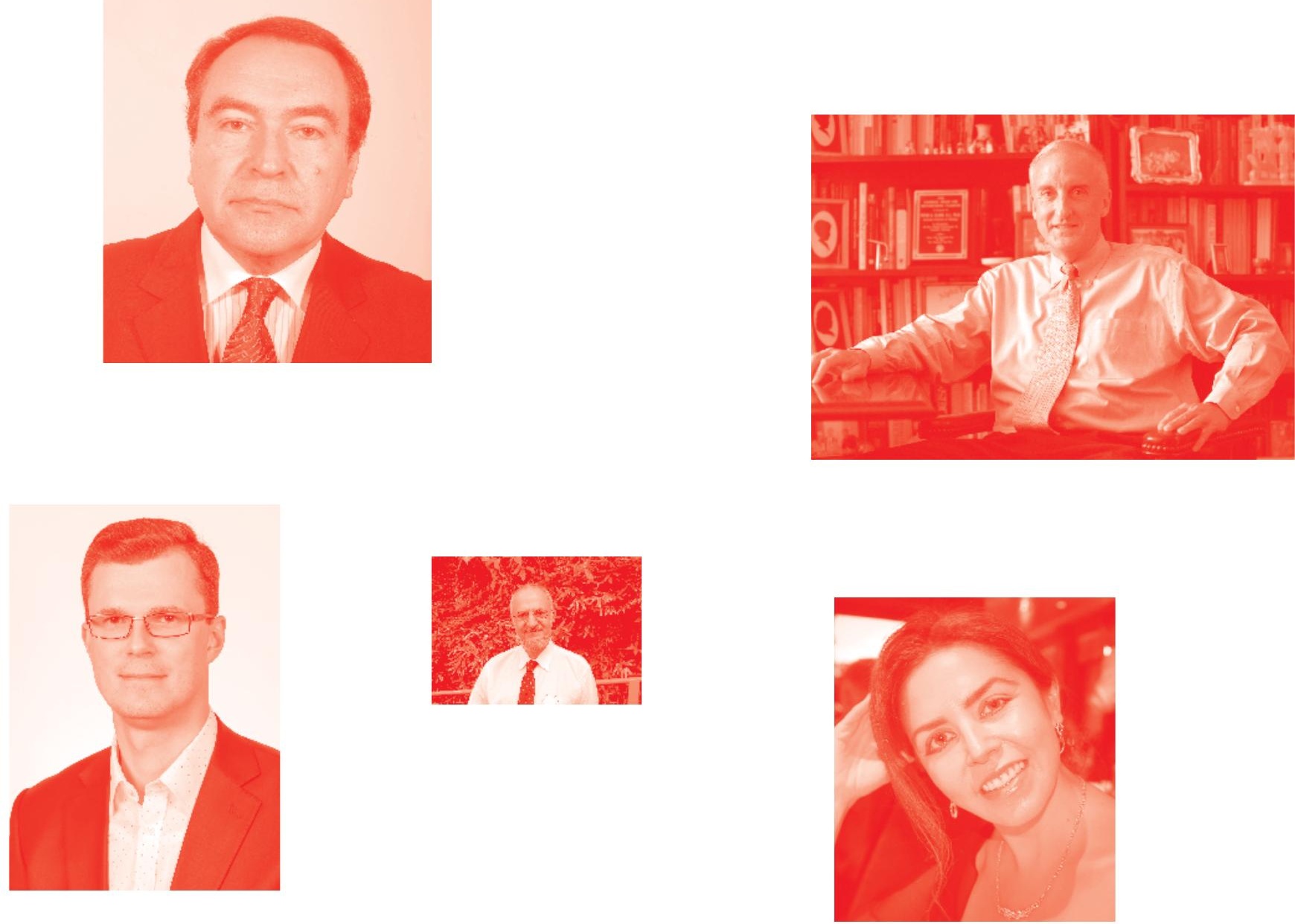

Supporting open minds since 2005
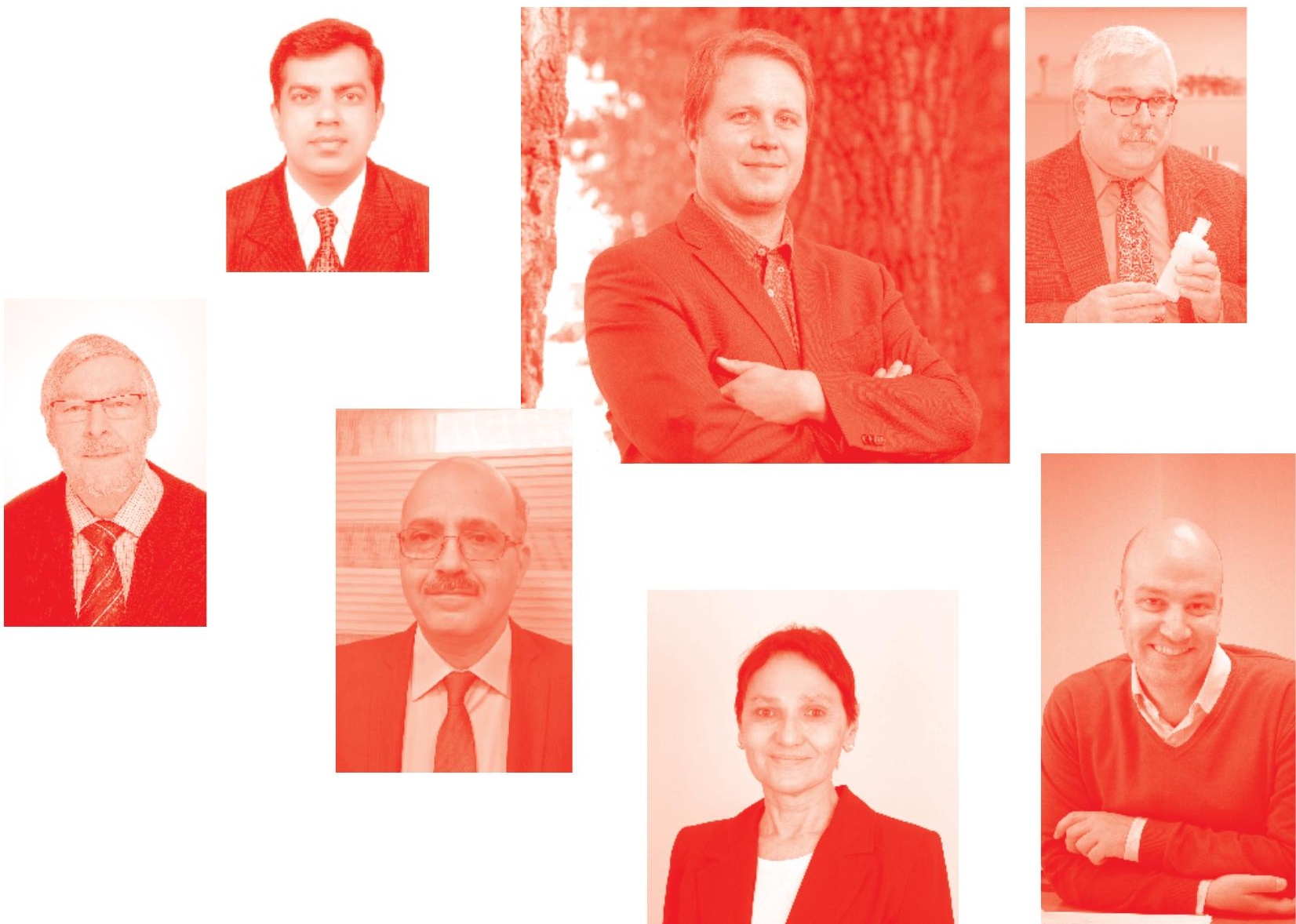
Advances in Quantum Communication and Information

http : //dx . doi . org/10.5772/intechopen. 82506

Edited by Francisco Bulnes, Vasilios N. Stavrou, Oleg Morozov and Anton V. Bourdine

\section{Contributors}

Rene Steijl, Mustapha Ziane, Morad El Baz, Rigui Zhou, Jia Luo, Dan Nguyen, Daniel Nolan, Nicholas Borrelli, Francis Yu, R Anantharaj, Sai Saravanan A.V, Aghaddin Mamedov, Francisco Bulnes, Ismail Gassoumi

๑) The Editor(s) and the Author(s) 2020

The rights of the editor(s) and the author(s) have been asserted in accordance with the Copyright, Designs and Patents Act 1988. All rights to the book as a whole are reserved by INTECHOPEN LIMITED . The book as a whole (compilation) cannot be reproduced, distributed or used for commercial or non-commercial purposes without INTECHOPEN LIMITED's written permission. Enquiries concerning the use of the book should be directed to INTECHOPEN LIMITED rights and permissions department (permissions@intechopen.com).

Violations are liable to prosecution under the governing Copyright Law

\section{(cc) BY}

Individual chapters of this publication are distributed under the terms of the Creative Commons Attribution 3.0 Unported License which permits commercial use, distribution and reproduction of the individual chapters, provided the original author(s) and source publication are appropriately acknowledged. If so indicated, certain images may not be included under the Creative Commons license. In such cases users will need to obtain permission from the license holder to reproduce the material. More details and guidelines concerning content reuse and adaptation can be found at http : //www . intechopen . com/copyright-policy . html .

\section{Notice}

Statements and opinions expressed in the chapters are these of the individual contributors and not necessarily those of the editors or publisher. No responsibility is accepted for the accuracy of information contained in the published chapters. The publisher assumes no responsibility for any damage or injury to persons or property arising out of the use of any materials, instructions, methods or ideas contained in the book.

First published in London, United Kingdom, 2020 by IntechOpen IntechOpen is the global imprint of INTECHOPEN LIMITED, registered in England and Wales, registration number: 11086078 , 7th floor, 10 Lower Thames Street, London,

EC3R 6AF, United Kingdom

Printed in Croatia

British Library Cataloguing-in-Publication Data

A catalogue record for this book is available from the British Library

Additional hard and PDF copies can be obtained from orders@intechopen.com

Advances in Quantum Communication and Information

Edited by Francisco Bulnes, Vasilios N. Stavrou, Oleg Morozov and Anton V. Bourdine

p. cm.

Print ISBN 978-1-78985-267-7

Online ISBN 978-1-78985-268-4

eBook (PDF) ISBN 978-1-78985-247-9 


\section{We are IntechOpen, \\ the world's leading publisher of Open Access books}

\section{Built by scientists, for scientists}

\section{$4,600+$}

Open access books available

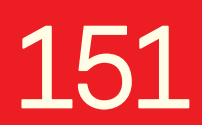

Countries delivered to

\section{$120,000+$}

International authors and editors

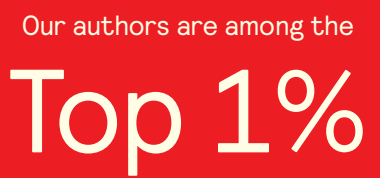

most cited scientists

Contributors from top 500 universities
$135 \mathrm{M}+$

Downloads
1200

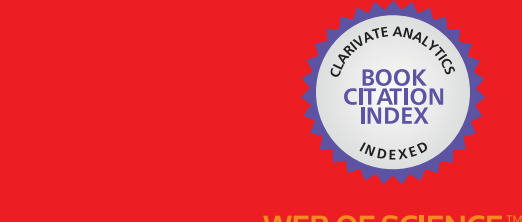

Selection of our books indexed in the Book Citation Index in Web of Science ${ }^{\mathrm{TM}}$ Core Collection (BKCI)

\section{Interested in publishing with us? \\ Contact book.department@intechopen.com}

Numbers displayed above are based on latest data collected.

For more information visit www.intechopen.com 



\section{Meet the editors}

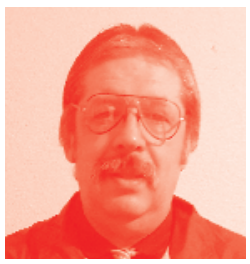

Dr Francisco Bulnes received his $\mathrm{PhD}$ in Mathematical Sciences from IM/UNAM, 2004. He is the Director of International Advanced Research in Mathematics and Engineering Centre in Mexico (IINAMEI), since 2015. He is the Editor-in-Chief of two Journals of Mathematics, one in USA, and the other in India. Dr Bulnes is a member of various international committees of science and mathematics and a reviewer of British journals of mathematics and physics indexed in SCOPUS. Dr Bulnes has been the Head of Research Department, TESCHA, 2009. He has written numerous papers (more than 100 ) or articles on mathematics and physics research journals, and he is the author of more than twenty books (math monographs and specialized book chapters in theoretical physics and advanced electronics research). Dr Bulnes is recognized and famous in East Europe, Asia, and the Arab continents. He has many theories, theorems, and math objects under his name. He has received various Doctor Honoris Causa by universities, NGOs, and GOs. His biography has been included in the GMW book, published in the IBC, Cambridge, UK, also in China and India. Dr Bulnes is also a distinguished member (JCFM) of the Czech Republic Mathematics Society. He obtained two Post-doctorates in Cuba and Russia in mathematics.

Dr V. N. Stavrou is currently an Adjunct Member at the Hellenic Naval Academy, Piraeus, Greece. He received a MSc and a PhD in Theoretical Solid-state Physics from the University of Essex in England, in 1995 and in 1999 respectively. He has held postdoctoral positions at the following research institutions: a) Deutsche Forschungsanstalt fuer Luft und Raumfahrt e.V (German Aerospace Research Center) in Germany, b) Helsinki University of Technology, c) State University of New York (SUNY) at Buffalo, USA, and d) University of Iowa, USA. He is specifically interested in researching the electronic, optical, and lattice properties of semiconducting low dimensional structures (quantum dots and quantum wells amongst others). These properties are of special importance for quantum computing architecture and laser technology. He has published his research work in reputable journals related, among others, to quantum computing, spintronics, decoherence in quantum dots, diluted magnetic semiconductors, and phonon models in low dimensional structures.

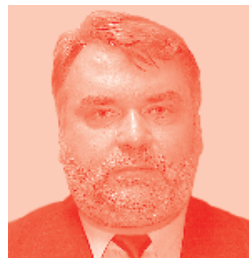

Oleg Morozov is a full professor at the Kazan National Research Technical University (KNRTU-KAI), Head of Radiophotonics and Microwave Technologies Department, and Director of the R\&D Institute of Applied Electrodynamics, Photonics and Living Systems. He leads research on microwave photonics, fiber optic sensors and communications, and the transfer of optical methods into microwave sensors and technologies. He has over 100 papers and 10 monographs concerning principles of tandem amplitude-phase modulation of microwave and optical carriers and its application in a wide spectrum of technical and living systems. Over the past five years, his interests have 
been in the field of quantum communications, in particular, the quantum key distribution on frequency-coded subcarriers. Research in this area is carried out jointly with the Kazan Quantum Center of KNRTU-KAI. Oleg G. Morozov is an Honorary Worker of Higher Education, Fellow of International Academy of Telecommunications, Senior Member of OSA and SPIE, regular member of Optical Society (ROS), EOS, and IEEE.

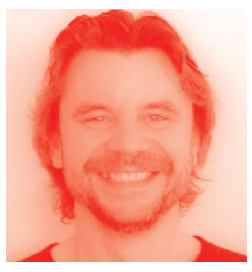

Anton Bourdine (b. 1977) embarked on a career in the field of fiber optics in telecommunications in 1996 in LLC R\&D Co.

"CAM" (Samara, Russia), where he was involved as a technician and further as an engineer in the installation of aerial and underground fiber optic links. He earned his PhD from the Povolzhskiy State University of Telecommunications and Informatics (PSUTI, Samara, Russia) on November 2002 and received his degree of Doctor of Technical Sciences after defending his doctorate dissertation on December 2013. Since then he has been a professor at the Department of Communication Lines of PSUTI. Anton Bourdine is a fellow of OSA and SPIE. He is an author and co-author of more then 250 published scientific papers, more than 30 patents, and 2 monographs. 


\section{Contents}

Preface

Section 1

Introduction

Chapter 1

Introductory Chapter: Advanced Communication and

Nano-Processing of Quantum Signals

by Francisco Bulnes

\section{Section 2}

Advanced Research in Hypercomplex Platforms in

Quantum Communication

Chapter 2

A Novel Quantum Steganography Scheme Based on ASCII by Ri-Gui Zhou and Jia Luo

Chapter 3

Quantum Algorithms for Fluid Simulations

by René Steijl

Chapter 4

Non-Gaussian Entanglement and Wigner Function

by Mustapha Ziane and Morad El Baz

Section 3

Praxiological Studies of Quantum Communication

Chapter 5

Quantum Walks in Quasi-Periodic Photonics Lattices

by Dan Trung Nguyen, Daniel A. Nolan and Nicholas F. Borrelli

Section 4

Applications and Technologies

Chapter 6

Design of 4-Bit 4-Tap FIR Filter Based on Quantum-Dot Cellular Automata (QCA) Technology with a Realistic Clocking Scheme by Ismail Gassoumi, Lamjed Touil, Bouraoui Ouni and Abdellatif Mtibaa 
DFT Study on Interaction of Estrone and Imidazolium-Based

Hydrophobic Ionic Liquids

by Sai Saravanan Ambi Venkataramanan and Ramalingam

Anantharaj

Section 5

Quantum Mechanisms and Meta-Heuristics Concepts About

Quantum Communication

Chapter 8

Schrödinger's Cat and His Timeless $(t=0)$ Quantum World by Francis T.S. Yu

Chapter 9

What Is "Wrong" with Current Theoretical Physicists?

by Francis T.S. Yu

Chapter 10

The Hot Disputes Related to the Generation of a Unified Theory Combining the Outcomes of ER and EPR Papers

by Agaddin Mamedov 


\section{Preface}

Research in quantum communication will have fundamental implications for teleportation, advanced communication, and artificial intelligence where their processes happen in the space of more dimensions than the fourth, which includes the quantum field theory of the phenomenology of these processes as a base to its possibility. Likewise, the quantum communication and their information phenomena require other ways of thinking to develop new technology and to program its devices, the development of quantum computation, possible different platforms of this quantum communication as to their applications. This book is a compilation of research in quantum communication by different international, recognized scientists, who are authorities in this area. The book is divided in five sections. The first section is an introduction chapter, which establishes the fundamental quantum field theory of quantum communication and introduces new concepts such as link-waves, tranceptors, and quantum chain to intertwining. The second section is dedicated to the advanced research in quantum computation and their platforms in the complex systems of quantum communication This section introduces new principles such as Non-Gaussian entanglement, new quantum algorithms to fluids (which could be very important to plasmon management), hyperfluidity, programming of fluids, and the quantum steganography scheme as a platform to quantum communication. The third section establishes a prospective study of quantum walks on photonic lattices in the photonic process that could be given in the quantum communication processes. The first four sections explain some aspects of the technology in quantum communication. The fifth section discusses some heuristic and theoretical problems of QED and quantum mechanics that can solve or re-interpret certain paradoxes useful in quantum communication and quantum field theory aspects.

Dr. Francisco Bulnes

Professor, Iinamei, Director, Mexico Research Department in Mathematics and Engineering, TESCHA, Mexico

Dr. Vasilios N. Stavrou Hellenic Naval Academy, Greece

Dr. Oleg Morozov

Kazan National Research Technical University, n.a. A.N. Tupolev-KAI, Russia

Dr. Anton V. Bourdine

Volga State University of Telecommunications and Informatics, Russia 

Section 1

\section{Introduction}





\title{
Introductory Chapter:
}

\section{Advanced Communication and Nano-Processing of Quantum Signals}

\author{
Francisco Bulnes
}

\section{Introduction}

In the usual communication processes, the transmission of electromagnetic waves is realised with limitations given due to the scattering in continuum media, interference for appearing of other waves and signals, and also pollution by free electrons, molecular absorption of the air, or a defecting routing of the TEM and TMM, as well as their secondary modes, the latter in the case of the optical fibre. Even nonthinking is the effective transmission through impermeable media and nonpermissible or invasive media such as the communication through ambient with continuum media, saturated continuum media in communications or in the presence of multi-radiative objects of big density, etc.

Likewise, the continuum transmission media produces a limiting whose dependence of the macroscopic characteristics of the space-time do that the electromagnetic fields suffer refraction and reflection due to the substance of the proper media. For eliminate this direct action of media in the signal transmission, it is necessary that our signal communication concept does not depend on the media characteristics, even that are not 'accessible or available' in the dimension of the usual space-time where we have placed the continuum media phenomena. For example, the gravitation in the macroscopic sense has interference in the ordinary communication where field observables of an Einstein space (as could be the curvature and torsion of the space-time, seen as gravitational observable effects or of electromagnetic scattering) act as distortion waves to produce a field radiation due the background in the universe. Then the signal transmission must have the invariance of the electromagnetic fields but with quantization of these fields, in a space that topologically is viable to their transmission [1].

Considering the quantum field theory [2] (QFT) with their second duality principle, that is to say, the duality wave/field applied to certain fermions and bosons, we can give a good fundament on the possible behaviour of these particles and their action with the communication intention [3]. However, it is necessary to consider the invariance of the Maxwell equations in quantum electrodynamics (QED) [1, 4], which we can design a form of quantum communication that establishes new paradigms of information transmission through quantum waves. However, in this new communication process, the intertwining concept of the particles is required [5] that will transmit the information via a chain of photons using gauge bosons as supports (selectors or linkers) of the electrodynamic space to their quantum level.

The material part of the transmission is generated in the transceptors through their solid state. Therefore, using electrons as solid-state source to generate a source 
of photons through a process derived from certain spintronic devices as dots, magnetons, etc., the bosons can be conformed to the information of the communication in waveform and the plasmons as a quantum media of transmission of these information waves. The gauge bosons also will be necessary as transmission nodes. If we want to obtain voice communication, this will be obtained introducing phonons [5] in the photonic wave. We establish some fundamental precisions using quantum mechanics to explain those particles intertwining. We must consider that to that this intertwining happens, are necessary more dimensions that Minkowski space-time dimension.

We consider a particle system $p_{1}, p_{2}, \ldots$ in a space-time $M \cong R^{4}$. Let $x(t) \in \Omega(\Gamma) \subset R^{3} I_{t}$, a trajectory, which predetermines a position $x \in R^{3}$, for all time $t \in I_{t}$. Also we consider the field $X$, which infiltrates their action to whole space of points $x_{1}(t), x_{2}(t), x_{3}(t), \ldots \in \Omega(\Gamma)$, predetermining the points $\varphi_{i}\left(x_{i}(t)\right)$, that are field particles of the field $X$ and evaluated in the position of every particle. In each point, a defined force exists given by the action $\mathfrak{I}$, of $X$, along the geodesic $\gamma_{t}$, and determines direction by their tangent bundle given for $\operatorname{TX}^{1}(\Omega(\Gamma))$; that is to say, the field gives direction to every field particle $\varphi_{i}$, having their tangent bundle has a spinor bundle $S$, where the field $X$ comes given as $X=\left.\sum_{i} \varphi^{i} \frac{\partial}{\partial \varphi^{i}}\right|_{\left(x^{i}, \varphi^{i}\right)}, \forall \varphi_{1}, \varphi_{2}$, $\varphi_{3}, \ldots \in X^{1}$, on every particle $p_{i}=x_{i}(t),(i=1,2,3, \ldots)$. Then a direct intention is the map or connection $\nabla^{\mathfrak{I}}: T \Omega(\Gamma) \rightarrow T^{1}(\Omega(\Gamma))$, ( $\left.\simeq T * M\right)$, with the rule of correspondence $\left(x_{i}, \partial_{t} x^{i}\right) \mapsto\left(\varphi^{i}, \partial_{\mu} \varphi^{i}\right)$, which produces one ith-spinor field $\varphi^{i}$, where the action $\mathfrak{I}$, of the field $X$, infiltrates and transmits from particle to particle in whole space $\Omega(\Gamma)$, using a configuration given by their Lagrangian $L$ (conscience operator), along all the trajectories of $\Omega(\Gamma)$. Then of a sum of trajectories, $\int D_{F}(x(t))$, one has the $\operatorname{sum} \int d(\varphi(x))$, on all the possible field configurations $C_{n, m}$.

We can to extend these to whole space $\Omega(\Gamma) \subset M$, on all the elections of possible paths whose statistical weight corresponds to the determined one by the intention of the field, and realising the integration in paths for an infinity of particles-fields in $T \Omega(\Gamma)$, is had that

$$
\begin{aligned}
I\left(\varphi^{i}(x)\right) & =\int_{T \Omega(\Gamma)} \omega(\varphi(x))=\lim N \underset{\delta s \rightarrow 0}{\rightarrow} \infty \frac{1}{B} \int_{-\infty}^{+\infty} \frac{d \varphi_{1}}{B} \cdots \int_{-\infty}^{+\infty} \frac{d \varphi_{n}}{B} \cdots \\
& =\prod_{i=1}^{\infty} \int_{-\infty}^{+\infty} e^{i \Im\left[\varphi^{i}, \partial_{\mu} \varphi^{i}\right]} d \varphi^{i}(x(s))
\end{aligned}
$$

where $B=\left[\frac{m}{2 \pi i \delta s}\right]^{1 / 2}$ is the amplitude of their propagator. Then we have the corresponding Feynman integral of the volume form $\omega(\varphi(x))$, obtaining the real path of the particle (where we have chosen quantized trajectories, that is to say, $\int d(\varphi(x))$. But this superposition of paths is realised under an action whose corresponding energy Lagrangian is $\omega(\varphi(x))=\mathfrak{I}_{\xi(x)} d(\varphi(x))$.

Then to a configuration on the space-time $M$, given for $C(M)$, in a space-time region where there have been interfered paths in the experiment given by multiple split, given for $\Omega(M)$, we have the pairing $\int: C(M) \times \Omega *(M) \rightarrow R$, where $\Omega *(M)$ is some dual complex ('forms on configuration spaces'), and then the 'Stokes theorem' holds

$$
\int_{\Omega \times C} \omega=<\mathfrak{I}, d \omega>
$$


then Eq. (1) can be written as (to $m$-border points and $n$-inner points)

$$
\begin{aligned}
\int \mathfrak{I}(\varphi(x)) d \varphi(x) & =\int \Im_{q} d \varphi_{1^{m_{1}}} \ldots d \varphi_{n^{m_{n}}} \ldots \\
& =\int\left(\int\left(\int \Im d \varphi_{1^{m_{n}}}\right) \ldots d \varphi_{n^{m_{1}}}\right) \ldots
\end{aligned}
$$

This defines an infiltration in the space-time due to the action $\mathfrak{I}$ that happens in the space $\Omega \times C$, to each component of the space $\Omega(\Gamma)$, with energy conservative principle expressed for the Lagrangian $\omega$, of Eq. (2). Likewise, in Eq. (3), the integration of the space is realised with the infiltration of the time, integrating only spinor elements of the field.

\section{Quantum communication link waves}

Let $\mathbb{M}$, be the space-time constituted by the particles $x_{1}(t), x_{2}(t), x_{3}(t), \ldots$, whose states $\varphi_{1}, \varphi_{2}, \varphi_{3}, \ldots$ are such that satisfies Eq. (3); then the information is transmitted like the quantum wave $\phi$, of the state $\varphi$, replaced with the state $\varphi^{\prime}{ }_{i},(i=1,2,3, \ldots)$, in the infinite homomorphism (which is of the type $\phi(n * m)=\phi(n) \phi(m))$ :

$$
\phi\left(t_{\varphi^{\prime} 1} * t_{\varphi^{\prime} 2} * t_{\varphi^{\prime} 3} * t_{\varphi^{\prime} 4} \cdots\right)=\phi_{\varphi_{1}}\left(t_{\varphi^{\prime} 1}\right) \phi_{\varphi_{2}}\left(t_{\varphi^{\prime} 2}\right) \phi_{\varphi_{3}}\left(t_{\varphi^{\prime} 3}\right) \phi_{\varphi_{4}}\left(t_{\varphi_{4}^{\prime} 4}\right) \cdots
$$

where the transmission of the quantum wave is realised on the spinor space (see spinor technology [6]) of $t_{\sigma}$ and where $t_{\sigma}$ is the intertwining technology that is created in the class $\sigma$.

The states of distinguishable particles that are bosons or fermions realise the arrangement that eliminates an infinity of the states that by their sum of spins are annulled, remaining only those that realise an effective action. These are annulled between the perturbed states and those that are affected by scattering. We consider again the space of configuration $C_{n, m}$, equivalent to the complex given for $C(M)$, which can be thought as composed for $n$-hypercubes $U$, defined by $000 \ldots 0$ boxes. Then we can define a net of paths that will be able to establish routes of organised transformations on diagrams of Feynman type (with path integrals with actions given by $O_{c}$ and path integrals as Eq. (1)). Likewise the ideal route of the intention is established, considering the action in every node of the net.

Then these arrangements can happen in the nets designed on a field of particles that can be arranged in $0000 \ldots 0$ boxes [7], where the action can be calculated in a point (node of the crystalline net of a field [7]) corresponding to the $n$ - states of energy $\varphi_{i}(i=1,2,3, \ldots, n)$, having the superposition $\mathrm{n}$ the node given for

$$
\oint[x]_{U}=\int_{000 \cdots 0-\text { boxes }} d z^{n} \phi\left(X^{\alpha_{1}} W_{\alpha_{1}^{\prime}}\right) \phi\left(X^{\alpha_{2}} W_{\alpha_{2}^{\prime}}\right) \cdots \phi\left(X^{\alpha_{n}} W_{\alpha_{n}^{\prime}}\right)
$$

In the quantum zone, the quantum particles field is permanent and interminable, since matter and energy are equivalent and the electrons are interminable and thus the photon production also. What gets worn is that there are the linkages between atoms which can weaken or get lost for the absence of a transmission of the states of suitable energy (routes given by path integrals). Infiltrating the intention on every path $\gamma$ and under the condition of permanent field given by the operators $O_{c}$, the transmission of the states will be revitalised by every node, transmitting the 
same information about every $0000 \ldots 0$ box, we will call this characteristic an intentionality [8]. However, every particle with regard to others takes their corresponding position, since they all have the same infiltrated intention, by what the synergic action is realised.

\section{Author details}

Francisco Bulnes

Research Department in Mathematics and Engineering, TESCHA, IINAMEI (Investigación Internacional Avanzada en Matemáticas e Ingeniería), Mexico

*Address all correspondence to: francisco.bulnes@tesch.edu.mx

\section{IntechOpen}

(C) 2019 The Author(s). Licensee IntechOpen. This chapter is distributed under the terms of the Creative Commons Attribution License (http://creativecommons.org/licenses/ by/3.0), which permits unrestricted use, distribution, and reproduction in any medium, provided the original work is properly cited. (c) BY 


\section{References}

[1] Bulnes F, Bulnes FH, Hernández E, Maya J. Diagnosis and spectral encoding in integral medicine through electronic devices designed and developed by path integrals. Journal of Nanotechnology in Engineering and Medicine. 2011;2: 021009

[2] Feynman RP. QED: The Strange Theory of Light and Matter. New Jersey, USA: Princeton University Press; 2006

[3] Schwinger J. Particles, Sources and Fields. New York, USA: Advanced Book Program, Perseus Books; 1998

[4] Bulnes F. Mathematical nanotechnology: Quantum field intentionality. Journal of Applied Mathematics and Physics. 2013;1:25-44. DOI: $10.4236 /$ jamp.2013.15005

[5] Bulnes F. Photonic chains of wavelinks to the quantum communication. Journal on Photonics and Spintronics. 2015;4(3):10-14

[6] Guderian D. Weyl indices in the spinor frame and their products with technological implications. Journal on Photonics and Spintronics 2014;3(1):4-11

[7] Bulnes F. Teoría de Algoritmos para la Maestría de Informática Aplicada, Universidad de las Ciencias Informáticas (UCI); Habana, Cuba; Postdoctoral Project (Aplicaciones de la Teoría Infinita de Lie y sus Aplicaciones a Informática); 2006-2007

[8] Bulnes F. Quantum intentionality and determination of realities in the spacetime through path integrals and their integral transforms. In: Bracken P, editor. Advances in Quantum Mechanics. Rijeka, Croatia: IntechOpen; 2013. DOI: 10.5772/53439. Available from: https:// www.intechopen.com/books/advancesin-quantum-mechanics/quantumintentionality-and-determination-ofrealities-in-the-space-time-throughpath-integrals-and-t 

Section 2

\section{Advanced Research in Hypercomplex Platforms in Quantum Communication}





\title{
A Novel Quantum Steganography Scheme Based on ASCII
}

\author{
Ri-Gui Zhou and Jia Luo
}

\begin{abstract}
Based on the novel enhanced quantum representation for quantum image (NEQR), a new blind quantum steganography scheme is proposed. In this scheme, an improved quantum representation of text utilizing ASCII is provided that uses two qubit sequences to store the same quantum text message. The general embedding process of the scheme is as follows: firstly, the cover image of sized $2^{n} \times 2^{n}$ will be divided into eight blocks of sized $2^{n-2} \times 2^{n-1}$, and the secret quantum text of sized $2^{n-2} \times 2^{n-1}$ is scrambled by Gray code transform method. Then, the disorder quantum text is embedded into the eight blocks of cover image employing the Gray code as a judgment condition. Meanwhile, the corresponding quantum circuits are drawn. Through the analysis of all quantum circuits, it can be concluded that the scheme has a lower complexity, that is, $\mathrm{O}(n)$. And the performance of the proposed scheme is analyzing in terms of simulation results of three items: visual quality, circuit complexity, and robustness.
\end{abstract}

Keywords: quantum steganography, quantum text, ASCII, Gray code, quantum circuit

\section{Introduction}

With the advantage of quantum physics, quantum computer has demonstrated a bright prospect over than the classic computer, especially in Grover's database searching algorithm [1] and Shor's prime factor decomposition algorithm [2].

Over the past few decades, teams of researchers have been noticed by quantum image processing that is a young emerging cross-discipline of image processing and quantum mechanics. The investigation in this direction is how to construct the quantum representations to represent images on quantum computer at first. So various quantum representations have been proposed, such as, Qubit Lattice [3], entangled image [4], real ket [5], flexible representation of quantum images (FRQI) [6], a novel enhanced quantum representation of digital images (NEQR) [7], multichannel representation for quantum images (MCRQI) [8], a normal arbitrary quantum superposition state (NAQSS) [9], and a novel quantum representation for color digital images (NCQI) [10]. Secondly, many kinds of quantum image

processing algorithms were developed, such as geometric transformations [11, 12], image translation [13-15], image scaling [16-18], image scrambling [19-21], image segmentation [22], feature extraction [23], edge detection [24], and image matching $[25,26]$. 
It is worth pointing out that the protection of network information, especially the increasing number of multimedia information on the network, has attracted researcher's attention. Thus information hiding was came into being a hot issue, which utilizes the sensory redundancy of the human sense organ to the digital signal, hiding a message in another ordinary message without changing the essential characteristics and use value of the ordinary message.

Similarly, quantum information hiding also includes steganography and watermarking, which have been gradually studied as the two main branches of quantum information hiding technology. In 2012, Iliyasu et al. proposed a quantum image watermarking algorithm based on restricted geometric transformations [27]. Zhang et al. introduced a protocol in 2013, that the watermark image was embedded into the Fourier coefficients of the quantum carrier image [28]. Later on, a dynamic watermarking scheme for quantum images based on Hadamard transform was proposed by Song et al. [29]. Two blind steganography algorithms based on LSB were proposed by Jiang et al. [30]. Miyake proposed a quantum watermarking scheme using simple and small-scale quantum circuits [31]. In this algorithm, the gray scale image was first used as a secret image. A watermarking scheme through Arnold scrambling and LSB was proposed by Zhou et al. [32], in which the quantum equal circuit was demonstrated. In 2017, Abd-El-Atty et al. proposed a new steganography scheme with Hadamard transformation [33]. In this scheme, the quantum text message was hided into the cover image. In addition, some algorithms that adopt color image as cover image have also been reported [34-37]. Wherein, a quantum copyright protection method based on a new quantum representation of text was presented by Heidari et al. [34].

In order to reduce the qubits of the representation of text in literature [34], we introduce an improved quantum representation of text. Then, the quantum text will be embedded in cover image through utilizing Gray code and quantum gates. Furthermore, the extracting procedure is absolutely blind and without any other help from classical computer.

The physical implementation of qubits and gates is difficult, for the same reasons that quantum phenomena are hard to observe in everyday life. One approach is to implement the quantum computers in superconductors, where the quantum effects become macroscopic, though at a price of extremely low operation temperatures.

In a superconductor, the basic charge carriers are pairs of electrons (known as Cooper pairs), rather than the single electrons in a normal conductor. At every point of a superconducting electronic circuit (that is a network of electrical elements), the condensate wave function describing the charge flow is well-defined by a specific complex probability amplitude. In a normal conductor electrical circuit, the same quantum description is true for individual charge carriers, however the various wave functions are averaged in the macroscopic analysis, making it impossible to observe quantum effects. The condensate wave function allows designing and measuring macroscopic quantum effects. And successive generations of IBM Q processors have demonstrated the potential of superconducting transmon qubits as the basis for electrically controlled solid-state quantum computers. But in this chapter, we focus on the theoretical design of quantum steganography scheme and describe it in the remaining sections.

The rest of the chapter is organized as follows. Section 2 gives fundamental knowledge of NEQR, Gray code and quantum equal circuit. The improved quantum representation of text is provided in Section 3. The proposed embedding and extracting procedures are depicted in Section 4. In Section 5, simulations and analysis that include visual quality, capacity, robustness, and computational complexity are provided. Finally, the conclusion is drawn in Section 6. 


\section{Preliminaries}

\subsection{NEQR}

For a gray scale image, a novel enhanced quantum representation of digital images (NEQR) was proposed in 2013 [7]. A quantum image can be described by the NEQR model as follows:

$$
|I\rangle=\frac{1}{2^{n}} \sum_{Y=0}^{2^{n}-1} \sum_{X=0}^{2^{n}-1}\left|C_{Y X}\right\rangle|Y\rangle|X\rangle=\frac{1}{2^{n}} \sum_{Y X=0}^{2^{2 n}-1} \otimes_{i=0}^{q-1} C_{Y X}^{i} \otimes|Y X\rangle
$$

where, $|Y X\rangle$ represents the position information and $\left|C_{Y X}^{i}\right\rangle$ encodes the color information.

$$
\begin{aligned}
& |Y X\rangle=|Y\rangle|X\rangle=\left|y_{n-1} y_{n-2} \ldots y_{0}\right\rangle\left|x_{n-1} x_{n-2} \ldots x_{0}\right\rangle, y_{i}, x_{i} \in\{0,1\}, i=0,1, \ldots, n-1 \\
& \left|C_{Y X}\right\rangle=\left|C_{Y X}^{q-1} C_{Y X}^{q-2} \ldots C_{y x}^{0}\right\rangle, C_{Y X}^{i} \in\{0,1\}, i=0,1, \ldots, q-1
\end{aligned}
$$

Thus, there are $q+2 n$ qubits being employed to store image information into a NEQR state for an $2^{n} \times 2^{n}$ image with gray range $\left[0,2^{q}\right]$. An example of an $2 \times 2$ image with ranged $\left[0,2^{8}-1\right]$, i.e., $n=2, q=8$ is demonstrated in Figure 1, in which the equation indicates that NEQR model stores the whole image in the superposition of the two entangled qubit sequences, encoding the color and position information, respectively.

Replace the entirety of this text with the main body of your chapter. The main body is where the author explains experiments, presents and interprets data of one's research. Authors are free to decide how the main body will be structured. However, you are required to have at least one heading. Please ensure that either British or American English is used consistently in your chapter.

\subsection{Gray code}

The typical binary Gray Code, called the Gray Code, was originally proposed by Frank Gray in 1953 for communication purposes and is now commonly used in analog-to-digital and position-to-digital conversion. In a group of Gray codes, there is only one different binary number between any two adjacent codes, as well as in the maximum and minimum numbers. By Gray code transform, the binary code can be converted into Gray code [21]. Denote $n(q)=n_{q-1} n_{q-2} \ldots n_{1} n_{0}$ as a $q$-bit binary code, where $n_{i}$ is a binary bit, the definition of Gray code transform is as follows:

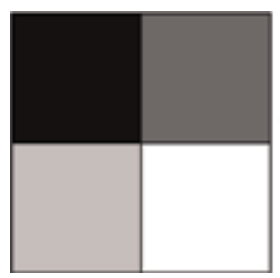

$$
\begin{aligned}
|I\rangle & =\frac{1}{2}(|0\rangle \otimes|00\rangle+|100\rangle \otimes|01\rangle+|200\rangle \otimes|10\rangle+|255\rangle \otimes|11\rangle) \\
& =\frac{1}{2}\left(\begin{array}{c}
|00000000\rangle \otimes|00\rangle+|01100100\rangle \otimes|01\rangle \\
+|11001000\rangle \otimes|10\rangle+|11111111\rangle \otimes|11\rangle
\end{array}\right)
\end{aligned}
$$

Figure 1.

A $2 \times 2$ example image and its representative expression in $N E Q R$. 

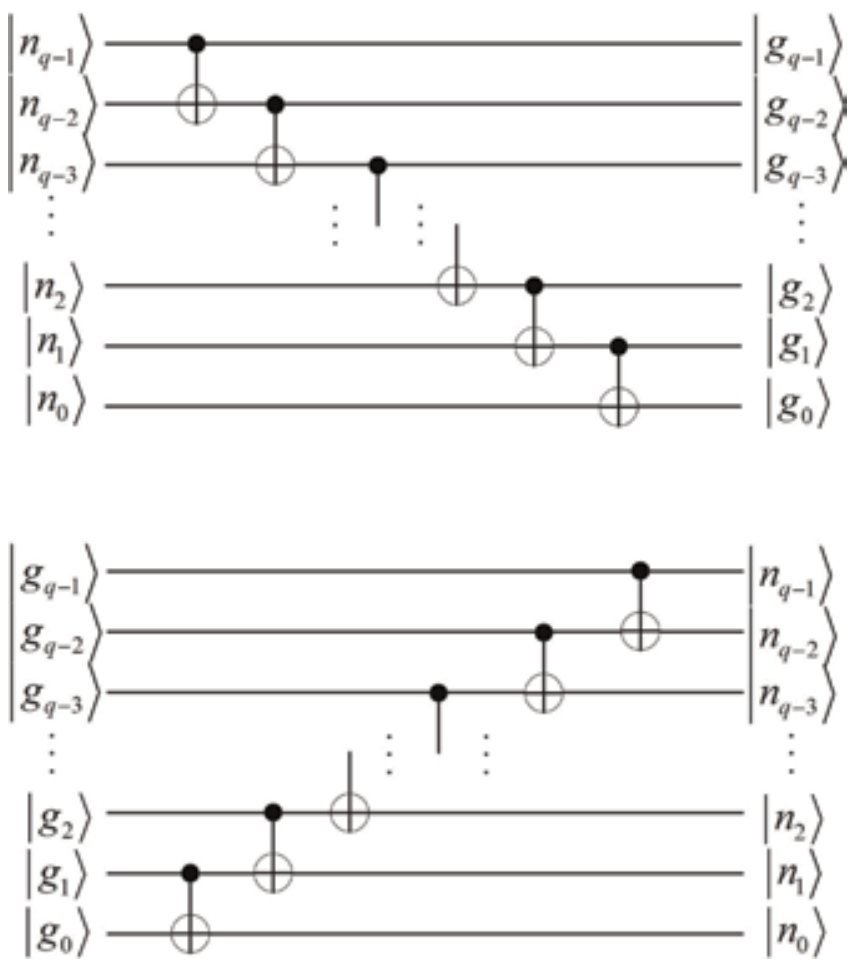

Figure 2.

Quantum circuits of (a) Gray code transform and (b) inverse Gray code transform.

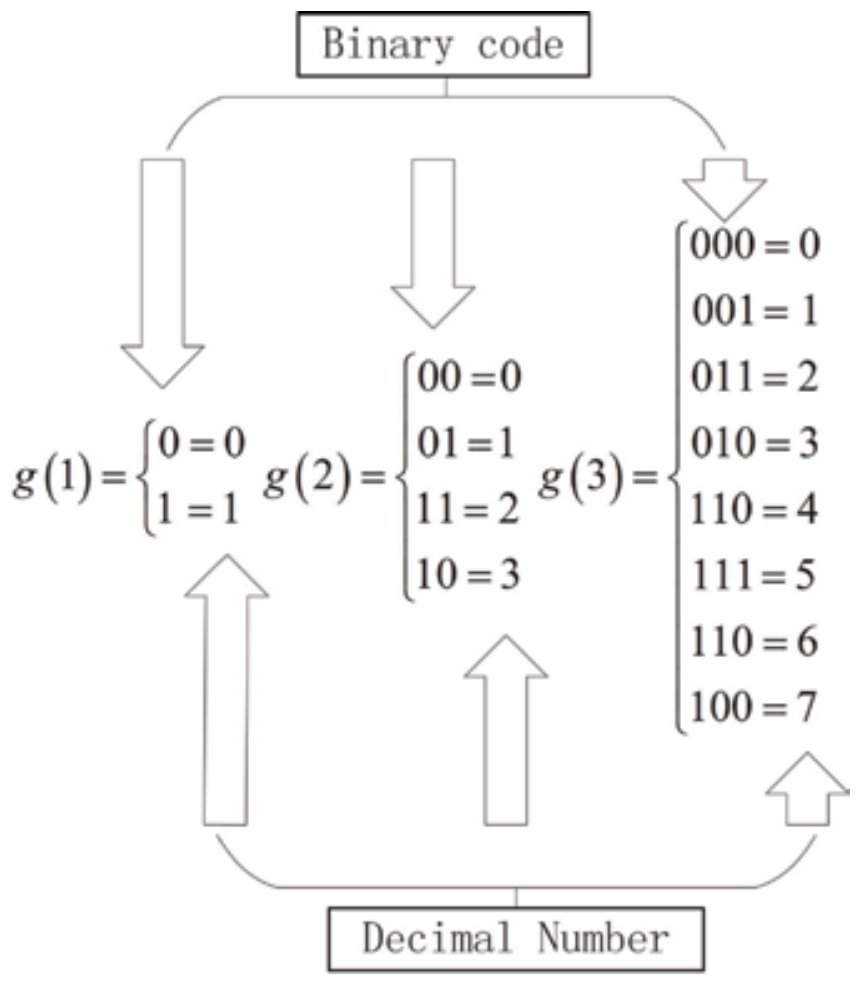

Figure 3.

1-bit, 2-bit and 3-bit Gray codes. 


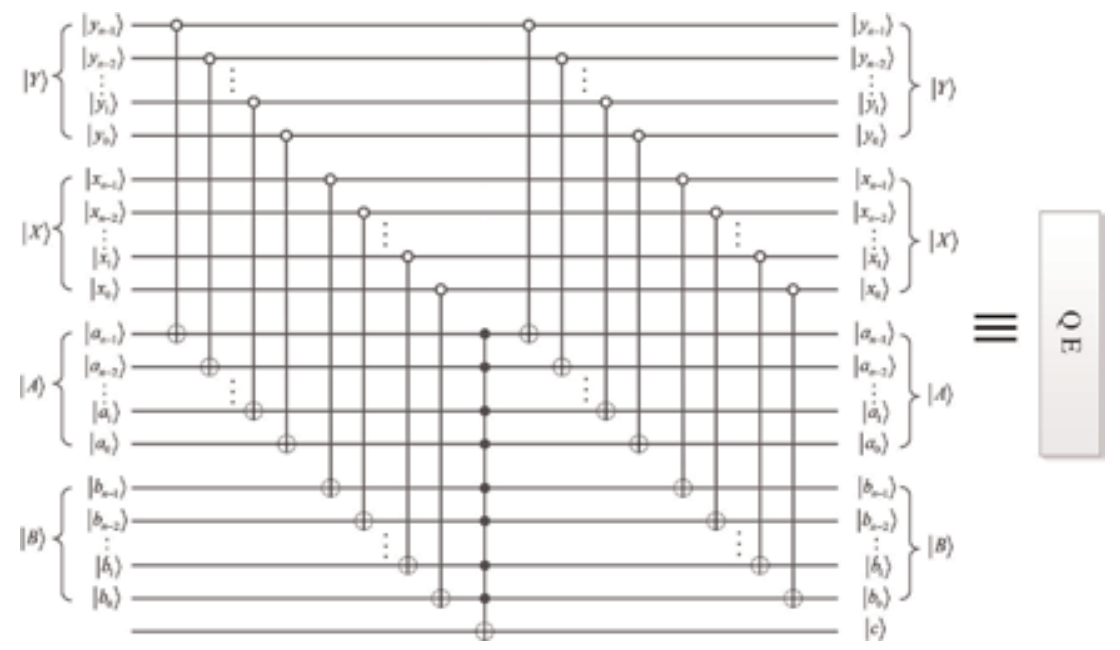

Figure 4 .

Quantum equal circuit.

$$
\begin{aligned}
& g_{q-1}=n_{q-1} \\
& g_{i}=n_{i} \oplus n_{i+1}, i=0,1, \ldots q-2
\end{aligned}
$$

and its inverse transform is:

$$
\begin{aligned}
& n_{i}=g_{i+1} \oplus g_{i}, i=0,1, \ldots q-2 \\
& n_{q-1}=g_{q-1}
\end{aligned}
$$

their corresponding quantum circuits are illustrated in Figure $\mathbf{2 a}$ and $\mathbf{b}$.

The transformed binary code $g(q)=g_{q-1} g_{q-2} \ldots g_{1} g_{0}$ is defined as the $q$-bit Gray code of $n(q)$. An example of Gray code where the bit number $q=1,2,3$ is shown in Figure 3.

\subsection{Quantum equal circuit}

In literature [32], Zhou et al. provided a quantum equal circuit to determine whether two qubit sequences are equal or not. The quantum circuit is shown in Figure 4, which compares $|Y X\rangle$ and $|A B\rangle$, where $|Y X\rangle=|Y\rangle|X\rangle=\left|y_{n-1} \ldots y_{0}\right\rangle\left|x_{n-1} \ldots x_{0}\right\rangle$ and $|A B\rangle=|A\rangle|B\rangle=\left|a_{n-1} \ldots a_{0}\right\rangle\left|b_{n-1} \ldots b_{0}\right\rangle, y_{i}, x_{i}, a_{i}, b_{i} \in 0,1, i=n-1, \ldots, 0$. Qubit $|c\rangle$ is output. If $|c\rangle=|1\rangle,|Y X\rangle=|A B\rangle$, otherwise, $|Y X\rangle \neq|A B\rangle$.

\section{The improved representation of quantum text}

ASCII (American Standard Code for Information Interchange) is a Latin alphabet-based computerized coding system that is the most versatile single-byte coding system available today [38]. The ASCII code uses the specified combination of 7-bit or 8-bit binary sequence to represent 128 or 256 possible characters. A standard ASCII code that uses 7-bit binary sequence (a total 8-bit sequence and the remaining 1-bit is 0) to represent all uppercase and lowercase letters, Arabic numerals, punctuation marks, and special control characters used in American 
English. Zero to thirty one and 127 (33 in total) are special characters for control or communication, and the rest are displayable characters. Figure 5 shows a table of characters that can be displayed.

Through analysis of the quantum text representation model proposed in literature [34], it is known that the model uses a seven-qubit sequence to store one character in the text message. In our proposed scheme, an improved quantum representation of text based on ASCII is proposed. Like NEQR model, the model including text message and position information. The text message $f(Y, X)$ on the corresponding coordinates $(Y, X)$ is encoded using ASCII of 8-bit binary sequence $T_{Y X}^{7} T_{Y X}^{6} \cdots T_{Y X}^{2} T_{Y X}^{1} T_{Y X}^{0}, T_{Y X}^{i} \in\{0,1\}, i=0,1, \ldots, 7$, this quantum textrepresentation model can be expressed as in Eq. (5) for a $2^{n} \times 2^{m}$ text.

\begin{tabular}{|c|c|c|c|c|c|}
\hline ASCII & symbol & ASCII & symbol & ASCII & symbol \\
\hline 00100000 & (space) & 01000000 & (a) & 01100000 & ' \\
\hline 00100001 & $!$ & 01000001 & $\mathrm{~A}$ & 01100001 & $\mathrm{a}$ \\
\hline 00100010 & $"$ & 01000010 & B & 01100010 & $\mathrm{~b}$ \\
\hline 00100011 & $\#$ & 01000011 & $\mathrm{C}$ & 01100011 & $\mathrm{c}$ \\
\hline 00100100 & $\$$ & 01000100 & D & 01100100 & d \\
\hline 00100101 & $\%$ & 01000101 & $\mathrm{E}$ & 01100101 & $\mathrm{e}$ \\
\hline 00100110 & $\&$ & 01000110 & $\mathrm{~F}$ & 01100110 & $\mathrm{f}$ \\
\hline 00100111 & $'$ & 01000111 & $\mathrm{G}$ & 01100111 & $\mathrm{~g}$ \\
\hline 00101000 & ( & 01001000 & $\mathrm{H}$ & 01101000 & $\mathrm{~h}$ \\
\hline 00101001 & ) & 01001001 & $\mathrm{I}$ & 01101001 & $\mathrm{i}$ \\
\hline 00101010 & * & 01001010 & $\mathrm{~J}$ & 01101010 & $\mathrm{j}$ \\
\hline 00101011 & + & 01001011 & $\mathrm{~K}$ & 01101011 & $\mathrm{k}$ \\
\hline 00101100 & , & 01001100 & $\mathrm{~L}$ & 01101100 & 1 \\
\hline 00101101 & - & 01001101 & $\mathrm{M}$ & 01101101 & $\mathrm{~m}$ \\
\hline 00101110 &. & 01001110 & $\mathrm{~N}$ & 01101110 & $\mathrm{n}$ \\
\hline 00101111 & 1 & 01001111 & $\mathrm{O}$ & 01101111 & 0 \\
\hline 00110000 & 0 & 01010000 & $\mathrm{P}$ & 01110000 & $\mathrm{p}$ \\
\hline 00110001 & 1 & 01010001 & $Q$ & 01110001 & q \\
\hline 00110010 & 2 & 01010010 & $\mathrm{R}$ & 01110010 & $r$ \\
\hline 00110011 & 3 & 01010011 & $\mathrm{~S}$ & 01110011 & $\mathrm{~s}$ \\
\hline 00110100 & 4 & 01010100 & $\mathrm{~T}$ & 01110100 & $\mathrm{t}$ \\
\hline 00110101 & 5 & 01010101 & $\mathrm{U}$ & 01110101 & $\mathrm{u}$ \\
\hline 00110110 & 6 & 01010110 & $\mathrm{~V}$ & 01110110 & $\mathrm{v}$ \\
\hline 00110111 & 7 & 01010111 & $\mathrm{~W}$ & 01110111 & $\mathrm{w}$ \\
\hline 00111000 & 8 & 01011000 & $\mathrm{X}$ & 01111000 & $\mathrm{x}$ \\
\hline 00111001 & 9 & 01011001 & $\mathrm{Y}$ & 01111001 & $\mathrm{y}$ \\
\hline 00111010 & $:$ & 01011010 & $\mathrm{Z}$ & 01111010 & $\mathrm{z}$ \\
\hline 00111011 & ; & 01011011 & {[} & 01111011 & \{ \\
\hline 00111100 & $<$ & 01011100 & 1 & 01111100 & $\perp$ \\
\hline 00111101 & $=$ & 01011101 & 1 & 01111101 & 3 \\
\hline 00111110 & $>$ & 01011110 & $\hat{\imath}$ & 01111110 & $\sim$ \\
\hline 00111111 & $?$ & 01011111 & & & \\
\hline
\end{tabular}

Figure 5 .

ASCII of displayable characters. 


$$
|T\rangle=\frac{1}{2^{n+m / 2}} \sum_{Y=0}^{2^{n}-1} \sum_{X=0}^{2^{m}-1}|f(Y, X)\rangle|Y\rangle|X\rangle=\frac{1}{2^{n+m / 2}} \sum_{Y X=0}^{2^{n+m}-1} \bigotimes_{i=0}^{7} T_{Y X}^{i} \otimes|Y X\rangle
$$

Figure 6 illustrates an example of a $2 \times 4$ text and its representative expression, where eight qubits are desired to store the text message and three qubits to store the position information. Therefore, this model just needs $8+n+m$ qubits to represent a $2^{n} \times 2^{m}$ text, namely there are $2^{n+m}$ symbols be stored. It is can be captured according to [34] which fifty-six qubits and $7 \times 2^{n+m}$ qubits are required to represent the text in this example and a text of $2^{n+m}$ symbols, respectively.

In order to embed the text information into quantum image, firstly, the text information needs to be transformed into a quantum state. The preparation procedure will now be described.

Step 1: this step is to prepare $8+n+m$ qubits that all are with state $|0\rangle$. The initial state $|\psi\rangle_{0}$ can be expressed as in Eq. (6):

$$
|\psi\rangle_{0}=|0\rangle^{\otimes n+m+8}
$$

Step 2: two single-qubit gates, $I$ and $H$ (shown in Eq. (7)), are used to transform $|\psi\rangle_{0}$ to the intermediate state $|\psi\rangle_{1}$, which is the superposition of all the characters of an empty text. The unitary operation $U_{1}$ can be written in Eq. (8):

$$
\begin{gathered}
I=\left[\begin{array}{ll}
1 & 0 \\
0 & 1
\end{array}\right], \quad H=1 / \sqrt{2}\left[\begin{array}{cc}
1 & 1 \\
1 & -1
\end{array}\right] \\
U_{1}=I^{\otimes 8} \otimes H^{\otimes(n+m)}
\end{gathered}
$$

The operation $U_{1}$ is setting on the $|\psi\rangle_{0}$ as shown in Eq. (9), and the position information is prepared in $|\psi\rangle_{1}$.

$$
\begin{aligned}
U_{1}\left(|\psi\rangle_{0}\right) & =(I|0\rangle)^{\otimes 8} \otimes(H|0\rangle)^{\otimes(n+m)} \\
& =\frac{1}{2^{n+m / 2}}|0\rangle^{\otimes 8} \otimes \sum_{i=0}^{2^{n+m}-1}|i\rangle \\
& =\frac{1}{2^{n+m / 2}} \sum_{Y=0}^{2^{n}-12^{m}-1}|0\rangle^{\otimes 8}|Y X\rangle \\
& =|\psi\rangle_{1}
\end{aligned}
$$

Step 3: In this step, $2^{n+m}$ sub-operations used to store the text message value for every position. In position $(Y, X)$, the unitary operation $U_{Y X}$ is shown below:

$$
\begin{gathered}
U_{Y X}=\left(I \otimes \sum_{j=0, i=0}^{2^{n-1}} \sum_{j i \neq Y X}^{2^{m-1}}|j i\rangle\langle j i|\right)+\Omega_{Y X} \otimes|Y X\rangle\langle Y X| \\
|T\rangle=\frac{1}{2^{3}}\left(\begin{array}{l}
|\mathrm{G}\rangle|000\rangle+|\mathrm{R}\rangle|001\rangle+|\mathrm{A}\rangle|010\rangle+|\mathrm{Y}\rangle|011\rangle \\
+|c\rangle|100\rangle+|0\rangle|101\rangle+|d\rangle|110\rangle+|e\rangle|111\rangle
\end{array}\right) \begin{array}{|l|l|l|l|}
\hline \mathrm{G} & \mathrm{R} & \mathrm{A} & \mathrm{Y} \\
\hline \mathrm{c} & \mathrm{o} & \mathrm{d} & \mathrm{e} \\
\hline
\end{array} \frac{1}{2^{3}}\left(\begin{array}{l}
|01000111\rangle|000\rangle+|01010010\rangle|001\rangle+|01000001\rangle|010\rangle+|01011001\rangle|011\rangle \\
+|01100011\rangle|100\rangle+|01101111\rangle|101\rangle+|01100100\rangle|110\rangle+|01100101\rangle|111\rangle
\end{array}\right)
\end{gathered}
$$

Figure 6.

A $2 \times 4$ text and its representative expression. 
where, $\Omega_{Y X}$ is a unitary operation as shown in Eq. (11), which is manipulating on $|\psi\rangle_{1}$ for altering digital representation of characters to the quantum state.

$$
\begin{aligned}
& \Omega_{Y X}=\stackrel{7}{i=0}_{Y X}^{i} \\
& \Omega_{Y X}^{i}:|0\rangle \rightarrow\left|0 \oplus T_{Y X}^{i}\right\rangle
\end{aligned}
$$

And if $T_{Y X}^{i}=1, \Omega_{Y X}^{i}$ is a $(n+m)$-CNOT gate, otherwise if $T_{Y X}^{i}=0$ then $\Omega_{Y X}^{i}$ is a quantum identity gate. Therefore, the text message value in position $(Y, X)$ is preparing by employing unitary operation $\Omega_{Y X}$ :

$$
\Omega_{Y X}|0\rangle^{\otimes 8}=\bigotimes_{i=0}^{7}\left(\Omega_{Y X}^{i}|0\rangle\right)=\bigotimes_{i=0}^{7}\left|0 \oplus T_{Y X}^{i}\right\rangle=\bigotimes_{i=0}^{7}\left|T_{Y X}^{i}\right\rangle=|f(Y, X)\rangle
$$

Applying $U_{Y X}$ on intermediate state $|\psi\rangle_{1}$, the transformation is shown in Eq. (13).

$$
\begin{aligned}
U_{Y X}\left(|\psi\rangle_{1}\right) & =U_{Y X}\left(\frac{1}{2^{n}} \sum_{j=0}^{2^{n-1}} \sum_{i=0}^{2^{m-1}}|0\rangle^{\otimes 8}|j i\rangle\right) \\
& =\frac{1}{2^{n}} U_{Y X}\left(\sum_{j=0, i=0}^{2^{n-1}} \sum_{j i \neq Y X}^{2^{m-1}}|0\rangle^{\otimes 8}|j i\rangle+|0\rangle^{\otimes 8}|Y X\rangle\right) \\
& =\frac{1}{2^{n}}\left(\sum_{j=0, i=0}^{2^{n-1}} \sum_{j i \neq Y X}^{2^{m-1}}|0\rangle^{\otimes 8}|j i\rangle+\Omega_{Y X}|0\rangle^{\otimes 8}|Y X\rangle\right) \\
& =\frac{1}{2^{n}}\left(\sum_{j=0, i=0}^{2^{n-1}} \sum_{j i \neq Y X}^{2^{m-1}}|0\rangle^{\otimes 8}|j i\rangle+|f(Y, X)\rangle|Y X\rangle\right)
\end{aligned}
$$

To store all the values to the quantum state, the whole operation $U$ that consists of $2^{n+m}$ sub-operations and shown in Eq. (14) is necessary. The final state $|\psi\rangle_{2}$ that is transformed from $|\psi\rangle_{1}$ is the improved representation of quantum text.

$$
\begin{aligned}
U & =\prod_{Y=0}^{2^{n}-12^{m}-1} \prod_{X=0}^{1} U_{Y X} \\
U\left(|\psi\rangle_{1}\right) & =U\left(\frac{1}{2^{n}} \sum_{j=0}^{2^{n-1} 2^{m-1}} \sum_{i=0}|0\rangle^{\otimes 8}|Y X\rangle\right) \\
& =\frac{1}{2^{n}} \sum_{j=0}^{2^{n-1}} \sum_{i=0}^{2^{m-1}} \Omega_{Y X}|0\rangle^{\otimes 8}|Y X\rangle \\
& =\frac{1}{2^{n}} \sum_{j=0}^{2^{n-1}} \sum_{i=0}^{2^{m-1}}|f(Y, X)\rangle|Y X\rangle=\left(|\psi\rangle_{2}\right)
\end{aligned}
$$

\section{Proposed scheme}

This section will discuss the particulars of embedding and extracting procedures about the proposed steganography scheme that hides a secret text into a cover grayscale image. Assume that the sizes for cover image and secret text are $2^{n} \times 2^{n}$ and $2^{n-2} \times 2^{n-1}$, respectively, the quantum representation can be formulated in Eqs. (15) and (16). 


$$
\begin{aligned}
& |C\rangle=\frac{1}{2^{n}} \sum_{Y X=0}^{2^{2 n}-1} \otimes_{i=0}^{7} C_{Y X}^{i} \otimes|Y X\rangle, \quad C_{Y X}^{i} \in\{0,1\} \\
& |T\rangle=\frac{1}{2^{2 n-3 / 2}} \sum_{Y X=0}^{2^{2 n-3}-1} \otimes_{i=0}^{7} T_{Y X}^{i} \otimes|Y X\rangle, \quad T_{Y X}^{i} \in\{0,1\}
\end{aligned}
$$

The general framework for the proposed scheme is shown in Figure 7, from which we can see that it is delineated into the classical and quantum domains. The preparation interface can transform classic image data into quantum states, which realizes the function of preparing the quantum image [7]. After the quantum image is stored in quantum states, our proposed quantum image steganography scheme can be implemented to transform the original quantum states to the desired states through the designed embedding circuits. Then, the quantum measurement operation is utilized to retrieve the processed image information. And once identified, the stego image is sent to the receiver by the public channel. The extraction operations are similarly for the receiver.

\subsection{Embedding procedure}

The embedding procedure in the proposed scheme is as follows.

1. Transform a classical cover image with $2^{n} \times 2^{n}$ size and eight bits gray scale into a quantum image $|C\rangle$ by NEQR, and transform a secret text with size $2^{n-2} \times 2^{n-1}$ into a quantum text $|T\rangle$ by ASCII expression.

2. Scramble the secret text $|T\rangle$ to be a meaningless text $|\hat{T}\rangle$ by Gray code transforming method.

3. The cover image will be divided into eight blocks of the same size and the secret text will be divided into eight bit-planes.

4. The divided eight bit-planes are embedded into eight blocks one by one.

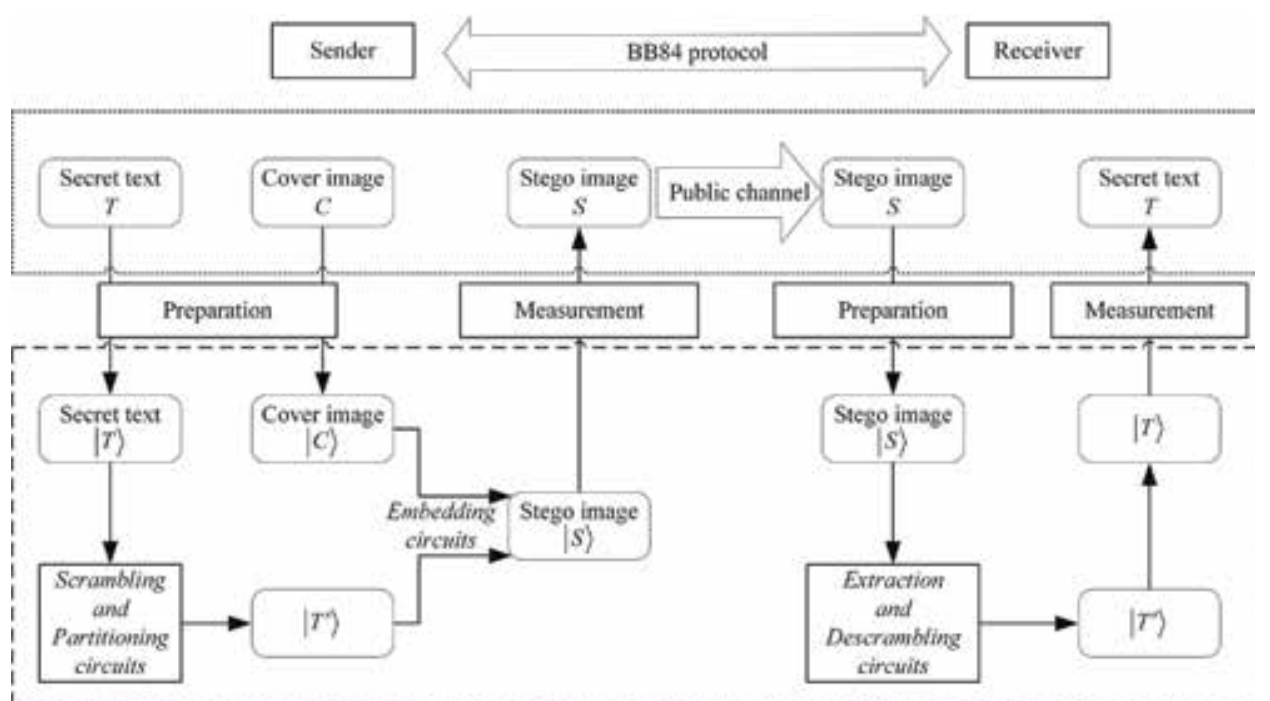

Figure 7.

The general framework of the proposed scheme. 


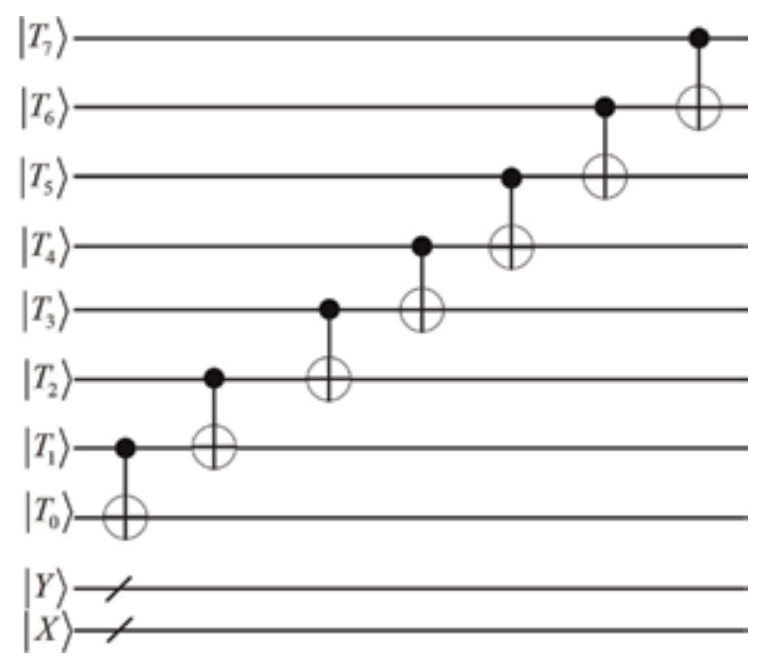

Figure 8.

Scrambling by Gray code transform.

\subsubsection{Scrambling}

For purpose of improving the security of secret text, the text message of $|T\rangle$ will be scrambled by Gray code transform before the embedding procedure. As mentioned in Subsection 2.2, in the eight qubits which store the text message, seven CNOT gates are used according to Gray code transform method, while the qubits representing for position information are not changed by quantum gates, the corresponding circuit is demonstrated in Figure 8.

\subsubsection{Partitioning}

In the proposed scheme, the $2^{n} \times 2^{n}$ cover image is divided into $4 \times 2$ blocks sized $2^{n-2} \times 2^{n-1}$. We define these blocks as $B_{i j}$, where $|i\rangle=\left|y_{n} y_{n-1}\right\rangle$ and $|j\rangle=\left|x_{n}\right\rangle$ are called control coordinates because if they are restricted as a specific value, then one of blocks will be selected. For example, if their values are equal to $|00\rangle$ and $|0\rangle$,

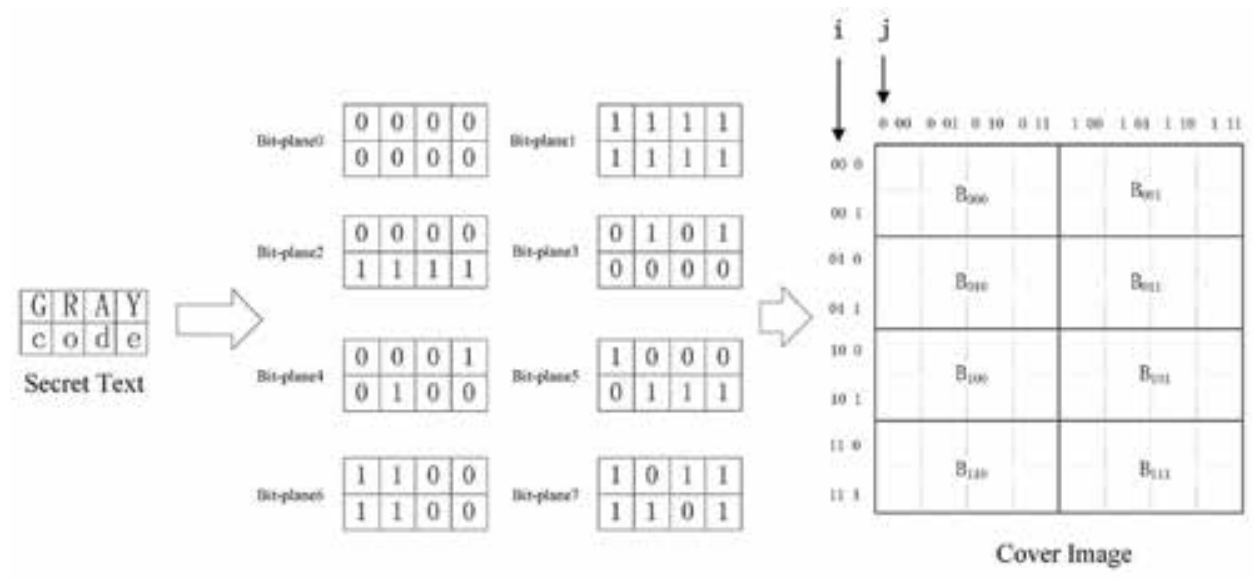

Figure 9.

An example of partition. 
then $2^{n-2} \times 2^{n-1}$ pixels in the top-left corner, that is, the first block $B_{000}$ is designated. For quantum text, the binary length of text message is eight, i.e., the text can be separated into eight bit-planes. Assume that the highest bit is embedded in $B_{000}$, the second highest bit is embedded in $B_{001}$, and so on. An example of partition with cover image size of $2^{3} \times 2^{3}$ and secret text size of $2^{1} \times 2^{2}$ is illustrated in Figure 9.

\subsubsection{Embedding}

After dividing the cover image, the quantum equal $(\mathrm{QE})$ circuit is used to compare the coordinates of a block and quantum text. Then, the stego image $|S\rangle$ is obtained after embedding process. More specifically, taking one of the blocks as an illustration, if the coordinates $\left|y_{n-3} y_{n-4} \ldots y_{0}\right\rangle\left|x_{n-2} x_{n-3} \ldots x_{0}\right\rangle$ of $|C\rangle$ is equal to the coordinates of $|\hat{T}\rangle,\left|\hat{T}^{i}\right\rangle$ is embedded in $\left|C^{0}\right\rangle$ by the following pseudo-code.

If $\left|\hat{T}^{i}\right\rangle=|1\rangle$

If $\operatorname{GRAY}\left(C_{Y X}^{7} C_{Y X}^{6} C_{Y X}^{5}\right)$ is even

$\operatorname{SWAP}\left(C_{Y X}^{0}, 1\right)$

Else if $G R A Y\left(C_{Y X}^{7} C_{Y X}^{6} C_{Y X}^{5}\right)$ is odd

$\operatorname{SWAP}\left(C_{Y X}^{0}, 0\right)$

End

If $\left|\hat{T}^{i}\right\rangle=|0\rangle$

If $\operatorname{GRAY}\left(C_{Y X}^{7} C_{Y X}^{6} C_{Y X}^{5}\right)$ is even

$\operatorname{SWAP}\left(C_{Y X}^{0}, 0\right)$

Else if $G R A Y\left(C_{Y X}^{7} C_{Y X}^{6} C_{Y X}^{5}\right)$ is odd

$\operatorname{SWAP}\left(C_{Y X}^{0}, 1\right)$

End

where the function $G R A Y(i)$ is the Gray code value of $i$, and the function $\operatorname{SWAP}(i, j)$ is to swap the value of $i$ and $j$. The corresponding embed block circuit is shown in Figure 10a and $\mathbf{b}$ presents the integrated embedding circuit that contains the selection of the block of cover image, the comparison of the coordinates, and the embedding process of the bit-planes of secret text into the LSB of cover image.

\subsection{Extraction procedure}

Like all the information hiding papers, only the receiver can extract the message. But it is worth pointing out the receiver only uses the stego image to extract the secret text in our scheme that means it is a blind scheme. The extracting procedure can be described as follows.

1. Extract and reorganize the bit plane from the stego image to obtain the disordered text $|\hat{T}\rangle$.

2. Descramble $|\hat{T}\rangle$ by using inverse Gray code transform in order to obtain original secret text $|T\rangle$. 


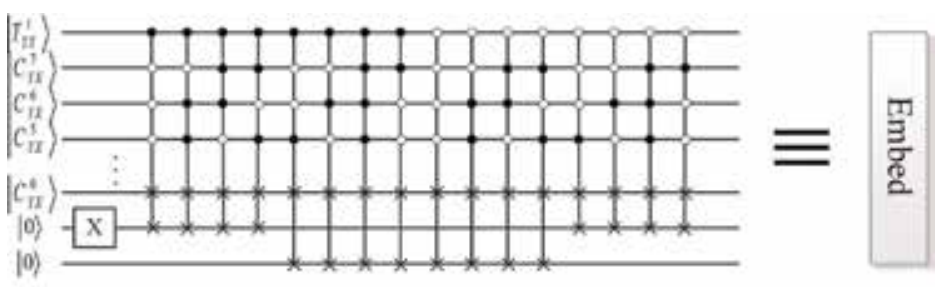

a

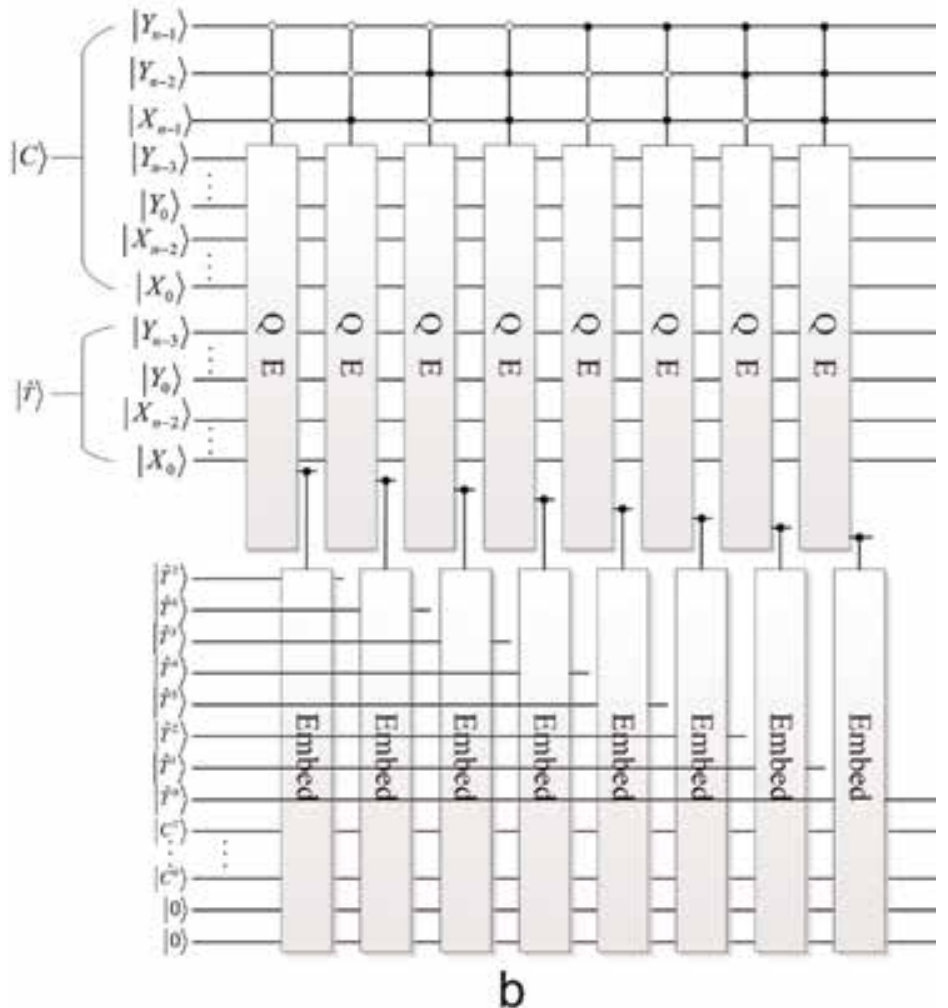

Figure 10.

Embedding circuit: (a) specific embedding block circuit and (b) the whole circuit.

\subsubsection{Extraction}

As can be seen from the above, the eight bit-planes of the secret text can be extracted from the eight blocks of the stego image. Accordingly, we can consider one block as an example. If the position of the stego image and the auxiliary blank quantum text are equal, and $\operatorname{GRAY}\left(S_{Y X}^{7} S_{Y X}^{6} S_{Y X}^{5}\right)$ is even and $C_{Y X}^{0}=1$ or $\operatorname{GRAY}\left(S_{Y X}^{7} S_{Y X}^{6} S_{Y X}^{5}\right)$ is odd and $C_{Y X}^{0}=0$, then $\left|\hat{T}^{i}\right\rangle=|1\rangle$. The corresponding circuit is shown in Figure 11a, and a whole extracting circuit that combines the extraction of all eight blocks together is exposed in Figure 11b.

\subsubsection{Descrambling}

Due to the operators used in embedding process are unitary, for this step of extracting process, we can use the inverse transpose of operators used in the embedding process. Figure 12 affords the quantum circuit to extract the secret image $|T\rangle$ from $|\hat{T}\rangle$. 
A Novel Quantum Steganography Scheme Based on ASCII DOI: http://dx.doi.org/10.5772/intechopen.86413
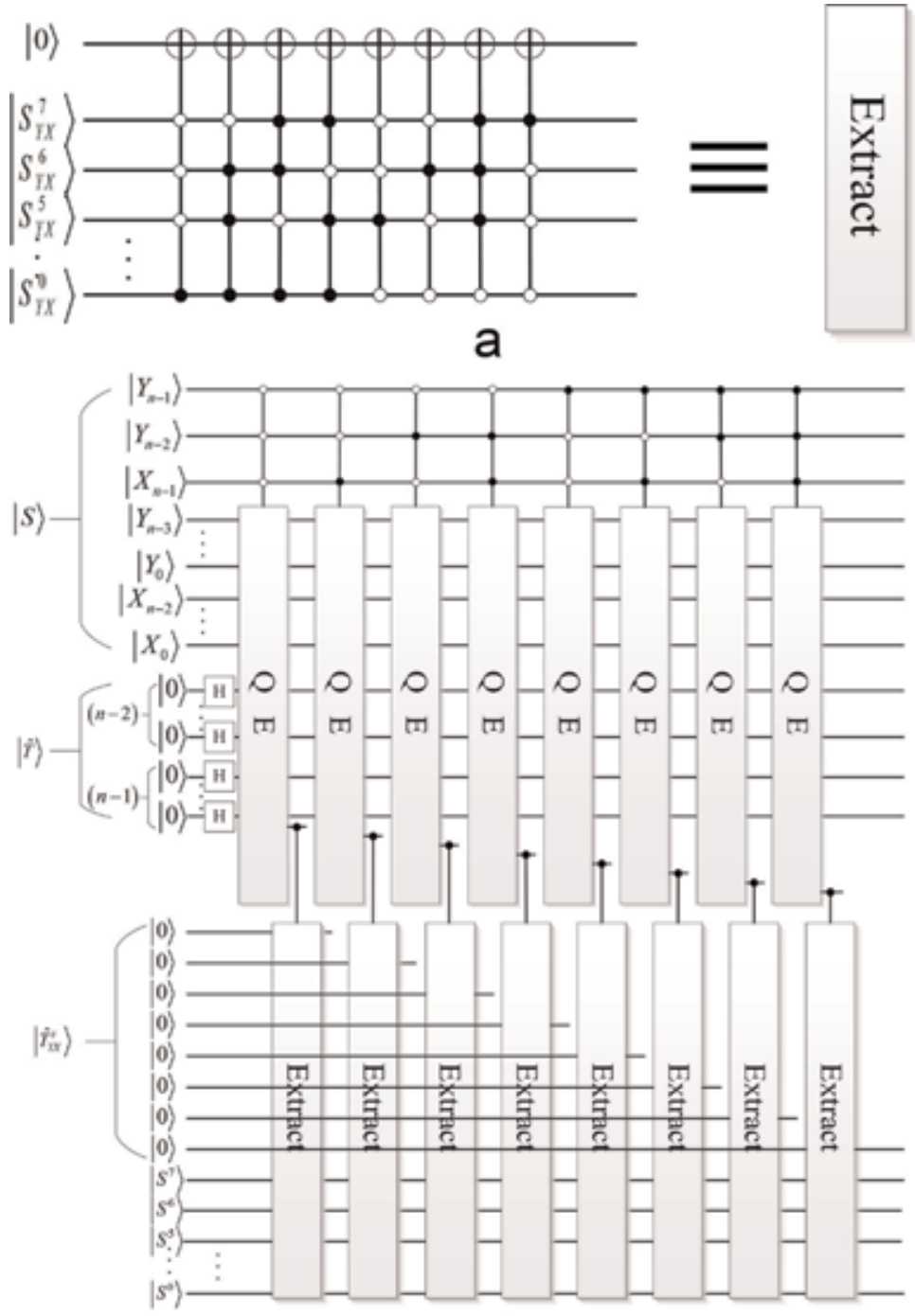

b

Figure 11.

Extracting circuit: (a) specific extracting block circuit and (b) the whole circuit.

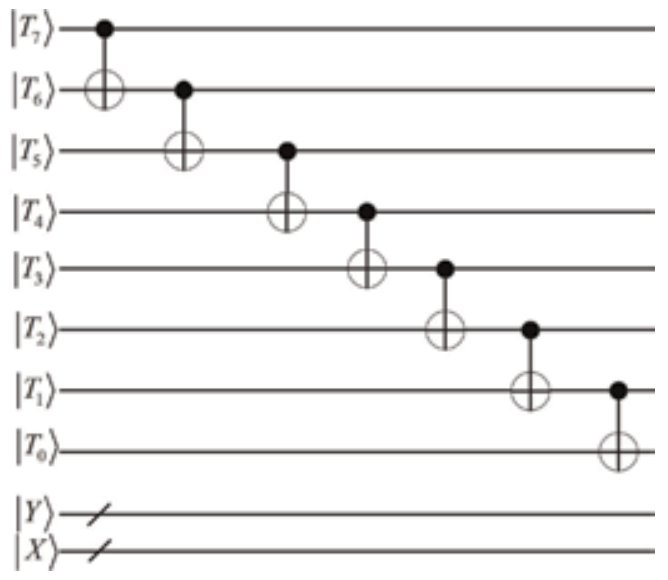

Figure 12.

Inverse transform. 


\section{Simulations and analysis}

In this section, some simulations and analysis of the results and properties of the proposed scheme are demonstrated. All the simulations are based on a classical computer equipped with software Matlab R2014b. The secret text of size $64 \times 128$ used in our proposed scheme is full of "Quantum Text and Quantum Image." And all the cover images are with size of $256 \times 256$, and shown in Figure 13 .

In general quantum image steganography scheme, the peak-signal-to-noise ratio (PSNR) is one of the most employed techniques to compare the fidelity of the stego image and the cover image. However, Iliyasu et al. explained that these available classical metrics are insufficient and/or ill-suited to effectively quantify the fidelity between two or more quantum images [39]. And in Refs. [39, 40], a wholly quantum-based metric to assess fidelity between quantum images (QIFM) is proposed. By using a statistical analysis they established that the proposed QIFM metric had a better correlation with digital image quality assessments of congruity than the other quantum image quality measures. And the formulation of the QIFM metric is the important in ensuring that applications sensitive to the peculiarities of quantum computation are formulated for effective quantum image processing (QIP). Before this, inspired to Ref. [41] that proposed a method to analyze the similarity between two quantum images of the same size based on the flexible representation of quantum images, a quantum image matching algorithm was introduced in [26], which sums up all the grayscale differences between two quantum images. In this chapter, we use the above two algorithms to assess the similarity of two images.

\subsection{Visual quality}

To verify the visual quality of the proposed scheme, we use the algorithm introduced in [26] to compare the congruity of the stego image and the cover image. Here, we briefly describe the algorithm as follows and the details can be acquired in [26].

Quantum stego image is directly mapped with quantum cover image, i.e., the quantum register representing each corresponding pixel of the quantum template image is subtracted from that of the quantum reference image by running a quantum subtractor. According to the quantum measurement results, all the grayscale difference can be summed. The smaller the sum, the higher the similarity between two quantum images, that is, the better visual quality of the stego image.

Table 1 shows the sum of all the grayscale differences between the stego image and the cover image, in which the secret text 1 is embedded.

Compared with the value 5,189,090 of Lena with watermark in Ref. [26], we can see that the values of the sum all the grayscale differences between cover image and stego image in our proposed scheme are smaller.
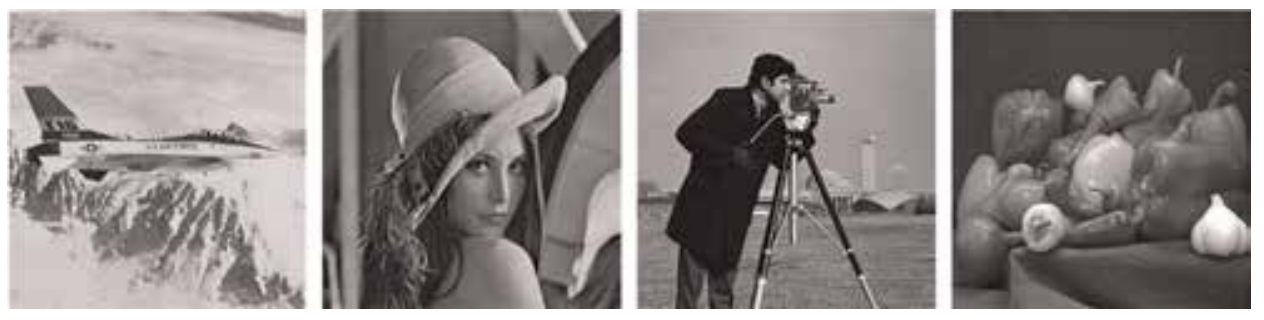

Figure 13.

Cover images used in the proposed scheme. 


\begin{tabular}{lcc}
\hline Cover image & Stego image & Sum of differences \\
\hline Lena & Stego-Lena & $4,026,525$ \\
\hline Airplane & Stego-airplane & $4,260,325$ \\
\hline Cameraman & Stego-cameraman & $4,455,338$ \\
\hline Pepper & Stego-pepper & $4,461,076$ \\
\hline
\end{tabular}

Table 1.

Sum of all the grayscale differences between cover image and stego image.

\subsection{Robustness}

In a noise-free environment, the proposed scheme can extract an intact secret text. However, the text extracting procedure is not always performed in a noiseless environment. The fidelity of the extracted text from the stego image under noise (simulate with salt and pepper noise) is verified, that is, using the QIFM metric. In this chapter, we give an outline of the steps of QIFM metric. For more details of the concepts and principles of QIFM, the reader can referred to [40].

Based on a pixel threshold (p) that assigns a value of zero or one to the pixel when $0 \leq p<127$ or $128 \leq p<255$, respectively. The content of both the cover and stego image is converted into their binary versions. Then the binary detail between cover image $I_{c}$ and stego image $I_{s}$ is evaluated using the following equation:

$$
\Gamma=I_{D c}-I_{D s}
$$

where the $I_{D(\text { c or s })}$ for an $N=n \times n$ pixel image is defined in:

$$
I_{D(\text { or } s)}=\left\{\begin{array}{l}
\frac{\sum\left(n_{(\text {or } s)}^{b}-n_{(\text {cor } s)}^{w}\right)}{N} ; \text { if } n_{(\text {or } s)}^{b} \neq n_{(\text {or } s)}^{w} . \\
\frac{\sum\left(n_{(\text {or } s)}^{b}-n_{(c \text { or } s)}^{w}\right)}{N}+1 ; \text { otherwise }
\end{array} .\right.
$$

Here, the notations $n_{c}^{b}$ and $n_{c}^{w}$ for the cover image and $n_{s}^{b}$ and $n_{s}^{w}$ for the stego image, which are referred to the number of white (0) and black (1) pixels in the cover and stego images.

Next, we count the number of pixel correspondences, $D$, which is defined as the number of pixels in cover image corresponding with pixels in stego image. And then, the total pixel-wise variation $B$ is computed by the equation:

$$
B=\frac{\sum B E R}{8 N}
$$

where BER denotes the bit error rate.

Finally, the fidelity of two images expressed in the form of a percentage is quantified by equation:

$$
F=\frac{D+(1-B) \times \Gamma}{N} \times 100
$$

As can be found from Table 2, four frequently-used cover images are used here as examples, when the value of the noise densities is set to 0.1 . The average value of 


\begin{tabular}{lc}
\hline Stego image & QIFM values of two texts \\
\hline Stego-Lena & 91.0899 \\
\hline Stego-airplane & 91.5049 \\
\hline Stego-cameraman & 91.6392 \\
\hline Stego-pepper & 91.1998 \\
\hline
\end{tabular}

Table 2.

QIFM values of the extracted text and their original secret text.

QIFM values is around 91, which is considered that the extracted text have a good fidelity.

\subsection{Circuit complexity}

The circuit complexity depends on the number of the elementary quantum gates. Thus, we take C-NOT gate as the basic unit. For our proposed scheme, the circuit complexity consists of two parts: embedding and extracting. In embedding part, the scrambling circuit complexity is 7. The embedding circuit is composed of eight QE circuits with three control qubits and eight embedding blocks with one control qubit. As the literature [42] pointed out, only $(4 k-8) 2-C-N O T$ gates are needed to construct one $k-C-N O T$ gate. Again, one SWAP gate is equivalent to three $C-N O T$ gates. The complexity of embedding circuit is:

$$
\begin{aligned}
& 8 \times[4 n \times(4 \times 4-8)+(4 \times 2 n-8)]+8 \times[16 \times 3 \times(4 \times 5-8)] \\
& =320 n-64+4608=320 n+4544
\end{aligned}
$$

In extracting part, the complexity of descrambling circuit is 7 , and the extracting circuit is consist of eight QE circuits with three control qubits and eight extracting blocks with one control qubit. The complexity of extracting circuit is:

$$
\begin{aligned}
& 8 \times[4 n \times(4 \times 4-8)+(4 \times 2 n-8)]+8 \times[8 \times(4 \times 5-8)] \\
& =320 n-64+768=320 n+704
\end{aligned}
$$

Therefore, the circuit complexity of the proposed scheme is $(640 n+5262)$, i.e., $\mathrm{O}(n)$.

\section{Conclusion}

This chapter proposes a new grayscale image steganography scheme which using NEQR representation to represent a $2^{n} \times 2^{n}$ cover image and presenting an improved representation of quantum text to store secret text with $2^{n-1+n-2}$ symbols. The Gray code of the highest three qubits of the gray value of the cover image is used as a judgment condition in embedded procedure. The acquisition of secret text is a process of extracting and reorganizing and inversely scrambling eight bit-planes, and it is worth mentioning that the process is absolutely blind. In addition, simulation results about the visibility quality and robustness of the proposed scheme are provided. And the circuit complexity is analyzed at last. 


\section{Acknowledgements}

This work is supported by the National Key R\&D Plan under Grant No. 2018YFC1200200 and 2018YFC1200205, National Natural Science Foundation of China under Grant No. 61463016 and "Science and technology innovation action plan" of Shanghai in 2017 under Grant No. 17510740300.

\section{Conflict of interest}

The authors declare no conflict of interest.

\section{Author details}

Ri-Gui Zhou ${ }^{1,2}$ and Jia Luo ${ }^{1,2 *}$

1 College of Information Engineering, Shanghai Maritime University, Shanghai, China

2 The Research Center of Intelligent Information Processing and Quantum Intelligent Computing, Shanghai, China

*Address all correspondence to: luojia@stu.shmtu.edu.cn

\section{IntechOpen}

(C) 2019 The Author(s). Licensee IntechOpen. This chapter is distributed under the terms of the Creative Commons Attribution License (http://creativecommons.org/licenses/ by/3.0), which permits unrestricted use, distribution, and reproduction in any medium, provided the original work is properly cited. (c) BY 


\section{References}

[1] Grover LK, Labs B, Avenue M, Nj $\mathrm{MH}$. A fast quantum mechanical algorithm for database search. In: Twenty-Eighth ACM Symposium on Theory of Computing. 1996. pp. 212-219

[2] Peter W, Labs AB, Ave M, Hill M. Algorithms for quantum computation: Discrete logarithms and factoring. In: Proceedings of 35th Annual Symposium on Foundations of Computer Scienece. 1994. pp. 124-134

[3] Venegas-Andraca SE, Bose S. Storing, processing and retrieving an image using quantum mechanics. In: Proceedings of SPIE_-The International Society for Optical Engineering. 2003

[4] Venegas-Andraca SE, Ball JL. Processing images in entangled quantum systems. Quantum Information Processing. 2010;9(1):1-11

[5] Latorre JI. Image compression and entanglement. Quantum Physics. 2005; 3:3-6

[6] Le PQ, Dong F, Hirota K. A flexible representation of quantum images for polynomial preparation, image compression, and processing operations. Quantum Information Processing. 2011; 10(1):63-84

[7] Zhang Y, Lu K, Gao Y, Wang M. NEQR: A novel enhanced quantum representation of digital images. Quantum Information Processing. 2013; 12(8):2833-2860

[8] Sun B, Le PQ, Iliyasu AM, Yan F, Garcia JA, Dong F, et al. A multichannel representation for images on quantum computers using the RGB D color space. In: 2011 IEEE International Symposium on Intelligent Signal Processing (WISP). 2011

[9] Qingxin HL, Zhou ZR, Yang LSX. Multi-dimensional color image storage and retrieval for a normal arbitrary quantum superposition state. Quantum Information Processing. 2014;13: 991-1011

[10] Sang J, Wang S, Li Q. A novel quantum representation of color digital images. Quantum Information Processing. 2017;16(2):42

[11] Le PQ, Iliyasu AM, Dong F, Hirota K. Fast geometric transformations on quantum images. International Journal of Applied Mathematics. 2010;40(3): 113-123

[12] Fan P, Zhou R, Jing N, Li H. Geometric transformations of multidimensional color images based on NASS. Information Sciences. 2016; 340-341:191-208

[13] Fan P, Zhou R. Quantum Gray-scale image translation transform the representation of quantum Gray-scale image. Quantum Information Processing. 2015;23:8763-8770

[14] Zhou R, Tan C, Ian H. Global and local translation designs of quantum image based FRQI. International Journal of Theoretical Physics. 2017;56(4): 1382-1398

[15] Wang J, Jiang N, Wang L. Quantum image translation. Quantum Information Processing. 2015;14: 1589-1604

[16] Zhou R, Hu W, Fan P, Ian H. Quantum realization of the bilinear interpolation method for NEQR. Scientific Reports. 2017;7(1): 2511

[17] Sang J, Wang S, Niu X. Quantum realization of the nearest-neighbor interpolation method for FRQI and NEQR. Quantum Information Processing. 2016;15(1):37-64 
[18] Jiang N, Wang L. Quantum image scaling using nearest neighbor interpolation. Quantum Information Processing. 2015;14(5):1559-1571

[19] Heidari S, Vafaei M, Houshmand M, Tabatabaey-Mashadi N. A dual quantum image scrambling method. Quantum Information Processing. 2019;2:1-23

[20] Jiang N, Luo WW. The quantum realization of Arnold and Fibonacci image scrambling. 2014;13:1223-1236

[21] Sun RZY, Fan P. Quantum image Gray-code and bit-plane scrambling. Quantum Information Processing. 2015; 14:1717-1734

[22] Caraiman S, Manta VI. Image segmentation on a quantum computer. Quantum Information Processing. 2015; 14(5):1693-1715

[23] Zhang Y, Lu K, Xu K, Gao Y, Wilson $R$. Local feature point extraction for quantum images. Quantum Information Processing. 2015;14:1573-1588

[24] Yi Z, Kai LU, Yinghui GAO. QSobel: A novel quantum image edge extraction algorithm. Science China Information Sciences. 2015;58(1):1-13

[25] Zhou RG, Liu XA, Zhu C, Wei L, Zhang X, Ian H. Similarity analysis between quantum images. Quantum Information Processing. 2018;17(6):1-12

[26] Yang YG, Zhao QQ, Sun SJ. Novel quantum gray-scale image matching. Optik. 2015;126(22):3340-3343

[27] Iliyasu AM, Le PQ, Dong F, Hirota $\mathrm{K}$. Watermarking and authentication of quantum images based on restricted geometric transformations. Information Sciences. 2012;186(1):126-149

[28] Zhang W-W, Gao F, Liu B, Wen Q$\mathrm{Y}$, Chen $\mathrm{H}$. A watermark strategy for quantum images based on quantum fourier transform. Quantum
Information Processing. 2013;12(2): 793-803

[29] Song X, Wang S, Abd AA. Dynamic watermarking scheme for quantum images based on Hadamard transform. Multimedia Systems. 2014;20(4): 379-388

[30] Jiang N, Zhao N, Wang L. LSB based quantum image steganography algorithm. International Journal of Theoretical Physics. 2016;55:107-123

[31] Miyake S, Nakamae K. A quantum watermarking scheme using simple and small-scale quantum circuits. Quantum Information Processing. 2016;15(5): 1849-1864

[32] Wenwen RZ, Ping H. Quantum watermarking scheme through Arnold scrambling and LSB steganography. Quantum Information Processing. 2017; 16(9):212-242

[33] Sang J, Wang S, Li Q. Least significant qubit algorithm for quantum images. Quantum Information Processing. 2016;15(11):4441-4460

[34] Heidari S, Gheibi R, Houshmand M, Nagata KA. Robust blind quantum copyright protection method for colored images based on owner's signature. International Journal of Theoretical Physics. 2017;56(8):2562-2578

[35] Li P, Zhao Y, Xiao H, Cao M. An improved quantum watermarking scheme using small-scale quantum circuits and color scrambling. Quantum Information Processing. 2017;16(5):127

[36] Qu Z, He H, Li T, Liu K, Li J, Zhu J, et al. Novel quantum watermarking algorithm based on improved least significant qubit modification for quantum audio. Chinese Physics B. 2018;27(1):010306

[37] Heidari S, Farzadnia E. A novel quantum LSB-based steganography 
method using the Gray code for colored quantum images. Quantum Information Processing. 2017;16(10):1-28

[38] Gorn S. Proposed revised american standard code for information interchange. Communications of the ACM. 1965;8(4):207-214

[39] Iliyasu AM, Abuhasel KA. A quantum-based image fidelity metric. In: 2015 Science and Information Conference (SAI); IEEE. 2015. pp. 664-671

[40] Iliyasu AM, Yan F. Metric for estimating congruity between quantum images. Entropy. 2016;18(10):360

[41] Yan F, Le PQ, Iliyasu AM, Sun B, Garcia JA, Dong F, et al. Assessing the similarity of quantum images based on probability measurements. In: 2012 IEEE Congress on Evolutionary Computation. 2012. pp. 10-15

[42] Barenco A, Bennett CH, Cleve R, Divincenzo DP, Margolus N, Shor P, et al. Elementary gates for quantum computation. Physical Review A. 1995; 52(5):3457-3467 


\title{
Quantum Algorithms for Fluid Simulations
}

\author{
René Steijl
}

\begin{abstract}
This chapter describes results of a recent investigation aiming to assess the potential of quantum computing and suitably designed algorithms for future computational fluid dynamics applications. For quantum computers becoming available in the near future, it can be expected that applications of quantum computing follow the quantum coprocessor model, where selected parts of the computational task for which efficient quantum algorithms exist are executed on the quantum hardware. For example, in computational fluid dynamics algorithm, this hybrid quantum/classical approach is discussed, and in particular it is shown how the approximate quantum Fourier transform (AQFT) can be used in the Poisson solvers of the considered method for the incompressible-flow Navier-Stokes equations. The analysis shows that despite the inevitable errors introduced by applying AQFT, the method produces meaningful results for three-dimensional example problems. A second example of a quantum algorithm for flow simulations is then described. This method based on kinetic modeling of the flow was developed to reduce the information transfer between quantum and classical hardware in the quantum coprocessor model. It is shown that this quantum algorithm can be executed fully on quantum hardware during a simulation. The conclusion summarizes further challenges for algorithm developments and future work.
\end{abstract}

Keywords: quantum algorithms, computational fluid dynamics, rarefied flows, kinetic modeling

\section{Introduction}

In recent years, the field of quantum computing [1] has developed into an active and diverse field of research, and significant progress has been made in a number of important areas. For a relatively small number of applications, quantum algorithms have been developed that provide a significant speedup relative to classical methods. Shor's algorithm for factoring composite integers and Grover's algorithm for quantum search were key developments in establishing quantum computing. More recently, significant progress has been made in the area of quantum chemistry and quantum physics. Beyond those two fields, only recently have quantum computing applications appeared in other areas of science and engineering, e.g., work in computational electromagnetics [2, 3], mixing in turbulent flow [4], and computational fluid dynamics [5]. More general applications have been developed which take advantage of the unique capabilities of quantum computing platforms, e.g., methods for the solution of linear systems of equations [6] and Poisson equation [7]. 
In recent years significant progress has been made in designing and constructing quantum computers. Currently available quantum computers are relatively small-scale and have become known as noisy intermediate-scale quantum (NISQ) computers. These machines have a limited number of qubits (expected to increase to 50-100 in coming years), a limited connectivity between these qubits, a small set of available quantum gates, and typically very little or no quantum error correction.

This chapter describes results of a recent investigation aiming to assess the potential of quantum computing and suitably designed algorithms for future computational fluid dynamics application, particularly for NISQ-type quantum hardware. In this work, the quantum circuit model is used for a "universal" or "digital" quantum computer, i.e., work on adiabatic quantum computing is not considered here. In the absence of the required quantum hardware, large-scale parallel simulations on parallel classical computers are required in developing such algorithms. In this work the recently developed quantum simulator [5] included in the MФC multi-physics CFD framework is used [8,9].

In the near future, the most likely scenario for the introduction of quantum computing hardware is through the quantum coprocessor model, i.e., where a quantum processing unit (QPU) is loosely coupled to a classical computer with one or more CPUs [10]. In current designs, the quantum processor requires storage at low temperatures in a cryostat leading to a distinct physical separation between the classical and quantum hardware. Coupling takes place by exchanging classical information. In application of this hybrid quantum/classical approach, the quantum processor acts like a coprocessor with the quantum processor dealing with selected computationally demanding tasks. The quantum processor receives information from the CPU, and this is used to initialize the quantum state in the quantum processor. During the quantum simulation, the quantum state is transformed by application of quantum gates in quantum circuits. Then measurement operations are used to extract classical information from this quantum state, and this is subsequently passed to the CPU. Since in quantum mechanics a measurement leads to the (partial) collapse of the quantum state, in the hybrid classical/quantum approach, typically multiple realizations of the quantum state are needed to obtain classical information with acceptable levels of noise and uncertainty. It is important to recognize that, since initializing a particular quantum state in quantum computer can be a significant challenge, this hybrid approach can only be expected to lead to significant computational speedups in case the quantum simulation is significantly faster for the selected problem than conventional solution methods.

As an example of this hybrid classical/quantum approach, the author introduced a quantum computing application in which the vortex-in-cell method was used to solve the incompressible-flow Navier-Stokes equations in a regular domain [5]. In this algorithm, the Poisson solvers dominating CPU time requirements are based on the quantum computing equivalent of the fast Fourier transform, i.e., the quantum Fourier transform. In this chapter, this algorithm and its application to example flow problems are investigated further. Specifically, the effect of applying an approximate QFT instead of the full QFT is analyzed for different levels of approximation or truncating of rotation gates in the quantum circuit implementation.

The second part of this chapter describes a more recent investigation into the development of quantum algorithms relevant for computational aerodynamics based on modeling at the kinetic level. The key innovation in these developments is the design targeting execution of the algorithm fully on the quantum processor. In particular, at the start of the simulation, multiple quantum states in the quantum processor would be initialized. Then, the quantum algorithms would perform a series of unitary transformations. Only at the end of the simulation would 
measurements be performed to extract the required classical. A key question this study aims to answer is for which applications this approach is feasible.

This chapter is organized as follows. Section 2 describes key principles of quantum computing relevant to the quantum algorithms for fluid simulations described here. Section 3 describes the hybrid quantum/classical implementation of the vortex-in-cell method along with a number of example applications. The quantum discrete-velocity algorithm for kinetic flow modeling is described in Section 4. Conclusions and future research directions are presented in Section 5.

\section{Elements of quantum computing relevant in current work}

The fundamental unit of quantum computation is the qubit [1]. Whereas a classical bit is confined to existing in either the 0 or 1 state, a qubit can be in a state of superposition, i.e., it exists in both states simultaneously. Upon measuring the qubit, the quantum state collapses to either of these two states, and the qubit is no longer in a state of superposition. The state of a qubit is defined through a pair of complex numbers [1]. A collection of $n q$ qubits in a coherent state is termed a quantum register of size $n q$ here. Its quantum state is defined by the wave function $\mid \Psi>$ resulting from the tensor product of the quantum states of each qubit in the coherent register. The superposition in this coherent register then creates $2^{\text {nq }}$ different states that can be found upon measurement of the quantum state. In simulating this quantum state on a classical computer, a storage space of $2^{\text {nq }}$ complex numbers is required.

In the present work, the quantum circuit model of quantum computing is used. In this case, the unitary operations on a quantum state allowed by quantum mechanics are represented by a series of quantum (logic) gates acting on the quantum state. A quantum logic gate is an elementary quantum computing device that performs a fixed unitary operation on selected qubits in a fixed period of time. Written in a matrix form, unitary means that the determinant of the transformation equals one.

\subsection{Mapping a computational problem onto the quantum state vector}

The quantum state $\mid \Psi>$ for a register with $n q$ coherent qubits is represented by a Hilbert space of dimension $2^{\text {nq }}$. In a quantum computer, different possibilities exist for the physical implementation of qubits, e.g., the "spin" of an electron (with the two possible states being "spin-up" and "spin-down") or the plane of polarization of linearly polarized photon has been used. The discrete energy levels in an atom excited by laser pulses present an alternative to electron-spin and photon-based qubit implementations.

We will now describe how this quantum state can be used to represent the storage space required for the computational problems of interest here, representing discretized partially differential equations. As a first step, consider a function $f$ discretized on a (regular) mesh with $N$ mesh points. It follows that $\log _{2} N$ qubits would suffice to create the required number of degrees of freedom in the quantum state vector.

However, it is important to stress that the quantum state vector only represents the likelihood that upon measurement the quantum state collapses into a particular state [1]. In other words, with $n q=\log _{2} N$, we cannot extract the full information for all $N$ degrees of freedom using a single realization of this quantum state. However, for as long as this classical information is not needed, the quantum state has the required number of degrees of freedom. This superposition-based principle for 


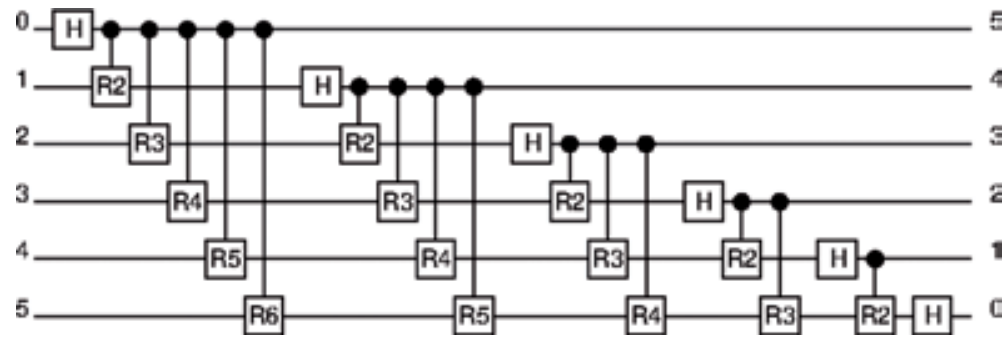

Figure 1.

Quantum circuit for QFT on six-qubit register.

storage of discrete data can be extended to multidimensional problems as well. For example, 24 qubits suffice to store a single discretized function on a $256^{3}$ regular mesh. In application in which multiple variables are to be stored in each mesh point, as in the discrete-velocity method discussed in the second part of this chapter, we add further qubits to the quantum register. Specifically, for each qubit added in the coherent register, the number of degrees of freedom is doubled. Once a mapping of the considered computational problem onto the quantum state vector has been designed, calculations are then performed through application of quantum gates as in the quantum circuit model.

\subsection{Approximate quantum Fourier transform (AQFT)}

The ability to implement the quantum Fourier transform (QFT) efficiently on a quantum computer is of paramount importance for many quantum algorithms. Figure 1 shows the "standard" quantum circuit implementation of the QFT for an example register with 6 qubits. In Figure 1, "H" represents the one-qubit Hadamard gate, and the "Rk" gates are controlled rotation gates over an angle defined by index "k," i.e., $2 \pi / 2^{k}$. In this circuit and all subsequent quantum circuits shown in this chapter, the qubit register is represented vertically, with the leftmost qubit in the register at the top. The horizontal direction determines the sequence of gates that are applied to the quantum state. In the QFT example shown, it can be seen that the qubit indices have been reversed in the output state (right-hand side) relative to the input, to represent that this standard QFT circuit returns the discrete Fourier transform in bit-reversed ordering.

A particular challenge is presented by the controlled rotation gates particularly those involving small angles. The QFT can be implemented approximately by removing all rotation gates with angles smaller than a certain threshold value, resulting in the approximate $\mathrm{QFT}$ (AQFT). In particular for fault-tolerant implementations, this is desirable as it greatly reduces the gate count. In the following, we define the approximation or "band-limiting" in the AQFT as follows. The rotation gates are eliminated above a limit value " $k$," i.e., for an angle smaller than $2 \pi / 2^{k}$, the rotation gate is not included in the quantum circuit.

\section{Hybrid quantum/classical vortex-in-cell method}

The vortex-in-cell method is a well-studied hybrid particle-mesh method for incompressible flows and is particularly well suited for flows in regular domains such that efficient Poisson solvers can be used. In the present work, the Fourier 
analysis approach to solving the problem in a fully periodic domain is used, using the QFT for the required discrete Fourier transforms.

The vortex-in-cell (VIC) method solves the incompressible-flow Navier-Stokes equations, transformed into the Helmholtz equations for vorticity evolution [5]:

$$
\frac{\partial \vec{\omega}}{\partial t}+\vec{u} \frac{\partial \vec{\omega}}{\partial \vec{x}}=(\vec{\omega} . \nabla) \vec{u}+\nu \Delta \vec{\omega} ; \vec{\omega}=\nabla x \vec{u}
$$

In simulations using the VIC, the flow evolves through a (large) number of time steps. During each of these time steps, the velocity field is recomputed using solutions of three Poisson problems for stream function $\vec{A}$ :

$$
\Delta \vec{A}=-\vec{\omega} ; \vec{u}=\nabla x \vec{A}
$$

This part of the VIC is of particular interest here, as it represents the part that would be performed by the quantum processor in the quantum coprocessor model.

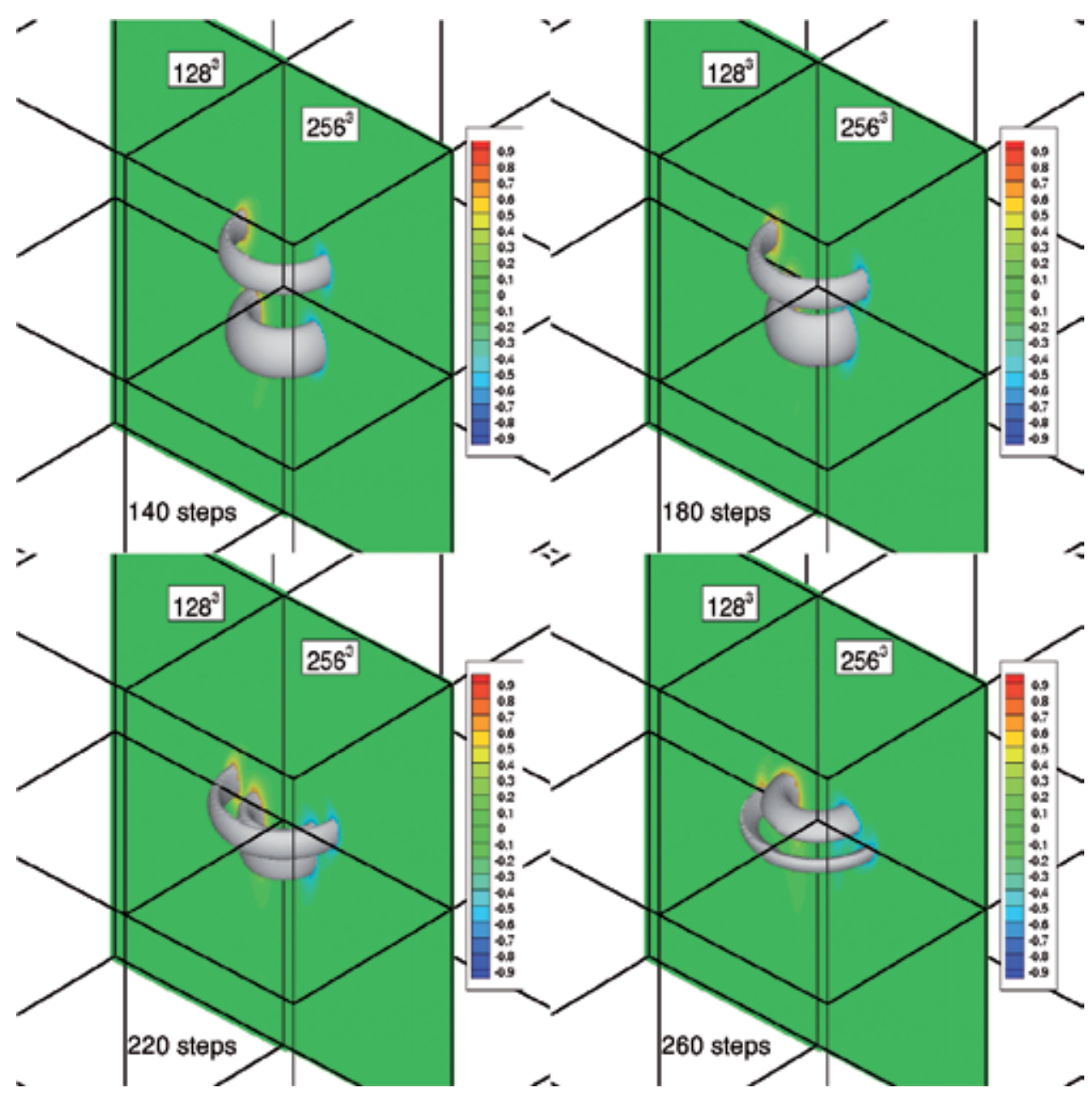

Figure 2.

Vortex-in-cell simulation of leapfrogging vortex rings. Effect of mesh refinement is shown. "Noiseless" simulation using full QFT. 


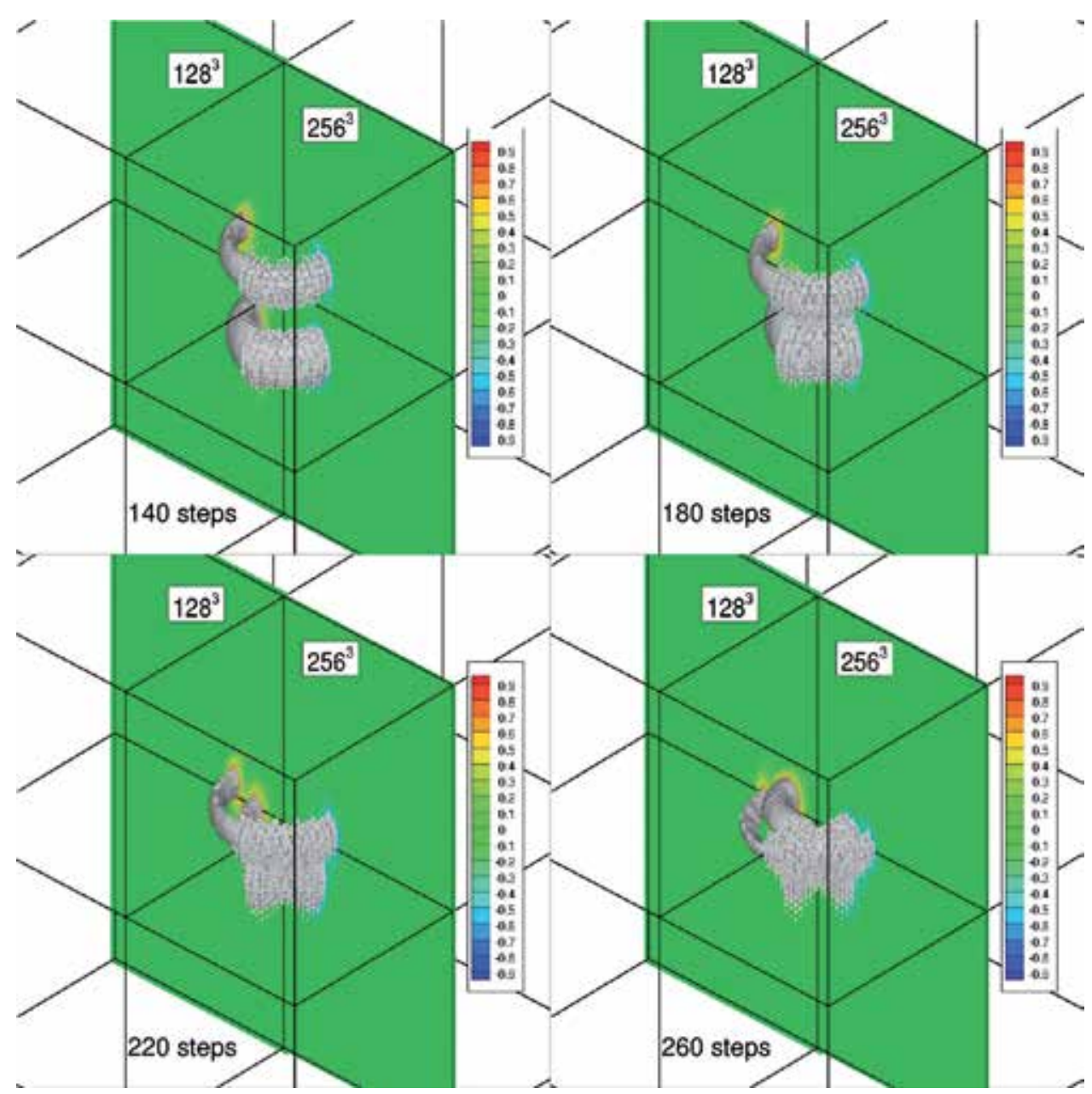

Figure 3.

Vortex-in-cell simulation of leapfrogging vortex rings. For two different mesh resolutions, the effect of applying AQFT is shown (" $k$ " limit is 5).

Figure 2 shows an example of a VIC simulation of two leapfrogging vortex rings, i.e., flow structures of fundamental importance in fluid mechanics. The lower vortex ring is stronger than the ring above it, and it will therefore convect upward faster, leading to the interaction of the vortex rings as shown. The iso-surface represents vorticity strength, i.e., a direct indicator of the "strength" of the considered vortex. Results are compared for two different meshes, $128^{3}$ and $256^{3}$, to highlight the dependency of the solution on the chosen mesh size. Also, in the shown simulation, no quantum errors were simulated, and the full QFT was used. If we now replace the QFT with the AQFT, the results shown in Figures $\mathbf{3}$ and $\mathbf{4}$ are obtained. In Figure 3, the "k" limit in the QFT is set to five for both meshes, showing that for the finer mesh this leads to unacceptable errors, while the coarser-mesh simulation still produces worthwhile results. If the " $k$ " limit for the finer mesh is increased from 5 to 6, i.e., more controlled rotation gates are included in the AQFT circuit, the simulation on the finer mesh can also be made to produce similarly useful results. These example results show what level of approximation in the QFT is tolerable for application of the VIC method. For other QFT-based CFD solvers, a similar sensitivity study would need to be conducted. 


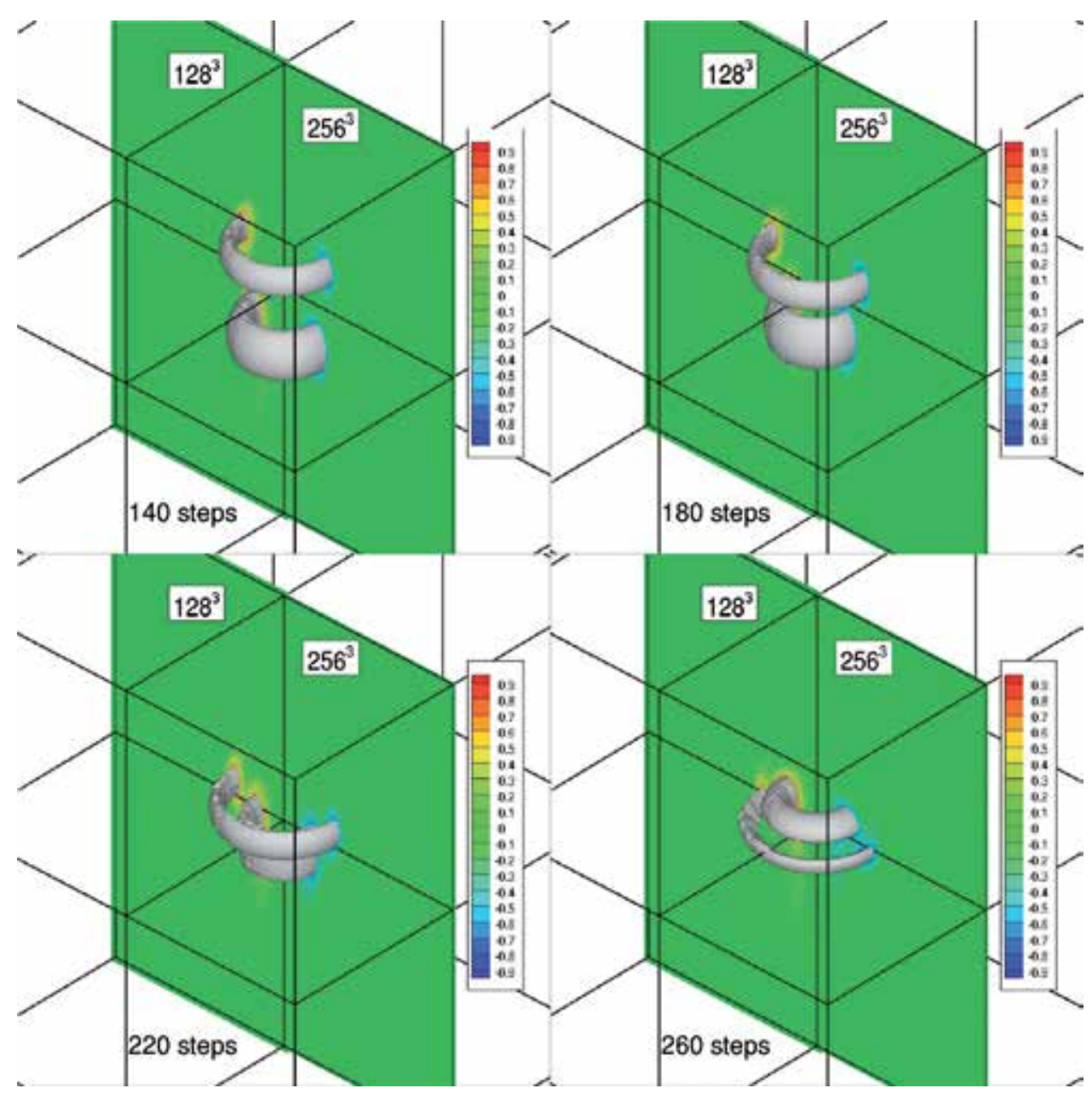

Figure 4.

Vortex-in-cell simulation of leapfrogging vortex rings. For 1283 mesh, AQFT (" $k$ " limit 5) is used. For 2563 mesh, "k" limit in AQFT is 6.

\section{Quantum algorithm for discrete-velocity method}

In computational fluid dynamics, the most widely used methods involve solving the Navier-Stokes equations for a continuum fluid, i.e., where fluid density, velocity components, and energy in each location in the computational domain are to be found from conservation equations. The vortex-in-cell method used in the previous section employs the Navier-Stokes equations in a transformed form involving vorticity rather than velocity, but importantly it still uses the continuum flow assumption.

An alternative approach to the Navier-Stokes-based modeling is a description of the flow at a more detailed level, i.e., at the kinetic level [11]. Instead of governing equations for mass, momentum, and energy conservation, the flow is now described by the Boltzmann equation governing a particle distribution function in state space (or 3D velocity space for a 3D monatomic gas flow) for each location in the considered domain [10].

For a monatomic gas, the distribution function $f(\vec{x}, \vec{c} ; t)$ with $\vec{x}$ defining the coordinates in (physical) space and $\vec{c}$ the molecular velocity (defined in "velocity space") is governed by the Boltzmann equation: 


$$
\frac{\partial \mathrm{f}}{\partial \mathrm{t}}+\overrightarrow{\mathrm{c}} \frac{\partial \mathrm{f}}{\partial \overrightarrow{\mathrm{x}}}=\mathrm{Q}(\mathrm{f}, \mathrm{f})
$$

The distribution function defines for each point $\vec{x}$ the likelihood that molecules have velocity $\vec{c}$. On the right-hand side of the equation, the collision term $Q(f, f)$ represents the effect of collisions between particles [12]. For each point in space, we can take moments of the distribution function to obtain the local fluid density, velocity components, and energy.

The key advantage of this approach is that non-continuum flows, i.e., flows for which the density is so low that we cannot assume it to act as a continuum, can also be modeled. However, the main problem is the large computational cost when using a direct discretization approach due to the high dimensionality, i.e., for a 3D flow problem, we have a six-dimensional solution space (or seven when including time).

A further main challenge is the cost of evaluating the collision term. For the free-molecular flows considered here, the collision term can be discarded, and we will use the collisionless Boltzmann equation instead.

In the discrete-velocity method used here, the velocity space is discretized using a uniformly spaced Cartesian mesh. A maximum molecular velocity magnitude is defined, $c_{\max }$, so that the left and right domain boundaries in velocity space are at $-c_{\max }$ and $+c_{\max }$, respectively, with a uniform spacing $\Delta \mathrm{c}$ separating each point in velocity space. These limits are problem dependent.

The discrete-velocity approach used here has a number of characteristics facilitating a quantum implementation:

1. A uniformly spaced Cartesian mesh is used for the spatial discretization as well as for the discretization of the velocity space.

2. In case solid objects are present in the computational domain, these are rectangular, and its edges align with the mesh lines in the mesh. Specifically, solid bodies can be defined by "tagging" selected groups of cells in the mesh.

3. A constant velocity-space discretization is used in each point in space, i.e., the velocity-space boundaries defined earlier as well as the number of discrete velocities are identical in each cell.

4. The convection part of the Boltzmann equation (i.e., the second term on the left-hand side in the shown equation) along with gas-solid interactions determines the time evolution of the distribution function in the absence of interparticle collisions.

5. The time-integration method used here is based on the reservoir technique [13], such that during the time integration the convection step always exactly involves the distribution function defined in a cell of computational mesh to move to a cell that is a nearest neighbor. This is commonly referred to as "streaming" of data.

In the examples shown, problems are restricted to two-dimensional flows. For this case, the collisionless Boltzmann equation originally defined for $3 \mathrm{D}$ can be reduced to two kinetic equations for two reduced distribution functions:

$$
\frac{\partial f}{\partial t}+\vec{c} \frac{\partial f}{\partial \vec{x}}=0 ; \frac{\partial g}{\partial t}+\vec{c} \frac{\partial g}{\partial \vec{x}}=0
$$


where the velocity and coordinate vectors are now defined in 2D.

Inspired by the previous work on the Dirac equation [14], an efficient quantum circuit implementation of the streaming operations in $\mathrm{x}$ - and $\mathrm{y}$-direction on a Cartesian 2D mesh can be created as shown in Figure 5. The example shows how a 12-qubit register is used for a discretized function with 6 qubits defining the indices of 64 grid points in $\mathrm{x}$ - and $\mathrm{y}$-coordinate directions. The circuits with the filled circles define streaming to "right" neighbors, i.e., when each control qubits has the |1> state, the target qubit gets negated ("X" symbol used here). Similarly, the left streaming operation employs multiple-control NOT gates with target qubits being negated when each control qubit is in state $\mid 0>$.

For the streaming operations in quantum discrete-velocity method, further extensions are needed. First, the quantum register needs to be extended relative to that shown in Figure 5 to account for the storage of two discretized reduced distributions defined in the discretized velocity space. The additional distribution function is accounted for using an additional qubit termed " $\mathrm{g}$ " in the following quantum circuit diagrams. The number of additional qubits needed for the discrete-velocity mesh clearly depends on the number of discrete velocities used. For example, for a $16 \times 16$ discrete-velocity mesh, we add 8 qubits (four for each direction in state space). The qubits are denoted by the "u0,"..., "v0,”... qubits in the quantum circuits. Finally, to account for solid objects, we use an additional qubit ("BC" in the diagrams) set to $\mid 1>$ to denote a cell within fluid and $\mid 0>$ for a cell within a solid. For a $64 \times 64$ Cartesian mesh and a $16 \times 16$ discrete-velocity mesh, Figure 6 shows the quantum circuit used to simulate the free-molecular flow around a rectangular body, for which the evolution of the flow field starting from an initial uniform flow is shown in Figure 7. The key feature in the quantum circuits shown in Figure 6 is the extended number of control qubits, i.e., beyond the checks on the qubits corresponding to spatial coordinates, control qubits also involve the "BC" qubit (fluid/solid flag) as well as the qubits related to the discrete-velocity indices. This least feature originates from the need to stream only data associated with selected discrete velocities in the used time-integration method.
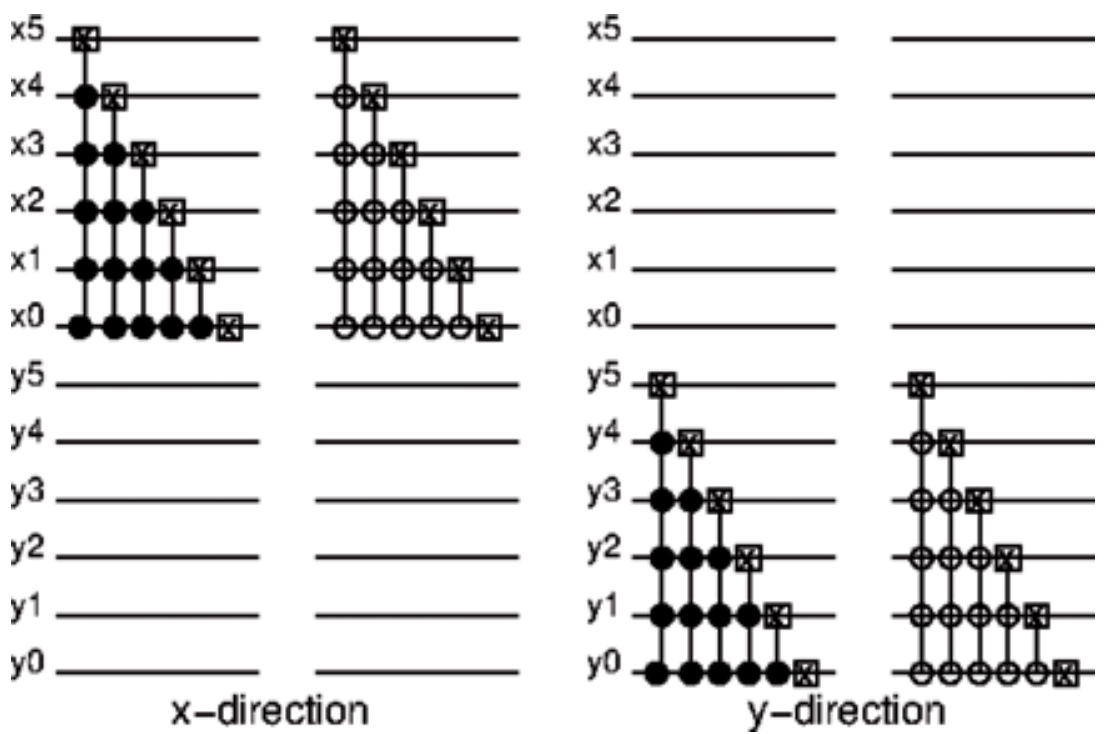

Figure 5.

Quantum circuit implementation of "left streaming" and "right streaming" in $x$-and y-direction on a $64 \times 64$ Cartesian mesh. 

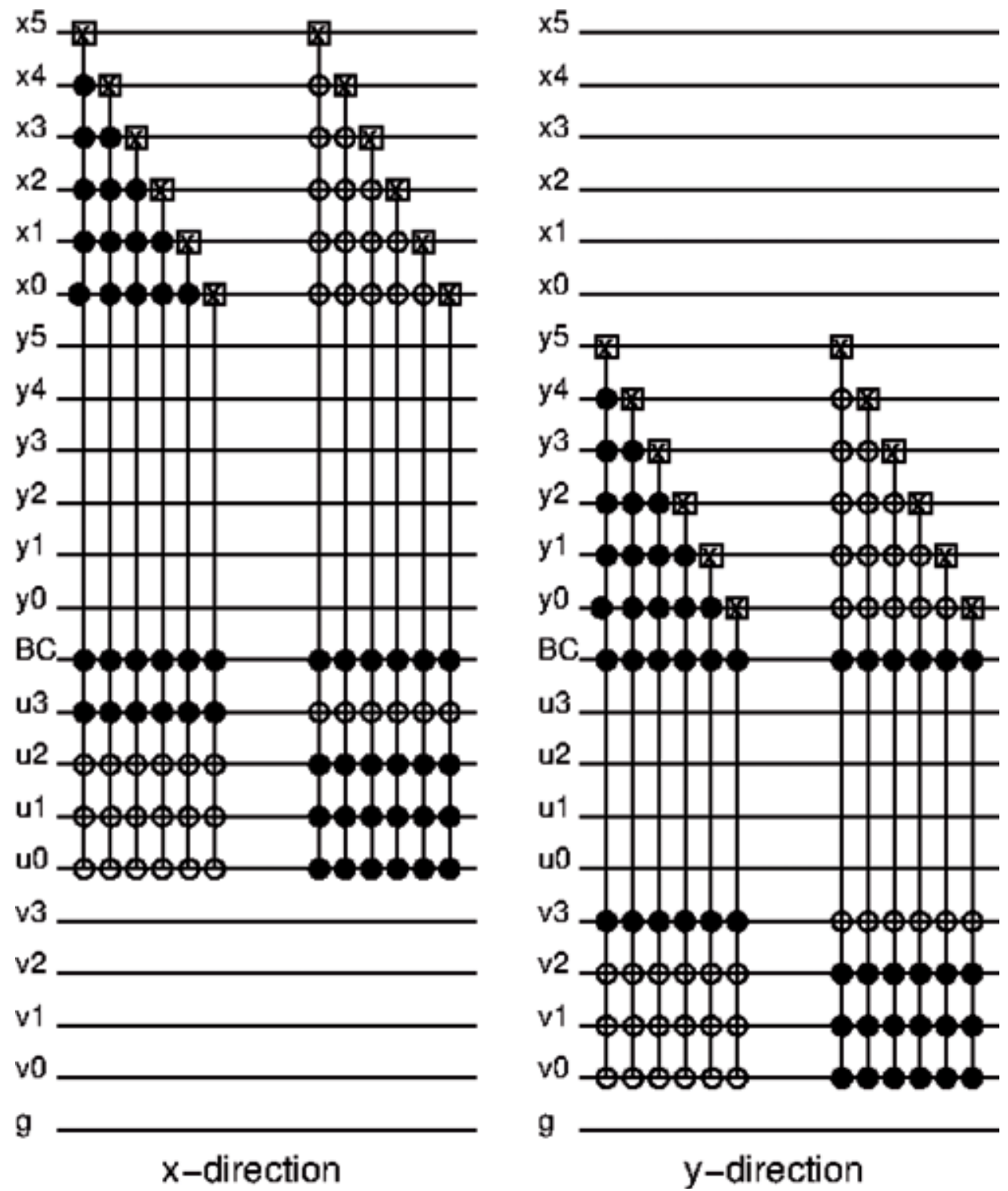

Figure 6.

Quantum circuit implementation of streaming operations for discrete-velocity method (with $16 \times 16$ velocity mesh). Two-dimensional domain with $64 \times 64$ Cartesian mesh.

\subsection{Quantum circuit implementation of specular-reflection boundary conditions}

A key aspect of the flows simulated by the quantum algorithm described here are gas-solid interactions. In this work, specular-reflection boundary conditions are assumed. This means that upon hitting a solid surface, a gas particle will bounce off this surface with the wall-normal velocity component effectively getting reversed and the tangential-flow component being preserved. In Figure 7, the time evolution of a Mach 2 free-molecular flow is shown, starting from an initial flow at uniform velocity everywhere.

As can be seen in Figure 7, the specular-reflection boundary conditions gradually make the velocity vectors align with the solid wall such that there is no longer a flow into the solid body. This is the physically correct behavior. In the quantum register, 


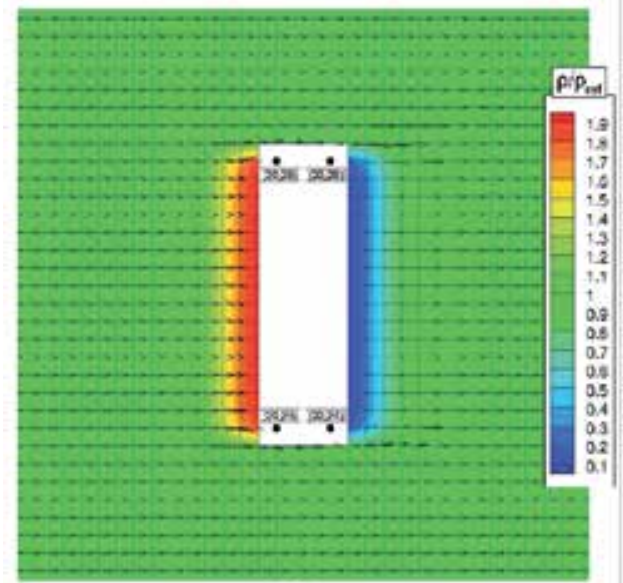

99 time steps

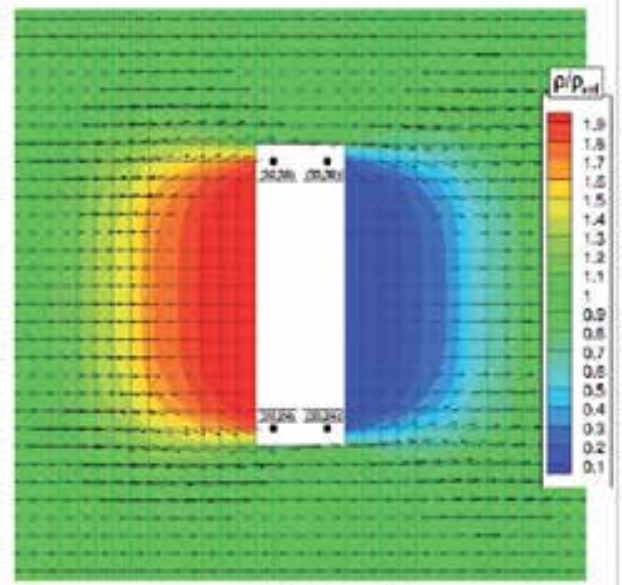

307 time steps

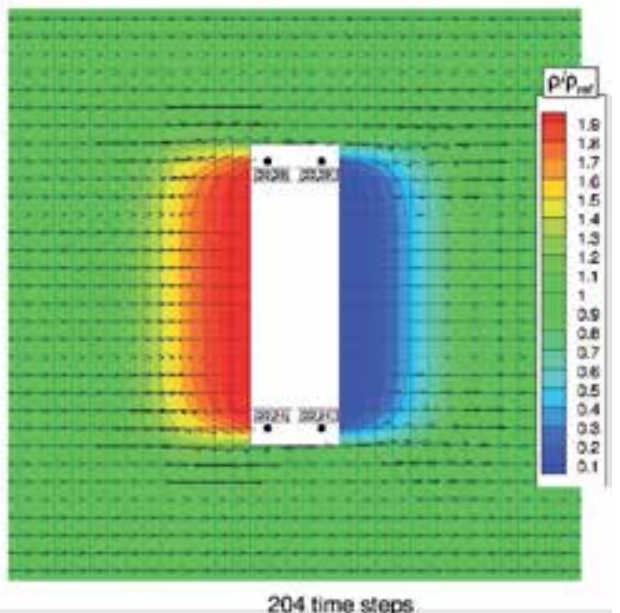

204 time steps.

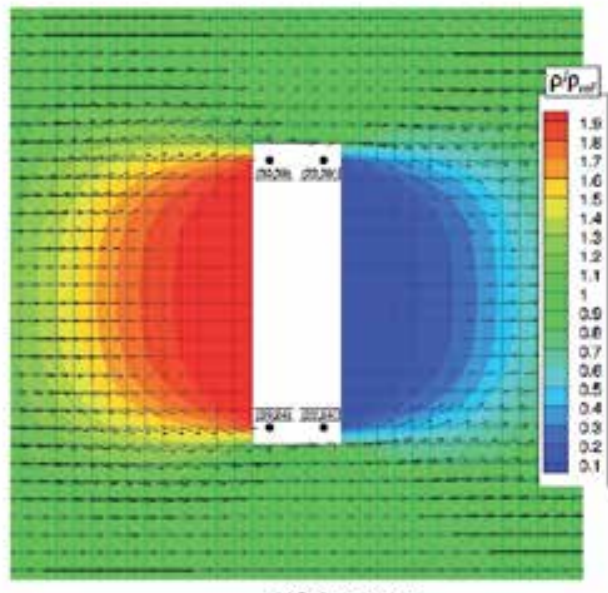

412 time steps

Figure 7.

Discrete-velocity simulation of Mach 2 flow around rectangular body. $64 \times 64$ Cartesian mesh, velocity-space mesh with $64 \times 64$ discrete velocities.

the indexing for the velocity-mesh data is designed such that a change of the sign of the discrete velocity implies a bit negation of qubits representing the index of the considered discrete velocity. As an example, Figure 8 shows the quantum circuit implementation of the specular reflection for a rectangular body. In this case, only $16 \times 16$ discrete velocities are used for clarity. It can be seen that control qubits are used to "select" cells in space for which to apply the boundary condition. A further control qubit involves the "BC" qubit representing the solid/fluid flag. The negation operation (" $\mathrm{X}$ ") is applied to the qubits representing the velocity-mesh index to create the "change of sign" of the considered discrete-velocity data. This circuit is shown as an illustration of the quantum algorithm design approach used here.

\subsection{Circuit implementations using ancilla qubits}

Although the circuits shown in Figure 6 perform the correct convection operations, an important practical constraint needs to be considered. For the quantum computer implementations achieved so far and those foreseen for the near future, the kind of multi-qubit-controlled NOT operations used here cannot be implemented. A small set of native gates will be available which most likely includes NOT, CNOT, and the Toffoli gate. 


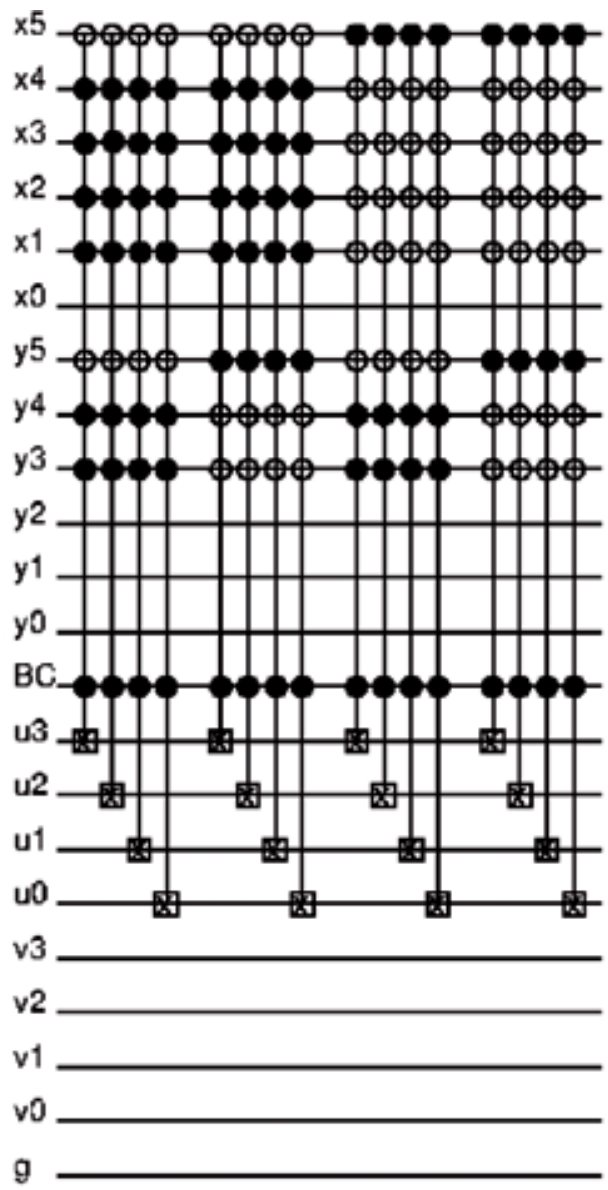

x-direction

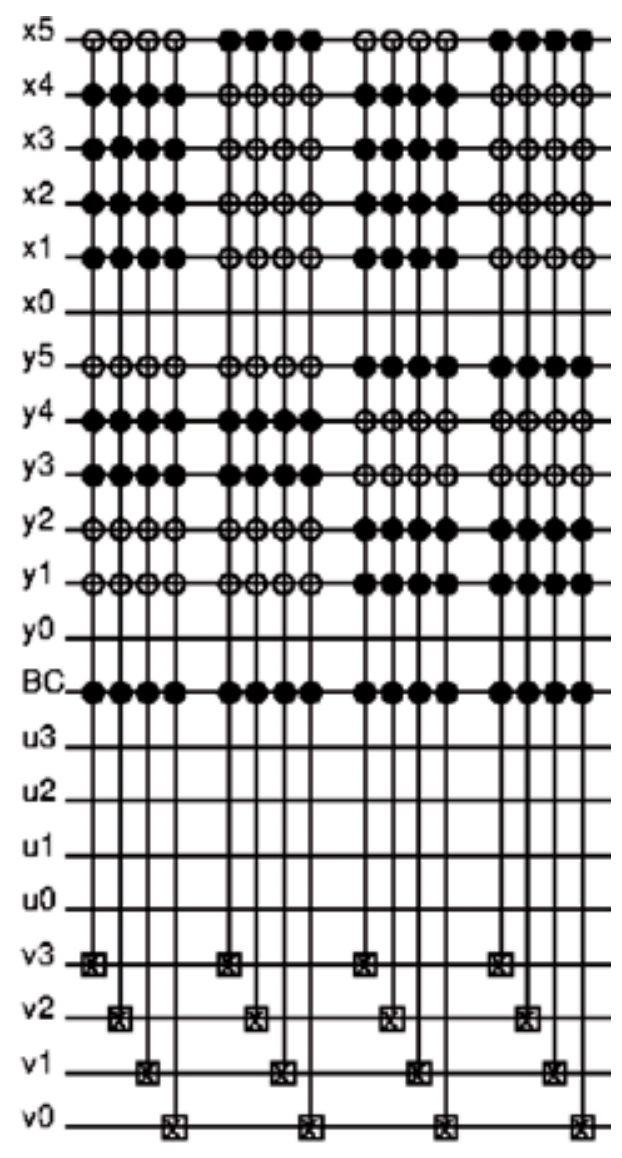

g

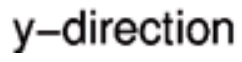

Figure 8.

Quantum circuit implementation of specular-reflection boundary conditions for rectangular body. $64 \times 64$ Cartesian mesh, $16 \times 16$ discrete-velocity mesh.

The solution around this limitation is the introduction of ancilla qubits, which can be regarded as the quantum equivalence of additional workspace in the memory of a classical computer. Then circuits involving multi-qubit operations involving a large number of control gates can be transformed into circuits with more qubits and a larger number of gate operations, however now with a smaller number of control qubits. As is typical in this context, we assume that the ancilla qubits are initially in $\mid 0>$, and since these are to be reused multiple times, the transformed circuits need to reset the ancilla qubits of this state at the end of the operations. For the quantum circuits implementing streaming in positive $x$-direction considered previously, Figure 9 shows the required circuit transformations for the cases of 1, 2, or 3 ancilla qubits. Increasing the number of qubits will make the circuits more demanding to implement, so in the actual implementation there is an important trade-off between gate complexity (i.e., within the set of native gates available) and total number of qubits. For the $64 \times 64$ two-dimensional mesh and $16 \times 16$ discrete velocities considered in Figure 6, the transformed circuits show that with three ancilla qubits, the maximum number of control gates is reduced to four in a five-qubit-controlled negation gate. However, for most practical hardware implementations, further transformations would be required. 

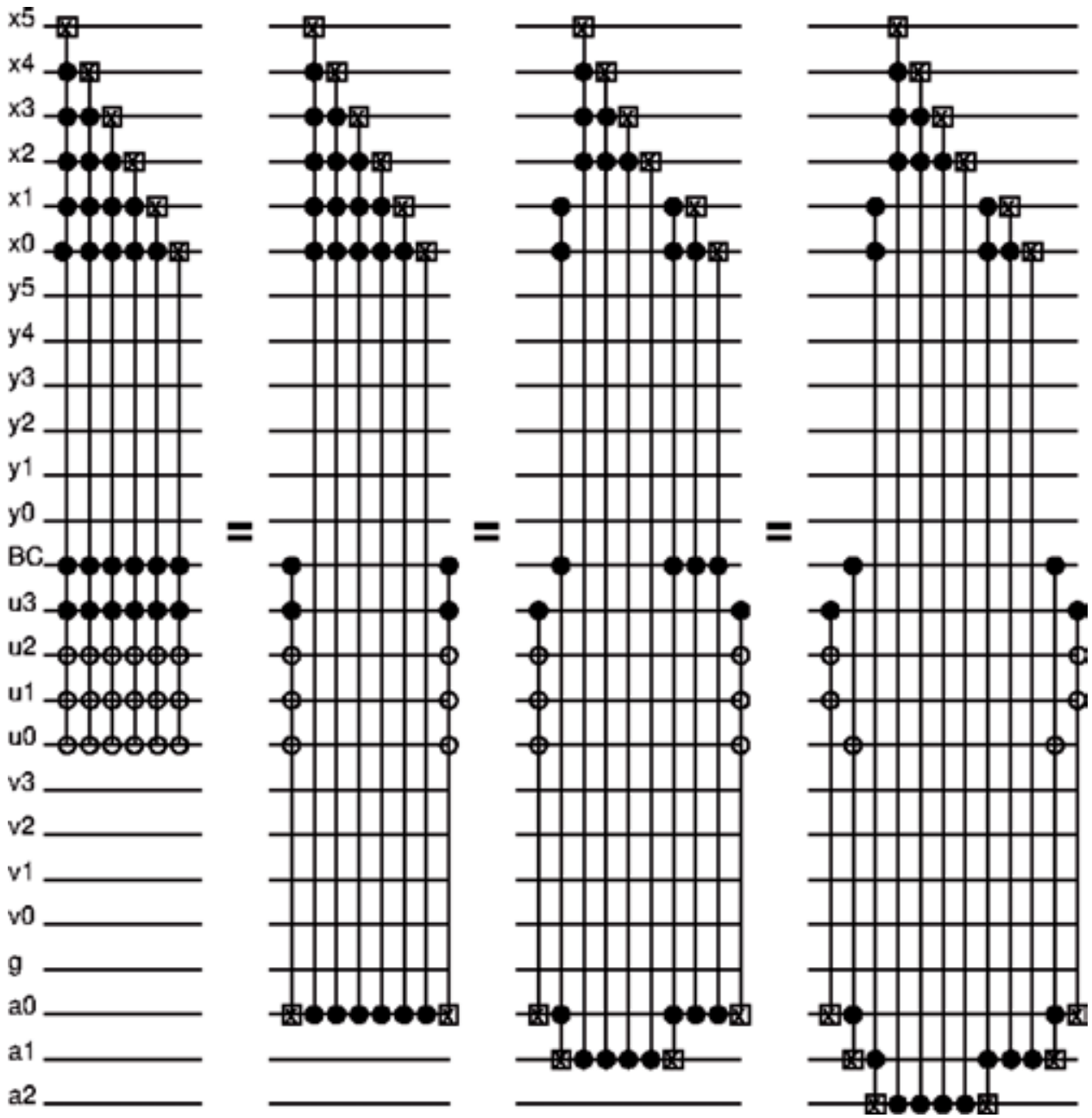

Figure 9.

Quantum circuit implementation of streaming operation. Circuit transformations are shown for addition of 1 , 2 , and 3 ancilla qubits.

\section{Conclusions}

This chapter presented two different quantum algorithms with possible applications in computational fluid dynamics. Beyond their very different areas of application, the key differences are the computational model with regard the quantum coprocessor model of quantum computing. The hybrid quantum/classical algorithm for the vortex-in-cell method involves repeated exchanges of information between classical and quantum hardware, i.e., at each time step in the time integration. In contrast, the quantum algorithm implementing a discrete-velocity method for kinetic flow modeling can be performed on the quantum processor for the duration of the simulation, with classical information exchange only required at the start and end of the simulation.

This work addressed a number of key challenges that remain to be investigated further. Firstly, the need for further efficient quantum algorithms as well as a further understanding of how to apply the quantum coprocessor model for this type of flow simulations was investigated. Secondly, the measurement-based extraction of 
classical information fundamentally changes the way quantum algorithms for CFD application will most likely be used. Finally, obtaining detailed information on the full flow field will be a challenge, so applications for which only certain characteristics of the solution are desired would present a good choice for future applications.

\section{Acknowledgements}

A part of the simulation results presented were obtained using the EPSRCfunded ARCHIE-WeSt High Performance Computer (www.archie-west.ac.uk), EPSRC grant no. EP/K000586/1.

\section{Author details}

René Steijl

CFD Laboratory, School of Engineering, University of Glasgow, Glasgow, United Kingdom

*Address all correspondence to: rene.steijl@glasgow.ac.uk

\section{IntechOpen}

(C) 2019 The Author(s). Licensee IntechOpen. This chapter is distributed under the terms of the Creative Commons Attribution License (http://creativecommons.org/licenses/ by/3.0), which permits unrestricted use, distribution, and reproduction in any medium, provided the original work is properly cited. (cc) BY 


\section{References}

[1] Nielsen MA, Chuang IL. Quantum Computation and Quantum Information: $10^{\text {th }}$ Anniversary Edition. 2nd ed. Cambridge, UK: Cambridge University Press; 2010

[2] Sinha S, Russer P. Quantum computing algorithm for electromagnetic field simulation. Quantum Information Processing. 2010;9(3):385-404. DOI: 10.1007/ s11128-009-0133-x

[3] Scherer A, Valiron B, Mau SC, Alexander S, van den Berg E, Chapuran TE. Concrete resource analysis of the quantum linear-system algorithm used to compute the electromagnetic scattering cross section of a 2D target. Quantum Information Processing. 2017;16(3):1-65. DOI: 10.1007/ s11128-016-1495-5

[4] Xu G, Daley AJ, Givi P, Somma RS. Turbulent mixing simulation via a quantum algorithm. AIAA Journal. 2018;56(2):687-699. DOI: 10.2514/1. J055896

[5] Steijl R, Barakos GN. Parallel evaluation of quantum algorithms for computational fluid dynamics. Computers \& Fluids. 2018;173:22-28. DOI: 10.1016/.compfluid.2018.03.080

[6] Harrow AW, Hassidim A, Lloyd S. Quantum algorithm for linear systems of equations. Physical Review Letters. 2009;15:150502. DOI: 10.1103/ PhysRevLett.103.150502

[7] Cao Y, Papageorgiou A, Petras I, Traub J, Kais S. Quantum algorithm and circuit design solving the Poisson equation. New Journal of Physics. 2013;15:013021. DOI: 10.1088/1367-2630/15/1/013021

[8] Britt KA, Humble TS. Highperformance computing with quantum processing units. ACM Journal on Emerging Technologies in Computing Systems. 2017;1(1):1-39. DOI: $10.1145 / 3007651$

[9] Steijl R, Barakos GN. Coupled Navier-Stokes-molecular dynamics simulations using a multi-physics flow simulation framework. International Journal for Numerical Methods in Fluids. 2010;62:1081-1106. DOI: 10.1002/fld.2641

[10] Steijl R, Barakos GN. Coupled Navier-Stokes/molecular dynamics simulations in nonperiodic domains based on particle forcing. International Journal for Numerical Methods in Fluids. 2012;69:1326-1349. DOI: 10.1002/fld.2053

[11] Cercignani C. The Boltzmann Equation and its Applications. 2nd ed. New York: Springer; 1987

[12] Vincenti WG, Kruger CH. Introduction to Physical Gas Dynamics. 2nd ed. New York: Wiley; 1967

[13] Alouges F, De Vuyst F, Le Coq G, Lorin $\mathrm{E}$. The reservoir technique: A way to make Godunuv-type scheme zero to very low diffuse. Application to Colella-Glaz solver. European Journal of Mechanics-B/Fluids. 2008;27(6):643-664. DOI: 10.1016/j. euromechflu.2008.01.001

[14] Fillion-Gordeau F, MacLean S, Laflamme R. Algorithm for the solution of the Dirac equation on digital quantum computers. Physical Review A. 2017;95(4):042343. DOI: 10.1103/ PhysRevA.95.042343 



\title{
Chapter 4
}

\section{Non-Gaussian Entanglement and Wigner Function}

\author{
Mustapha Ziane and Morad El Baz
}

\begin{abstract}
A measure of non-Gaussian entanglement in continuous variable (CV) systems based on the volume of the negative part of the Wigner function is proposed. We analyze comparatively this quantity with a numerical evaluation of the negativity of the partial transpose (NPT) considering a system of Bell states formed in the coherent state basis (quasi-Bell states).
\end{abstract}

Keywords: Wigner function, negativity, non-Gaussian state, nonclassicality, non-Gaussianity, quasi-Bell states, coherent states

\section{Introduction}

Continuous variable (CV) quantum optical systems are well-established tools for both theoretical and experimental investigations of quantum information processing (QIP) [1, 2]. Entangled states represent key resources, both for quantum computers and for many communication schemes $[1,3]$, an can be realized with Gaussian two-mode states; these states are relatively easy to work with theoretically and are also commonly produced in a laboratory. It has been successfully applied to implement various important protocols, such as quantum teleportation [4-6], quantum dense coding [7-9], and entanglement swapping [10]. This advancement comes from the development of Gaussian optical operations, such as beam splitting, phase shifting, squeezing, displacement, and homodyne detection. Recently, it became evident that the understanding of entanglement behavior beyond Gaussian systems is a necessity [11-13]. Furthermore, recent theoretical investigations have shown some limits to the Gaussian operations. For example, the no-go theorem relating to the distillation of entanglement shard by distant parties using only Gaussian local operations and classical communications (LOCC) $[14,15]$. Moreover, on the theoretical level, the study of entanglement in many-body systems has been limited to Gaussian states [16-19] where the quantification of quantum correlations $(\mathrm{QC})$ reduces to the study of the covariance matrix, but the non-Gaussian entanglement doesn't have such a simplified approach.

The problem of quantifying entanglement in non-Gaussian systems, in a way that is independent of particular external parameters, hasn't solved yet; it is our main objective in this paper. An entanglement measure $E$ of the state $\rho$ should satisfy some criteria [20] to be an entanglement monotone. Many quantities have been proposed as a quantifier of entanglement in discrete variables (DV) and CV Gaussian states. Recently, however, two entanglement measures that are much 
more amenable to evaluation have been proposed, the negativity of the partial transpose (NPT) and its logarithmic extension [21].

In this chapter, we are interested in establishing a direct measure of entanglement in non-Gaussian systems. This measure is based on the Wigner representation in the phase space of the non-Gaussian states. That is, they are defined in terms of the quantification of the degree of the negativity of Wigner function (NWF) [22, 23]. The most distinctive feature of this entanglement measure is the ease of calculated with a numerical integration.

\section{Two-mode quasi-Bell state: an entangled non-Gaussian state}

The simplest example of a non-Gaussian state is the single-photon state. There are also other examples that can be generated by excitations of Gaussian states $[24,25]$. Here we are going to use quasi-classical state that has been extensively studied for its nonclassical proprieties and violation of Bell inequalities; it is the superposition of two-mode standard coherent states (SCS). Let us consider two modes of electromagnetic fields $\mathrm{A}$ and $\mathrm{B}$ with corresponding annihilation operators $\hat{a}$ and $\hat{b}$. Two-mode coherent states are defined by $|\alpha, \beta\rangle=D_{a}(\alpha) D_{b}(\beta)|0,0\rangle$, where $|0,0\rangle$ is the two-mode vacuum state and $D_{i}(\alpha)$ is the displacement operator of the mode $i(i=A, B)$. The state $|\alpha, \beta\rangle$ can be expressed into the form

$$
|\alpha, \beta\rangle=e^{-\left(|\alpha|^{2}+|\beta|^{2}\right) / 2} \sum_{n, m}^{\infty} \frac{\alpha^{n} \beta^{n}}{\sqrt{n ! m !}}|n, m\rangle,
$$

where $\left|n_{1}, n_{2}\right\rangle$ are the two-mode Fock states. The quasi-Bell coherent states (QBS) are defined by the following superpositions of two-mode coherent states:

$$
\begin{aligned}
& \left|\psi_{ \pm}\right\rangle=N_{ \pm}(|\alpha, \beta\rangle \pm|-\alpha,-\beta\rangle), \\
& \left|\phi_{ \pm}\right\rangle=N_{ \pm}(|\alpha,-\beta\rangle \pm|-\alpha, \beta\rangle),
\end{aligned}
$$

where $N_{ \pm}=1 \sqrt{2 \exp \left(-2|\alpha|^{2}-2|\beta|^{2}\right)+2}$ is the normalization factor.

The Wigner function $\mathcal{W}(\hat{R}, \alpha, \beta)$ of the state (1) is given by

$$
\mathcal{W}(\hat{R}, \alpha, \beta)=\frac{1}{\pi} \exp \left(f\left(\alpha, q_{1}, p_{1}\right)+f\left(\beta, q_{2}, p_{2}\right)\right)
$$

where $\hat{R}=\left(q_{1}, p_{1}, q_{2}, p_{2}\right)^{T}$ is the quadrature operators vector and $f(x, y, z)=-2|x|^{2}+\sqrt{2}\left(x^{*}+x\right) y+i \sqrt{2}\left(x-x^{*}\right) z-y^{2}-z^{2}$. For the quasi-Bell entangled coherent states Eq. (2), the Wigner function is given by [26, 27].

$$
\begin{aligned}
\mathcal{W}_{Q C S}^{ \pm}(\hat{R}, \alpha, \beta)= & N_{\alpha, \beta, \pm}^{2}\left[\mathcal{W}\left(\hat{R}_{1}, \alpha, \alpha\right) \mathcal{W}\left(\hat{R}_{2}, \beta, \beta\right)\right. \\
& \pm \mathcal{W}\left(\hat{R}_{1}, \alpha,-\alpha\right) \mathcal{W}\left(\hat{R}_{2}, \beta,-\beta\right) \\
& \pm \mathcal{W}\left(\hat{R}_{1},-\alpha, \alpha\right) \mathcal{W}\left(\hat{R}_{2},-\beta, \beta\right) \\
& \left.+\mathcal{W}\left(\hat{R}_{1},-\alpha,-\alpha\right) \mathcal{W}\left(\hat{R}_{2},-\beta,-\beta\right)\right]
\end{aligned}
$$

where $\hat{R}_{1}$ and $\hat{R}_{2}$ are the quadrature operators vectors of the first and second modes and $\mathcal{W}\left(\hat{R}_{i}, x, y\right)$ is the Wigner function of one-mode coherent state with $i=1,2 ;\{x, y\}=\{ \pm \alpha, \pm \beta\}$ satisfies the normalization condition 
$\iiint \int_{\hat{R}} \mathcal{W}(\hat{R}, \alpha, \beta), d \hat{R}=1$. Hence the doubled volume of the integrated negative part of the Wigner function of the state (2) may be written as

$$
\delta\left(\psi_{ \pm}\right)=\iiint_{\hat{R}} \int\left|\mathcal{W}_{Q C S}^{ \pm}(\hat{R}, \alpha, \beta)\right| d \hat{R}-1 .
$$

By definition, the quantity $\delta$ is equal to 0 for coherent and squeezed vacuum states, for which $\mathcal{W}$ is nonnegative. In this work we shall treat $\delta$ as a parameter characterizing the properties of the state under consideration.

It is clear from expression (5) and the plot in Figure 1 that the Wigner function of the quasi-Bell state (2) is non-Gaussian. In order to characterize this non-Gaussianity, several measures of the degree of non-Gaussianity were proposed $[28,29]$. According to [29], the degree of non-Gaussianity of state $\rho$ is defined by

$$
\delta_{N G}(\rho)=S(\rho \| \tau) .
$$

where $S\left(\rho_{1} \| \rho_{2}\right)$ is the quantum relative entropy between states $\rho_{1}$ and $\rho_{2}$.

Here $\tau$ is the reference Gaussian state with the same first and second moments of $\rho$. This property of reference state $\tau$ leads to $\operatorname{Tr}[\rho \ln \tau]=\operatorname{Tr}[\tau \ln \tau]$, so that

$$
\delta_{N G}(\rho)=S(\tau)-S(\rho),
$$

where $S(\rho)$ is the Von Neumann entropy of the state $\rho$. Also $S(\tau)=h\left(d_{+}\right)+$ $h\left(d_{-}\right)$, where

$$
h(x)=\left(x+\frac{1}{2}\right) \ln \left(x+\frac{1}{2}\right)-\left(x-\frac{1}{2}\right) \ln \left(x-\frac{1}{2}\right)
$$

and $d_{ \pm}^{2}=\frac{1}{2}\left(\Delta(\delta) \pm \sqrt{\Delta(\delta)^{2}-4 I_{4}}\right)$ are the symplectic eigenvalues of the covariance matrix $\sigma$ of the reference Gaussian state $\tau$. Here $\Delta(\delta)=I_{1}+I_{2}+2 I_{3}$, where $I_{1}=\operatorname{det}(\mathbf{A}), I_{2}=\operatorname{det}(\mathbf{B}), I_{3}=\operatorname{det}(\mathbf{C})$, and $I_{4}=\operatorname{det}(\sigma)$ are the four local symplectic invariants of the covariance matrix:

$$
\sigma=\left(\begin{array}{cc}
\mathbf{A} & \mathbf{C} \\
\mathbf{C}^{\dagger} & \mathbf{B}
\end{array}\right)
$$

where

$$
\sigma_{i j}=\frac{1}{2}\left\langle\left\{R_{i}, R_{j}\right\}\right\rangle-\left\langle R_{i}\right\rangle\left\langle R_{j}\right\rangle
$$

For the considered states (2), we suppose that the two fields have the same mode $(\alpha=\beta)$; we find

$$
\begin{aligned}
\sigma_{\psi_{+}}= & \left(\begin{array}{cccc}
u_{+} & 0 & r_{+} & 0 \\
0 & v_{+} & 0 & s_{+} \\
r_{+} & 0 & u_{+} & 0 \\
0 & s_{+} & 0 & v_{+}
\end{array}\right), \\
\sigma_{\psi_{-}} & =\left(\begin{array}{cccc}
u_{-} & 0 & r_{-} & 0 \\
0 & v_{-} & 0 & s_{-} \\
r_{-} & 0 & u_{-} & 0 \\
0 & s_{-} & 0 & v_{-}
\end{array}\right),
\end{aligned}
$$




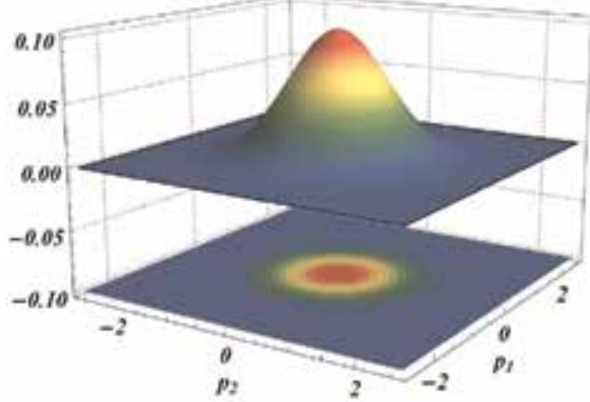

(a)

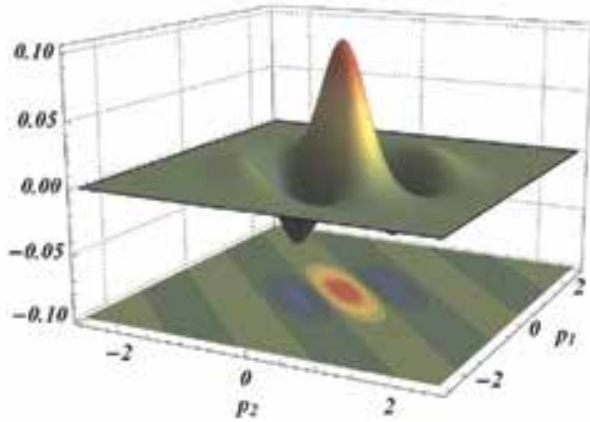

(c)

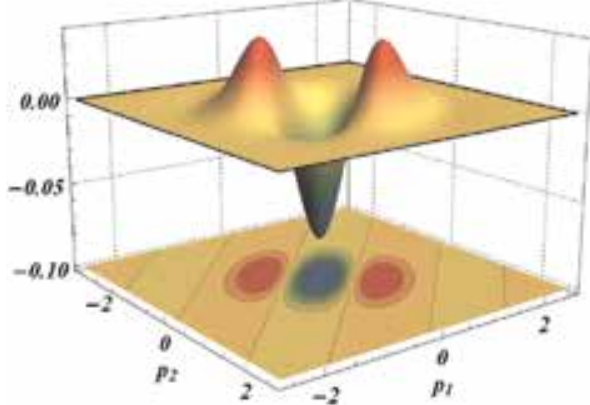

(e)

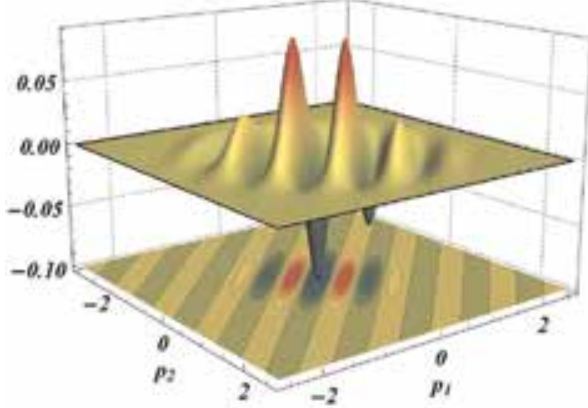

(g)

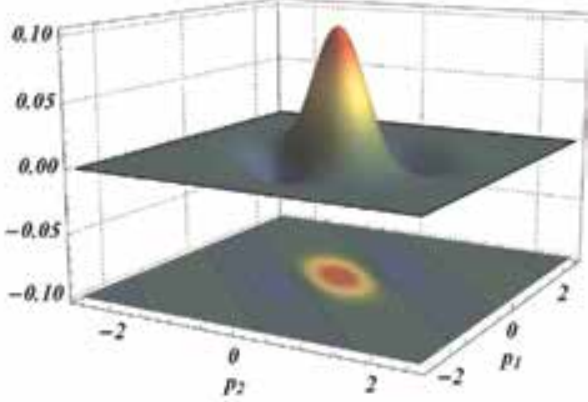

(b)

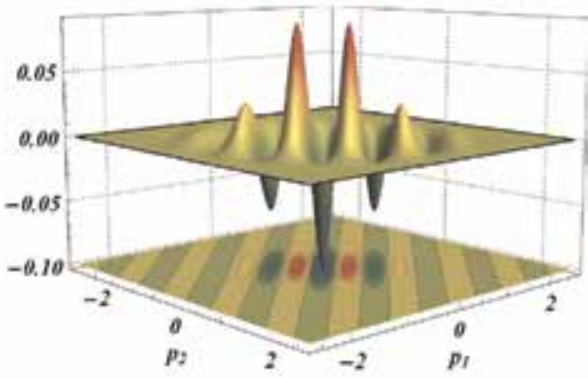

(d)

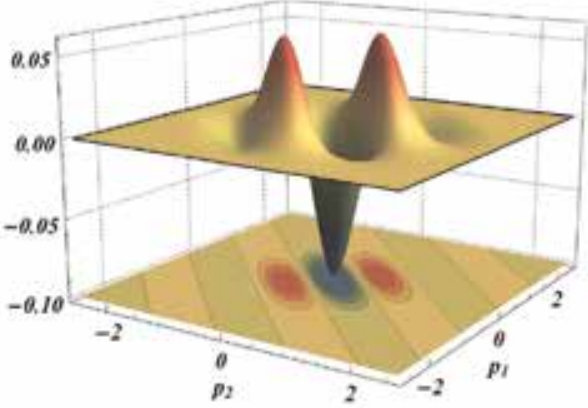

(f)

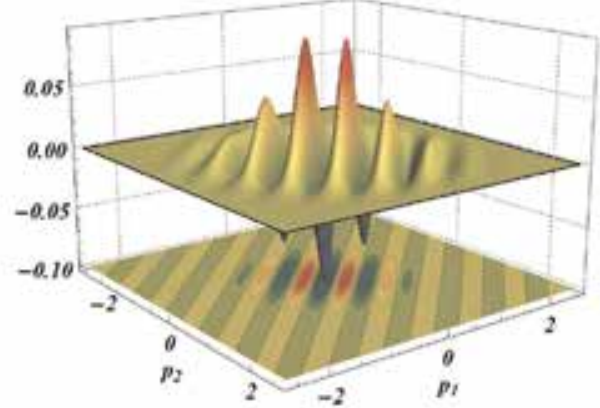

(h)

Figure 1.

Wigner function of quasi-Bell states (2). (a) Wigner function of $\psi_{+}$for $|\alpha|=0$. (b) Wigner function of $\psi_{+}$for $|\alpha|=2$. (c) Wigner function of $\psi_{+}$for $|\alpha|=1$. (d) Wigner function of $\psi_{+}$for $|\alpha|=2$. (e) Wigner function of $\psi_{-}$for $|\alpha|=0.5$. (f) Wigner function of $\psi_{-}$for $|\alpha|=1$. (g) Wigner function of $\psi_{-}$for $|\alpha|=1.5$. (h) Wigner function of $\psi_{-}$for $|\alpha|=2$. 


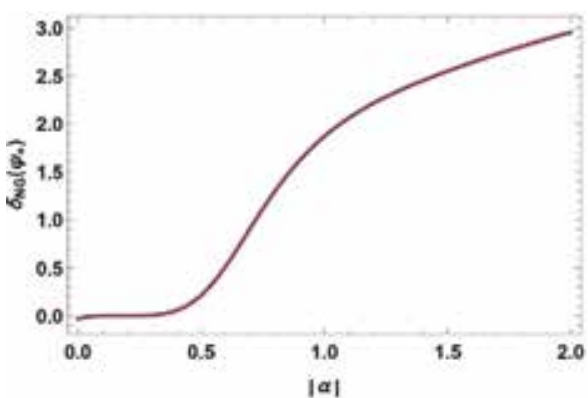

(a)

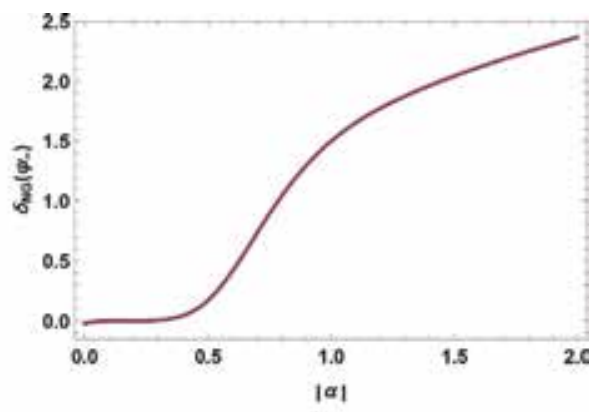

(b)

Figure 2.

Non-Gaussianity versus $|\alpha|$. (a) for states $\psi_{+}$and (b) for state $\psi_{-}$.

where we have defined

$$
\begin{array}{rr}
u_{ \pm}=N_{\alpha, \beta, \pm}^{2}\left(4 \alpha^{2} \pm \Gamma^{2}+1\right), & r_{ \pm}=4 \alpha^{2} N_{\alpha, \beta, \pm}^{2}, \\
v_{ \pm}=N_{\alpha, \beta, \pm}^{2}\left(\mp 4 \alpha^{2} \Gamma^{2} \mp \Gamma^{2}+1\right), & s_{ \pm}=\mp 4 \Gamma^{2} \alpha^{2} N_{\alpha, \beta, \pm}^{2},
\end{array}
$$

with $\Gamma=\langle\alpha \mid-\alpha\rangle=\operatorname{Exp}\left(-\frac{|\alpha|^{2}}{2}\right)$. Figure 2 shows the behavior of non-

Gaussianity of states (2) in terms of $|\alpha|$. These figures show that non-Gaussianity increases with increasing $|\alpha|$ (this behavior will be discussed in the fourth section).

\section{Numerical evaluation of negativities (NPT and NWF)}

In this section, we briefly review the NPT as a computable entanglement measure that possesses the proprieties of an entanglement monotone given in [21]. The NPT, $N(\hat{\rho})$ of a state $\hat{\rho}$ is defined as the absolute value of the sum of the negative eigenvalues of the partial transpose of $\hat{\rho}$ denoted $\hat{\rho}^{P T}$. We may write it as

$$
N(\hat{\rho})=\frac{1}{2} \operatorname{Tr}\left(\sqrt{\left(\hat{\rho}^{P T}\right)^{2}}-\hat{\rho}^{P T}\right)=\frac{\left\|\hat{\rho}^{P T}\right\|-1}{2},
$$

where $\|$.$\| denotes the trace norm [21].$

The quasi-Bell coherent state (2) is defined in a non-orthonormal basis, and it is typically not possible to obtain an analytical expression for the negativity. However, as shown in the following, one can compute it numerically. First, we expand the quasi-Bell state (2) in the Fock basis:

$$
\hat{\rho}_{ \pm}=\sum_{n_{1}, n_{2}, m_{1}, m_{2}} \rho_{n_{1}, n_{2}, m_{1}, m_{2}}^{ \pm}\left|m_{1}\right\rangle\left\langle n_{1}|\otimes| m_{2}\right\rangle\left\langle n_{2}\right|,
$$

where

$$
\rho_{n_{1}, n_{2}, m_{1}, m_{2}}^{ \pm}=N_{0}^{2} e^{-2|\alpha|}\left(\frac{\alpha^{\left(n_{1}+n_{2}\right)}}{\sqrt{n_{1} ! n_{2} !}}+\frac{(-\alpha)^{\left(m_{1}+m_{2}\right)}}{\sqrt{m_{1} ! m_{2} !}}\right) .
$$


The partial transpose of this state with respect to mode two is

$$
\hat{\rho}_{ \pm}=\sum_{n_{1}, n_{2}, m_{1}, m_{2}} \rho_{n_{1}, m_{2}, m_{1}, n_{2}}^{ \pm}\left|m_{1}\right\rangle\left\langle n_{1}|\otimes| m_{2}\right\rangle\left\langle n_{2}\right| \text {. }
$$

The eigenvalues are obtained by numerical diagonalization of the partial transpose density matrix (19). With this result, we can obtain the NPT straightforwardly using Eq. (16), and Figure 3a and $\mathbf{b}$ shows the numerical values of this NPT.

\section{Discussion}

In this section, we will discuss the different behaviors of the non-Gaussian entanglement and the variation of the negativity of the WF for the bipartite system considered early in terms of the coherent state amplitude $|\alpha|$.

Figure 2 shows the variation of the degree of non-Gaussianity for the states in Eq. (2) as a function of coherent state amplitude $|\alpha|$. We see that the nonGaussianity $\delta_{N G}$ measured by (8) equal to 0 for small values of $|\alpha|$ increases with increasing values of the parameter $|\alpha|$ to larger values much higher than 1 and does not establish in a maximum value. On the other hand, the NPT plots are shown in Figure $3 \mathbf{a}$ and $\mathbf{b}$ for the state (2) equal to 0 for $|\alpha|=0$ and increase with increasing values of the parameter $|\alpha|$ to reach its maximum value that is, equal to 1 for $|\alpha| \gtrsim 1$.3. Furthermore, it is seen that the entanglement for large values of $\alpha$ reaches its maximum value. It is worthwhile noting that, at the limit of large values of the parameter $\alpha$, the coherent states $|\alpha\rangle$ and $|-\alpha\rangle$ become orthogonal; thus the behavior of quasi-Bell state (2) is, as expected, exactly that of the Bell state.

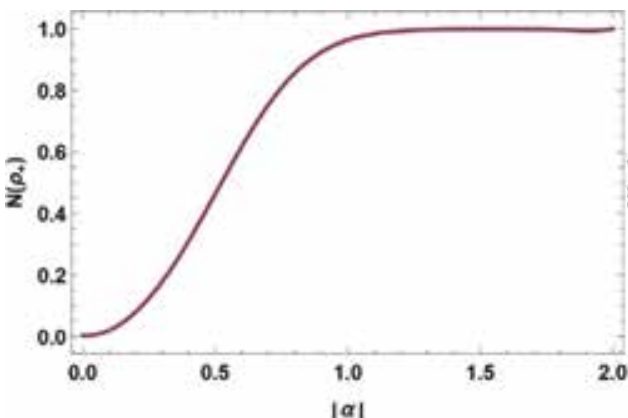

(a)

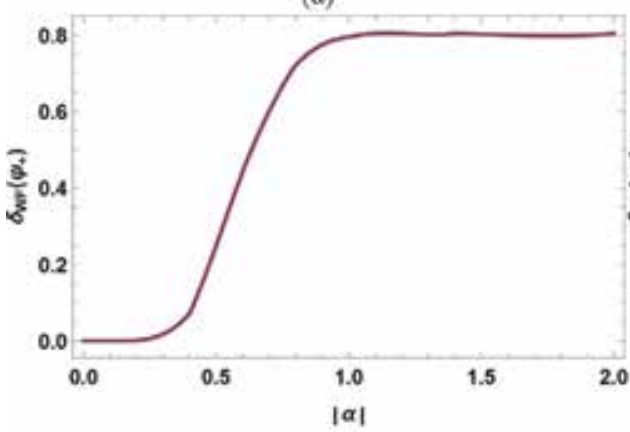

(c)

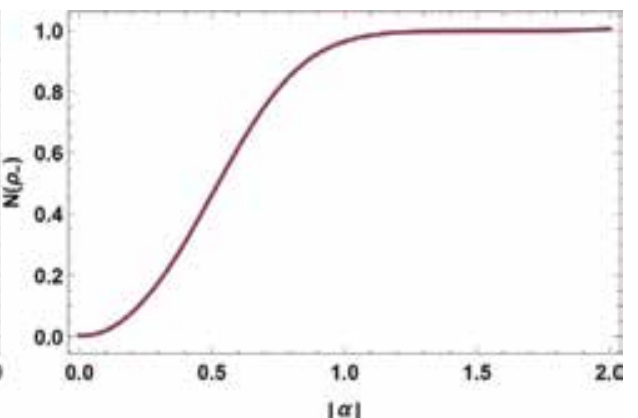

(b)

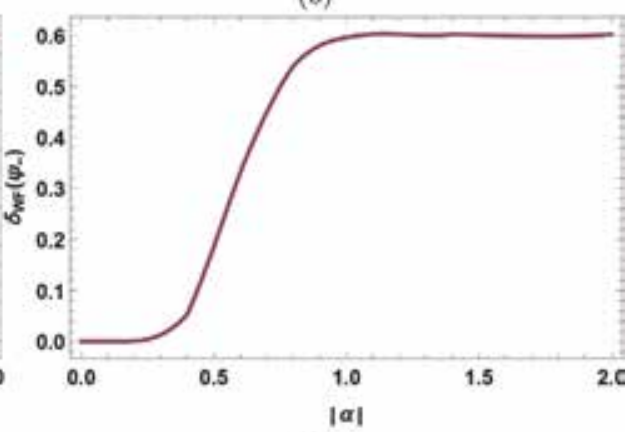

(d)

Figure 3.

Negativity of the partial transpose versus $|\alpha|$ : for the quasi-Bell state $\psi_{+}(a)$ and $\psi_{-}(b)$. Negativity of the Wigner function versus $|\alpha|$ : for the quasi-Bell state $\psi_{+}(c)$ and $\psi_{-}(d)$. 
The plot in Figure 3c and $\mathbf{d}$ shows the behavior of the NWF as a function of $|\alpha|$ for the non-Gaussian system (2). These two plots show that the NWF $\delta_{W F}$ has the same behavior as the NPT. This allows to show that they behave identically and they have the same inflection points. Which confirms that the NWF is a direct computable measure of non-Gaussian bipartite entanglement that posses the proprieties of an entanglement quantifier [21].

For our measure, $1 \geq \delta_{W F} \geq 0$, equal to zero when $\alpha$ became null and the state in Eq. 2 is now nothing but a two-vacuum product state, and it is maximal for large values of $\alpha$ where the state (2) is maximally entangled (Bell state).

\section{Conclusion}

In this work, we have evaluated the negativity of Wigner function and the negativity of the partial transpose in non-Gaussian states formed by two modes of field coherent states. We have shown that the negative parts of the Wigner function can be used as a detector of non-Gaussian entanglement. Interestingly, as used in this work, the degree of Wigner function negativity can be used as a direct quantifier of non-Gaussian bipartite entanglement.

This work allows us to describe the best characterization of the non-Gaussian Wigner function and the important use of its negativity in bipartite non-Gaussian systems, which gives more efficiency in CV quantum information theory, particularly in quantum computing [30], because the Wigner function can be measured experimentally [31,32], including the measurements of its negative values [33]. The interest put on such experiments has triggered a search for operational definitions of the Wigner functions, based on the experimental setup [34, 35]. It does represent a major step forward in the detection and the quantification of non-Gaussian entanglement in bipartite systems.

\section{Author details}

Mustapha Ziane* and Morad El Baz

Faculté des Sciences, Université Mohammed 5, Rabat, Morocco

*Address all correspondence to: mustapha.ziane@um5s.net.ma

\section{IntechOpen}

(C) 2019 The Author(s). Licensee IntechOpen. This chapter is distributed under the terms of the Creative Commons Attribution License (http://creativecommons.org/licenses/ by/3.0), which permits unrestricted use, distribution, and reproduction in any medium, provided the original work is properly cited. (c) BY 


\section{References}

[1] Nielsen MA, Chuang I. Quantum Computation and Quantum Information. 10th Anniversary Edition. New York, NY: Cambridge University Press; 2011

[2] Pati AK, Braunstein SL. Quantum information with continuous variables. Deutsch-Jozsa Algorithm for Continuous Variables. Dordrecht: Springer; 2003. pp. 31-36

[3] Horodecki R, Horodecki P, Horodecki M, Horodecki K. Quantum entanglement. Reviews of Modern Physics. 2009;81(2):865

[4] Braunstein SL, Kimble HJ. Teleportation of continuous quantum variables. Physical Review Letters. 1998; 80(4):869

[5] Furusawa A, Sørensen JL, Braunstein SL, Fuchs CA, Kimble HJ, Polzik ES. Unconditional quantum teleportation. Science. 1998;282(5389):706-709

[6] Zhang TC, Goh KW, Chou CW, Lodahl P, Kimble HJ. Quantum teleportation of light beams. Physical Review A. 2003;67:033802

[7] Bowen WP, Treps N, Buchler BC, Schnabel R, Ralph TC, Bachor H-A, et al. Experimental investigation of continuous-variable quantum teleportation. Physical Review A. 2003; 67:032302

[8] Li X, Pan Q. Quantum dense coding exploiting a bright Einstein-PodolskyRosen beam. Physical Review Letters. 2002;88:047904

[9] Mizuno J, Wakui K, Furusawa A, Sasaki M. Experimental demonstration of entanglement-assisted coding using a two-mode squeezed vacuum state. Physical Review A. 2005;71(1):012304

[10] Takei N. High-fidelity teleportation beyond the no-cloning limit and entanglement swapping for continuous variables. Physical Review Letters. 2005;94:220502

[11] Gomes R, Salles A, Toscano F, Ribeiro PS, Walborn S. Quantum entanglement beyond Gaussian criteria. Proceedings of the National Academy of Sciences. 2009;106(51):21517-21520

[12] Strobel H, Muessel W, Linnemann D, Zibold T, Hume DB, Pezzè L, et al. Fisher information and entanglement of non-Gaussian spin states. Science. 2014; 345(6195):424-427

[13] Walschaers M, Fabre C, Parigi V, Treps N. Entanglement and wigner function negativity of multimode nonGaussian states. Physical Review Letters. 2017;119(18):183601

[14] Eisert J, Scheel S, Plenio MB. Distilling gaussian states with Gaussian operations is impossible. Physical Review Letters. 2002;89(13):137903

[15] Giedke G. Characterization of Gaussian operations and distillation of Gaussian states. Physical Review A. 2002;66:032316

[16] Abdul-Rahman H, Nachtergaele B, Sims R, Stolz G. Entanglement dynamics of disordered quantum xy chains. Letters in Mathematical Physics. 2016;106(5):649-674

[17] Eisert J, Cramer M, Plenio MB. Colloquium: Area laws for the entanglement entropy. Reviews of Modern Physics. 2010;82(1):277

[18] Lami L, Serafini A, Adesso G. Gaussian entanglement revisited. New Journal of Physics. 2018;20(2):023030

[19] Nachtergaele B, Sims R, Stolz G. An area law for the bipartite entanglement of disordered oscillator systems. Journal of Mathematical Physics. 2013;54(4):

042110 
[20] Vidal G. Entanglement monotones. Journal of Modern Optics. 2000;47:355

[21] Vidal G, Werner RF. Computable measure of entanglement. Physical Review A. 2002;65(3):032314

[22] Ziane M, El Baz M. Direct measure of genuine tripartite entanglement independent from bipartite constructions. Quantum Information Processing. 2018;17(8):196

[23] Ziane M, Siyouri F-Z, Elbaz M, Hassouni Y. The negativity of partial transpose vs the negativity of wigner function. International Journal of Geometric Methods in Modern Physics. 2019

[24] Agarwal GS, Tara K. Nonclassical properties of states generated by the excitations on a coherent state. Physical Review A. 1991;43:492-497

[25] Agarwal GS, Puri RR, Singh RP. Vortex states for the quantized radiation field. Physical Review A. 1997;56: 4207-4215

[26] Sen AK. Phase distribution in a disordered chain and the emergence of a two-parameter scaling in the quasiballistic to the mildly localized regime. Modern Physics Letters B. 1997; 11(13):555-564

[27] Jiang N-Q. The n-partite entangled wigner operator and its applications in Wigner function. Journal of Optics B: Quantum and Semiclassical Optics. 2005;7(9):264

[28] Genoni MG, Paris MG, Banaszek K. Measure of the non-Gaussian character of a quantum state. Physical Review A. 2007;76(4):042327

[29] Genoni MG, Paris MG. Quantifying non-Gaussianity for quantum information. Physical Review A. 2010; 82(5):052341
[30] Forcer T, Hey A, Ross D, Smith P. Superposition, entanglement and quantum computation. Quantum Information and Computation. 2002; 2(2):97-116

[31] Smithey D, Beck M, Raymer M, Faridani A. Measurement of the wigner distribution and the density matrix of a light mode using optical homodyne tomography: Application to squeezed states and the vacuum. Physical Review Letters. 1993;70(9):1244

[32] Banaszek K, Radzewicz C, Wódkiewicz K, Krasiński J. Direct measurement of the Wigner function by photon counting. Physical Review A. 1999;60(1):674

[33] Kenfack A, yczkowski K. Negativity of the wigner function as an indicator of nonclassicality. Journal of Optics B: Quantum and Semiclassical Optics. 2004;6(10):396

[34] Lougovski P, Solano E, Zhang Z, Walther H, Mack H, Schleich W. Fresnel representation of the Wigner function: An operational approach. Physical Review Letters. 2003;91(1): 010401

[35] Leonhardt U. Measuring the Quantum State of Light. Vol. 22. Cambridge University Press; 1997 

Section 3

\section{Praxiological Studies of Quantum Communication}





\title{
Quantum Walks in Quasi-Periodic Photonics Lattices
}

\author{
Dan Trung Nguyen, Daniel A. Nolan and Nicholas F. Borrelli
}

\begin{abstract}
We present construction rules for a new class of quasiperiodic photonics lattices (QPL) to realize localized quantum walks (LQWs) deterministically. The new quasiperiodic structures are constructed symmetrically with Fibonacci, Thue-Morse, and other quasiperiodic sequences. Our construction rules allow us to build the symmetrical quasiperiodic photonics lattices. As a result, LQWs with symmetrical probability distributions can be realized in these QPLs. Furthermore, the proposed QPLs are composed with different waveguides providing both on- and off-diagonal deterministic disorders. We show LQWs in the proposed QPLs are highly programmable and controllable.
\end{abstract}

Keywords: quantum walks, localized quantum walks, photonics lattices, quasiperiodic, Fibonacci sequence, Thue-Morse sequence

\section{Introduction}

Quantum walks (QWs) have been used to construct exponential speedup quantum algorithms [1-3] and quantum simulations [4, 5], to implement universal gates for quantum computers [6,7], etc. For the last few decades, scientists have made tremendous progress on research and development of those areas which are the most promising for solving problems that are intractable by classical computers. Among different schemes (or approaches) quantum photonics has the advantage of highly advanced technology that can easily generate and manipulate almost any desired photons-the walkers, even in room-temperature conditions. Discrete-time QWs (DTQW) have been demonstrated using beam splitter arrays [8,9], and continuous-time quantum walks (CTQWs) have been investigated both theoretically and experimentally in evanescently coupled parallel waveguide arrays [10-17]. Integrated photonics lattices consisting of evanescently coupled waveguides are perfectly suited for investigation of CTQWs, and in fact, laser-written waveguides were the first systems used to demonstrate quantum walks on a line with coherent light [10-13]. In those photonics lattices, the walking process occurs in the region of evanescent-coupled waveguides. As a result, spacing between waveguides in those lattices is close enough, typically on the order of several micrometers to ensure evanescent coupling to occur. It is well established both theoretically and experimentally that in a uniform and/or periodic array of coupled waveguides (photonics lattices), the probability distribution of single-photon QWs spreads across the waveguide lattice by coupling from one waveguide to its neighbors in a pattern characterized by two strong "ballistic" lobes $[11,12]$. 
In contrast to the normal QWs in periodic photonics lattices (PLs), quantum walks can be localized in disordered ones. This phenomenon belongs to a more general concept commonly known as Anderson localization (AL). Moreover, AL is usually discussed in terms of coherent evolution in the presence of a randomly disordered medium. By breaking the order of structures and/or the periodicity of the QWs' evolution through spatially and temporally randomizing operations, localized QWs (LQWs) have been realized theoretically and experimentally [15-17]. For the last decade, there have been increased interests and efforts on exploration of LQWs for applications. The research works have been inspired by proposals of employing LQWs for quantum communications, such as secure transmission of quantum information [18], and quantum memory [19]. Besides, LQWs in photonics lattices are also a very effective approach to simulate the AL using quantum optics.

Anderson predicted that the wave function of a quantum particle can be localized in the presence of a static disordered potential in his famous paper [20]. As a result, evolution of a quantum particle with its dual wave-particle nature through a disordered medium can be strongly suppressed, depending on the degree of the disorder. More generally, in a static disordered medium, destructive interferences among different propagating paths of a quantum particle could generate AL. Although AL has been widely studied in quantum solid-state physics, localization of light has recently been explored with potentially important applications [21]. One unique phenomenon is that deviations from periodicity may result in higher complexity and many surprising effects would arise. For decades, such deviations have been investigated in optics in the realization of photonic quasicrystals: a class of structures made from optical elements that are arranged in different patterns but lack translational symmetry. A quasiperiodic structure is neither a periodic nor a random system, so it could be considered as an intermediate between the order and disorder systems. Furthermore, it has been demonstrated that quasiperiodic crystals can also lead to the localization of light [22-24]. Examples of such quasiperiodic structures constructed with the Fibonacci sequence include one-dimensional (1D) Fibonacci quasiperiodic dielectric multilayers [25], semiconductor quantum wells [26], 2D quasicrystals [27], and 3D quasicrystals [28].

It is important to stress here that AL has been conventionally realized in randomly disordered systems, and the effect of disorder-induced localization is quantified by averaging over a large number of realizations on many systems having the same degree of disorder. Similarly, LQWs in integrated photonics lattices have been realized on many randomly disordered waveguide lattices. The results of LQWs are averaged over all realizations in those waveguide lattices that are well controlled in a defined range of randomness of the disorder. Experimentally, it is not simple as is shown in [15]. Note that an experimental method has been proposed to determine localized modes systematically in 1D-disordered waveguide lattices [16], and the method is useful for investigating LQWs in randomly disordered lattices. As mentioned earlier, optically quasiperiodic structures or quasicrystals can provide deterministic disorders deviated from periodicity. Localization of light has been realized deterministically in those quasicrystals [23-25], and there is no need to average over a large number of structures. In that spirit, we propose a new class of quasiperiodic photonics lattices (QPL) to realize LQWs deterministically. As presented in this chapter, the new class of QPLs could be useful for quantum communication applications as proposed recently in $[18,19]$. The new quasiperiodic structures are constructed symmetrically with Fibonacci, Thue-Morse, and other quasiperiodic sequences. Benefits of using the new class of QPL for realizing LQWs are twofold: (i) LQWs can be realized deterministically and therefore are highly programmable and optimizable, and (ii) it is much simpler for realization of LQWs in comparison with random disordered systems as there is no need to do averaging over many 
realizations. Furthermore, our simple construction rules allow us to create symmetric QPLs. Consequently, LQWs with symmetric probability distributions can be realized deterministically in these structures. The proposed QPLs are simple and straightforward to make but would be potentially useful for many research areas in quantum communication as will be discussed in this chapter.

The chapter is organized as follows. Section 1 is the introduction. The new concept of QPLs based on Fibonacci and Thue-Morse sequences is described in Section 2. In Section 3, simulation results of QWs in periodic photonics lattices (PPLs) and LQWs in the new class of photonics lattices-the quasiperiodic photonics lattices (QPLs) -are presented. Section 4 is the discussion and conclusion.

\section{Periodic and quasiperiodic photonics lattices}

In this section, we will present the general construction of photonics lattices (PLs) that will be used for our numerical investigation of single-photon quantum walks (QWs). Figure 1 shows diagrams of several PLs that are the subject of our investigation. Figure 1(a) and (b) shows diagrams of two different types of PPLs: type-I consists of identical waveguide PPLs (IPPL), and type-II are lattices of two (or more) different waveguide PPLs (DPPL). Figure 1(c) and (d) shows two different QPLs, one with the Fibonacci sequence or Fibonacci QPLs (FQPL) and the other with the Thue-Morse sequence or Thue-Morse QPLs (TMQPL).

In Figure 1, the structures are arrays of single-mode (SM) waveguides having core diameter $a$, center-to-center distance between cores $d$, and index difference between core and clad $\Delta n$. In the structures composed of two different waveguides as in the cases of DPPL, FQPL, and TMQPL, each waveguide is characterized by $V$-number. For example, waveguides $A$ and $B$ are characterized by $V_{\mathrm{A}}=\pi a_{\mathrm{A}} N A_{\mathrm{A}} / \lambda$ and $V_{\mathrm{B}}=\pi a_{\mathrm{B}} N A_{\mathrm{B}} / \lambda$, respectively, where $a_{\mathrm{A}(\mathrm{B})}$ stands for core diameter and $N A_{\mathrm{A}(\mathrm{B})}$ is the numerical aperture of waveguide $A(B)$. Note that the numerical aperture $N A$ can be determined by the index difference between core and clad $\Delta n$, and we will use $\Delta n$ to characterize waveguides in our calculations. For example, the IPPL shown

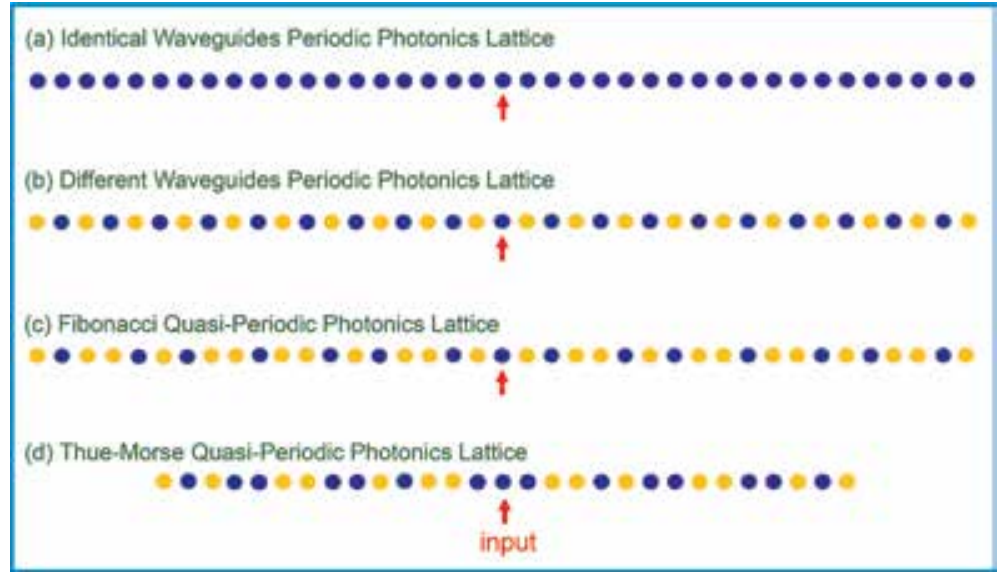

Figure 1.

Diagrams of different photonics lattices of single-mode waveguides having diameter a and center-to-center distance d. (a) IPPL with all cores have the same index difference $\triangle n$. (b) DPPL is composed of two different cores $A$ (blue) and $B$ (yellow) having $\Delta n_{A}$ and $\Delta n_{B}$, respectively. (c) Sixth-order FQPL of 39 cores composed of $A$ and $B$ waveguides. (d) Fourth-order TMQPL of 29 waveguides composed of $A$ and $B$ waveguides. The red arrows indicate the position of the input signal. The construction rules for FQPL and TMQPL are explained in the text. 
in Figure 1(a) is a regular array of 39 identical SM waveguides with $a=4 \mu \mathrm{m}$, $d=8 \mu \mathrm{m}$, and $\Delta n=0.0035$. The DPPL of 39 cores in Figure $\mathbf{1}(\mathbf{b})$ is a periodic array of two different waveguides $A$ (blue) and $B$ (yellow) with $\Delta n_{\mathrm{A}}=0.0045$ and $\Delta n_{\mathrm{B}}=0.0035$, respectively. Both $A$ and $B$ have the same $a=4 \mu \mathrm{m}$ and $d=8 \mu \mathrm{m}$. Those parameters are used in all numerical simulations in this work. Figure 1 also shows a Fibonacci QPL of 39 waveguides of sixth order and a Thue-Morse QPL of 29 waveguides of fourth order. Both FQPL and TMQPL are constructed with two different waveguides $A$ and $B$ whose properties are defined above.

Below we will describe the construction rules for our quasiperiodic photonics lattices based on Fibonacci and Thue-Morse sequences. It is worth mentioning that these rules have been first proposed to construct one-dimensional Fibonacci quasiperiodic multiple dielectric layers [22-25] and Fibonacci quasiperiodic arrays of waveguides $[29,30]$. In general Fibonacci QPLs and Thue-Morse QPLs (TMQPL) are constructed recursively with Fibonacci and Thue-Morse sequences, respectively, starting with two different single-mode waveguides $A$ and $B$. We can easily write down the formulae of the elements and their corresponding structures as in Figure 2 for the first-order elements of FQPLs and TQPLs in the upper and lower panels, respectively.

We now define a new $j^{\text {th }}$-order quasiperiodic photonics lattice as $[29,30]$

$$
F_{j}=S_{j} S_{j-1} \cdots S_{2} S_{1} S_{2} \cdots S_{j-1} S_{j},
$$

where $S_{1}, S_{2} \ldots S_{j}$ are Fibonacci and Thue-Morse elements defined in Figure 2. As an example, Figure 1(c) is a diagram of the sixth-order Fibonacci QPL with 39 cores. It is clear from Figures 1 and 2 that the photonics lattices constructed with Eq. (1), with $S j$ being the elements of Fibonacci and Thue-Morse sequences, are quasiperiodic and symmetrical.

In general, we can apply the same rules, e.g., Eq. (1), to construct symmetrical quasiperiodic photonics lattices based on other quasiperiodic sequences, such as the
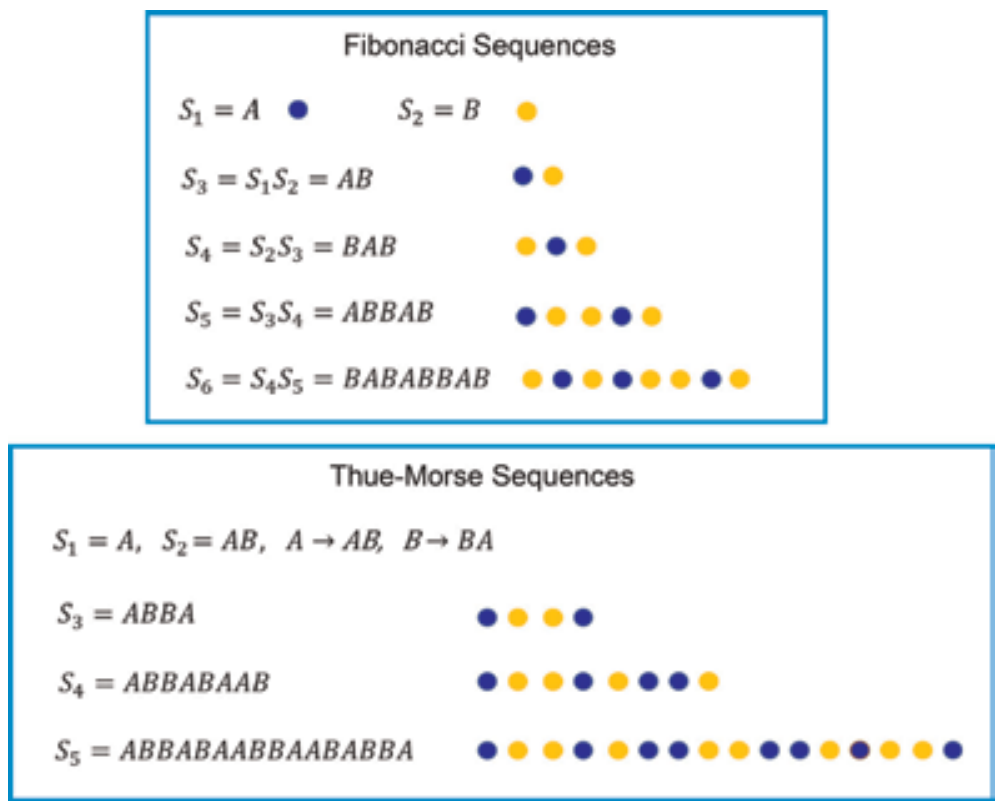

Figure 2.

First-order elements of FQPLs (upper) and TMQPLs (lower) composed by two waveguides $A$ and B. 
Rudin-Shapiro sequence, etc. Details of the new construction rules and the meaning of the deterministic disordered nature of such QPL can be seen in $[29,30]$. Next, in Section 3, we will first present the method to simulate single-photon QWs in those PLs using the beam propagation method (BPM). We will then show in detail our simulations of QWs in IPPLs and DPPLs in comparison with FQPLs and TMQPLs. Especially, we will show unique localized quantum walks in FQPLs and TMQPLs with symmetrical probability distribution.

\section{Localized quantum walks in quasiperiodic photonics lattices}

The most well-known example of classical random walks on a line consist of a walker (e.g., a particle) walking to either the left or right depending on the outcomes of an unbiased toasting coin (probability system) with two mutually exclusive results, i.e., the walker moves according to a probability distribution. At each step of the walking process, an unbiased coin is tossed, and the walker makes consecutive left-or-right decisions depending on the result of the coin (up or down), respectively. For classical random walks, it is well known that both $1 \mathrm{D}$ and 2D distributions are Gaussian distributions [31]. For the quantum version of the random walk — the quantum walks (QWs) — the main components of discrete-time quantum walks (DTQWs) are a quantum particle, "the walker," a quantum coin, evolution operators for both walker and quantum coin, and a set of observables. QWs can also be in another form that has no classical counterpart, such as the CTQWs which have been extensively investigated in photonics lattices [10-17]. In contrast to DTQWs, CTQWs (QWs for short in the following) have no coin operations, and the walking evolutions are defined entirely in a position space where continuous coupling between vertices or lattice sites is required. Integrated photonics lattices consisting of evanescently coupled 1D and 2D arrays of waveguides are perfectly suited for the investigation of QWs. In such structures, spacing between waveguides typically is on the order of several micrometers or is in strong coupling regimes for evanescent couplings to occur.

In this chapter, we will restrict ourselves to the single-photon QWs in both periodic and quasiperiodic PLs. First, we would like to emphasize that singlephoton QWs do not behave any differently from classically coherent wave propagation and the distribution of light intensity corresponds to the probability distribution of photons that can be detected [32]. It is important to stress, however, that in multiple-photon QWs (indistinguishable or entangled photons), truly nonclassical features will appear. Although single-photon QWs have limited features, the effects are still very important not only for understanding but also for quantum applications.

Let us briefly describe how single-photon QWs can be modeled mathematically and realized experimentally in PLs. Below, we follow the description from Refs. $[11,12,32]$ which are excellent references on QWs in PLs. In general, the Hamiltonian description of the problem of quantum walks in a PL can be written as

$$
H=\hbar \sum_{n}\left\{\beta_{n} a_{n}^{\dagger} a_{n}+\sum_{m} \kappa_{n m} a_{n}^{\dagger} a_{m}\right\}
$$

where $a_{n}^{\dagger}\left(a_{n}\right)$ is the creation (annihilation) operator of a photon in the $n^{\text {th }}$-waveguide, $\beta_{n}$ is the propagation constant of the $n^{\text {th }}$-waveguide, and $\kappa_{n m}$ is the coupling coefficient between nearest neighbor sites $n=m \pm 1$. The propagation of a single photon can be described by the Heisenberg equation as below: 


$$
\frac{d a_{k}^{\dagger}}{d z}=-\frac{i}{\hbar}\left[a_{k}^{\dagger}, H\right]=i\left(\beta_{k} a_{k}^{\dagger}+\kappa_{k, k+1} a_{k+1}^{\dagger}+\kappa_{k, k-1} a_{k-1}^{\dagger}\right)
$$

As described in detail in Refs. [11, 12, 32], since the system is conservative, and the Hamiltonian is explicitly time independent, we may formally integrate Eq. (3) to obtain the input-output relation for the mode operators as

$$
a_{k}^{\dagger}(z)=\sum_{j=1}^{N} U_{j, k} a_{j}^{\dagger}(0)
$$

For the special case of a single photon coupled into site $k$ of a uniform waveguide array $\left(\kappa_{k, k+1}=\kappa_{k, k-1}=\kappa\right.$, and $\left.\beta=\beta_{k}\right)$, one can show that the probability amplitude at site $j$ is analytically described by

$$
U_{k, j}=i^{(k-j)} \exp (i \beta z) J_{k-j}(2 \kappa z),
$$

where $J_{k-j}(z)$ is a Bessel function of the first kind and order $(k-j)$. When a single photon is coupled to waveguide $k$, it will evolve to waveguide $j$ with a probability $\eta_{j}=\left|U_{k, j}(z)\right|^{2}=J_{k-j}^{2}(2 \kappa z)$. From the solution of the single-photon QWs, e.g., Eq. (5), it is clear that the photon distribution spreads across the lattice by coupling from one waveguide to its neighbors in a unique pattern that is typically characterized by two strong "ballistic" lobes [12, 32].

It is worth noting that such analytical solutions, e.g., Eq. (5) above, can be obtained only in uniformly regular PLs consisting of identical SM waveguides. In general, simulations of QWs in different PLs, even in the periodic arrays that are composed of different waveguides, are not simple and become extremely difficult in irregular PLs. Simulation of LQWs in randomly disordered structures becomes more challenging even just only for the single-photon QW problems. Here we present an effective approach to simulate single-photon QWs. As stated earlier, single-photon QWs do not behave any differently from classically coherent wave propagation, and the distribution of light intensity corresponds to the probability distribution of photons. Therefore, we can use the beam propagation method (BPM), one of the most effective methods of light propagation simulation in complicated structures for simulating single-photon QWs in different and irregular PLs. The method of BPM has been well developed for decades [33, 34], and commercial software is available. Below, we describe briefly the BPM, and we show the results of BPM simulation of QWs in periodic PLs, in quasiperiodic FQPLs, and also in randomly disordered PLs.

The wave equation in paraxial approximation for the slowly varying electric field that propagates along the $z$-axis in a general structure can be written as [33-35]:

$$
\frac{d}{d z} E(x, y, z)==(\hat{D}+\hat{V}) E(x, y, z)
$$

The diffraction $\hat{D}$ and inhomogeneous operators $\hat{V}$ are given by:

$$
\hat{D}=\frac{i}{2 k}\left(\frac{\partial^{2}}{\partial x^{2}}+\frac{\partial^{2}}{\partial y^{2}}\right), \hat{V}=(i k \Delta n(x, y, z)-\alpha(x, y, z))
$$

In Eq. (7) $k=n_{0} k_{0}=\frac{n_{0} \omega}{c}=2 \pi n_{0} / \lambda$ where $n_{0}$ is the background or reference refractive index and $\lambda$ is the free-space wavelength, $\Delta n=n(x, y, z)-n_{0}$ is the 
refractive index profile relative to the reference refractive index, and $\lambda$ is the power absorption/loss of the waveguide. A small propagation step is implemented using the following approximation:

$$
E(x, y, z+\Delta z)=e^{(\hat{D}+\hat{V}) \Delta z} E(x, y, z) \approx e^{\hat{D} \Delta z / 2} e^{\hat{V} \Delta z} e^{\hat{D} \Delta z / 2} E(x, \mathrm{y}, \mathrm{z})
$$

where $\exp (\hat{D} \Delta z / 2)$ means taking a half step of diffraction alone and $\exp (\hat{V} \Delta z)$ means taking the whole step of linear propagation alone. This approximation operation is third-order accurate in the propagation step size, and the change by each step is required to be small when compared to unity. The BPM solution, e.g., Eq. (8), can be solved very effectively by fast Fourier transformation (FFT) algorithm $[33,34]$. The method has been successfully applied to simulate Er/Yb codoped multicore fiber amplifiers [35] and $\mathrm{Yb}$-doped multicore fiber lasers [36]. We have developed our own MATLAB programs, and the simulation results of singlephoton QWs in different PLs are presented in the following paragraphs.

Figure 3 shows simulation results of single-photon QWs in the photonics lattices that are described in Figure 1. Figure 3(a) and (b) shows QWs in periodic lattices: an IPPL with 39 identical SM waveguide $B(\Delta n=0.0035)$ and a DPPL with 39 SM waveguides $A(\Delta n=0.0045)$ and $B(\Delta n=0.0035)$. Figure $3(\mathbf{c})$ and (d) shows QWs in quasiperiodic lattices, sixth-order FQPL with 39 waveguides $A$ and $B$, and fourthorder TMQPL with 29 waveguides $A$ and $B$ as described in Figure 2 and Eq. (1). All waveguides have the same core size of $4 \mu \mathrm{m}$, center-to-center separation $d=8 \mu \mathrm{m}$, and $\lambda=1.55 \mu \mathrm{m}$. The simulation results of QWs for each waveguide structure in Figure 3 show in the order from bottom to top: top-view, front-view, and probability distribution of photon at the end of the walks.

The results in Figure 3 show probability distributions of photons of QWs in IPPL structures spread across the lattice by coupling from one waveguide to its neighbors in a pattern characterized by two strong "ballistic" lobes $[11,12,32]$. Interestingly, QWs in DPPLs also have those two lobes at the edges of the waveguide lattice but have no localization. Note that DPPLs are composed of two different waveguides $A$ and $B$ that are used to construct the FQPLs and TMQPLs. In contrast with periodic lattices IPPL and DPPL, the simulation results show clearly LQWs in quasiperiodic lattices FQPL and TMQPL. Furthermore, LQWs with the

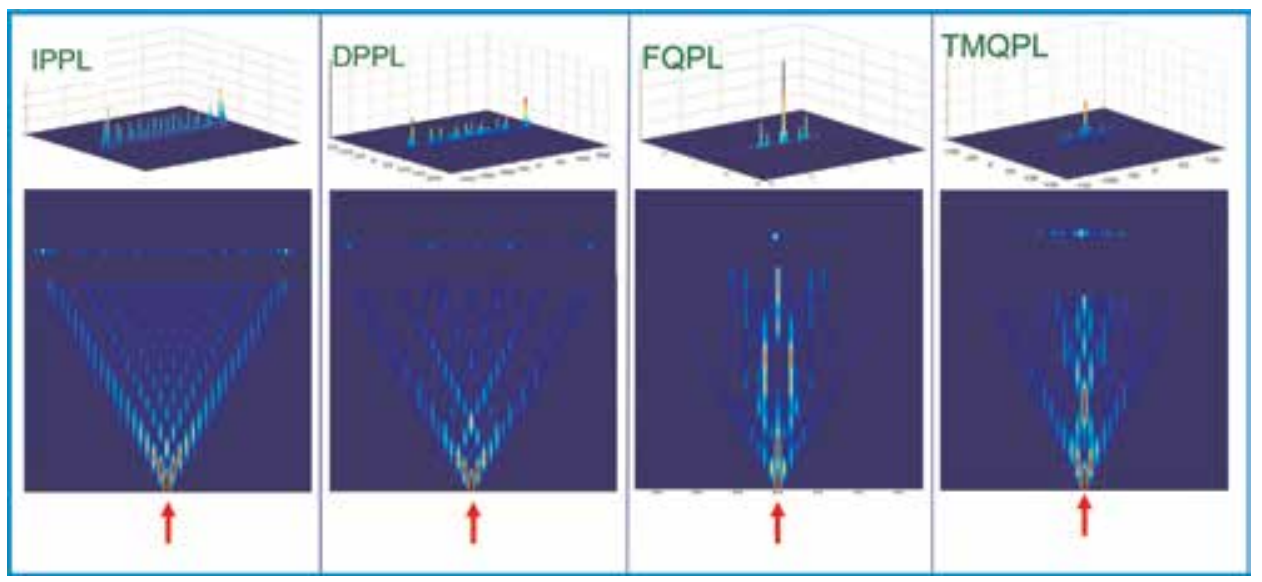

Figure 3.

QWs in periodic photonics lattices IPPL $(a)$ and DPPL $(b)$ and in quasiperiodic lattices FQPL $(c)$ and TMQPL $(d)$. From bottom to top: top-view, front-view, and probability distribution of photon (in the same scale). Lattices (a), (b), and (c) have 39 cores, and (d) has 29 cores. Red arrows indicate the position of input signal. 
symmetrical probability distribution can be realized in quasiperiodic structures constructed symmetrically, e.g., Eq. (1). It is worth noting that conventional LQWs have been realized in randomly disordered structures in which symmetrical LQWs are impossible to achieve. On the other hand, LQWs have recently been proposed for secure quantum memory applications [19]. To achieve symmetrically distributed LQWs, the authors of $[19,37]$ have proposed to use temporally disordered operations in spatially ordered systems. However, their approach requires multiple quantum coins for temporally disordered operation which could be extremely difficult in practice.

As mentioned earlier in the introduction, AL has been conventionally realized in randomly disordered systems, and the effect of disorder-induced localization is quantified by averaging over a large number of realizations on many systems having the same degree of disorder. The results of LQWs are averaged over all realizations in those waveguide lattices that are well controlled in a defined range of randomness of the disorder, and experimental realization is not simple as is shown in [15]. Meanwhile, quasiperiodic systems or quasicrystals provide deterministic disorder deviated from periodicity resulting in localization of light deterministically in those systems [22-25], meaning there is no need to do averaging over many samples or systems. Benefits of using the new class of QPL for realizing LQWs are twofold: (i) LQWs can be realized deterministically and therefore are highly programmable and optimizable, and (ii) it is much simpler for the realization of LQWs than random disordered systems as there is no need to do averaging over many realizations. Furthermore, our simple rules allow us to construct quasiperiodic FQPLs and TMQPLs symmetrically. As a result, symmetrical LQWs can be realized deterministically in these QPLs. The proposed QPLs in general, and FQPLs and TMQPLs in particular, are simple and straightforward to make but would be potentially useful for different applications in quantum communication.

Figure 4 below illustrates realizations of LQWs in randomly disordered photonics lattices. Figure 4 shows simulation results of single-photon QWs in IPPLs of 49 identical SM waveguides: IPPL without random disorder (a) and examples of QWs in IPPLs with randomly disordered waveguide positions $\Delta_{i}=0.1 d(\mathrm{~b}-\mathrm{d})$. All waveguides have the same core size of $4 \mu \mathrm{m}$, index difference between core and cladding $\Delta n=0.0035$, center-to-center separation $d=8 \mu \mathrm{m}$, and $\Delta=1.55 \mu \mathrm{m}$. Note that in Figure 4, the probability distributions of all cases are plotted in the same scale so that the differences between periodic and quasiperiodic lattices and also between the two QPLs can be clearly seen. It is worth noting that the results from BPM simulation of QWs in regular lattices of identical waveguides (IPPL) are the same as the well-known analytical solutions of Eq. (5). The BPM simulations show that photon distribution spread across the lattice by evanescent coupling from one waveguide to its nearest neighbors are characterized by two strong "ballistic" lobes $[12,32]$. Meanwhile, the results in Figure 4(b)-(d) show localizations of QWs in randomly disordered IPPLs are completely different even with the same degree of random disorder in those structures. The results of LQWs in randomly disordered IPPLs are examples of the need to do averaging over a large number of realizations for quantifying LQWs in randomly disordered systems.

As can be seen from Figures 3 and 4, LQWs in quasiperiodic structures of FQPLs and TMQPLs are controllable in contrast with the ones in spatially random disordered structures. Furthermore, LQWs with the symmetrical probability distribution can be conveniently realized in our new class of quasiperiodic photonics lattices. It is important to note that the authors in Ref. [19] have recently proposed a new scheme that employs symmetrical LQWs for storage and retrieving quantum information for quantum memory applications. The authors proposed using temporally disordered operations in spatially ordered systems to achieve symmetrically 


$$
\begin{gathered}
x_{1}^{0} x_{2}^{0} x_{3}^{0} \\
\qquad \begin{array}{l}
x_{i-1}^{0} x_{i}^{0} x_{i+1}^{0} \cdots \\
x_{i}=x_{i}^{0} \pm \Delta_{i}
\end{array}
\end{gathered}
$$

Perfect IPPL

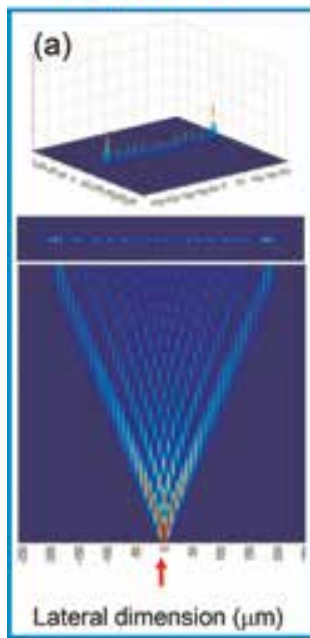

With random disorder $\Delta_{i} \leq 0.1 * d$

$\begin{array}{ll}\text { (b) } & \text { (c) }\end{array}$

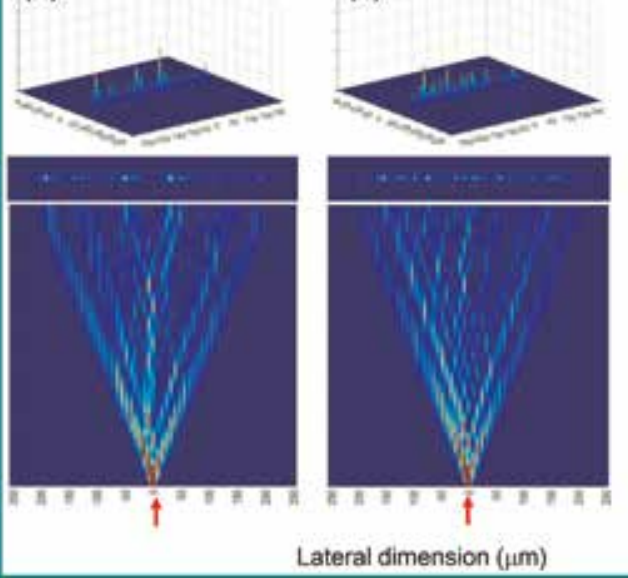

(d)
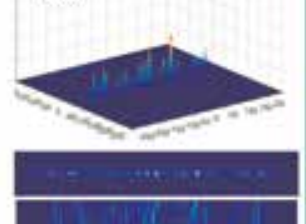

Figure 4.

QWs in IPPLs of 49 identical SM waveguides with and without spatially random disorder. (a) IPPL without disorder of waveguide positions; $(b, c, d)$ IPPLs with random disorder of positions of $10 \%$. From bottom to top: top-view, front-view, and $3 D$ probability distribution of photon (in the same scale). Upper: diagram of array of waveguides.

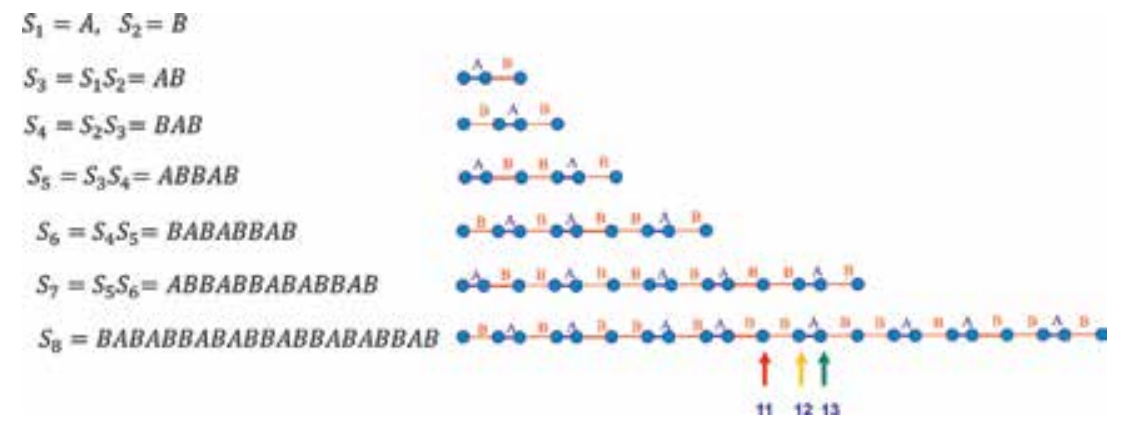

Figure 5.

Quasiperiodic elements based on the Fibonacci spacing sequence (FSS) with two fundamental distances $A$ and $B$ between nearest identical waveguides. Three arrows indicate different input cores 11, 12, and 13 used in simulation (core 1 is the first from the left).

distributed LQWs for quantum memory. However, their scheme using multiple quantum coins for temporally disordered operation would be extremely difficult in practice. As can be seen in Figures $\mathbf{4}$ and $\mathbf{5}$ in this section, symmetrical LQWS can be realized conveniently in our proposed FQPLs and TMQPLs and in other QPLs as well that can be used for similar applications.

At this point, we want to stress that localization of light has been investigated theoretically and experimentally in 1D and 2D arrays of identical waveguides that are constructed with the Fibonacci sequence in distances $[38,39]$. The quasiperiodic arrays of waveguides considered in those works are defined as the Fibonacci elements in Figure 2; however, the waveguides $A$ and $B$ were defined as the two distances 1 (unit) and $\tau=1.618$ (golden ratio of the Fibonacci sequence) between identical SM waveguides $[37,38]$. Since the quasiperiodic properties of these 
elements are determined by the Fibonacci sequences in spacing of waveguides, they can be classified as PLs with the Fibonacci spacing sequence (FSS). It is important, however, to point out that there are important differences between our proposed QPLs and the lattices of Fibonacci spacing sequence FSS in [38, 39]. The FSS lattices are constructed with the Fibonacci spacing sequence of all identical waveguides. As a result, the FSS lattices have a quasiperiodic distribution of coupling coefficients or off-diagonal deterministic disorder since all waveguides are identical. Meanwhile, our proposed QPLs are constructed with quasiperiodic sequences in general and in particular with the Fibonacci and Thue-Morse sequences with two different waveguides. Therefore, in general, our proposed QPLs, FQPLs, and TMQPLs have both propagation constants, and coupling coefficients are quasiperiodic distributions. In other words, the QPLs have both on-and off-diagonal deterministic disorders. Experimentally, localizations have been realized in on-diagonal disordered lattices [16] and in off-diagonal disordered arrays of waveguides [17]. Our proposed QPLs in general, and in particular the FQPLs and TMQPLs, would offer systems possessed both on- and off-diagonal deterministic disorders for the investigation of different properties. Furthermore, the new proposed $j^{\text {th }}$-order QPLs is constructed as an orderly sequence chain of all quasiperiodic elements up to $j^{\text {th }}$ order, and the construction rules are convenient to make QPLs symmetric as presented earlier in this section.

As an example, we show in Figure 5 the concept of quasiperiodic lattices based on FSSs with two fundamental distances $A=1$ (unit) and $B=\tau$.

In Figure 6, we show simulation results of QWs in the eighth-order FSS element with 22 waveguides for three different inputs. It is clear from Figure 6 that localizations of QWs can also be realized in this lattice. The results show the behavior of the QWs is very different depending on the input position. The results confirm
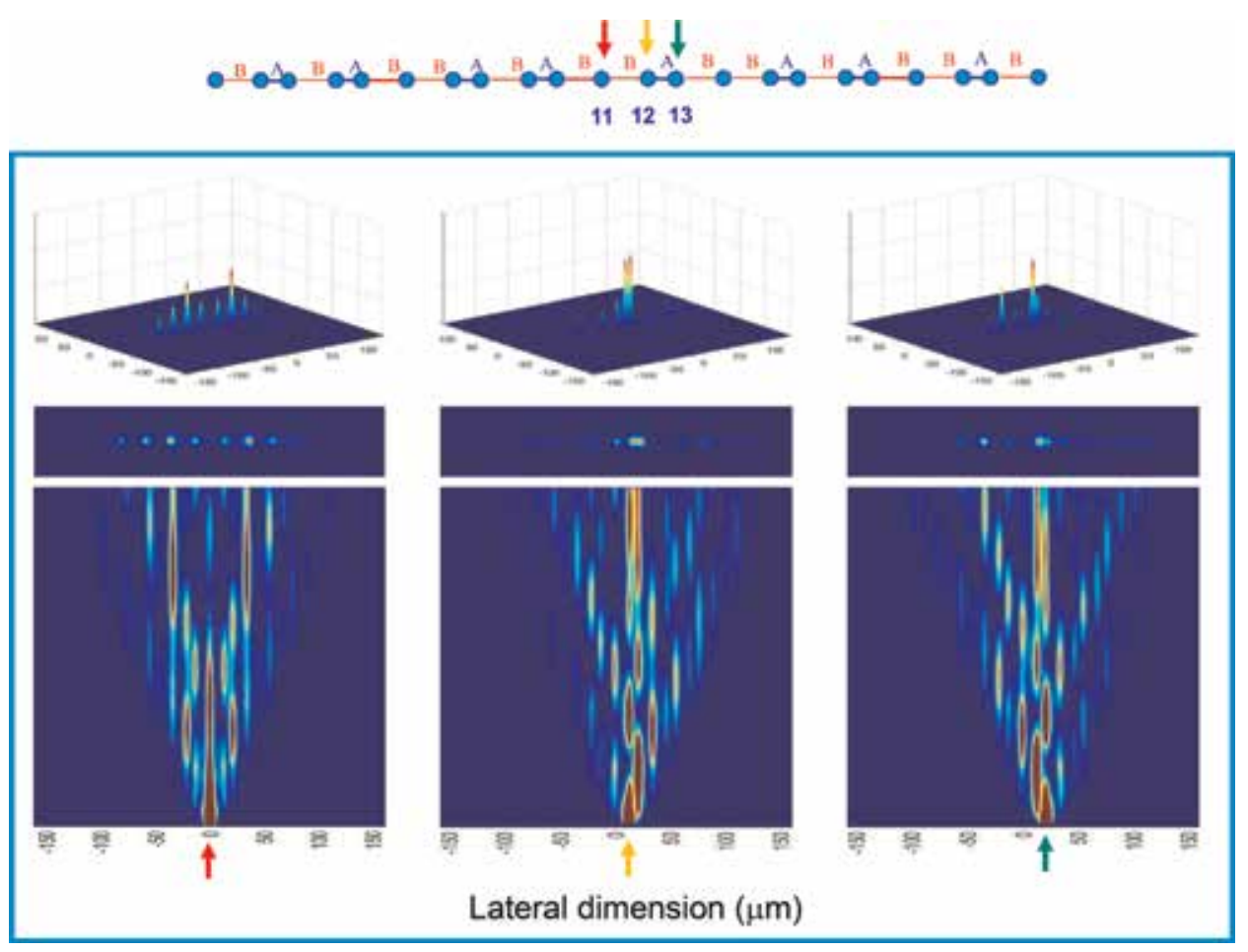

Figure 6.

LQWs in the eighth-order FSS element of 22 waveguides with different input cores as shown in diagram on top. 
important findings of our work above that LQWs can be realized in deterministic disordered QPLs.

It is worth noting that the quasiperiodic properties of the FSS elements are originated from the quasiperiodic distribution in spacing of waveguides, not because of the golden ratio $\tau$. Therefore, we do not need to restrict ourselves to the ratio $\tau=1.618$ between distances $A$ and $B$ as in $[38,39]$ for realization of localizations in these structures. This is important since we now have more freedom in choosing these two fundamental distances. Figure 7 shows results of LQWs in the eighth-order FSS element but with a different ratio $\tau=1.5,1.618$ (golden ratio), and 1.7 .

The simulation results of the eighth-order FSS element with different ratios $\tau$ show an unsymmetrical probability distribution of photons. However, the photon distribution looks quite symmetrical near the input, core-11 (core order is from the left to the right). That is because the cores are symmetrical near the input but unsymmetrical for others that are father from the input. It is worth mentioning here that the distances for completion of QW process (QW distance or $D_{\mathrm{QW}}$ for short) are slightly different for different ratios of $\tau$ as shown in Figure 7. That is because couplings between nearest cores are dependent on core-to-core distances. The results in Figure 7 are with $\tau=1.5$ and $D_{\mathrm{QW}}=4.5 \mathrm{~mm}, \tau=1.62$ and $D_{\mathrm{QW}}=4.56 \mathrm{~mm}$, and $\tau=1.7$ and $D_{\mathrm{QW}}=4.62 \mathrm{~mm}$.

Finally, the wavelength dependence of QWs in photonics lattices is well-known $[29,30]$, and that is another interesting and controllable property in such systems. We show in Figure 8 the simulation results of wavelength-dependent QWs in DPPL (left panel) and LQWs in FQPL (right panel). Note that both DPPL and FQPL in Figure 8 are composed of the same $39 A$ and $B$ waveguides, but one is periodic (DPPL), and the other is quasiperiodic lattice (FQPL).
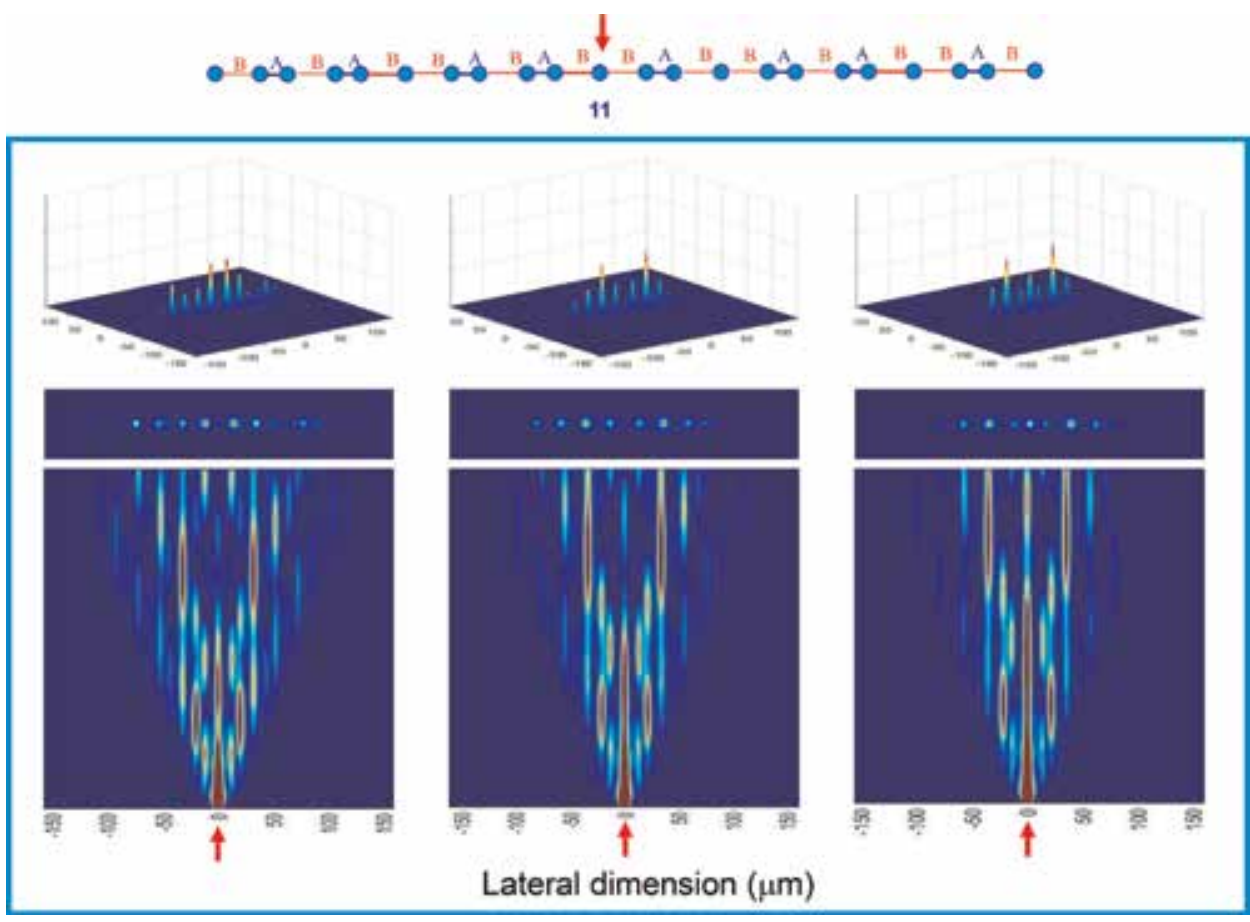

Figure 7.

LQWs in eighth-order FSS element of 22 waveguides with different ratio $\tau$ between $A$ and $B$ (core 11 is input). $\tau=1.5$ (left), 1.618 (middle), and 1.7 (right). 


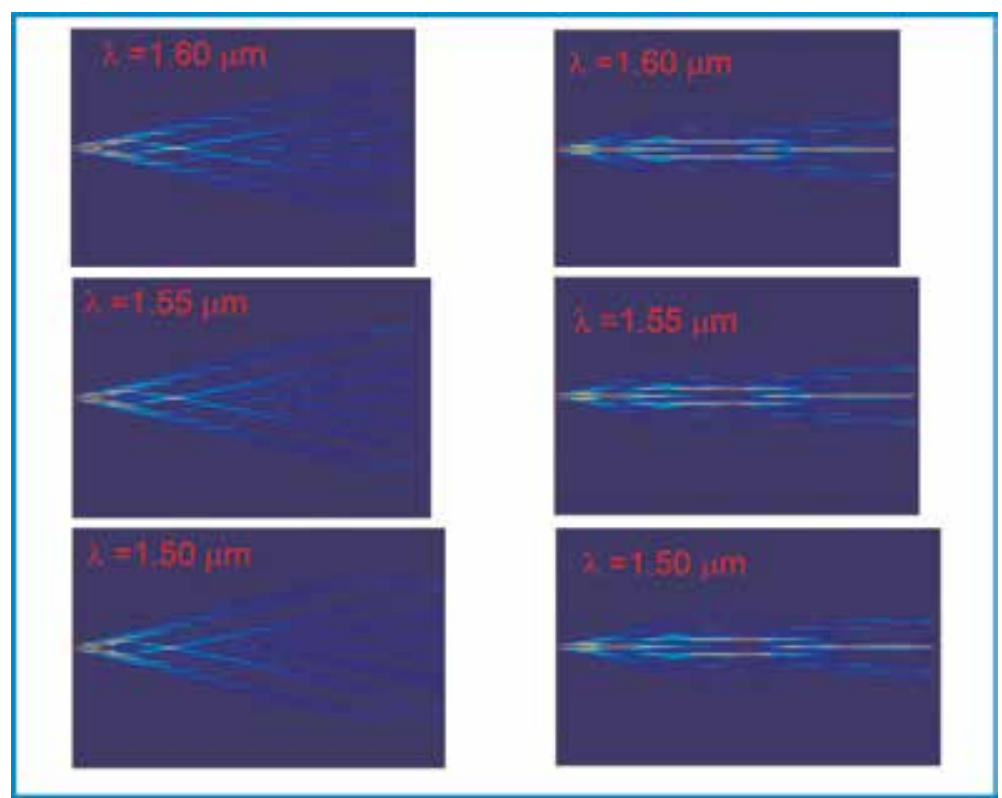

Figure 8.

Wavelength-dependent QWs in DPPL (left) and sixth-order FQPL (right). Both DPPL and FQPL are composed of the same $A$ and $B$ waveguides defined above, and both have 39 cores.

The difference between QW distances $D_{\mathrm{QW}}$ in the same structure but different wavelengths is originated from the different couplings. For the same lattice of SM waveguides, the shorter the wavelength, the weaker the evanescent couplings between the nearest cores. As a result, the light takes a longer distance to fully couple from one core to the other. This property would give us another parameter to control and optimize the QWs in both PLs and QPLs.

\section{Conclusions}

As stated earlier, QWs and LQWs are important for many applications in quantum computing and quantum communication. In particular, recent investigations show symmetrical LQWs can be potentially employed for storage and retrieve quantum information for a secure quantum memory. The authors in Ref. [19] have pointed out that symmetrical LQWs are required for quantum memory applications. Because it is impossible to achieve symmetrical LQWs in spatially randomly disordered systems, the authors proposed temporally disordered operations using multiple quantum coins. Furthermore, the concept of localization due to temporally disordered operations has been later expanded to consider multiple quantum coins with quasiperiodic sequences that include Fibonacci, Thue-Morse, and RudinShapiro sequences [37]. Although the idea of employing symmetrical LQWs for quantum memory is very interesting and worthy of further exploration, we would like to stress that experimental implementation of QWs is not simple even with only one quantum coin; therefore the idea of using multiple quantum coin operations would be extremely difficult in practice.

As presented in this chapter, our results show that symmetrical QPLs can be conveniently constructed with quasiperiodic sequences. As a result, symmetrical LQWs can be achieved in the proposed QPLs in general and in particular in FQPLs and TMQPLs. The results are significant in two aspects: 
i. Symmetrical LQWs can be achieved in QPLs. That way is much simpler than using temporal disorder due to multiple quantum coin operations.

ii. The proposed QPLs are deterministic disordered systems; therefore the systems are controllable and optimizable in contrast with randomly disordered systems.

We therefore believe that the quasiperiodic photonics lattices in general and in particular the proposed FQPLs and TMQPLs would have advantages for applications that require symmetrical LQWs, especially in comparison with the cases in which multiple quantum coins are used.

In conclusion, in this work we have presented construction rules of a new class of quasiperiodic photonics lattices constructed with Fibonacci and Thue-Morse sequences to realize deterministic LQWs. We would like to stress here that the construction rules can be applied to other quasiperiodic sequences such as RudinShapiro and other sequences to realize deterministic LQWs. Although the structures of the proposed QPLs are straightforward to make, the outcome results of LQWs are programmable and predictable, in contrast with randomly disordered systems. We have also presented an effective method of simulation of single-photon QWs in complicated structures based on beam propagation method (BPM). Furthermore, results of LQWs in the proposed symmetrical QPLs and in the Fibonacci spacing sequence (FSS) lattices are presented in detail. The unique features of QPLs that are of potential use for applications requiring symmetrical LQWs have also been discussed.

Finally, as stated earlier single-photon QWs play an important role not only for understanding the QWs effects and quantum simulations but also in many quantum applications. However, the application of single-photon states in general is limited in comparison with multiphoton states. Although our simulation results in this work are about single-photon QWs, the underlying results of our work show that symmetrical LQWs in the proposed QPLs can still be potentially important in general for quantum applications. For example, the advantage of the QPL scheme relates to the fact that the output photons localized around a particular output waveguide can be predictable and controllable with an important wavelength dependence. Also, regarding quantum communications in general, we can design the output localized around a particular waveguide using the concept of QPLs of different waveguides that can also enable low loss unique components. For example, low loss wavelength filters that are not possible with periodic structures but only with quasiperiodic structures can be realized.

\section{Conflict of interest}

The authors declare no competing financial interests and any conflict of interest.

\section{Thanks}

The authors would like to thank Kam Ng and Tyson DiLorenzo for reading the manuscript. 


\section{Author details}

Dan Trung Nguyen*, Daniel A. Nolan and Nicholas F. Borrelli

Corning Research and Development Corporation, Corning, New York, USA

*Address all correspondence to: nguyendt2@corning.com

\section{IntechOpen}

(C) 2019 The Author(s). Licensee IntechOpen. This chapter is distributed under the terms of the Creative Commons Attribution License (http://creativecommons.org/licenses/ by/3.0), which permits unrestricted use, distribution, and reproduction in any medium, provided the original work is properly cited. (c) BY 


\section{References}

[1] Ambainis A. Quantum walks and their algorithmic applications. International Journal of Quantum Information. 2003;1(4):507-518

[2] Ambainis A. Quantum walks algorithmic for element distinctness. arXiv:quant-ph. 0311001v9; 2014

[3] Montanaro A. Quantum algorithms: An overview. npj Quantum Information. 2006;2:15023

[4] Sansoni L, Sciarrino F, Vallone G, Mataloni P, Crespi A. Two-particle bosonic-fermionic quantum walk via integrated photonics. Physical Review Letters. 2012;108:010502

[5] Spring JB, Metcalf BJ, Humphreys PC, Kolthammer WS, Jin X-M, Barbieri $\mathrm{M}$, et al. Boson sampling on a photonic chip. Science. 2013;339:798-801

[6] Childs AM, Gosset D, Webb Z. Universal computation by multiparticle quantum walk. Science. 2013;339: 791-794. DOI: 10.1126/science.1229957

[7] Lovett NB, Cooper S, Everitt M, Trevers M, Kendon V. Universal quantum computation using the discrete-time quantum walk. Physical Review A. 2010;81:042330

[8] Do B, Stohler ML, Balasubramanian S, Elliott DS, Eash C, Fischbach E, et al. Experimental realization of a quantum quincunx by use of linear optical elements. Journal of the Optical Society of America B: Optical Physics. 2005; 22(2):499-504

[9] Pathak PK, Agrawal GS. Quantum random walk of two photons in separable and entangled states. Physical Review A. 2007;75:032351

[10] Peruzzo A, Lobino M, Matthews JCF, Matsuda N, Politi A, Poulios K, et al. Quantum walks of correlated photons. Science. 2010;329:1500-1503

[11] Perets HB, Lahini Y, Pozzi F, Sorel M, Morandotti R, Silberberg Y.

Realization of quantum walks with negligible decoherence in waveguide lattices. Physical Review Letters. 2008; 100:170506

[12] Bromberg Y, Lahini Y, Morandotti R, Silberberg Y. Quantum and classical correlations in waveguide lattices. Physical Review Letters. 2009;102:253904

[13] Owens O, Broome MA, Biggerstaff DN, Goggin ME, Fedrizzi A, Linjordet $\mathrm{T}$, et al. Two-photon quantum walks in an elliptical direct-write waveguide array. New Journal of Physics. 2011;13: 075003

[14] Tang H, Lin X-F, Feng Z, Chen J-Y, Gao J, Sun K, et al. Experimental twodimensional quantum walks on a photonic chip. Science Advances. 2018; 4. DOI: $10.1126 /$ sciadv.aat 3174

[15] Crespi A, Osellame R, Ramponi R, Giovannetti V, Fazio R, Sansoni L, et al. Anderson localization of entangled photons in an integrated quantum walk. Nature Photonics. 2013;7:322

[16] Lahini Y, Avidan A, Pozzi F, Sorel M, Morandotti R, Christodoulides DN, et al. Anderson localization and nonlinearity in one-dimensional disordered photonic lattices. Physical Review Letters. 2008;100:013906

[17] Martin L, Giuseppe GD, Perez-Leija A, Keil R, Dreisow F, Heinrich M, et al. Anderson localization in optical waveguide arrays with off-diagonal coupling disorder. Optics Express. 2011; 19:13636

[18] Leonetti M, Karbasi S, Mafi A, DelRe E, Conti C. Secure information 
transport by transverse localization of light. Scientific Reports. 2016;6:29918

[19] Chandrashekar CM, Busch T. Localized quantum walks as secured quantum memory. Europhysics Letters. 2015;110:10005

[20] Anderson PW. Absence of diffusion in certain random lattices. Physics

Review. 1958;109:1492

[21] Segev M, Silberberg Y, Christodoulides DN. Anderson localization of light. Nature Photonics. 2013;7:197

[22] Levine D, Steinhardt PJ. Quasicrystals: A new class of ordered structures. Physical Review Letters. 1984;53:2477

[23] Gellermann W, Kohmoto M, Sutherland B, Taylor PC. Localization of light waves in Fibonacci dielectric multilayers. Physical Review Letters. 1994;72:633

[24] Kohmoto M, Sutherland B, Iguchi K. Localization in optics: Quasiperiodic media. Physical Review Letters. 1987;58: 2436

[25] Nguyen TD, Norwood AR, Peyghambarian N. Multiple spectral window mirrors based on Fibonacci chains of dielectric layers. Optics Communication. 2010;283:4199-4202

[26] Hendrickson J, Richards BC, Sweet J, Khitrova G, Poddubny AN, Ivchenko EL, et al. Excitonic polaritons in Fibonacci quasicrystals. Optics Express. 2008;16:15382-15387

[27] Levi L, Rechtsman M, Freedman B, Schwartz T, Manela O, Segev M. Disorder-enhanced transport in photonic quasicrystals. Science. 2011; 332:1541-1544

[28] Ledermann A, Cademartiri L, Hermatschweiler M, Toninelli C, Ozin
GA, Wiersma DS, et al. Threedimensional silicon inverse photonic quasicrystals for infrared wavelengths. Nature Materials. 2006;5:942

[29] Nguyen DT, Nolan DA, Borrelli NF. Localized quantum walks in quasiperiodic Fibonacci arrays of waveguides. Optics Express. 2019;27: 886-897

[30] Nguyen DT, Nolan DA, Borrelli NF. Quantum walks in quasi-periodic arrays of waveguides. In: Proc. SPIE 10933, Advances in Photonics of Quantum Computing, Memory, and Communication XII; 109330X. 4 Mar 2019; DOI: 10.1117/12.2510176

[31] Venegas-Andraca SE. Quantum walks: A comprehensive review. Quantum Information Processing. 2012; 11:1015

[32] Gräfe M, Heilmann R, Lebugle M, Guzman-Silva D, Perez-Leija A, Szameit A. Integrated photonic quantum walks. Journal of Optics. 2016;18:103002

[33] Feit MD, Fleck JA. Light propagation in graded-index optical fibers. Applied Optics. 1978;17: 3990-3998

[34] Feit MD, Fleck JA. Computation of mode properties in optical fiber waveguides by a propagating beam method. Applied Optics. 1980;19:1154

[35] Nguyen DT, Chavez-Person A, Jiang $\mathrm{S}$, Peyghambarian N. A novel approach of modeling cladding-pumped highly Er-Yb co-doped fiber amplifiers. IEEE Journal of Quantum Electronics. 2007; 43(11):1018-1027

[36] Demir V, Akbulut M, Nguyen DT, Kaneda K, Neifeld M, Peyghambarian $\mathrm{N}$. Injection-locked, single frequency, multi-core $\mathrm{Yb}$-doped phosphate fiber laser. Scientific Reports. 2019;9:356. DOI: $10.1038 / \mathrm{s} 41598-018-36687-4$ 
[37] Gullo NL, Ambarish CV, Busch T, Dell'Anna L, Chandrashekar CM. Dynamics and energy spectra of aperiodic discrete-time quantum walks. Physical Review E. 2017;96:012111

[38] Lucic NM, Savic DMJ, Piper A, Grujic DŽ, Vasiljevic JM, Pantelic DV, et al. Light propagation in quasiperiodic Fibonacci waveguide arrays. Journal of the Optical Society of America B: Optical Physics. 2015;32(7): 1510-1513

[39] Boguslawski M, Lucic NM, Diebel F, Timotijevic DV, Denz C, Savic DJ. Light localization in optically induced deterministic aperiodic Fibonacci lattices. Optica. 2016;3(7):711-717 

Section 4

Applications and
Technologies 



\title{
Design of 4-Bit 4-Tap FIR Filter Based on Quantum-Dot Cellular Automata (QCA) Technology with a Realistic Clocking Scheme
}

\author{
Ismail Gassoumi, Lamjed Touil, Bouraoui Ouni \\ and Abdellatif Mtibaa
}

\begin{abstract}
The increasing demand for efficient signal processors necessitates the design of digital finite duration impulse response FIR filter which occupies less area and consumes less power. FIR filters have simple, regular and scalable structures. This paper represents designing and implementation of a low-power 4-tap FIR filter based on quantum-dot cellular automata (QCA) by using a realistic clocking scheme. The QCADesigner software, as widely used in QCA circuit design and verification, has been used to implement and to verify all of the designs in this study. Power dissipation result has been computed for the proposed circuit using accurate QCADesigner-E software. The proposed QCA FIR achieves about $97.74 \%$ reduction in power compared to previous existing designs. The outcome of this work can clearly open up a new window of opportunity for low-power signal processing systems
\end{abstract}

Keywords: QCA technology, QCA designer, FIR filter, low power, QCA pro

\section{Introduction}

Recently, the design of high-performance digital circuits meeting area, power and speed metrics has become a challenge. On one side, several digital signal processing applications are based on complex algorithm which requires great computational power per silicon area. On the other side, there are stringent portability and energy requirements which further complicate the design task. Therefore, achieving the required computational throughput with minimum energy consumption has become the key design goal, as it contributes to the total power budget as well as reliability of target application. So far, VLSI industry has been successfully following Moore's law. Simultaneous reduction in critical dimensions and operating voltage of CMOS transistors yields higher speed and packaging density while decreasing the silicon area and power consumption [1]. However, this trend of successive transistor scaling cannot continue for long, as the CMOS technology is reaching its fundamental physical limits and entails many challenges [2-4]. Low-power digital design is being investigated at all levels of design abstraction. 
At device level, a number of CMOS alternatives are summarized in International Technology Roadmap for Semiconductors (ITRS) report such as quantum-dot cellular automata (QCA), single-electron transistor (SET), carbon nanotube fieldeffect transistors (CNTFET) and resonant tunneling diodes (RTD) [5]. The use of (QCA) on the nanoscale has a promising future because of its ability to achieve high performance in terms of device density, clock frequency and power consumption [6-9]. Essentially, QCA offers potential advantages of ultralow-power dissipation. QCA is expected to achieve very high device density of 1012 device $/ \mathrm{cm}^{2}$ and switching speeds of $10 \mathrm{ps}$ and a power dissipation of $100 \mathrm{~W} / \mathrm{cm}^{2}$ [10]. These features, which are not offered by CMOS devices, can open new opportunities to save power in mobile systems design. In addition, they can make the proposed QCA approach useful for signal and image processing systems applied on portable communication devices where real-time processing and low-power consumption are needed in today's world in order to extend battery life. Several attempts are made towards the cost-effective realization of QCA circuit in [11-19]. Whereas QCA technology has advantages over CMOS technology, various limitations are identified. Its include placing long lines of cells among clocking zones which leads to thermal fluctuation issue and increases delay of the circuit. Recently, a universal, scalable, and efficient (USE) clocking scheme [20] is a proposed technique to overcome the mentioned limitations. This scheme can design feedback paths with different loop sizes. It is regular and flexible enough to allow placement and routing, besides avoiding thermodynamic effects due to long wires. On the other hand, for designing several digital signal processors (DSP), finite impulse response (FIR) filter is widely used as a critical component. For their guaranteed linear phase and stability, the FIR filter is used for the conception of very highly efficient hardware circuits. Theses circuits perform the key operation in various recent mobile computing and portable multimedia applications. We denote highefficiency video coding (HEVC), channel equalization, speech processing, software-defined radio (SDR) and others. Indeed, an efficient FIR filter design essentially improves the performance of a complex DSP system. This fact pushed designers to search for new methods to grant low-power consumption for FIR filter [21-28]. QCA logic design circuit is stimulated by its applications in low-power electronic design. It has lately attracted significant attention. All these above factors motivate us to investigate a new architecture around QCA by using USE clocking scheme, which can efficiently perform FIR operation.

The main concern of this paper is to present a new design for FIR filter based on QCA technology which yields significant reduction in terms of power. This paper is organized as follows. Section 2 presents the background of FIR filter structures. Section 3 indulges the preliminaries of QCA technology. Section 4 discusses the FIR filter power optimization by QCA technology. Section 5 shows the discussions and results of the proposed FIR filter-based technology. Finally, conclusions are drawn in Section 6.

\section{Background of FIR filter structures}

FIR filters are important building blocks among the various digital signal processing applications. Recently, due to the popularity of the portable batterypowered wireless communication systems, low-power and high-performance digital filter designs become more and more important.

An nth order FIR filter performs N-point linear convolution of input sequence with filter coefficients for new input sample. The transfer function of the linear invariant (LTI) FIR filter can be expressed as the following equation: 


$$
\mathrm{y}(\mathrm{n})=\sum_{k=0}^{N-1} h_{k} x(n-k)
$$

where $\mathrm{N}$ represents the length of the filter, $h_{k}$ is the Kth coefficient, and $x(n-k)$ is the input data at time instant $(n-k)$.

The $\mathrm{z}$ transform of the data output is

$$
Y(z)=H(z) \cdot X(z)
$$

where $\mathrm{H}(\mathrm{z})$ is the transfer function of the filter, given by

$$
H(Z)=\sum_{k=0}^{N} h_{k} Z^{-k}
$$

Several architectures have been proposed in the last recent years. A filter can be implemented in direct form (DF) or transposed direct form (TDF) [29]. The transposed form and the direct form of a FIR filter are equivalent. It's easy to prove that, in direct form, the word length of each delay element is equal to the word length of the input signal. However, in the transposed form, each delay element has a longer word length than that in the direct form. The transposed structure reduces the critical path delay, but it uses more hardware. DF FIR filter is area-efficient, while the TDF filter is delay-efficient. In this paper, the architecture of the proposed FIR filter is presented. It is based on the transposed direct form FIR filter structure as shown in Figure 1. This structure comprises adders, D flip-flops, and multipliers.

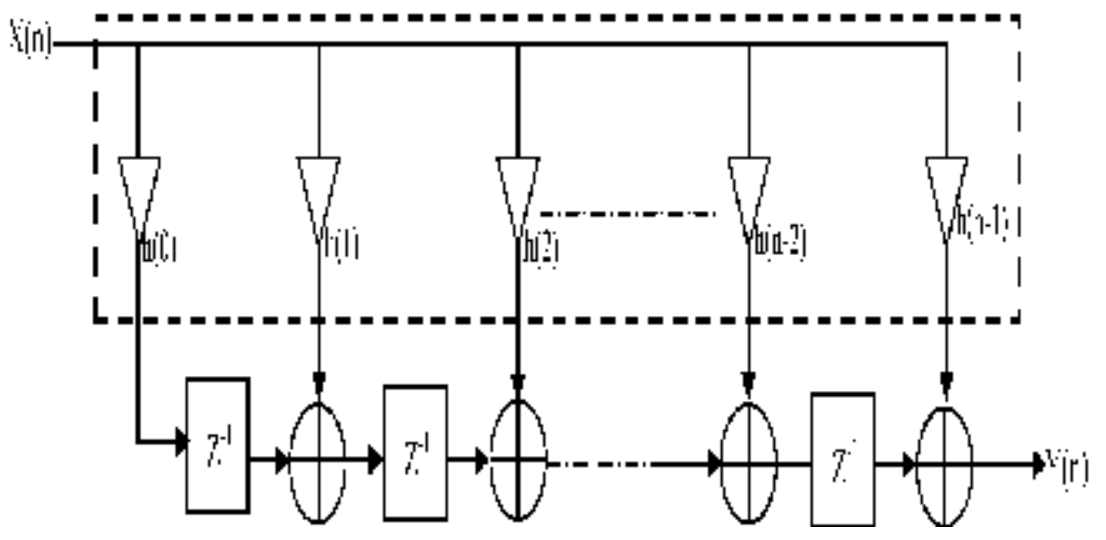

Figure 1.

Transposed direct form FIR filter architecture.

\section{QCA review}

The QCA approach, introduced in 1993 by Lent et al. [6], is able to replace devices based on field-effect transistor (FET) on nanoscale. This nanotechnology was conceived based on some of Landauer's ideas regarding energy-efficient and robust digital devices [30]. It consists of an array of cells. Each cell contains four quantum dots at the corner of a square which can hold a single electron per dot. Only two electrons diametrically opposite are injected into a cell due to Coulomb interaction [31]. Through Coulombic effects, two possible polarizations (labeled -1 and 1) can be shaped. These polarizations are represented by binary " 0 " and binary " 1 " as shown in Figure 2. Figure 3 shows the propagation of logic " 0 " and logic " 1 ," respectively, from 


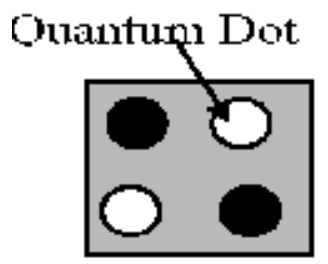

$13--1$

Binary " 0 "

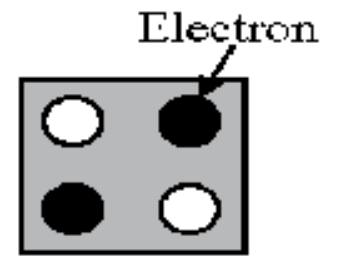

l' -1

Binary ' 1 '

Figure 2.

Two different polarizations of quantum-dot cell.

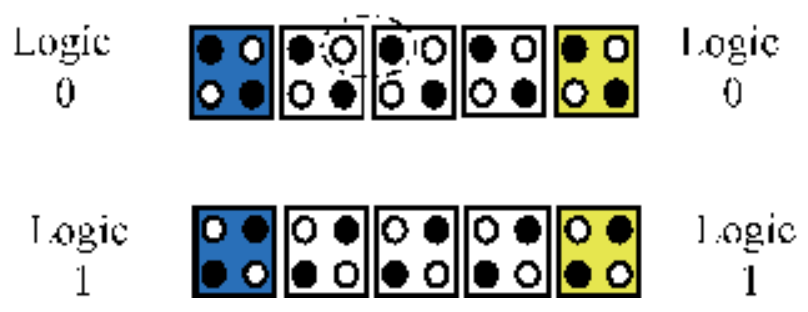

Figure 3 .

QCA binary wires.

i

\begin{tabular}{|c|c|c|c|}
\hline & $\begin{array}{ll}0 & 0 \\
0 & 0\end{array}$ & $\begin{array}{l}00 \\
00\end{array}$ & Clutpul \\
\hline 00 & 100 & & colog \\
\hline 00 & 100 & & colo \\
\hline & $\begin{array}{l}00 \\
00\end{array}$ & $\begin{array}{lll}0 & 0 \\
0 & 0\end{array}$ & \\
\hline
\end{tabular}

îi!

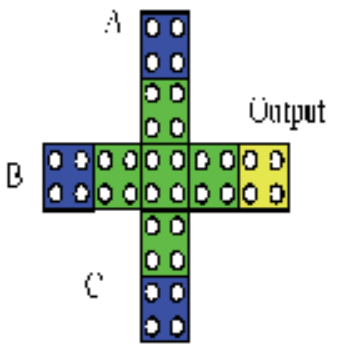

ibi

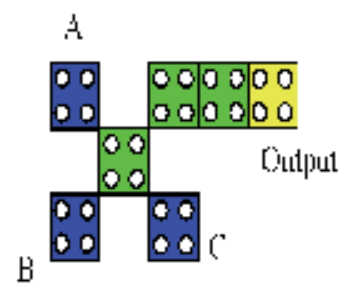

ici)

Figure 4 .

Standard gates: Inverter gate (a) and majority gate $(b, c)$.

input to the output in QCA binary wires due to the Coulombic repulsion. Generally, in neighboring cells, the coulombic interaction between electrons is used to implement many logic functions which are controlled by the clocking mechanism [32].

A majority and inverter gates are the fundamental logic gates in the QCA implementations which are composed of some QCA cells as shown in Figure $4[7,33]$. Furthermore, the majority gate acts as an AND gate and OR gate just by setting one input permanently to 0 or 1 . It has a logical function that can be expressed by Eq. (4):

$$
\mathrm{MV}(\mathrm{a}, \mathrm{b}, \mathrm{c})=\mathrm{AB}+\mathrm{BC}+\mathrm{AC}
$$

\subsection{QCA clocking}

The clocking system is an important factor for the dynamics of QCA. Its principal functions are the synchronization of data flows and the implementation of 
adiabatic cell operation which enables QCA circuits with high energy efficiency [34]. Generally, QCA clocking is presented with four different phases which are switch, hold, release and relax as illustrated in Figure 5. During the switch phase, which actual computations are occurred, the barriers are raised, and a cell is affected by the polarization of its adjacent cells, and a distinctive polarity is obtained. During the hold phase, the barriers are high, and the polarization of the cell is retained. During the release phase, the barriers are lowered, and the cell loses the polarity. During the relax phase, the cell is non-polarized [35].

Over recent years, various clocking schemes have been proposed, but they have introduced some difficulties such as long paths for feedbacks [35]. Recently, USE clocking scheme is a proposed technique for clocking and timing of the QCA circuits. It may be implemented using actual fabrication technologies of integrated circuits. This scheme can design feedback paths with different loop sizes, and its routing is flexible [20]. It defines a grid of clock zones, which are consecutively numbered from 1 to 4 as depicted in Figure 6 . This grid ensures the correct arrangement for the clock zones. Much information about the clocking circuitry are mentioned in [20].

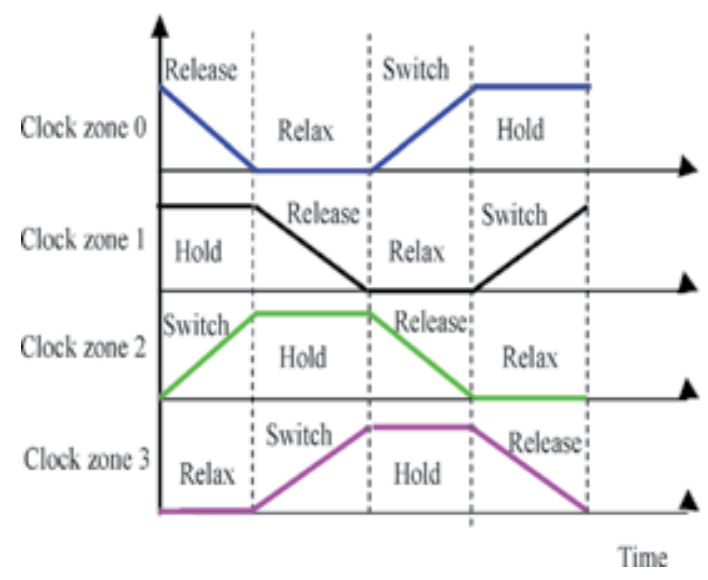

Figure 5 .

QCA clock zones.

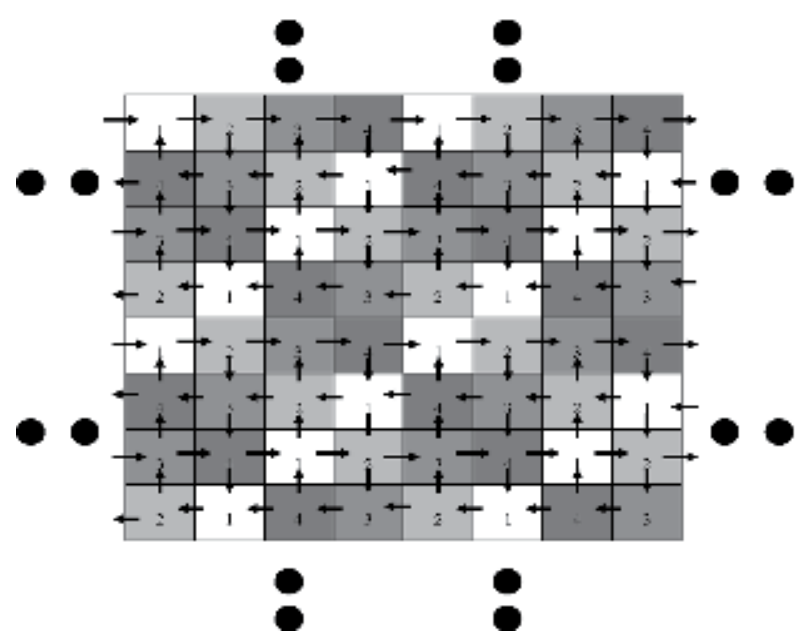

Figure 6.

QCA USE structure. 


\section{QCA FIR implementation}

The proposed QCA FIR filter consists of three principal components:

- A D flip-flop to implement a simple delay

- A multiplier to implement the coefficients

- An adder to sum the nodes at the end of each tap

\subsection{QCA D flip-flop}

Flip-flop is a circuit that may be used to store state information (" 0 " or " 1 " logic value). Here, the structure of the proposed D flip-flop is illustrated in Figure 7a which includes three majority gates and one inverter gate. The logic equation of the $\mathrm{D}$ flip-flop is represented by the following equation:

$$
Q_{(t)}=\operatorname{CLk} \cdot \mathrm{D}+\overline{\mathrm{CLk}} \cdot Q_{(t-1)}
$$

Figure 7b illustrates the proposed QCA flip-flop. It includes 79 cells with an area of $0.15 \mu \mathrm{m}^{2}$. It takes five clock periods for the inputs to reach the output, and first meaningful output comes on the sixth clock.

\subsection{QCA $4 \times 4$ multiplier}

Multiplier plays an important role in DSP systems. In divers' DSP application, it is not needed to utilize all output bits of multiplier. As in most of the FIR implementation, the FIR output can also be obtained using only the MSB bits of the multiplier output [29]. In literature, there are various algorithms of multiplier such as array multiplier, parallel multiplier and booth multiplier [36-39], which consumed more area and could not meet the criteria of propagation delay. This problem has been overcome in this paper by making use of Vedic multiplier which is much faster with minimum propagation delay [40-43]. To design the QCA circuit, we have used the version of the circuit proposed in [44]. Figure 8 demonstrates the schematic of 4-bit Vedic multiplier architecture where $A=A_{3} A_{2} \ldots A_{0}$ and $B=$ $B_{3} B_{2} \ldots B_{0}$ are the inputs and the outputs signal for the multiplication result are $P=$ $P_{7} P_{6} \ldots \ldots P_{0}$. The implementation of this multiplier can be done by using four $2 \times 2$ Vedic multiplier blocks and three 4-bit adder blocks.

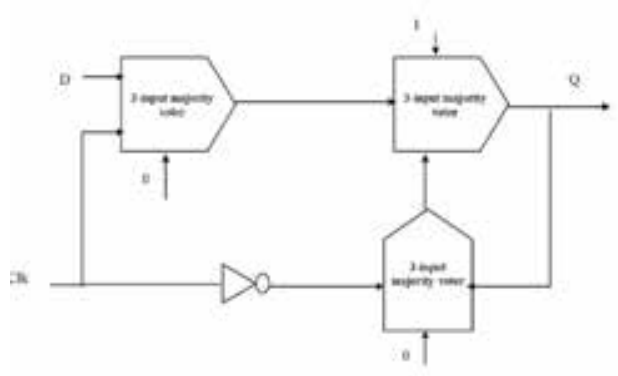

(a)

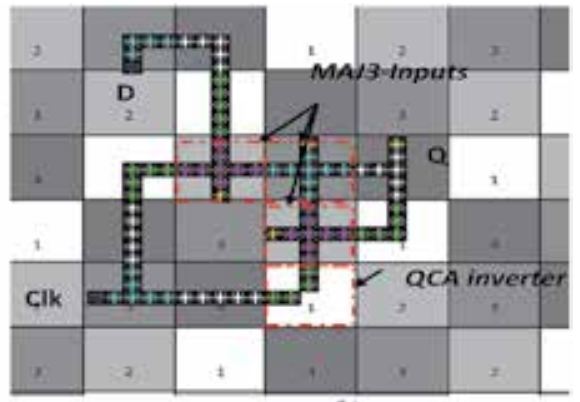

(b)

Figure 7.

Proposed (a) logical diagram and (b) QCA layout of D flip-flop. 
Design of 4-Bit 4-Tap FIR Filter Based on Quantum-Dot Cellular Automata (QCA) Technology... DOI: http://dx.doi.org/10.5772/intechopen.90193

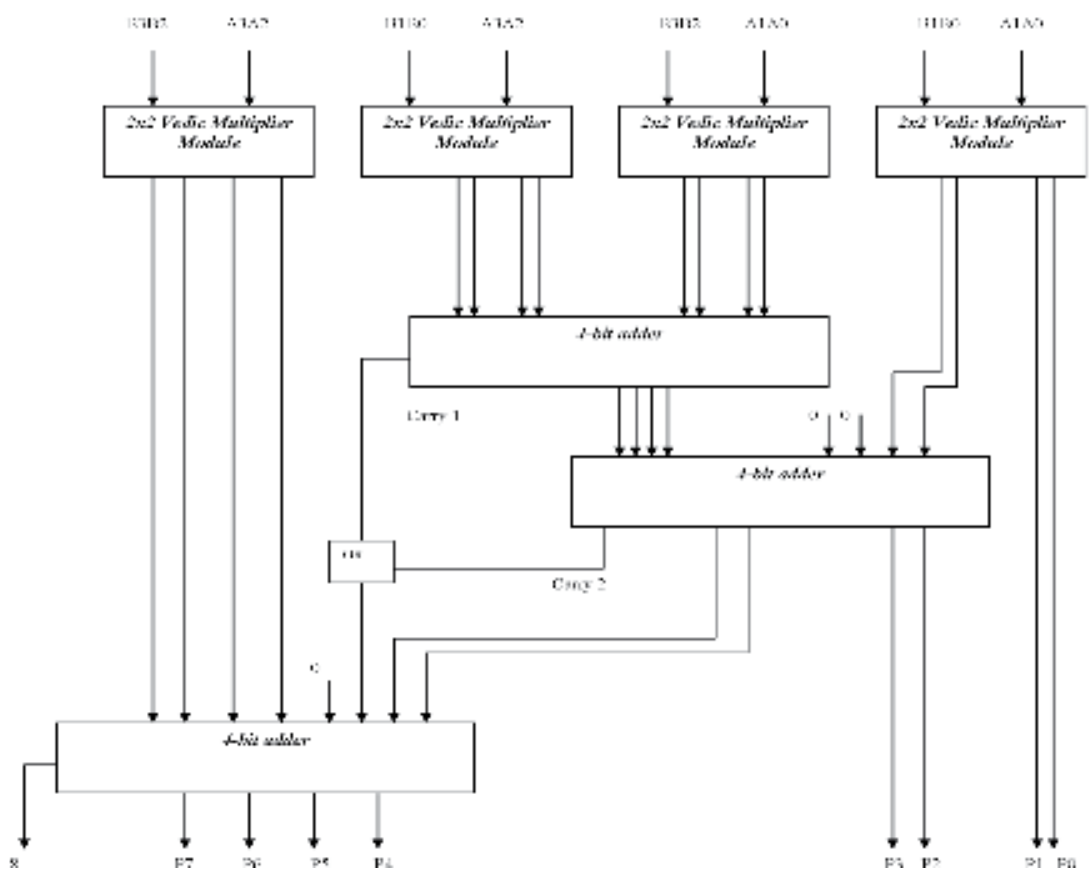

Figure 8.

Block diagram of 4-bit Vedic multiplier.

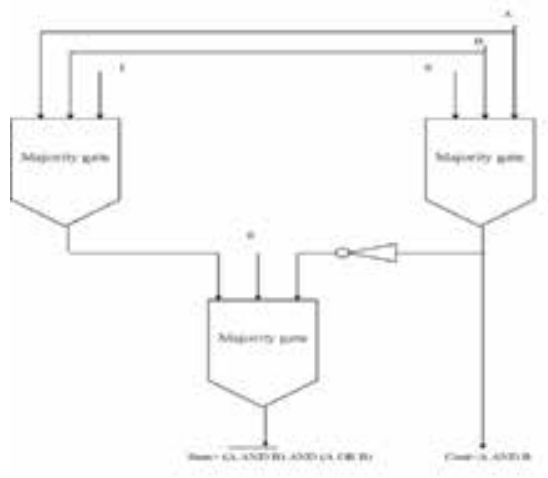

(a)

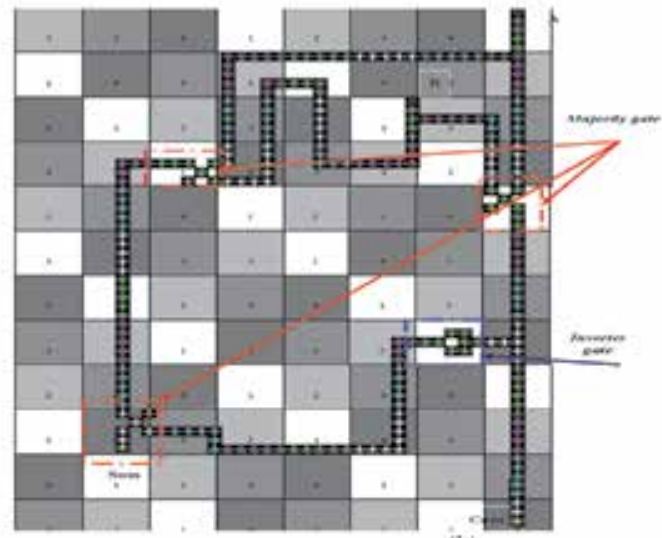

(b)

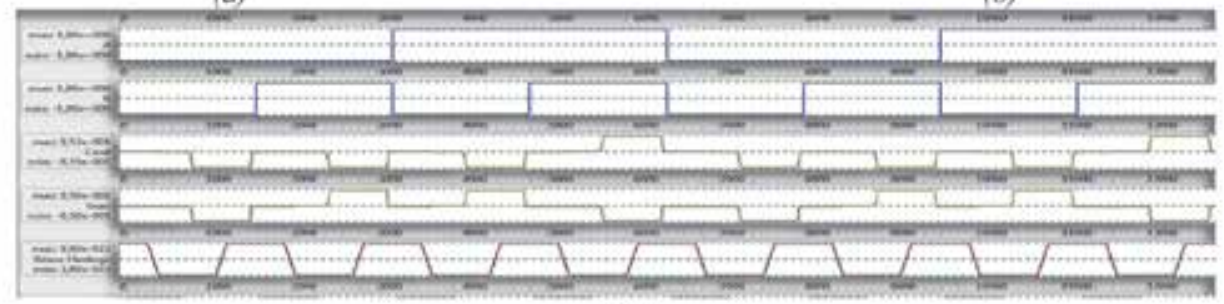

(c)

Figure 9.

Proposed (a) logical diagram, (b) QCA layout and (c) timing graph of half adder circuit.

\subsubsection{QCA 4-bit parallel adder}

The 4-bit adder performs computing function of the FIR filter. Therefore, the half and the full adder are used to construct the 4-bit binary adder. The proposed 


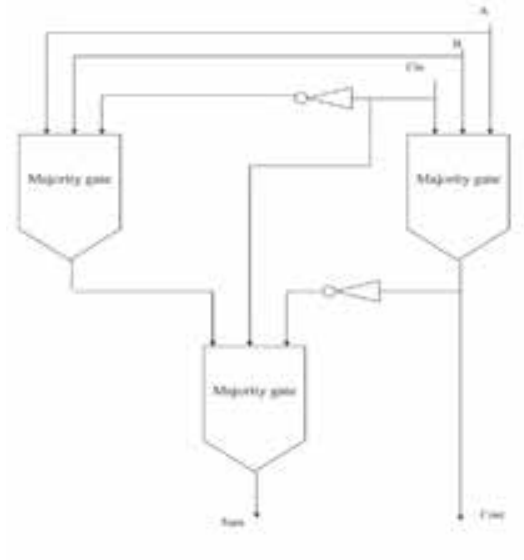

(a)

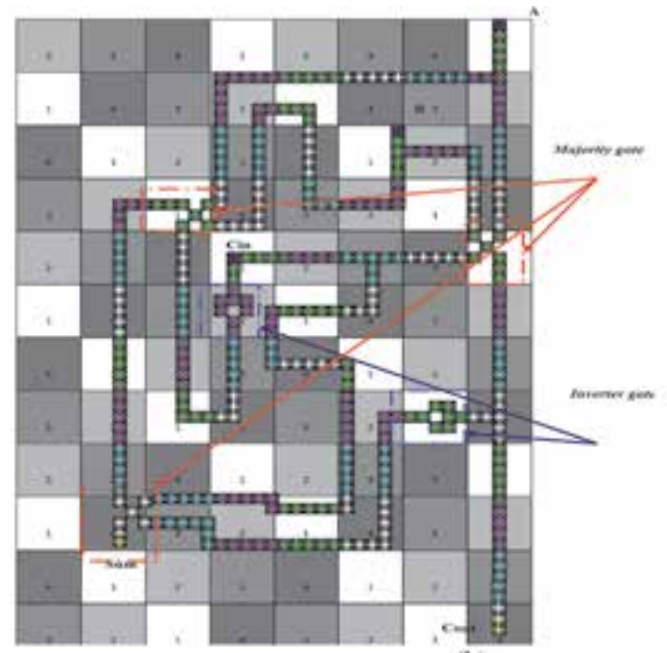

(b)

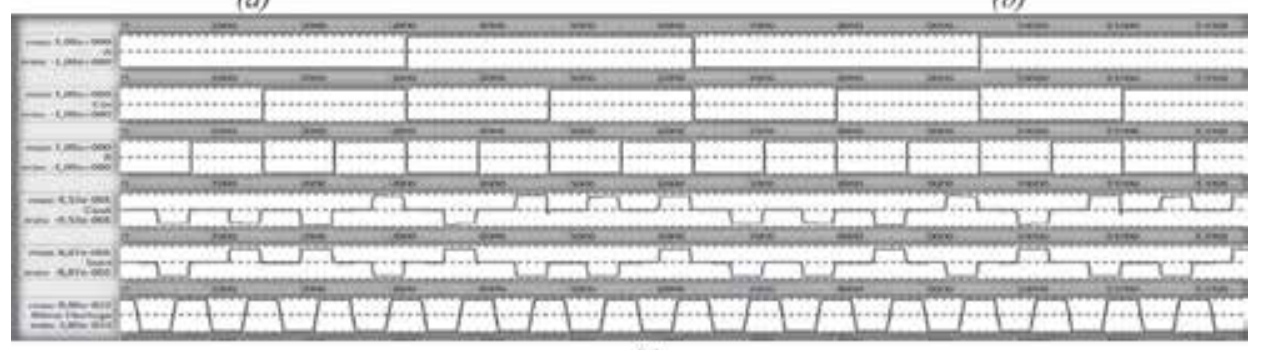

(c)

Figure 10.

Proposed (a) logical diagram, (b) QCA layouts and (c) simulation result of full adder circuit.

half adder is composed by three majority gates and one inverter gate. Figure 9 shows the block diagram and the QCA layout of the proposed half adder. It consists of 232 cells covering an area of $0.76 \mu \mathrm{m}^{2}$. It needs 16 clock phases to generate the sum and carry outputs. In addition, the proposed full adder consists of three majority gates and two inverters. Figure 10 depicts the block diagram and the QCA layout of the proposed full adder. For the proposed QCA full adder, the required number cells is 349 , and the required area is $0.76 \mu \mathrm{m}^{2}$. It requires 16 clock phases. The parallel adder layout in size of 4-bit is depicted in Figure 11. It is designed by cascading one-half adder and three 1-bit adders. In this way, the carry out (Cout) is then transmitted to the carry in (Cin) of the next higher-order bit. The final outcome creates a sum of 4 bits plus a carry out (Cout 4). This design uses 2735 cells in its structure. It consists of a circuit area of $11.46 \mu \mathrm{m}^{2}$. This circuit has a critical path length of 61 clock zones which is designated by a blue dashed line.

\subsubsection{QCA $2 \times 2$ vedic multiplier}

The block diagram of $2 \times 2$ bit Vedic multiplier is shown in Figure 12. Firstly, $\mathrm{B} 0$ is multiplied with A0; the generated partial product is considered as an LSB of final product.

Secondly, B0 is multiplied with A1,and B1 is multiplied with A0. To add the generated partial products $\left(\mathrm{B} 0^{*} \mathrm{~A} 1+\mathrm{A} 0^{*} \mathrm{~B} 1\right)$, a QCA half adder is required, which generates a 2-bit result (Carry and $\mathrm{S} 1$ ), in which $\mathrm{S} 1$ is considered as the second bit of the final product and Carry is saved as pre-carry for the next step. 


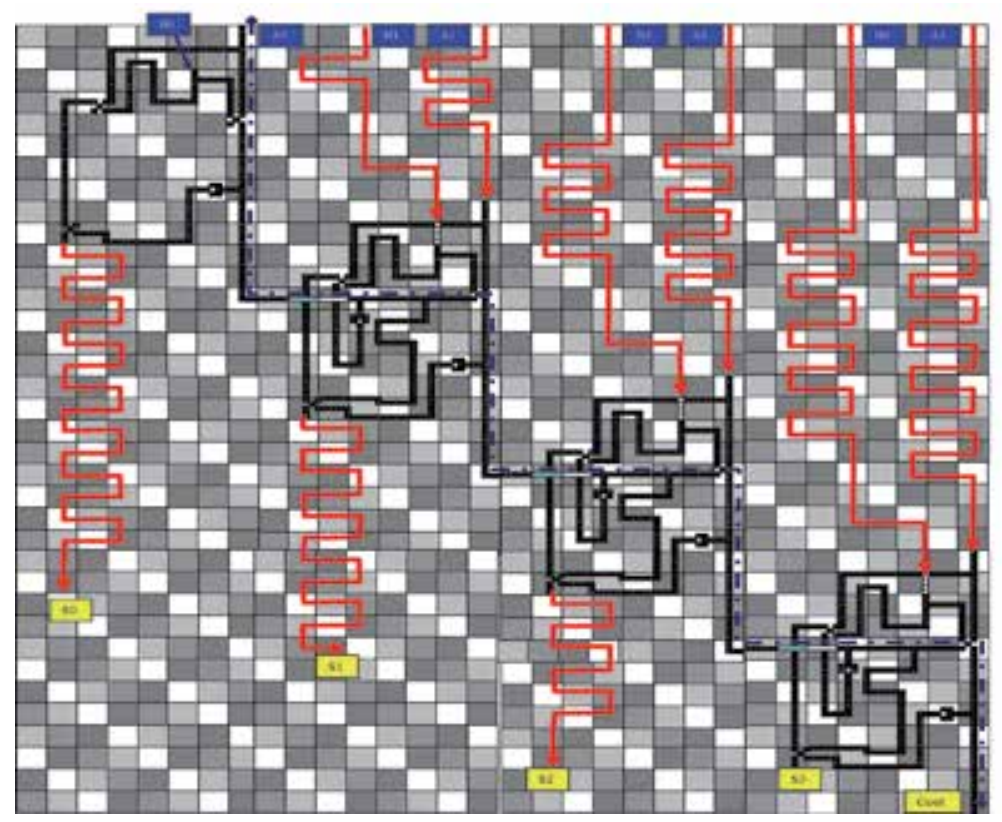

Figure 11.

Proposed QCA layouts of 4-bit parallel binary full adder.

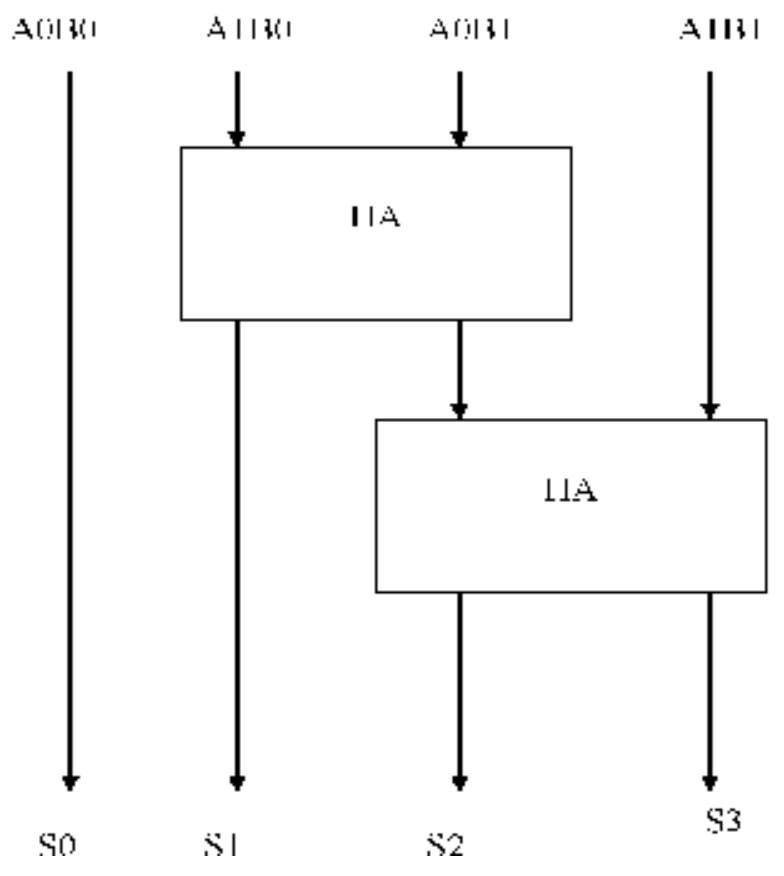

Figure 12.

$2 \times 2$ block diagram.

Finally, B1 is multiplied with A1, and the overall product term will be obtained for $2 \times 2$ Vedic multiplier. Here, four majority gates and two half adder circuits are used, and the output will be four bits (s0, s1, s2 and s3). 
The proposed $2 \times 2$ multiplier takes only 1683 QCA cells with a region of $8.42 \mu \mathrm{m}^{2}$.The simulated result of the proposed Vedic multiplier confirms that the expected operation is correctly achieved with 60 clock zones delay as depicted in Figure 13.

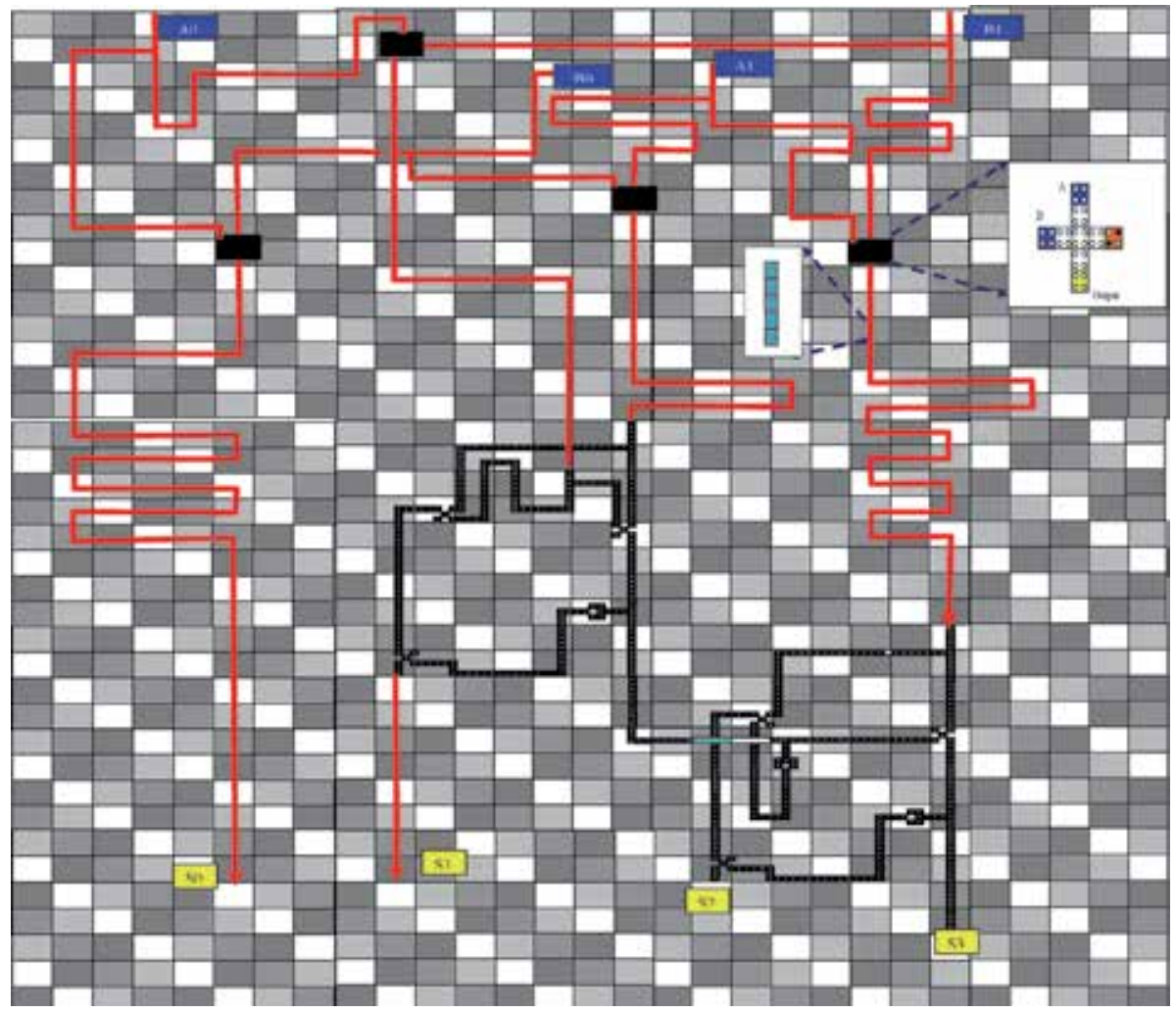

Figure 13.

The QCA implementation of $2 \times 2$ multiplier.

\begin{tabular}{lc}
\hline Parameter & Value \\
\hline Number of samples & 12,800 \\
\hline Convergence tolerance & 0.001000 \\
\hline Radius of effect & $65,000000(\mathrm{~nm})$ \\
\hline Relative permittivity & $12,900,000$ \\
\hline Clock low & $3800000 \mathrm{e}-023$ \\
\hline Clock high & $9800000 \mathrm{e}-022$ \\
\hline Clock shift & $0,000000 \mathrm{e}+000$ \\
\hline Clock amplitude factor & $2,000,000$ \\
\hline Layer separation & $11,500,000$ \\
\hline Maximum iterations per sample & 100 \\
\hline
\end{tabular}

Table 1.

Bistable approximation parameter model. 


\subsection{QCA adder}

Since the FIR output can be obtained using only the MSB bits of the Vedic multiplier output, for the proposed structure of FIR filter, we need a 4-bit QCA adder. The same 4-bit adder designed above is used in this subsection (Table 1).

\section{Results and discussions}

The complete QCA FIR design is implemented using the functional units discussed in the previous section. The implementation and the simulation of the proposed hardware designs are achieved by using QCADesigner 2.0.3 tool [45]. The coherence vector simulation engine is used for this purpose. Table 2 depicts the simulation parameters. In the first step, the sub-module schematic and layout is completed and verified by functional simulations.

These designs have been implemented using a free and a regular USE clock scheme. In addition, we have successfully demonstrated that sub-module design of FIR unit properly satisfies all logic and timing constraints by using the $4 \times 4$ USE grid with a square dimension of $5 \times 5$ QCA cells. In this direction, with a welldefined methodology and regular timing zones, this design is a standard candidate for fabrication. We note that our proposed entire system requires a huge number of QCA cells mostly due to the long wires necessary to delay compensation. Since the proposed FIR circuit based on QCA technology has started to bloom, we have only compared the full adder module with regular standard scheme circuits. Table 2 shows a comparison of the proposed full adder with some existing designs [35, 46]. The proposed full adder has 1.13, 56.9 and $11 \%$ improvements, respectively, in terms of cell count, area occupation and circuit latency as compared to that reported in [35].

In QCA technology, the power consumption of any circuit depends on the number of majority and inverter gates [47]. Therefore, this technology reduces more power than CMOS technology. The consumption of FIR unit in QCA-18 nm technology is valuing $1.6 \mathrm{~mW}$. This value is carried out using QCADesigner-E software [48].

However, the QCA FIR circuit requires $97.74 \%$ lesser power consumption than the previous existing designs [49]. In addition, the proposed design of FIR filter can operate at a higher frequency (upper than $1 \mathrm{GHz}$ ) than the conventional solution, and it can be useful for future digital signal processing applications for providing excellent processing speed. The overall performance of the proposed QCA design is therefore superior to the existing techniques in terms of power consumption. In this way, we think that this work forms an essential step in the building of QCA circuits for low-power design in this area.

Influence of temperature variations on the polarization of the proposed design has also been investigated. Figure 14 illustrates the effect of polarization on output of FIR circuit due to temperature variations. QCADesigner tool is used to observe this effect. By increasing temperature the AOP of any output cell of the QCA circuit

\begin{tabular}{lccc}
\hline Design & Cell count & Area $\left(\boldsymbol{\mu m}^{2}\right)$ & Clock no. cycle \\
\hline Full adder [35] & 353 & 1.764 & 18 \\
Full adder [46] & 324 & 0.77 & 18 \\
Proposed full adder & 349 & 0.76 & 16 \\
\hline
\end{tabular}

Table 2.

Comparison of various full adders. 


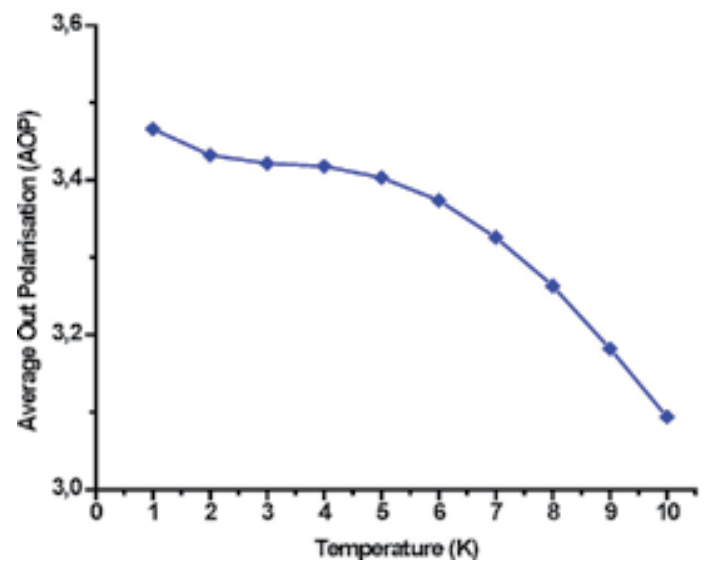

Figure 14.

Effect of polarization on output of FIR filter due to temperature.

is decreased. Therefore, between $1 \mathrm{~K}$ and $7 \mathrm{~K}$, the FIR circuit works efficiently. Over $7 \mathrm{~K}$, the circuit falls down radically and produces incompatible outputs.

\section{Conclusion}

Design of low-power high-speed FIR filter is always a challenge for DSP applications. In this article, a novel design of FIR filter architecture in the QCA technology has been presented. The functionality of the proposed circuits has been verified with QCADesigner version 2.0.3 software. The proposed QCA FIR achieves up to $1 \mathrm{GHz}$ frequency and consumes $1.6 \mathrm{~mW}$ power. By comparison of previous designs and the proposed design, it could be concluded that the proposed design has appropriate features and performance. Therefore, this work will provide better silicon area utilization, maximization of clock speed and very low-power consumption than traditional VLSI technology. It should be an important step towards highperformance and low-power design in this field. Future extensions, such as various applications based on this QCA FIR unit, could be investigated.

\section{Author details}

Ismail Gassoumi $^{1 *}$, Lamjed Touil ${ }^{1}$, Bouraoui Ouni $^{2}$ and Abdellatif Mtibaa ${ }^{1}$

1 Laboratory of Electronics and Microelectronics, University of Monastir, Tunisia

2 Networked Objects Control and Communication Systems Laboratory, University of Sousse, Tunisia

*Address all correspondence to: ismail.gassoumi@fsm.rnu.tn

\section{IntechOpen}

(C) 2019 The Author(s). Licensee IntechOpen. This chapter is distributed under the terms of the Creative Commons Attribution License (http://creativecommons.org/licenses/ by/3.0), which permits unrestricted use, distribution, and reproduction in any medium, provided the original work is properly cited. (cc) BY 


\section{References}

[1] Dennard RH, Gaensslen FH, Yu HN, Rideovt VL, Bassous E, Leblanc AR. Design of ion-implanted MOSFET's with very small physical dimensions. IEEE Solid-State Circuits Society Newsletter. 2007;12(1):38-50

[2] Bernstein K, Cavin RK, Porod W, Seabaugh A, Welser J. Device and architecture outlook for beyond CMOS switches. Proceedings of the IEEE. 2010; 98(12):2169-2184

[3] Bondy PK. Moore's law governs the silicon revolution. Proceedings of the IEEE. 2002;88(1):78-81

[4] Haron NZ, Hamdioui S. Why is CMOS scaling coming to an END? In: 3rd IEEE International Design and Test Workshop (IDT); 2008, pp. 98-103

[5] International Technology Roadmap for Semiconductors, Process Integration Devices and Structures (PIDS). Available from: http://www.itrs.net/Links/ 2011ITRS/Home2011.htm, 2011 Edition

[6] Lent CS, Tougaw PD, Porod W, Bernstein GH. Quantum cellular automata. Nanotechnology. 1993;4: 49-57

[7] Angizi S, Sarmadi S, Sayedsalehi S, Navi K. Design and evaluation of new majority gate-based RAM cell in quantum-dot cellular automata. Microelectronics Journal. 2015;46:43-51

[8] Azghadi MR, Kavehie O, Navi K. A novel design for quantum-dot cellular automata cells and full adders. arXiv preprint arXiv:1204.2048; 2012

[9] Berzon D, Fountain TJ. A memory design in QCAs using the SQUARES formalism. In: Great Lakes Symposium on VLSI. 1999. pp. 166-169

[10] Walus K, Vetteth A, Jullien G, Dimitrov V. Ram design using quantum-dot cellular automata. In:

Technical Proceedings of the

Nanotechnology Conference and Trade Show, vol. 2. 2003. p. 160163

[11] Lamjed T, Ismail G, Radhouane L, Bouraoui O. Efficient design of BinDCT in quantum-dot cellular automata (QCA) technology. IET Image

Processing. 2018;12(6):1020-1030

[12] Kim SW, Swartzlander EE. Parallel multipliers for quantum-dot cellular automata. In: Nanotechnology Materials and Devices Conference; Traverse City. 2009. pp. 68-72

[13] Kim SW, Swartzlander EE.

Multipliers with coplanar crossings for quantum-dot cellular automata. In: Conference on Nanotechnology; Seoul. 2010. pp. 953-957

[14] Balali M, Rezai A, Balali H, Rabiei F, Emadi S. Towards coplanar quantumdot cellular automata adders based on efficient three-input XOR gate. Results in Physics. 2017;7:1989-1995

[15] Sasamal TN, Singh AK, Mohan A. Efficient design of reversible alu in quantum-dot cellular automata. Int J Light Electron Optics. 2016;15: 6172-6182

[16] Sasamal TN, Singh AK, Ghanekar U. Design of non-restoring binary array divider in majority logic-based QCA. Electronics Letters. 2016;52(24): 2001-2003

[17] Kianpour M, Nadooshan RS. A novel modular decoder implementation in quantum-dot cellular automata (QCA). In: International Conference on Nanoscience Technology and Societal Implications (NSTSI). 2011. pp. 1-5

[18] Kianpour M, Nadooshan RS. A novel quantum dot cellular automata X-bit $\mathrm{x}$ 32-bit SRAM. In: IEEE Transactions on 
Very Large Scale Integration (VLSI) Systems; March 2016. pp. 827-836

[19] Chougule PP, Sen B, Dongale TD. Realization of processing in-memory computing architecture using quantum dot cellular automata. Microprocessors and Microsystems. 2017;2:49-58

[20] Campos C, Marciano A, Vilela OP, Sill Torres F. Use: A universal, scalable, and efficient clocking scheme for QCA. IEEE Transactions on Computer-Aided Design of Integrated Circuits and Systems. 2016;35(3):513-517

[21] Mahesh R, Vinod AP. New reconfigurable architectures for implementing FIR filters with low complexity. IEEE Transactions on Computer-Aided Design of Integrated Circuits and Systems. 2010;29(2): 275-288

[22] Park SY, Meher PK. Efficient FPGA and ASIC realizations of a DA-based reconfigurable FIR digital filter. IEEE Transactions on Circuits and Systems II: Express Briefs. 2014;61(7):511-515

[23] Gustafsson O. A difference based adder graph heuristic for multiple constant multiplication problem. In: Proceedings of the IEEE International Symposium on Circuits Systems. 2007. pp. 1097-1100

[24] Mahesh R, Vinod AP. A new common subexpression elimination algorithm for realizing low-complexity higher order digital filters. IEEE Transactions on Computer-Aided Design of Integrated Circuits and Systems. 2008;27(2):217-229

[25] Chitra E, Vigneswaran T. An efficient low power and high speed distributed arithmetic design for FIR filter. Indian Journal of Science and Technology. 2016;9(4):1-5

[26] Yu Z, Yu ML, Azadet K, Wilson AN. A low power FIR filter design technique using dynamic reduced signal representation. In: Proceedings of the International Symposium on VLSI Technology, Systems, and Applications. 2001. pp. 113-116

[27] Padmapriya S, Lakshmi VP. Design of an efficient dual mode reconfigurable FIR filter architecture in speech signal processing. Microprocessors and Microsystems. 2015;39:521-528

[28] Hong Y, Lian Y. A memristor-based continuous time digital FIR filter for biomedical signal processing. IEEE Transactions on Circuits and Systems I: Regular Papers. 2015;62(5):1392-1401

[29] Hsiao SF, Jian ZH, Chen MC. Lowcost FIR filter designs based on faithfully rounded truncated multiple constant multiplication/accumulation. IEEE Transactions on Circuits and Systems II: Express Briefs. 2013;60(5): 287-291

[30] Lent CS, Snider GL. The development of quantum-dot cellular automata. In: Anderson NG, Bhanja S, editors. Field-Coupled Nanocomputing: Paradigms, Progress, and Perspectives. Berlin, Heidelberg: Springer Berlin Heidelberg; 2014. pp. 3-20

[31] Tougaw PD, Lent CS. Logical devices implemented using quantum cellular automata. Journal of Applied Physics. 1994;75:1818-1825

[32] Lent CS, Isaksen B. Clocked molecular quantum-dot cellular automata. IEEE Transactions on Electron Devices. 2003;50(9):1890-1896

[33] Momenzadeh M, Huang J, Lombardi F. Design and Test of Digital Circuits by Quantum-Dot Cellular Automata. Norwood, MA: USA. Artech House; 2008. pp. 37-67

[34] Lent CS, Tougaw PD. A device architecture for computing with 
quantum dots. Proceedings of the IEEE. 1997;85(4):541-557

[35] Vankamamidi V, Ottavi M, Lombardi F. Two dimensional schemes for clocking/timing of QCA circuits. IEEE Transactions on Computer-Aided Design of Integrated Circuits and Systems. 2008;27(1):34-44

[36] Manjunath KE, Harikiran V, Manikanta K. Design and implementation of $16 \times 16$ modified booth multiplier. In: IEEE (IC-GET). 2015

[37] Yang Z, Xiao J. The design and simulation of array multiplier improved with pipeline techniques. In: IEEE (EMEIT). 2011

[38] Darjn E et al. On the use of approximate adders in carry-save multiplier-accumulators. In: IEEE International Symposium on Circuits and Systems (ISCAS). 2017

[39] Kokila JB, Nithish KV, Pavithra S. Low power wallace tree multiplier using modified full adder. In: IEEE (ICSCN). 2015

[40] Tiwari HD, Ganzorig G, Chan MK. Multiplier design based on ancient Indian Vedic mathematics. In: International Conference on SoC Design. 2008. pp. 65-68

[41] Huddar SR, Rupanagudi SR, Kalpana M. Novel high speed Vedic mathematics multiplier using compressors. Kottayam, India: IEEE International multi Conference. 2013: 465-469

[42] Akhter S. VHDL implementation of fast $\mathrm{N} \times \mathrm{N}$ multiplier based on vedic mathematic. 2007 18th European Conference on Circuit Theory and Design. 2007. DOI: 10.1109/

ecctd.2007.4529635
[43] Verma P. Design of $4 \times 4$ bit vedic multiplier using EDA tool. International Journal of Computer Application. 2012; 48(20):32-35

[44] Kumar KS, Swathi M. 128-Bit Multiplier with Low-Area High-Speed Adder Based on Vedic Mathematics. Lecture Notes in Electrical Engineering. Singapore; 2017. pp. 163-172. DOI: 10.1007/978-981-10-4280-5_17

[45] Walus K, Dysart TJ, Jullien GA, Budiman RA. QCADesigner: A rapid design and simulation tool for quantumdot cellular automata. IEEE Transactions on Nanotechnology. 2004; 3:26-31

[46] Trindade A, Ferreira R, Nacif JAM, Sales D, Neto OPV. A Placement and routing algorithm for Quantum-dot Cellular Automata. 2016 29th Symposium on Integrated Circuits and Systems Design (SBCCI). 2016. DOI: 10.1109/sbcci.2016.7724048

[47] Liu W, Srivastava S, Lu L, et al. Are QCA cryptographic circuits resistant to power analysis attack? IEEE

Transactions on Nanotechnology. 2012; 11(6):1239-1251

[48] Frank ST, Robert W, Philipp N, Rolf D. An energy-aware model for the logic synthesis of quantum-dot cellular automata. IEEE Transactions on Computer-Aided Design of Integrated Circuits and Systems. 2018;37:1-11

[49] Krishnamoorthy R, Saravanan S. A novel flip-flop based error free, area efficient and low power pipeline architecture for finite impulse recursive system. Cluster Computing. 2018:22: 1-11. DOI: $10.1007 / \mathrm{s} 10586-018-2513-4$ 



\title{
DFT Study on Interaction of Estrone and Imidazolium-Based Hydrophobic Ionic Liquids
}

\author{
Sai Saravanan Ambi Venkataramanan and \\ Ramalingam Anantharaj
}

\begin{abstract}
Affinity of estrone on imidazolium-based hydrophobic ionic liquids was computed at the B3LYP/6-31G(d) level. Columbian interactions and other closed-shell interactions, in general, were observed to be pivotal to the binding of the EDC species on visualizing the optimized structures as well as probing the proximity of electronegative and electropositive groups between estrone and ionic liquids. The interaction strength was also studied using calculation of binding energy values of each system. [BMIM $]^{+}[\mathrm{PF} 6]^{-}$was found to be the most binding ionic liquid. Estrone was found to be highly bonded in the vicinity of [PF6 ${ }^{-}$species with a binding energy of $-9.57 \mathrm{kcal} / \mathrm{mol}$. The Ionic Liquids under study, $[\mathrm{BMIM}]^{+}[\mathrm{NTF} 2]^{-}$and $[\mathrm{BMIM}]^{+}[\mathrm{BF} 4]^{-}$, also illustrated promising binding nature with binding energies of -7.63 and $-8.61 \mathrm{kcal} / \mathrm{mol}$. AIM analysis was carried out to validate the nature of intermolecular interactions by calculating the topological properties at $(3,-1)$ bond critical points.
\end{abstract}

Keywords: DFT, endocrine disrupting compounds, computational quantum chemistry, molecular interaction

\section{Introduction}

Chemical substances that inhibit the reception sites in endocrine systems in our body are collectively termed as endocrine-disrupting compounds (EDCs). These carcinogens are proliferating in large quantities in all forms of water media, ranging from domestic water to ocean streams [1]. Although these contaminations are extremely minimal in quantity, such as in the order of $\mathrm{ppm}$, these prove to be a significant competitive inhibitor and obstruct the transmission of endocrine hormones to certain parts of our bodies leading to a diverse array of problems, including development of cancerous tissues and abnormal change in sexual orientation of a person altogether. Recent research works in the domain of wastewater treatment have shed some light on this issue stating that most wastewater discharge plants and sewage treatment plants currently being operated in the industry are also affected by exposure to EDCs [2].

Ionic liquids have been reported as novel green solvents in various domains ranging from catalysis [3] to extraction [4], owing to its ease in screening of these solvents by subtle manipulation of thermodynamic variables. A review publication by Tomasi 
et al. [5] presents a theoretical study of quantum mechanical continuum solvation models which is developed to overcome computational costs which attribute via explicit introduction of solvent molecules over the solute phase. Multiple articles have been presented using hydrophobic ionic liquids and DES for extraction of potential endocrine descriptors such as diethylstilbestrol, bisphenol-A, and dichlorodiphenyltrichloroethane (DDT) [6]. However, less emphasis is shed toward compounds such as estrone and other estrogen-based endocrine-disrupting compounds, in general.

$\mathrm{Ab}$ initio-based quantum chemistry methods attempt to solve the Schrödinger equation to extract intricate details such as electron distribution, underlying molecular interaction, as well as reactivity in a proposed virtual environment. Recent advancements in computational facilities have paved way to run these simulations in a much faster means and have also enabled theoretical chemists to solve a range of problems in disciplines ranging from spectroscopy [7] to solvent extraction [8]. In this work, we use ab initio calculations using benchmarked computational procedures to study the interacting behavior of estrone and ionic liquids such as $[\mathrm{BMIM}]^{+}[\mathrm{NTF} 2]^{-},[\mathrm{BMIM}]^{+}[\mathrm{PF} 6]^{-}$, and $[\mathrm{BMIM}]^{+}[\mathrm{BF} 4]^{-}$. This study is meant to be a primer for understanding the affinity of estrone so as to theoretically validate if the solvent is a potential extractor when commercially employed in standard liquidliquid extraction procedures.

\section{Computational section}

\subsection{Overview of computational quantum chemistry}

Density functional theory (DFT) is one of the most commonly employed methods to solve Schrödinger's equation by proposing a few justifiable approximations. In contradiction to the wave function methods used earlier, this method is the perfect adaptation for solving large systems involving a huge network of atoms and molecules. Since many rudimentary terms are overlapping in both the methods, the most primitive Hartree-Fock (HF) theory is used to explain the terms in a more elegant manner.

The energy computed from this method will take into account the potential energy of electrons and nuclei based on the relative positions with respect to each other as well as the average kinetic energy of electrons in every orbital. Assuming Born-Oppenheimer approximation, the relative velocity of nuclei is considered insignificant and so does its contribution in the kinetic energy of the system. The columbic interaction of electrons with respect to each other is brought about by assuming a mean field wherein each electron interacts with the averaged field of all the other electrons.

Kohn and Sham's work, in particular, attracted many researchers working in the field of DFT as they attempted to use the standard self-consistent field method to obtain the exchange-correlation energies as well apart from the basic Columbian energies obtained from HF theory [9]. Therefore, owing to their contribution, DFT is also colloquially referred to as Kohn-Sham (KS) formalism. Perdew et al. [10] proposed the advancements in the field of DFT functionals to that of a ladder, where every step leads to a better approximation and, hence, accuracy. The drawback of DFT method is that it can be used only for ground state computations as well as does not, by itself, reveal a detailed picture of electronic distribution owing to which, the optimized structure may not show any key resemblance when compared with other higher accurate methods. However, HF assumes linear combination of atomic orbital ( $\mathrm{LCAO}$ ) which helps to elucidate primitive picture 
of electrons in the molecule and, hence, can be used as an input for the method of higher accuracy, such as the configuration interaction method, which are collectively termed as post-HF methods.

DFT methods used in the present scenario describe the changes in total energy of the system, via exchange and correlation of electrons and electrons and nuclei using hybrid functionals. These sets of mathematical functions utilize a subset of exact exchange from HF, while the remaining uses predefined functions from each individual pure functional [11]. The most commonly used hybrid functional, obtained as a result of this implementation, is the B3LYP hybrid functional. As for the basis is concerned, standard 6-31G(d) basis set is invoked though higher accurate ECP sets are available [12] simply due to the fact that various literature studies in ionic liquids akin to that of ours have obtained better results using this model chemistry in conjunction with B3LYP.

\subsection{Computational details and methodology}

The interacting monomers, namely, estrone and each of the three ionic liquids $[\mathrm{BMIM}]^{+}[\mathrm{NTF} 2]^{-},[\mathrm{BMIM}]^{+}[\mathrm{PF} 6]^{-}$, and $[\mathrm{BMIM}]^{+}[\mathrm{BF} 4]^{-}$, were constructed using Gaussview6 [13] and were optimized separately at the B3LYP/6-31G(d) level using Gaussian16 program [14] to find the corresponding geometry and energy at the ground state. Since all the bonding parameters of the monomers are unknown, none of them were restricted under optimization.

The estrone is then introduced selectively to various regions in the vicinity of the ionic liquid such as near the electropositive and electronegative moieties, the imidazolium ring, and the cation and anion groups of ionic liquid to search for all possible interaction sites of the EDC. All these optimized systems can be visualized as various minima in the potential energy surface (PES), and only the properties and structure corresponding to the global minimum are presented for discussion.

The absence of imaginary frequencies at all optimized ground states justified that the proposed geometry is a minimum in the PES and not a transition state (TS) intermediate.

The keyword "output = wfn" is invoked along with the default settings to create. wfn extension file which is then used in multiwfn package to calculate topological properties at all bond critical points.

\section{Results and discussion}

\subsection{Optimized structures of the complex at the B3LYP/6-31G(d) level}

Optimized structures of estrone/ionic liquid complexes are depicted in Figure 1. Estrone was found closer to the anion species, especially just near the electronegative atoms having most negative partial charges in all the three cases. For instance, in the case of $[\mathrm{BMIM}]^{+}[\mathrm{NTF} 2]^{-}$and estrone, apart from $\mathrm{O}-\mathrm{H}$ and $\mathrm{F}-\mathrm{H}$ interactions which are visible from the proximity of the groups at the optimized structure, it can be seen that there is a substantial difference in the partial atomic charges of $\mathrm{S}(1.125 \mathrm{e})$ and $\mathrm{C}(-0.394 \mathrm{e})$ at one end followed by partial atomic charges $0.6 \mathrm{e}$ and -0.49 e on the carbon-bearing fluoride and hydrogen atoms on [NTF2] ${ }^{-}$and estrone, respectively. While in the other case such as $[\mathrm{BMIM}]^{+}[\mathrm{BF} 4]^{-}$and estrone, the vicinity of the bromine and fluoride is not justified by oppositely polarized moieties in estrone, however, also not indicative of an open-shell or covalent interaction as well simply because all the outer shell electrons of the atoms involved 

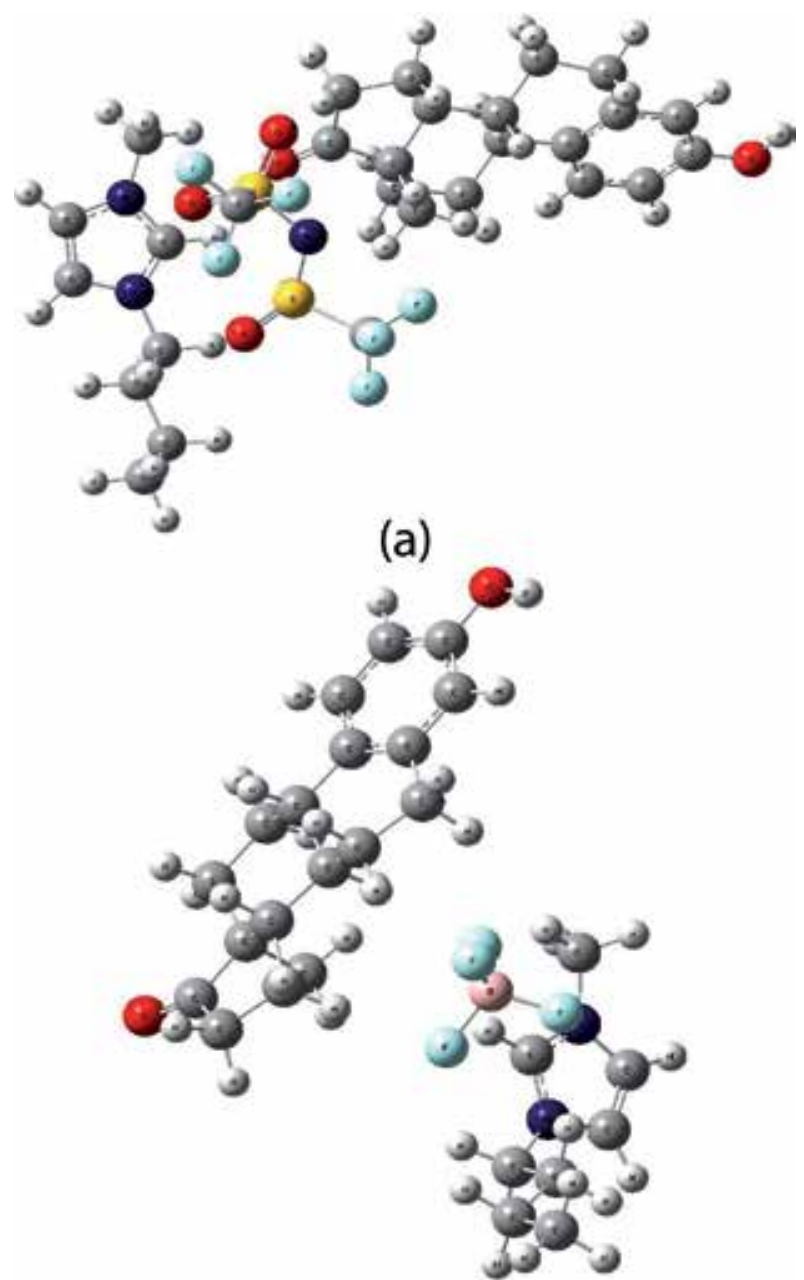

(b)

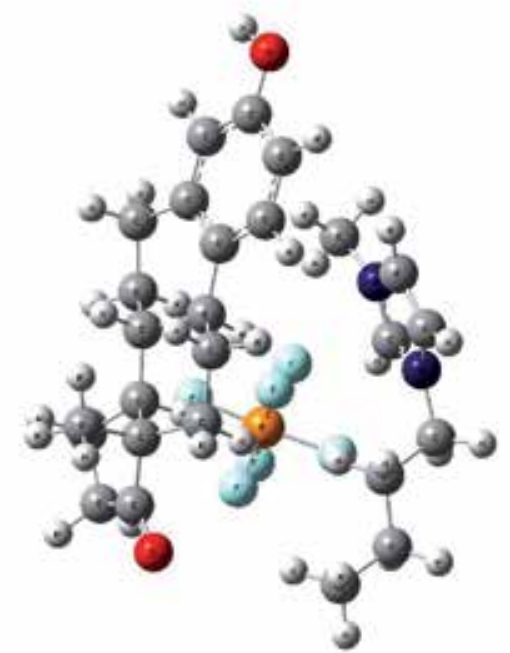

(c)

Figure 1.

Optimized structures of $[\mathrm{BMIM}]^{+}\left[\mathrm{NTF}_{2}\right]^{-}$and estrone (a), $[\mathrm{BMIM}]^{+}\left[\mathrm{BF}_{4}\right]^{-}$and estrone $(b)$, and $[B M I M]^{+}[P F 6]^{-}$and estrone (c) complexes at the B3LYP/6-31G(d) level. 
are already satisfied while, at the same time, not providing any window of opportunity for extending the coordination due to the absence of d-type orbitals in their individual atomic structure. These results suggest that electrostatic interactions and other closed-shell interactions between atoms of complimentary polarities are predominant when compared to the effects of covalent interactions and $\pi$ cloud effects contributed via imidazolium ring.

\subsection{Interaction energy calculations}

The schematic representation for complex formation is given by Eq. (1):

$$
A+B \rightarrow A \cdots B
$$

in which $\mathrm{A}, \mathrm{B}$, and $\mathrm{A} \ldots \mathrm{B}$ represent the estrone, ionic liquid, and estrone/ionic liquid complex, respectively.

Interaction energy calculations give a numerical estimate of the ability of the EDC to interact with the ionic liquid by means of chemisorptions occurring at the molecular level. These values are determined by Eq. (2):

$$
\Delta E_{\text {int }}=E_{A \cdots B}-\left[E_{A}+E_{B}\right]
$$

These interaction energy values are tabulated in Table 1 . It is to be noted that the values presented here include the zero-point correction.

Negative values indicate good interaction behavior of estrone in the presence of the ionic liquid. In comparison, it can be seen that $[\mathrm{BMIM}]^{+}[\mathrm{PF} 6]^{-}$and estrone system bind the complex with stronger interaction strength contributing to the

\begin{tabular}{|c|c|c|c|c|}
\hline \multicolumn{5}{|c|}{ Model chemistry: B3LYP/6-31G* } \\
\hline \multirow[t]{2}{*}{ Molecule/system } & \multirow{2}{*}{$\begin{array}{c}\text { Total electronic } \\
\text { energy (Hartrees) }\end{array}$} & \multirow[t]{2}{*}{ System } & \multicolumn{2}{|c|}{ Interaction energy } \\
\hline & & & (Hartrees) & (kcal/mol) \\
\hline$[\mathrm{BMIM}]^{+}[\mathrm{NTF} 2]^{-}$ & -2250.231194 & $\begin{array}{l}{[\mathrm{BMIM}]^{+}[\mathrm{NTF} 2]^{-}} \\
\text {and estrone }\end{array}$ & -0.012152 & -7.63 \\
\hline Estrone & -849.256955 & $\begin{array}{c}{[\mathrm{BMIM}]^{+}[\mathrm{PF} 6]^{-} \text {and }} \\
\text { estrone }\end{array}$ & -0.015246 & -9.57 \\
\hline$[\mathrm{BMIM}]^{+}[\mathrm{PF} 6]^{-}$ & -1363.714993 & $\begin{array}{c}{[\mathrm{BMIM}]^{+}[\mathrm{BF} 4]^{-} \text {and }} \\
\text { estrone }\end{array}$ & -0.013718 & -8.61 \\
\hline $\begin{array}{l}{[\mathrm{BMIM}]^{+}[\mathrm{NTF} 2]^{-}} \\
\text {and estrone }\end{array}$ & -3099.500301 & & & \\
\hline $\begin{array}{l}{[\mathrm{BMIM}]^{+}[\mathrm{PF} 6]^{-} \text {and }} \\
\text { estrone }\end{array}$ & -2212.987194 & & & \\
\hline$[\mathrm{BMIM}]^{+}[\mathrm{BF} 4]^{-}$ & -847.587891 & & & \\
\hline $\begin{array}{l}{[\mathrm{BMIM}]^{+}[\mathrm{BF} 4]^{-} \text {and }} \\
\text { estrone }\end{array}$ & -1696.858564 & & & \\
\hline
\end{tabular}
highest negative binding energy of $-9.57 \mathrm{kcal} / \mathrm{mol}$.

Total electronic energy of various species in Hartrees is also provided for reference, all results obtained at the $B 3 L Y P / 6-31 G(d)$ level.

${ }^{*}$ Denotes addition of $(d)$ polarizable function to the 6-31G Basis set.

Table 1.

Interaction energy of various estrone/ionic liquid systems in Hartrees and kcal/mol. 


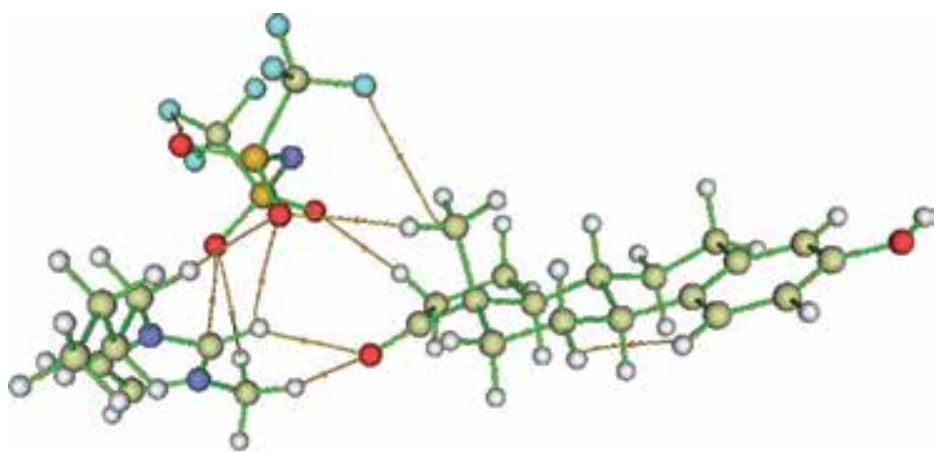

(a)

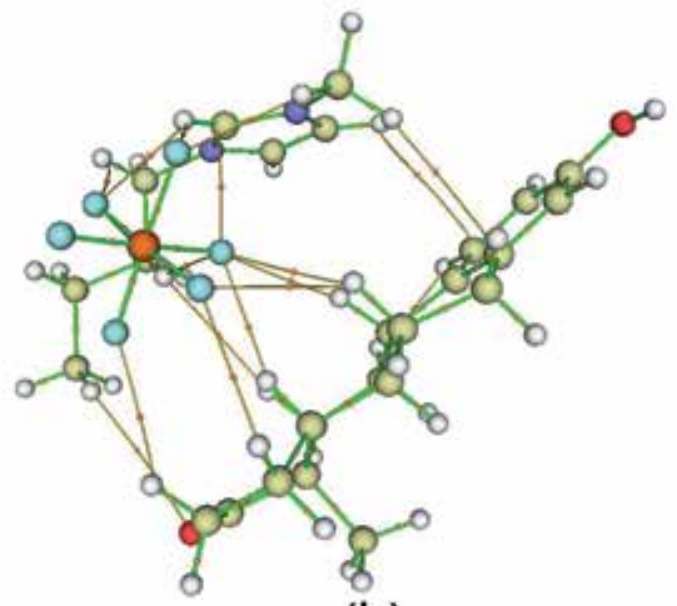

(b)

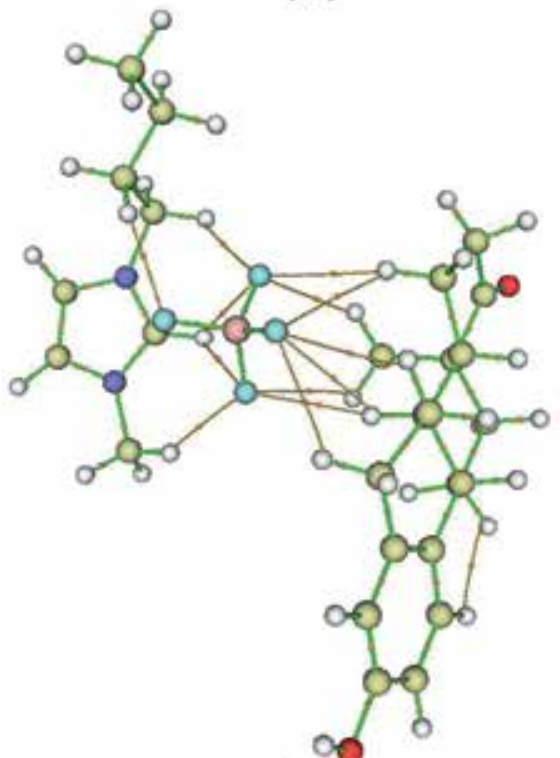

(c)

Figure 2.

Optimized structures of $[\mathrm{BMIM}]^{+}\left[\mathrm{NTF}_{2}\right]^{-}$and estrone (a), $[\mathrm{BMIM}]^{+}[\mathrm{PF} 6]^{-}$and estrone (b), and $[B M I M]^{+}\left[B F_{4}\right]^{-}$and estrone $(c)$ complexes at the $B 3 L Y P / 6-31 G(d)$ level containing $(3,-1)$ bond critical points (in orange). Color code: white, hydrogen; red, oxygen; yellow, carbon; royal blue, nitrogen; light blue, fluorine; pink, boron; brown, phosphorous; and dark yellow, sulfur; orange, $(3,-1)$ bond critical point. 


\subsection{Atoms in molecule (AIM) analysis}

$(3,-1)$ Bond critical points (BCPs) obtained from atoms in molecule (AIM) analysis are presented as the representation index of the electronic interaction and distribution between a given bond pair in this study. Multiwfn software is used to calculate the topological properties of bond critical points and perform complete AIM analysis [9]. Figure 2 gives a visual representation of $(3,-1)$ BCPs of estrone/ ionic liquid complexes. Previous literature studies proposed that the non-covalent interacting nature can alternatively be described by examining the ratio of Lagrangian kinetic energy $\left(G\left(r_{c}\right)\right)$ and potential energy density $\left(V\left(r_{c}\right)\right)$ given by $\left[-G\left(r_{c}\right) / V\left(r_{c}\right)\right][15]$. The aforementioned ratio at $(3,-1)$ bond critical points is used to determine the nature of interactions.

This topological study is used as a validation tool to justify the proximity of estrone in various cases. Firstly, on introduction of [BMIM $]^{+}[\mathrm{NTF} 2]^{-}$, we can see multiple hydrogen-bonded interactions with $\mathrm{O}$ and $\mathrm{F}$ atoms, evident with the discussion provided in the first section. Since these are hydrogen-bonded interactions and, hence, clearly non-covalent, other interactions such as the $\mathrm{C}-\mathrm{F}$ interaction between estrone carbon and $\mathrm{F}$ atom on $[\mathrm{NTF} 2]^{-}$are investigated. At this intermolecular critical point, we have $G\left(r_{c}\right)$ and $V\left(r_{c}\right)$ values of 0.003604 and -0.002431 , respectively, clearly having a ratio greater than 1 . In the second case, pertaining to $[\mathrm{BMIM}]^{+}[\mathrm{PF} 6]^{-}$, akin to the first case, most interactions are hydrogen-bonded interactions apart from the $\mathrm{C}-\mathrm{H}$ interactions between the cation of the ionic liquid and hydrogen of the EDC. The two critical points obtained bear $\mathrm{G}\left(\mathrm{r}_{\mathrm{c}}\right)$ and $\mathrm{V}\left(\mathrm{r}_{\mathrm{c}}\right)$ values of $0.002434,0.002517$ and $-0.001707,-0.001741$, respectively. In the case of $[\mathrm{BMIM}]^{+}[\mathrm{BF} 4]^{-}$, all intermolecular interactions are F-bonded interactions which simply arise out of difference in electronegativities and, hence, are completely noncovalent interactions.

Moreover, all interactions were found to have positive Laplacian of electron density at the intermolecular critical points (in the range of $0.01 \AA^{-5}$ ), which suggests closed-shell interaction nature. All the three cases reveal that intermolecular interactions are completely non-covalent in nature in conjunction with the observation made on assessing the optimized structure.

\section{Conclusions}

The interaction behavior of (1:1) estrone and imidazolium-based hydrophobic ionic liquids such as $[\mathrm{BMIM}]^{+}[\mathrm{NTF} 2]^{-},[\mathrm{BMIM}]^{+}[\mathrm{PF} 6]^{-}$, and $[\mathrm{BMIM}]^{+}[\mathrm{BF} 4]^{-}$ was studied at the B3LYP/6-31G(d) level of theory. The optimized structures were presented to study the vicinity of EDC with respect to each ionic liquid. The ZPE-corrected binding energy values were found to be negative, indicating fruitful interaction of the EDC and ionic liquid species. Based on the interaction strength, the affinity of estrone on ionic liquids can be described as [BMI $\mathrm{M}]^{+}[\mathrm{PF} 6]^{-}>[\mathrm{BMIM}]^{+}[\mathrm{BF} 4]^{-}>[\mathrm{BMIM}]^{+}[\mathrm{NTF} 2]^{-}$. AIM analysis was carried out to find the nature of interactions, so as to get the electronic distribution at the intermolecular region. It was found out that all interactions were characterized by positive Laplacian of electron density and $-G\left(r_{c}\right) / V\left(r_{c}\right)>1$ at intermolecular critical points illuminating the non-covalent nature of interactions existing between EDC and ionic liquids. 


\section{Author details}

Sai Saravanan Ambi Venkataramanan and Ramalingam Anantharaj*

Department of Chemical Engineering, SSN College of Engineering, Chennai, India

*Address all correspondence to: anantharajr@ssn.edu.in

\section{IntechOpen}

(C) 2019 The Author(s). Licensee IntechOpen. This chapter is distributed under the terms of the Creative Commons Attribution License (http://creativecommons.org/licenses/ by/3.0), which permits unrestricted use, distribution, and reproduction in any medium, provided the original work is properly cited. (cc) BY 


\section{References}

[1] Colborn T, VomSaal FS, Soto AM. Developmental effects of endocrine disrupting chemicals in wildlife and humans. Environmental Health Perspectives. 1993;101:378-398

[2] Westerhoff P, Yoon Y, Snyder S, Wert E. Fate of endocrine-disruptor, pharmaceutical, and personal care product chemicals during simulated drinking water treatment processes. Environmental Science \& Technology. 2005;39:6649-6671

[3] Welton T. Room-temperature ionic liquids. Solvents for synthesis and catalysis. Chemical Reviews. 1999;99:2071-2099

[4] Zhao W, Han M, Dai S. The extraction of polycyclic aromatic hydrocarbons in water using ionic liquid 1-methyl-3-hexylimidazolium hexafluorophosphate. Environmental Chemistry. 2005;24:467-484

[5] Tomasi J, Mennucci B, Cammi R. Quantum mechanical continuum solvation models. Chemical Reviews. 2005;105:2999-3094

[6] Paris F, Balaguer P, Terouanne B, Servant N, Lacoste C, Cravedi J, et al. Phenylphenols, biphenols, bisphenol-A and 4-tertoctylphenol exhibit and estrogen activities and antiandrogen activity in reporter cell lines. Molecular and Cellular Endocrinology. 2002;S4(193):43-48

[7] Keresztury G, Holly S, Besenyei G, Varga J, Wang A, Durig JR. Vibrational spectra of monothiocarbamates-II. IR and Raman spectra, vibrational assignment, conformational analysis and $\mathrm{ab}$ initio calculations of S-methylN,N-dimethylthiocarbamate.

Spectrochimica Acta Part A: Molecular Spectroscopy. 1993;49:2007-2026

[8] Collins LA, Boehly TR, Ding YH, Radha PB, Goncharov VN, Karasiev VV, et al. A review on ab initio studies of static, transport, and optical properties of polystyrene under extreme conditions for inertial confinement fusion applications. Physics of Plasmas. 2018;25:56306

[9] Lu T, Chen F. Multiwfn: A multifunctional wavefunction analyzer. Journal of Computational Chemistry. 2011;33:580-592

[10] Perdew JP, Kurth S, Zupan A, Blaha P. Accurate density functional with correct formal properties: A step beyond the generalized gradient approximation. Physical Review Letters. 1999;82:2544-2547

[11] Heyd J, Scuseria GE, Ernzerhof M. Hybrid functionals based on a screened coulomb potential. The Journal of Chemical Physics. 2003;118:8207-8215

[12] Hättig C, Schmitz G, Koßmann J. Auxiliary basis sets for density-fitted correlated wavefunction calculations: Weighted core-valence and ECP basis sets for post-d elements. Physical Chemistry Chemical Physics.

2012;14:6549

[13] Dennington R, Keith TA, Millam JM. GaussView Version 6. Shawnee Mission, KS: Semichem Inc; 2016

[14] Frisch MJ, Trucks GW, Schlegel HB, Scuseria GE, Robb MA, Cheeseman JR, et al. Gaussian 16 Revision A.03.

Wallingford CT: Gaussian, Inc.; 2016

[15] Parthasarathi R, Raman SS, Subramanian V, Ramasami T. Bader's electron density analysis of hydrogen bonding in secondary structural elements of protein. The Journal of Physical Chemistry A. 2007;111:7141-7148 

Section 5

\section{Quantum Mechanisms and Meta-Heuristics Concepts About Quantum Communication}





\title{
Schrödinger's Cat and His Timeless $(\mathrm{t}=0)$ Quantum World
}

\author{
Francis T.S. Yu
}

\begin{abstract}
The most famous cat in modern physics must be the Schrodinger's cat, in which he hypothesized that his cat cannot be determined alive or dead until we look into his box, by which the paradox of his half-live cat had been puzzling the quantum physicists over three quarter of a century since Schrödinger disclosed it in a Copenhagen forum in 1935. Since the disclosure, the paradox has been debated by Einstein, Bohr, Schrodinger, and many other renowned physicists, until now. We have found the cause of the paradox, and we will show in this chapter of which the hypothesis of Schrodinger's cat is not a paradox after all. It was the timeless radioactive particle he introduced into the box, since timeless and temporal spaces are mutually exclusive. We will show that the whole quantum world is timeless (i.e., $t=0$ ), since quantum mechanics can be regarded as mathematics.
\end{abstract}

Keywords: Schrödinger's cat, quantum mechanics, superposition principle, timeless subspace, temporal space

\section{Introduction}

One the most famous cats in science must be the Schrödinger's cat in quantum mechanics, in which the cat can be either alive or dead at the same time, unless we look into the Schrödinger's box. The life of Schrödinger's cat has been puzzling the quantum physicists for over eight decades as Schrödinger disclosed it in 1935. In this chapter, I will show that the paradox of the cat's life is primarily due to the underneath subspace in which the hypothetical subatomic model is submerged within a timeless empty subspace (i.e., $\mathrm{t}=0$ ). And this is the atomic model that all the particle physicists, quantum scientists, and engineers had been using for over a century, since Niels Bohr proposed it in 1913. However, the universe (our home) is a temporal space (i.e., $\mathrm{t}>0$ ), and it does not allow any timeless subspace in it. I will show that by immersing the subatomic model into a temporal subspace, instead of a timeless subspace, the situation is different. I will show that Schrödinger's cat can only either be alive or dead, but not at the same time, regardless if we look into or not look into the Schrödinger's box. Since the whole quantum space is timeless (i.e., $t=0$ ), we will show that the fundamental superposition principle fails to exist within our temporal space but only existed within a timeless virtual space. This is by no means of saying that timeless quantum space is a useless subspace. On the contrary it has produced numerous numbers of useful solutions for practical application, as long as the temporal or causality condition (i.e., t $>0$ ) is not the issue. In short, we have found the hypothesis of Schrödinger's cat is not a physical realizable 
postulation, and his quantum mechanics as well as his fundamental principle of superposition is timeless, which behaves like mathematics does.

One important aspect within our temporal universe (or time-dependent universe) $[1,2]$ is that one cannot get something from nothing: there is always a price to pay. For example, every piece of temporal subspace (or every bit of information) takes energy and time to create. And the created subspace (or substance) cannot bring back the section of time that has expensed for its creation. Every temporal subspace cannot be a subspace of an absolute empty subspace, and any absolute empty space cannot have temporal subspace in it. Any science proven within our temporal universe is physically real; otherwise, it is fictitious unless it can be repeated by experiments.

Science is a law of approximation and mathematics is an axiom of absolute certainty. Using exact math to evaluate inexact science cannot guarantee the solution exists within our temporal subspace. Science is also an axiom of logic; without logic science would be useless for practical application.

In addition, all the fundamental sciences need constant revision. For example, science has evolved from Newtonian mechanics to Einstein's theory of relativity and to Schrödinger's quantum mechanics. And the beauty of the fundamental laws must be mathematical simplicity, so that their complicated logics and significances can be understood easily. And the advantages have been very useful for extending scientific researches and their applications.

Nonetheless, practically all the particle sciences were developed from pointsingularity approximation and had been "unintentionally" embedding a pointsingularity atomic model [3] within an empty timeless subspace, as shown in Figure 1.

In which we see that, nucleus and electrons were shown by a dimensionless singularities representation. And we may not be aware that the model is not a physically real model, since the submerged background represents a timeless empty subspace. However, a timeless empty subspace cannot exist within our temporal universe! Although Bohr's atomic model have been used since the birth of Bohr's atom [3], its background has been mistakenly interpreted as an absolutely empty timeless subspace. Strictly speaking, as a whole it is not a physically correct model, and the solution

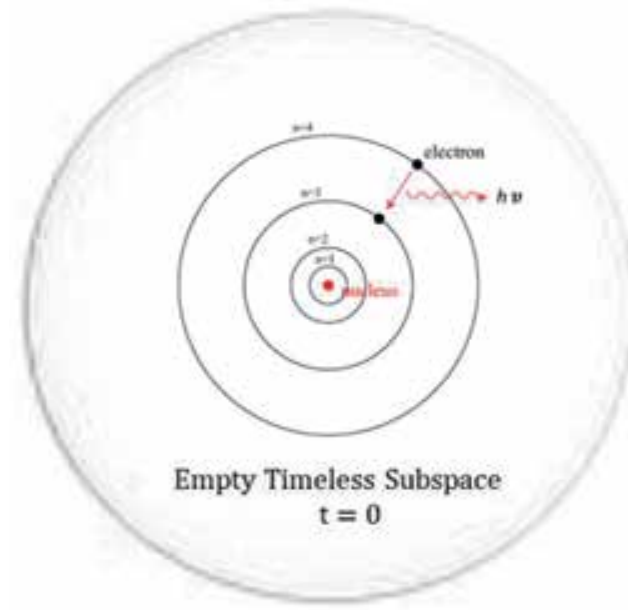

Figure 1.

An isolated Bohr's atomic model (or a timeless model); $h$ is the Planck's constant, and $v$ is the radiation frequency. 
should not be used for temporal or causality problems. The reason is that the timeless subspace model (i.e., $t=0$ ) cannot exist within our temporal space (i.e., $t>0$ ).

On the other hand, any atomic model as presented in Figure 2 is physically real, in which we see that a Bohr atom is embedded within a temporal (time-dependent) subspace (e.g., our universe).

\section{Flaws of a physical model}

Basically all the models are approximated. For example, point-singularity approximation for an atomic model offers the advantage of simplicity representation, but it deviates away from a real physical dimension, which causes the accuracy in solution. Secondly, physical model embedded within a timeless (i.e., $t=0)$ subspace is absolutely incorrect, since every physical subspace is a temporal (i.e., $t>0$ ) subspace, and it cannot be coexisted with a time-independent (or a timeless) subspace $[1,2]$. Therefore as we can see, solution obtained from a physical model embedded within a timeless empty subspace shown in Figure 2 is absolutely incorrect, and it bounds to have incomplete or fictitious solution. The fact is that one of the significant reasons other than the singularity approximation is the temporal or causality condition (i.e., $t>0$ ) which is required as we applied within our temporal universe. Therefore as depicted in Figure 1, it is not a physical realizable model, since time-dependent (or temporal) atom cannot exist within an absolute empty timeless subspace. As shown, it produces physically nonexistent fictitious solutions, which is similar as plunging a temporal machine into a nontemporal subspace.

On the other hand as referenced to Figure 2, a temporal (time-dependent) atomic model which is embedded within a time-dependent (or temporal) subspace is a physical realizable model, in which we see that the temporal or causality requirement (i.e., $t>0$ ) imposed by our temporal subspace is included. In fact our universe was created by a Big Bang explosion followed by the laws of physics, which is a temporal (i.e., $t>0$ ) universe [1, 2]. Therefore, any physical system within our temporal space has to follow the law of time (or causality condition), so that every physical science has to be proven temporal (i.e., $t>0$ ) within our universe (our home); otherwise it is a virtual fictitious science.

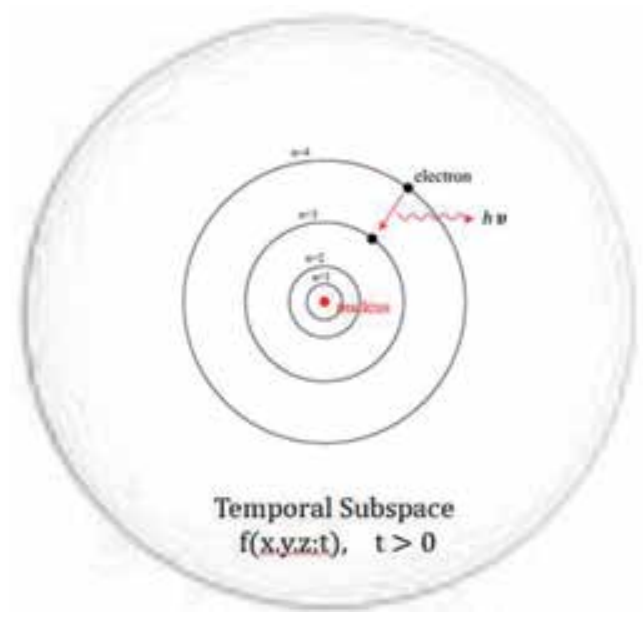

Figure 2.

An isolated atomic model embedded in temporal subspace (or a temporal atomic model). $f(x, y, z ; t) ; t>0$ represents a function of three-dimensional space and time $t$ as a forward variable. 


\section{Schrödinger's equation}

One of the most important equations in quantum mechanics must be the Schrödinger equation as given by $[4,5]$ :

$$
\frac{\partial^{2} \psi}{\partial x^{2}}+\frac{8 \pi^{2} \mathrm{~m}}{h^{2}}(E-V) \psi=0
$$

where $\boldsymbol{\psi}$ is the Schrödinger wave function, $\mathrm{m}$ is the mass, $\mathrm{E}$ is the energy, $\mathrm{V}$ is potential energy, and $h$ is Planck's constant. The description of Schrödinger equation shows that changes of a physical system over time, in which quantum effects take place, such as wave-particle duality, are significant. However, the derivation of Schrödinger equation was based on point-singularity dimensionless atomic model submerged in a timeless empty space. And we have seen that there is a contrasting paradox, by which the model used in deriving the famous Schrödinger equation is incorrect, since a time-dependent atomic structure was, by not knowing it, embedded in an absolute empty timeless subspace, for which the evaluated Schrödinger equation is also a timeless equation [5]. We note that the intention of using the timeless subspace Bohr model was inadvertent, since Bohr's atomic model has been successfully accepted, in fact for over a century, and we are still using this model. This may be the reason that causes us to overlook the basic assumption, of which a time-dependent (temporal) subspace should not be embedded in a timeless subspace, since they are mutually exclusive. Nevertheless, the essence of Schrödinger equation is to predict a particle probabilistic behavior, as a dynamic particle, by means of a wave function. In other words, the outcome is not deterministic but a distribution of possible outcomes. But the question is: Is Schrödinger equation a physically reliable equation to derive its wave equations? The answer is "no," as remained to be shown in the following:

Since the derivation of Schrödinger equation is based on point-singularity approximation which is not a perfect assumption, it is an acceptable good approximation for this hypothesis. But it is the timeless subspace of the Bohr's atomic model embedded, which produces timeless solutions (i.e., $\mathrm{t}=0$ ) that are not acceptable within the temporal (time-dependent) subspace. In other words, the solution as derived from Schrödinger equation is expected to be timeless since Schrodinger equation is a time-independent equation. Thus we see that Schrödinger's quantum mechanics is a time-independent mechanics or timeless (i.e., with respect to the absolute empty timeless subspace) mechanics, which does not exist within our temporal universe!

As quoted by Feynman [6], "He think he can safely say that nobody understands quantum mechanics. So do not take his lecture too seriously...." Yet, after we understood the flaw of Schrödinger's cat, which has haunted quantum physicists for decades, we shall take a closer look at the paradox of the Schrodinger's cat. And at that moment, we may change our mind to saying that we have learned the inconsistency of Schrödinger's timeless (i.e., $t=0$ ) quantum mechanics, as applied within our temporal universe (i.e., $\mathrm{t}>0$ ).

However, as I attempt to derive a wave dynamic where a particle is assumed situated within a temporal subspace, I am not sure that I will not be buried by complicated mathematical formulation (e.g., I have not attempted to do it yet at the time being). But I anticipate that the new result would not be paying off at least for the time being; it will have a better one than the Schrödinger equation that has already provided. But I am sure the solution will obey toward the causality condition (i.e., $t>0$ ). 
As has been done by using the Schrödinger equation to evaluate the particle wave function, one may need to reinterpret the solution to meet the causality constraint as imposed by our temporal universe. Otherwise, the evaluated solution would not be useful for practical application, in which we see that instant quantum entanglement [7] is one of the typical examples that was derived from the classic Schrödinger superposition principle. And we can see that the "instant" (i.e., t $=0$ ) entanglement between particles is "fictitious" and it would not happen within our temporal space. As we know that within our temporal universe time is distance and distance is time, any particle entanglement cannot happen instantly without a price to pay (e.g., time or distance).

As we look back to the particle model embedded in an empty subspace for deriving the classic Schrödinger equation, without such a simplistic model, viable solution may not be able to obtain even using tons of complicated mathematic manipulation. Although those assumptions alleviate (somewhat) the complexity in analysis, it also introduces incomplete results that may not exist within our universe. Thus by knowing Schrödinger's quantum mechanics, it is a time-independent (or more precisely a timeless quantum computing machine) mechanics which was the consequence of using the assumed particle model within a timeless subspace. Since in practice timeless substance cannot exist within our temporal universe, we see that the flaw of Schrödinger cat as well the whole quantum space is due to the assumption that the embedded subspace is absolutely empty, in which we see that one cannot simply insert a timeless quantum machine into a time-dependent (i.e., $\mathrm{t}>0$ ) subspace.

\section{Pauli exclusive principle and particle entanglement}

The Pauli exclusive principle [8] states that two identical particles with the same quantum state cannot occupy the same quantum state simultaneously, unless these particles exist with a different half-spin. While quantum entanglement [7] occurs when a pair of particles interacts in such a way that the quantum state of the particles cannot be independently described, even when the particles are separated by a large distance, a quantum state must be described by the pair of particles as a whole.

In view of Pauli exclusive principle, the entanglement between particles does exist, but the separation between the particles has to be limited, since the particles are situated within a time-dependent subspace (i.e., $t>0$ ) [8]. Again we see that the flaw of instant entanglement comes from the assumption that the exclusive principle was derived within the timeless subspace, in which we see again that temporal and timeless subspaces cannot coexist. In other words, time-dependent particles cannot coexist within a timeless subspace.

Before we move away from the timeless issue, we would point out that practically all of the fundamental principles in science, such as Paul's exclusive principle, Schrödinger's superposition principle, Einstein's energy equation, and others, are timeless principles, of which they were hypothesized "inadvertently" within a timeless environment.

\section{Schrödinger's cat}

One of the most intriguing cats in quantum mechanics must the Schrödinger's cat, in which it has eluded the particle physicists and quantum scientists for decades. Let us start with the Schrödinger's box as shown in Figure 3; inside the box 


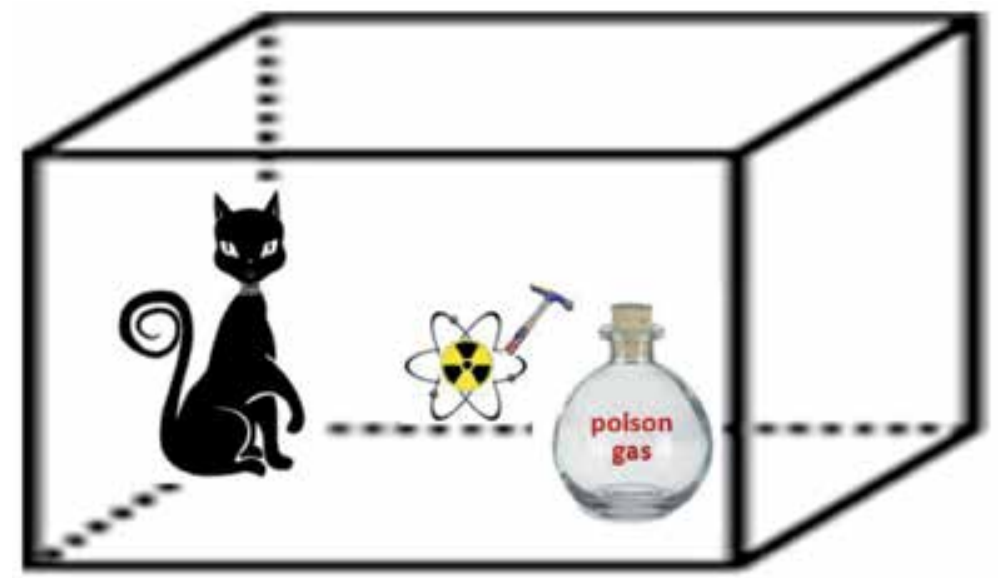

Figure 3.

Inside the box we equipped a bottle of poison gas and a device (i.e., hammer) to break the bottle, triggered by the decaying of a radioactive particle, to kill the cat.

we have equipped a bottle of poison gas and a device (i.e., a hammer) to break the bottle, triggered by the decaying of a radioactive particle, to kill the cat. The box is assumed totally opaque of which we do not know that the cat will be killed or not, as imposed by the Schrödinger's superposition principle, until we open the box.

With reference to the fundamental principle of superposition of quantum mechanics [4], the principle tells us that superposition holds for multi-quantum states in an atomic particle, of which the principle is the "core" of quantum mechanics. In other words, without the superposition principle, it will not have Schrödinger's quantum mechanics. In view of this principle, we see that the assumed two states of radioactive particle inside the box can actually simultaneously coexist, with a cloud of probability (i.e., both one thing and the other existed at the same time).

Since the hypothetical radioactive particle has two possible quantum states (i.e., decay or non-decay) that existed at the same time, which is imposed by the virtue of superposition principle in quantum mechanics, this means that the cat can be simultaneously alive and dead, before we open the box.

But as soon as we open the box, the state of superposition of the radioactive particle collapses, without proof! In an instant, we have found that after the box is opened, the cat is either alive or dead, but not both. This paradox in quantum mechanics has been intriguing particle physicists and quantum scientists over eight decades, since the birth of Schrodinger's cat in 1935, as disclosed by Erwin Schrödinger who is as famous as Albert Einstein in modern physics.

Let us momentarily accept what the fundamental principle holds, such that superposition of a dual-quantum state radioactive particle exists within the box. This tells us that the principle has created itself a timeless (i.e., $\mathrm{t}=0$ ) quantum subspace or time-independent quantum space. However, timeless subspace cannot exist within our temporal universe, in which we see that any solution (i.e., wave function) as obtained by Schrödinger equation contradicts the basic superposition principle, such that a timeless quantum subspace exists within our temporal (i.e., time-dependent) universe. This conjecture tells us that the hypothetical radioactive material cannot actually exist within the box, since both quantum states (i.e., decay or non-decay) cannot occur at the same time within a time-dependent subspace. We stress that time is distance and distance is time within a temporal subspace. 
Nevertheless, it remains a question to be asked: Where is the source that produces the timeless radioactive particle? Why is Schrodinger's superposition principle timeless (i.e., $\mathrm{t}=0$ ) for which the particle's quantum states exist simultaneously (i.e., $\mathrm{t}=0$ ) ? A trivial answer is that it has to be coming from a timeless subspace where the particle model embedded is shown in Figure 5. As we continue searching the root of paradox of the Schrödinger cat, we will provide an equivalent example to show that the paradox of the half-life cat is not a paradox.

\section{Paradox of Schrödinger's cat}

Let us replace the binary radioactive particle with a flipping coin in the Schrödinger's box shown in Figure 4.

So as one flips a coin before it is landed, it is absolutely uncertain that the coin will land either as a head or as a tail. Suppose we are able to "freeze" the flipping coin in the space at time t'; then the flipping coin is in a timeless mode subspace at time t', which is equivalent to a two-state timeless particle frizzed as time equates to t'. Then as soon as we let the flipping coin continuingly flip down at the same instance time $t=t$ ', there should be "no" lost time with respect to the time of the coin itself, but "not" with respect to the time of the box. In other words, there is a section of time $\Delta t$ that the box has gone by. So there is a time difference between the coin's time and box's time. That is precisely why we cannot tell if the cat will die or be alive, as Schrödinger himself assumed his fundamental principle is correct. As soon as we open the box, we have to accept the physical consequence that the cat is either dead or alive, but not both. Then I guess Schrödinger creates a logic to save his fundamental principle that superposition of the radioactive particle quantum states suddenly "collapses" as we open the box, without any physical proof. Otherwise the core of quantum mechanics fails to live up with the physical reality. Nevertheless as we see it, the failure of the fundamental principle is due to the fact that a timeless flipping coin "cannot be coexisted" within a timedependent (i.e., $t>0$ ) box.

We further note that it is possible to alleviate the timelessness of superposition, if we appropriately add the temporal constraint (i.e., $t>0$ ) in deriving the Schrödinger equation. We can change the timeless Schrödinger's equation to a

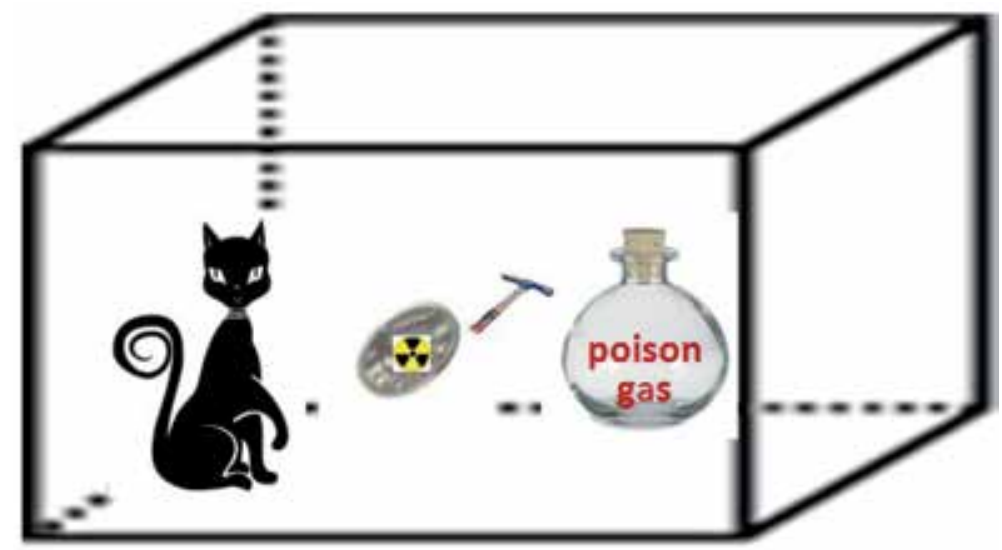

Figure 4.

A flipping coin analogy is substituted in the box for Schrödinger's cat paradox. 


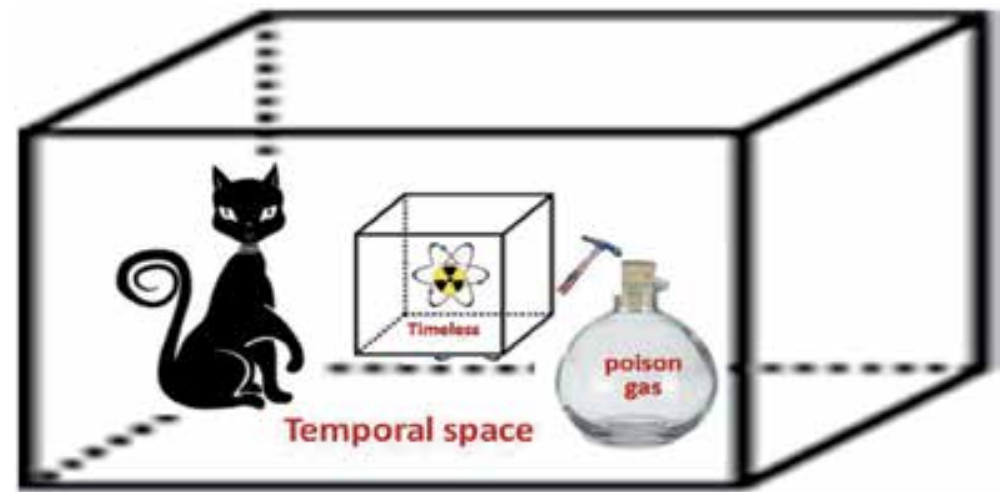

Figure 5.

Schrödinger's box with a timeless radioactive particle. Notice that timeless radioactive particle cannot exist in a temporal (i.e., time-dependent) subspace.

time-dependent (i.e., $t>0$ ) equation, of which we will see that Schrödinger's wave functions of the dual-state radioactive particle can be shown as $\boldsymbol{\psi}_{1}(\mathrm{t})$ and $\boldsymbol{\psi}_{2}$ $(\mathrm{t}+\Delta \mathrm{t})$, respectively, where $\Delta \mathrm{t}$ represents a time delay between them. Since time is distance and distance within a temporal subspace, we see that the quantum states will not occur at the same time (i.e., $t=0$ ). Furthermore, the degree of their mutual superposition states can be shown as a time ensemble of $\left\langle\psi_{1}(\mathrm{t}) \psi_{2}{ }^{*}(\mathrm{t}+\Delta \mathrm{t})\right\rangle$, respectively, where ${ }^{*}$ denotes the complex conjugate, in which we see that a perfect degree of mutual superimposition occurs if and only if $\Delta \mathrm{t}=0$, which corresponds to the timeless (i.e., $t=0$ ) quantum state of the radioactive particle.

Now let us go back to the half-live cat in Schrödinger's box, where the radioactive particle is assumed within a timeless sub-box as shown in Figure 5, in which we see that a timeless (i.e., $\mathrm{t}=0$ ) radioactive particle is situated inside the time-dependent (i.e., $\mathrm{t}>0$ ) box, which is "not" a physical realizable postulation for Schrödinger's cat. The fact is that a timeless $(t=0)$ subspace cannot exist within a time-dependent $(t>0)$ space (i.e., the box). Thus we have shown that again the paradox of Schrödinger's is not a paradox, since the postulated superposition is timeless, and it is not a physical realizable principle within our temporal universe!

However, by replacing the timeless particle with a time-dependent (i.e., $t>0$ ) particle shown in Figure 5, then we see there is a match in time as a variable with respect to the box. Then Schrödinger's cat can only either be dead or not be dead but not at the same time, in which we see that there is nothing to do whether we open the box or not to cause the fundamental principle to collapse. In other words, a dead cat or a live cat has already been determined before we open up the box. And the occurrence of the particle's quantum states is not simultaneously by means of the fundamental principle of Schrödinger, in which we have shown that superposition principle does not exist within our temporal space and it only exists within a timeless virtual subspace similar to what mathematics does.

At last, we have found the flaw of Schrödinger's cat, where Schrödinger was not supposed to introduce a timeless radioactive particle into the box. This vital mistake that he committed is apparently due to an atomic model in which subspace is assumed to be absolutely empty as shown in Figure 1, in which we see that a timeless (i.e., $t=0$ ) particle is wrongly inserted into a temporal (i.e., $t>0$ ) box. I believe we have finally found the root of the paradox of Schrödinger's cat, for which we shall leave the cat behind with a story to tell; once upon a time, there was a half-life cat! 


\section{Essence of a subatomic model}

With high degree of certainty, most of the fundamental laws of science embraced the singularity approximation which includes the atomic models embedded within a timeless subspace. As we look at any conventional atomic model, we might inadvertently assume that the background subspace is an absolutely empty space. And this is the consequence of Schrödinger's timeless quantum mechanics, since any physical atom (i.e., $\mathrm{t}>0$ ) cannot be situated within a timeless (i.e., $t=0)$ subspace. Although singularity model works very well for scores of quantum mechanical application until the postulation of Schrödinger's cat emerged. Since the paradox of the half-life cat is the core of the fundamental principle, it has been argued for over eight decades by Einstein, Bohr, Schrödinger, and many others since Schrödinger disclosed the postulation at a Copenhagen forum in 1935. This intrigues us to look at Schrödinger's equation which was developed on an empty (i.e., $\mathrm{t}=0$ ) subspace platform, in which we see that superposition position collapses as soon as we open Schrödinger's box. This must be the apparent justification for Schrödinger to preserve the fate of his fundamental principle. Otherwise his timeless fundamental principle cannot survive within our temporal universe (i.e., $t>0$ ). In short, we see that the hypotheses of Schrödinger's cat are a fictitious postulation, and we have proof that it does not have a viable physical solution, since any timeless radioactive particle cannot coexist in a temporal box, and we have seen that Schrödinger have had inadvertently introduced in the box (Figure 6).

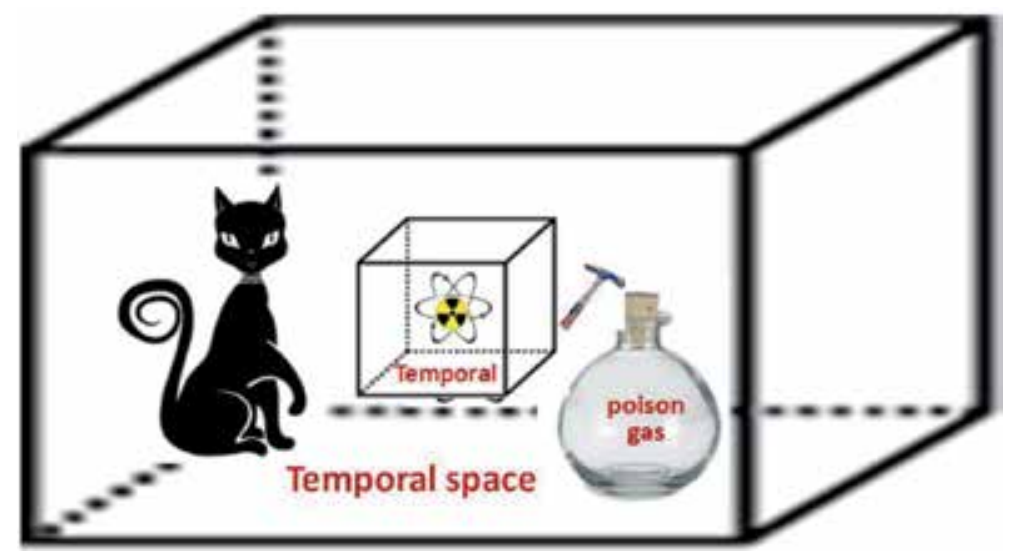

Figure 6.

A time-dependent cat is in a temporal (time-depending) box, in which we see a temporal radioactive particle is introduced within Schrodinger's temporal box.

\section{Timeless quantum world}

Fundamental principle of quantum mechanics tells us that superposition of a multi-quantum-state particle holds if and only if within a quantum environment, by which it creates itself a timeless quantum subspace, but quantum subspaces cannot exist within our temporal universe. Then there is a question being asked: Can those quantum subspaces be utilized in our temporal universe? The answer is "no" and "yes."

The "no" part answer is that if time component in application is an issue, such as applied to "instant" quantum entanglement [9] and "simultaneous" quantum 
states computing [10], then the superposition principle as derived from the timeindependent Schrödinger equation would have a problem, as applied within our temporal universe, since the superposition is timeless. For example, those instant and simultaneous response promises by the fundamental principle do not exist within our temporal space. And the postulated Schrödinger's cat is not a physical realizable solution, in which we have shown that the burden of the cat's half-life can be liberated by using a temporal (i.e., $\mathrm{t}>0$ ) radioactive particle instead, in which we see that the paradox of Schrödinger's cat may never be discovered that it is not a paradox, if we did not discover that Schrödinger's quantum mechanics is timeless.

Since the Schrödinger equation is a timeless quantum computer, which is designed to solve a variety of particle's quantum dynamics, the solution as obtained from Schrodinger's equation is also timeless, which produces a non-realizable solution such as timeless (i.e., $\mathrm{t}=0$ ) superposition.

We see that if one forces a timeless (i.e., $t=0$ ) solution into a temporal (i.e., $\mathrm{t}>0$ ) subspace, one would anticipate paradox solution that does not exist within our temporal universe, such as Schrödinger's half-live cat. This is equivalent to chasing a ghost of a timeless half-life cat in a temporal subspace, in which we have found that a timeless radioactive particle was inserted within Schrödinger's box!

As to answer the "yes" part, if temporal aspect as applying a quantum mechanical solution is not an issue within our temporal space, then we have already seen scores of solutions as obtained from the Schrödinger equation which have been brought to use in practice, since the birth of quantum mechanics in 1933. This is similar to using mathematics (i.e., a timeless machine) to obtain solution for timedependent application and sometime produces solution not physically realizable, in which we see that the Schrödinger equation is a mathematics, which requires a time boundary condition (i.e., $t>0$ ) to justify that its solution is physically realizable.

\section{Math and temporal $(t>0)$ space duality}

Every physical science existed within our temporal subspace must be temporal (i.e., $\mathrm{t}>0$ ); otherwise, it is a virtual (or fictitious) science as mathematics does. The burden of a scientific postulation is to prove it exists within our universe and then find the solution. We shall now show that there exists a duality between science and mathematics in which any scientific hypothesis has to be shown that it is within the boundary condition of our temporal universe, before accepting it as a real postulation. Otherwise, the hypothesis is not a guarantee to be physically real. One of the essential boundary conditions is the causality condition (i.e., $t>0$ ), which is to show that the solution is temporal and causal (i.e., $t>0$ ). For instance, take Einstein's energy equation [11] as an example as given by.

$$
\boldsymbol{\varepsilon}=\mathrm{mc}^{2}
$$

where $\mathrm{m}$ is the rest mass and $\mathrm{c}$ is the speed of light. In view of this equation, we first see that it is not a temporal or time-domain function. Strictly speaking, this equation cannot be directly implemented within our temporal subspace, since our universe is a temporal variable spatial function which can be described by $[1,2]$.

$$
f(x, y, z ; t), t>0
$$

where $(\mathrm{x}, \mathrm{y}, \mathrm{z})$ is a spatial variable and $(\mathrm{t}>0)$ is a forward time variable, in which we see that every subspace within our universe is time-dependent variable 
space. Since energy equation of Eq. () is not time variable equation, it is apparent that the equation cannot be directly implemented within our temporal universe. To make the energy equation be acceptable or match to our temporal (i.e., $t>0$ ) subspace condition, we can transform the equation to become time-domain or temporal equation as given by [].

$$
\frac{\partial \varepsilon(t)}{\partial t}=c^{2} \frac{\partial m(t)}{\partial t}, \mathrm{t}>0
$$

where $\partial \varepsilon(t) / \partial t$ is the rate of increasing energy conversion, $\partial m(t) / \partial t$ is the corresponding rate of mass reduction, $c$ is the speed of light, and $t>0$ represents a forward time variable. Notice that we have transformed the equation into a partial differential form which exists only at time $t>0$. This indicates that the solution as obtained by this equation is compiled by means of the causality (i.e., $t>0$ ) constraint, of which the solution can be used within our temporal universe (i.e., $t>0$ ).

On the other hand, if Eq. (4) is imposed by a timeless (i.e., $t=0$ ) constraint as shown by

$$
\frac{\partial \varepsilon(t)}{\partial t}=c^{2} \frac{\partial m(t)}{\partial t}, \mathrm{t}=0
$$

then we see that the solution as obtained by Eq. (5) will be timeless (i.e., existed at $t=0$ ). And it cannot be implemented within our temporal (i.e., $t>0$ ) universe.

Needless to say, if we put a constraint on Eq. (3) as can be shown by $f(x, y, z ; t)$, $t=0$. Then we see that a temporal equation has been transformed into a timeless equation which exists only at $\mathrm{t}=0$, in which we see that Eq. (5) cannot be used within our temporal universe (i.e., $t>0$ ).

As we know that a timeless space is actually a mathematical virtual space, only mathematician and possibly quantum physicist can produce it, since quantum mechanics is mathematics. Nevertheless, a timeless space has no time and no substance in it. When we look back at all the fundamental laws in science, they are mostly presented by point-singularity approximation, and many of them are timeless or time-independent equations, such as Schrödinger's equation. And we have shown in proceeding that Schrodinger's quantum machine is timeless since its mechanics was built on an empty subspace. Nevertheless we are going to show some possible outcome when a timeless superposition principle is implemented within a timeless platform. Before showing, let us introduce a few subspaces that may be used for the illustration, as depicted in Figure 7.

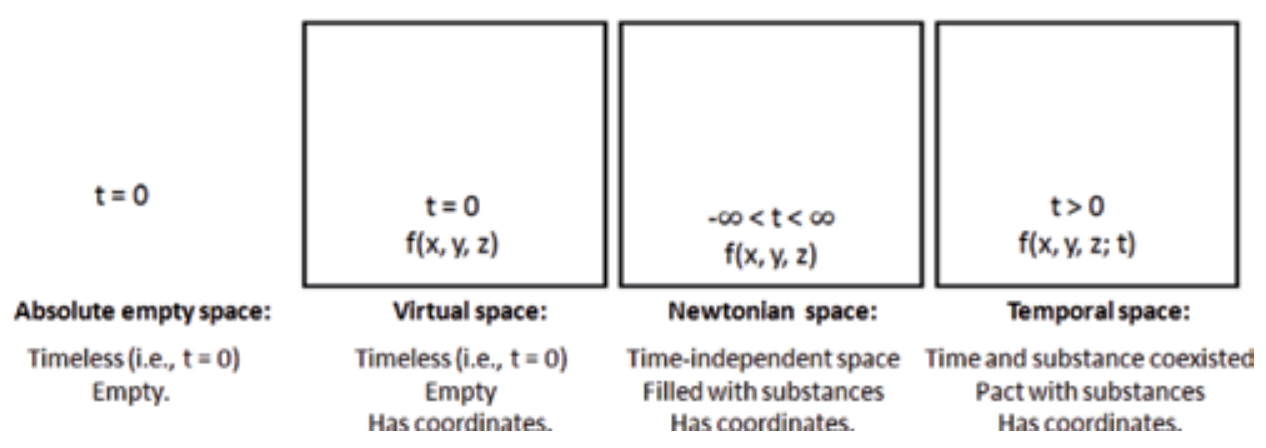

Figure 7.

This figure shows an absolute empty space (a), a virtual space (b), a Newtonian space (c), and Yu's temporal space (d). 
In Ref. to this figure, we see an absolute empty space which has no time, no substance, and no coordinate. A mathematical virtual space is an empty and timeless space with spatial coordinates. A Newtonian space is filled with substance but treated time as an independent variable. And finally a temporal space is filled with substance and existed only at $t>0$, of which substance and time coexisted $[1,2]$. We further see that none of the spaces such as absolutely empty, virtual, and Newtonian spaces can be a subspace of the temporal space or vice versa, since temporal (i.e., $t>0$ ) space is a time-invariant system (i.e., the system analysis standpoint) and the others are not.

Now, let us take an example as illustrated in Figure 8 in which we assume three delta functions $\delta\left(\mathrm{t}-\mathrm{t}_{1}\right), \delta\left(\mathrm{t}-\mathrm{t}_{2}\right)$, and $\delta\left(\mathrm{t}-\mathrm{t}_{3}\right)$ representing a set of particles that are plunging into a timeless subspace system diagram as depicted in Figure $\mathbf{8 b}$. We see that output delta functions are superimposed on top of each other at $t=0$, shown in Figure 8c, of which we note that all the input pulses (i.e., particles) lost their temporal identities within a timeless space. And this is precisely the superposition principle tells us that the entire quantum states exist simultaneously and instantly (i.e., at $\mathrm{t}=0$ ). However, superposition principle does not exist within a temporal (i.e., $\mathrm{t}>0$ ) space. Since time is distance and distance is time, the entire quantum states exist simultaneously everywhere only within a timeless space as can be seen in Figure 8e. Therefore, it is a serious mistake to assume superposition principle works within our temporal universe, such as the paradox of Schrödinger's cat and possibly others. It is interesting to find out from system analysis standpoint [3] how a timeless (i.e., $\mathrm{t}=0$ ) subspace respond to a time-domain input excitation.

On the other hand, if we plunge the delta pulses within a temporal subspace, as shown in Figure 9, we see the output responses are faithfully temporally reproduced, which shows the time-invariant property of our temporal subspace, in which these particles (e.g., quantum states) are temporally separated, instead of superposing together at $\mathrm{t}=0$. And this is precisely the moment when we open Schrodinger's box, we found the cat can only be either dead or alive but not both at the same time. Instead of assuming the fundamental principle collapses to justify the superposition principle.

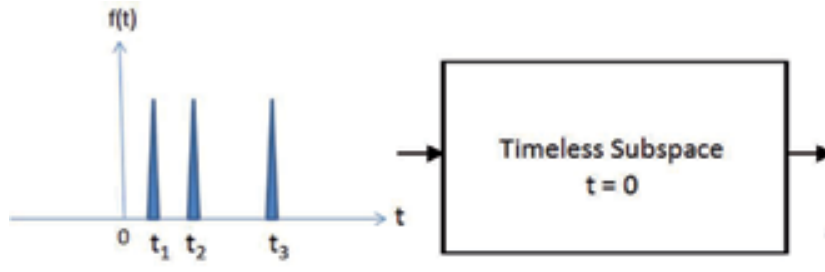

(a) Input temporal excitation

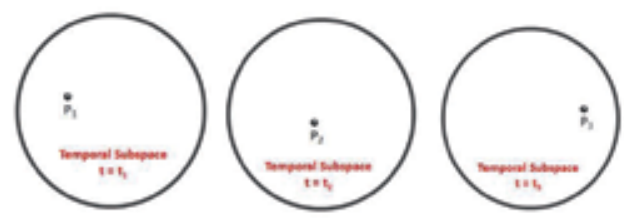

(d)Input temporal domain representation (b) Block box representation

(c) Output timeless response

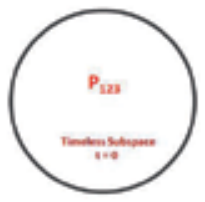

(e) Output timeless domain representation

Figure 8.

(a) Shows a set of three pulses (e.g., particles) within a temporal subspace as shown in topographical view in $(d)$. As these particles plunge into a timeless subspace of $(b)$, the output responses are superposing at $t=0$ shown in (c), and the superimposed particles can be found all over the timeless domain as can be seen in (e). It is interesting to note that within a timeless space, all things are in one and one is everywhere within the space. 
In summing up our illustration, our universe is a causal (i.e., $t>0$ ) timeinvariant system which can be symbolically described by $f(x, y, z ; t), t>0$, in which time and space coexisted. Since time is a constant forwarded variable, the speed of time is determined by the velocity of light as given by $\mathrm{t} \approx 1 / \mathrm{c}$, where $\mathrm{c} \approx 186,282 \mathrm{miles} / \mathrm{sec}$, by which our temporal universe was indeed created by means of Einstein energy equation that was derived with his relativity theory, in which we see that time is distance and distance is time within our temporal universe. In contrast within a timeless (i.e., $t=0$ ) space, it has no time and no distance, since $d=c t$ and $t=0$, for which everything collapses instantly at $t=0$ (or $\mathrm{d}=0$ ) within a timeless space, as superposition principle does. Although scores of quantum mechanical solutions have been put into use, it is the fundamental principle of superposition that confronted with the temporal boundary condition t $>0$ that produces Schrödinger's cat.

Regardless the mutual exclusive issues between timeless and temporal subspaces, some quantum scientists still believe they can implant superposition principle within our universe. This is the reason that we would show what would happen when a multi-quantum states particle is implemented within a temporal space. For simplicity, we will simulate a two-quantum states particle plunging into an empty subspace as shown in Figure 10a and $\mathbf{b}$. We further let two quantum states associated with two eigenfunctions exp. $\left[i\left(\omega_{1} t\right)\right]$ and exp. $\left[i\left(\omega_{2} t\right)\right]$, where $\omega$ represents the angular frequency of the quantum state. And the output response from an empty space is given in Figure 10c that corresponds to a "timeless" superposing dualquantum state (a real quantity), where we assume energy is conserved. When this timeless simulated response is plunged into a temporal (i.e., $\mathrm{t}>0$ ) space as depicted in Figure 10d, its output response is shown in Figure 10e, in which we note that the output response occurs at $\mathrm{t}>0$ and it was not started instantly at $\mathrm{t}=0$, since time is distance and distance is time within a temporal space. In view of this simulation, we learn that particle's quantum states lost their personalities as soon it plunges into a timeless space. Since the timeless subspace is assumed to be within a temporal (i.e., $\mathrm{t}>0$ ) space, it is the temporal space that dictates the end response, as shown in Figure 10e. This shows us that all the "instance and simultaneous" quantum states as indicated by the superposition principle are not happening. Equivalently
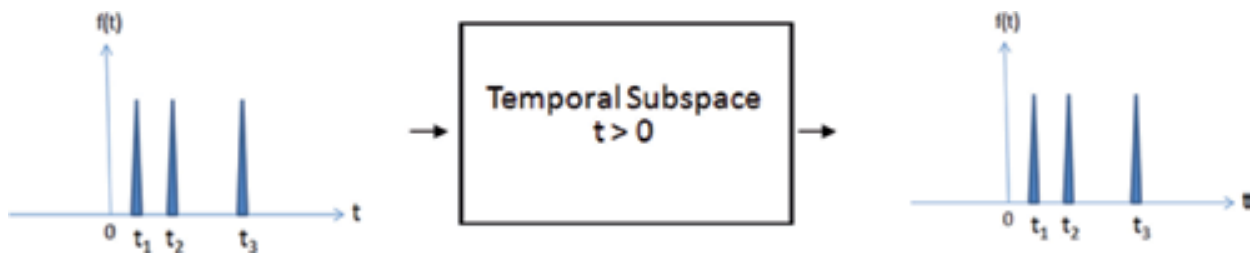

Figure 9.

The time-invariant response property from a temporal subspace.

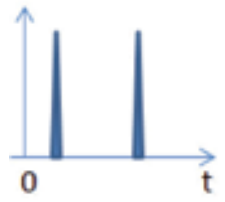

(a)

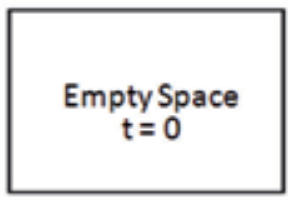

(b)

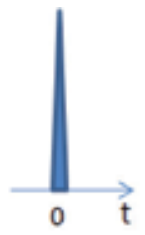

(c)

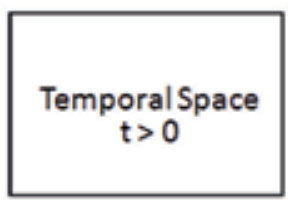

(d)

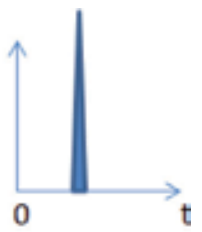

(e)

Figure 10.

System simulation for an empty subspace within a temporal space. (a) Input excitation, (b) empty timeless system, (c) output response from an empty space, (d) temporal system, and (e) final output from a temporal space. 
speaking, this is precisely why the dual-quantum states of the radioactive particle within Schrodinger's box are dysfunctional or impaired, within a temporal space.

\section{Quantum mechanical assessments}

The Schrödinger equation was developed on an absolute timeless subspace platform, for which all the solutions are timeless or time-independent. Since the fundamental principle of superposition was derived from the timeless Schrodinger equation, the corresponding quantum states' wave functions are also timeless with respect to the subspace that the particle is embedded in. Although wave function is time-dependent equation, it is with respect to the corresponding quantum state itself. This can be easily understood by an atomic model where the particle quantum states are represented by $h v_{\mathrm{n}}$, where $\mathrm{n}=1,2, \ldots \mathrm{N}$, number of quantum state, in which we see that each $\mathrm{n}$-th wave function is time dependent with respect to $h v_{\mathrm{n}}$ quantum state. And it is not with respect to the subspace that the atomic model is embedded in, which is an empty subspace. Since time-dependent wave functions dictate the legitimacy of the superposition principle, the time dependency with respect to the particle's subspace is timeless, which is precisely the reason the fundamental principle of superposition is timeless and the whole Schrodinger's quantum world is timeless (i.e., $\mathrm{t}=0$ ).

Since the whole quantum space is timeless, it cannot coexist within our temporal universe. In view of the logic of collapsing superposition principle as soon as we open up the Schrödinger's box, it must satisfy the physical reality that the cat cannot be alive and dead at the same time. Otherwise, the fundamental principle of superposition has proven itself to not exist within our temporal (i.e., $t>0$ ) universe. It is apparent that Schrödinger's fundamental principle only exists within a timeless subspace. Personally I believe this must be the reason for him to justify the fate of his fundamental principle; otherwise, the principle is not able to survive. It must be Schrödinger himself that made the argument; otherwise, the paradox of his halflife cat has no physical foundation to debate by the world's top scientists over three quarter of a century, since 1935.

Since quantum mechanics is a virtual quantum machine as mathematics is, we have found that Schrödinger's machine is a timeless (or a virtual quantum) computer and it does not exist within our temporal universe. As we have seen, the Schrödinger equation was derived within an empty subspace; it is not a physical realizable model to use, since empty subspace and non-empty subspace are mutually exclusive. And we have seen that, as one plunges the timeless superposition principle within a temporal (i.e., $\mathrm{t}>0$ ) subspace and then anticipates the timeless superposition to behave "timelessly" within a temporal subspace is physically impossible. We have shown that only mathematician and quantum mechanists can do it, since quantum mechanics is mathematics.

But this is by no means to say that timeless quantum mechanics is useless, since it has proven to us with scores of practical application that long solutions are not directly confronted with time-dependent or causality (i.e., $t>0$ ) issue within our temporal universe. As quoted by the late Richard Feynman [12] that "nobody understands part of quantum mechanics," we have found the part of quantum mechanics nobody understands which must be from the "timeless superposition principle" that causes the confusion. And the root of timelessness quantum world is from the empty subspace that the atomic model was inadvertently anchored on. We are sure this discovery would change our perception as applying the fundamental principle to quantum computing and to quantum entanglement in communication, for which the "instance and simultaneous" (i.e., $t=0$ and concurrent) 
phenomena as promised by the fundamental principle do not exist within our temporal universe. The important fallout from this discovery of the non-paradox of Schrödinger's cat encourages us to look for a new time-dependent quantum machine, similar to the one that Schrödinger has already paved the roadmap for us.

\section{Remarks}

In conclusion, I have shown that the atomic model that Schrödinger used must be anchored within an absolute empty subspace. And it must be the underneath timeless subspace that caused the paradox of his half-life cat. The reason for overlooking the underneath timeless subspace must be due to the well-accepted Bohr's model that has been used for over a century, since the birth of Niels Bohr's atom in 1913 [3]. It has been very successfully used with excellent results for over a century. And it has never in our wildness dream that the underneath empty subspace causes the problem. In view of Schrödinger's time-dependent wave solutions, we have found the time dependency is with respect to the atomic particle itself but not with respect to the subspace the atomic model embedded in. In searching the root of the paradox of Schrödinger's cat, we found that a timeless radioactive particle should not have had introduced within a time-dependent (or temporal) Schrödinger's box. To alleviate the timeless radioactive particle issue, we have replaced a timedependent (i.e., $t>0$ ) radioactive particle for which we have shown that the paradox Schrödinger's cat is not a paradox after all. We have also used science and math duality analogy to illustrate the outcome of a temporal excitation into a timeless system analog, as well as onto a temporal subspace, in which we have shown temporal space is a time-invariant space, while superposition principle is timeless and it is neither a time-invariant nor time-variant principle. It is however a no-time or timeless principle, which cannot be implemented within a time-invariant space. In short, we found the hypothesis of Schrödinger's cat is not a physical realizable postulation and his whole quantum world is timeless and behaves like mathematics does. Nonetheless, many of Schrodinger's timeless solutions are very useful until the implementation of fundamental principle that confronts with causality (i.e., $t>0$ ) issue of our universe.

\section{Author details}

Francis T.S. Yu

Emeritus Evan Pugh (University) Professor, Penn State University, University Park, Pennsylvania, USA

*Address all correspondence to: fty1@psu.edu

IntechOpen

(C) 2019 The Author(s). Licensee IntechOpen. This chapter is distributed under the terms of the Creative Commons Attribution License (http://creativecommons.org/licenses/ by/3.0), which permits unrestricted use, distribution, and reproduction in any medium, provided the original work is properly cited. (cc) BY 


\section{References}

[1] Yu FTS. Time: The enigma of space. Asian Journal of Physics. 2017;26(3):149-158

[2] Yu FTS. Entropy and Information Optics: Connecting Information and Time. 2nd ed. Boca Raton, FL: CRC Press; 2017. pp. 171-176

[3] Bohr N. On the constitution of atoms and molecules. Philosophical Magazine. 1913;26(1):1-23

[4] Schrödinger E. Probability relations between separated systems. Mathematical Proceedings of the Cambridge Philosophical Society. 1936;32(3):446-452

[5] Susskind L, Friedman A. Quantum Mechanics. New York: Basic Books; 2014. p. 119

[6] Feynman RP, Leigton RB, Sands M. Feynman Lectures on Physics. In: Quantum Mechanics. Vol. 3. Cambridge, Massachusetts: Addison-Wesley Publishing Company; 1966

[7] Bennett CH. Quantum information and computation. Physics Today. 1995;48(10):24-30

[8] Pauli W. Über den Zusammenhang des Abschlusses der Elektronengruppen im Atom mit der Komplexstruktur der Spektren. Zeitschrift für Physik. 1925;31:765

[9] Życzkowski K, Horodecki P, Horodecki M, Horodecki R. Dynamics of quantum entanglement. Physical Review A. 2001;65:012101

[10] Ladd TD, Jelezko F, Laflamme R, Nakamura C, Monroe C, O’Brien JL. Quantum Computers. Nature. 2010;464:45-53

[11] Einstein A. Relativity, the Special and General Theory. New York: Crown Publishers; 1961
[12] Feynman RP, Leighton RB, Sands M. The Feynman Lectures on Physics. Cambridge, Massachusetts: Addison Wesley; 1970 


\title{
Chapter 9
}

\section{What Is "Wrong" with Current Theoretical Physicists?}

\author{
Francis T.S. Yu
}

\begin{abstract}
Theoretical physics uses amazing mathematical paradigm and added with fantastic computer animation provides very convincing results. But mathematical modeling and computer animation are virtual and fictitious, for which many of their analytical solutions are not physically real. What is wrong with current theoretical physicists is that they have used mostly a timeless $(t=0)$ mathematical subspace for their analyses that does not exist within our temporal $(t>0)$ universe. The reason is it is not how rigorous and fancies the mathematics (or computer simulation) are; it is the essence of a physical realizable paradigm. For instance, timeless $(t=0)$ model has been used since the beginning of science; although it has produced uncountable excellent results, it has also produced many solutions that are timeless $(t=0)$ or nonexistent solutions within our temporal $(t>0)$ universe. In this article, I will show a few evidences that the theoretical analyses have done to physics, which includes some of the world-renowned theoretical scientists, past and present. Yet, theoretical physicists were and still are the creators for all the fundamental laws and principles of physics; it is their "responsibility" to take us back to the physical realizable world of science; otherwise we will be still trapped within a virtual timeless $(t=0)$ land of mathematics. In short, I anticipate that Temporal $(t>0)$ Physics will be a mainstream realizable physics in the years to come.
\end{abstract}

Keywords: theoretical physics, temporal space, timeless space, virtual space, Newtonian space, physical realizable, quantum mechanics, cosmology, relativity

\section{Introduction}

In mathematics, every postulation needs a proof it exists-a solution before searching for the solution. Yet in science, it seems to me it does not have a criterion as mathematics does, to prove first a hypothesis exists within our temporal $(t>0)$ universe. Without such a criterion, fictitious science emerges, as already have been happening in every day's event. And this is one the objectives for writing this article, in which I will show what is wrong with theoretical physicists, although they were the creators of science. Yet, all laws of physics were made to be broken and to be revised, which includes the paradoxes and principles; this is all about science. Since all the physical laws, principles, and paradigms have been working well in the past, it is by no means that they are still assumed applicable, as space is getting larger and particles are becoming smaller. As more and more sophisticated physics are discovered, the need for updating the laws and paradigms is inevitable. Otherwise virtual and fictitious solution emerges, since theoretical physics is an applied mathematics. 
Theoretical physicists were the creators of modern science and still are; it is their responsibility in part to correct what they have done to the physics recently. However, they seem to know precisely that some of their analytical solutions were irrational and spooky as have been noted by Einstein, a century ago. Yet they have not tried hard enough to find out the cause, continuingly producing those dysfunctional solutions and pretending there are real and existing within our universe, for which we have seen scores of fictitious sciences emerged as orchestrated by their ambition; that has become the mainstream of scientific research topics, for example, quantum supremacy in computing, granular time variable, curving time-space, repeated cyclic universal, and so on, in which none of them, as will be shown in subsequent discussion, actually existed with our temporal $(t>0)$ universe.

\section{Evidences}

The fact is that it is not how rigorous the mathematics (or computer simulation) is; it is the essence of a physical realizable paradigm. For instance, virtual empty space model (i.e., a mathematical space) has been used over centuries by a score of world-renowned scientists and theoretical physicists at the dawn of science. And the empty space paradigm is still used today by the theoretical physicists, "inadvertently" not knowing it is a virtual subspace not existing within our universe. As we have seen, empty space paradigm has provided not just for theoretical physicists and all of us with an impressive account of viable solutions. Since the virtual emptiness subspace comes very naturally on a piece of scratched paper but not knowing the physics behind the shadow of mathematical model is a physically unreal subspace suppose "not" to be used, but unintentionally we have been using it since the beginning of science, because science is also mathematics.

Until recently, I have discovered a nonphysical solution had been derived from an atomic model that had been drawn on a piece of scratch paper over a century ago [1] of which a paradox of Schrödinger's cat $[2,3]$ emerged. Although empty space paradigm has given scores of applicable results, it has also produced many solutions that are irrational and strange that Einstein called them spooky. And these must be the physical evidential results that have been suggested; something is very wrong in view of all the illogical consequences, as in contrast with the physical reality. But to remedy the spookiness comments from one of the world's most prestigious scientists at that time, theoretical physicists come up a very convincing answer; particles behave strangely in micro space as in contrast within macro space environment, although micro particles have been successfully applied in macro space. And this must be the powerful and convincing justifiable reason, discouraging for further investigation, although intensive debates have been started by Einstein, Bohr, Schrödinger, and many others in a scientific forum at Copenhagen in 1935 [3], and the paradox of Schrödinger's cat is still lingering today. Late Richard Feynman has said that: "After you have learned quantum mechanics, you really do not understand quantum mechanics" [4]. But most of the theoretical physicists still believe their solutions are physically real, since a score of their solutions have been successfully applied in practice.

Although I am not a physicist, I have found a score of solutions as obtained from an empty space paradigm are timeless $(t=0)$ recently $[5,6]$; strictly speaking one should not implement timeless $(t=0)$ solutions directly within our temporal $(t>0)$ space, such as superposition principle of quantum mechanics $[2,3]$. Nevertheless, some timeless $(t=0)$ solutions can be used, but not directly plunged, into our temporal $(t>0)$ universe, such as Einstein energy equation [7] as I will show later. 
Since theoretical physicists need mathematics, but mathematics is not physics, unless her analytical solution is complied within the boundary condition of our universe, causality ( $t>0$ ) and dimensionality, then her analytical solution is a physical realizable solution that can be applied directly within our universe.

\section{Burden of theoretical analysis}

As we know, physics is physically real and mathematics is abstractly virtual. However, it is not how fancy mathematics is compared to physics that guarantees her analytical solution is physically real. It is her physical realizable paradigm that determines her solution is physical realizable. In other words, if one uses an empty subspace model to evaluate a physical problem, then very likely her analytical solution will be timeless $(t=0)$. For example, using Schrödinger wave equation to analyze the quantum dynamic behavior of a particle, then the particle dynamic solution will be timeless $(t=0)$ with respect to the empty space of the model, since empty space is a timeless space.

For instances; in view of all the sophisticated mathematics such as; Hilbert space, Banach space, Riemann surface, topological spaces, group theory and others have been used by theoretical physicists, but without any physical evidence to support the solutions are physical real. Besides all those fancy mathematics were not originated by theoretical physicists but by a group of abstract mathematicians, in which we see that theoretical physics is actually an "applied" mathematics or simply mathematics.

Since theoretical physics is mathematics, the burden on their shoulders is to provide us with physically real solutions for practical implementation. Yet they have been persistently giving us the virtual fictitious solutions, even though they knew some of their results are irrational and spooky as noted by Einstein a century ago!

It seems to me it is kind of out of control; we have seen fictitious sciences added with very convincing computer animations becoming the mainstream of current topics of science. Therefore it is an urgent responsibility for the theoretical physicists to bring back the theoretical physics to reality, since the origin of physics was started by theoretical physicists.

\subsection{Subspaces}

Before illustrating the consequences as will be used for analytical solution, such as from virtual mathematical empty space paradigm, I would introduce several subspaces that have been used by the theoretical physicists in the past and present, as depicted in Figure 1.

In this figure we see an absolute-empty space, a mathematical virtual space, a Newtonian's space, and a temporal ( $t>0)$ space. An absolute-empty space or just empty space has no substance and has no time. A mathematical virtual space is an empty space which has no substance in it, but a mathematician can assume coordinates in it, since mathematics is a virtual space. Although this virtual mathematical space has been extensively used by scientists, theoretical physicists, and others since the birth of science, it is an abstract space that does not exist within our temporal (i.e., $t>0$ ) space. The next subspace is known as Newtonian space [8]; it has substance and coordinates in it but treated time as an "independent" variable. But Newtonian space does not actually exist within our temporal $(t>0)$ space, since time and substance are mutually coexisting within our temporal $(\mathrm{t}>0)$ universe. The last subspace is known as temporal $(t>0)$ space $[5,6]$, where time and substance are interdependent or coexisting, in which we note that time is a forward 


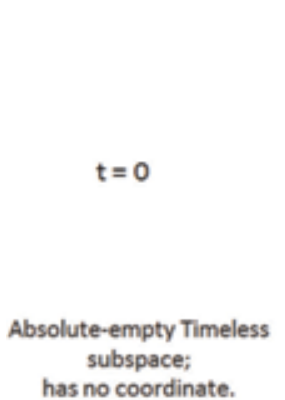

(a)
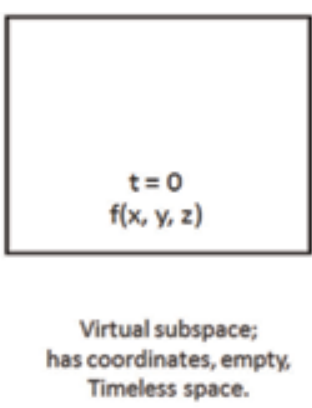

(b)
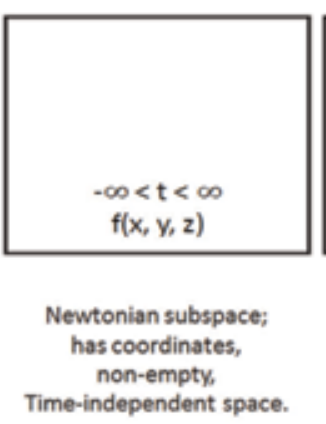

(c)

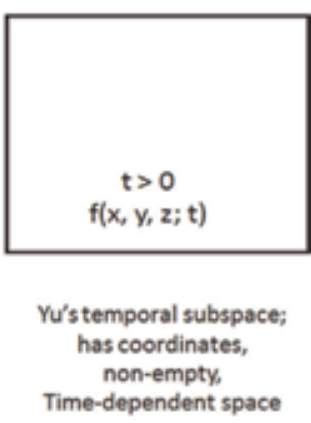

(d)

Figure 1.

(a) An absolute-empty space, (b) a virtual mathematical space, (c) a Newtonian space, and (d) a temporal $(t>0)$ space.

"independent" variable moving at a constant speed dictated by the speed of light. I stress that this temporal $(t>0)$ subspace is the "only" viable physical realizable space at this time, of which temporal $(t>0)$ space is created by the current laws of physics as derived from Einstein's theory of relativity [7].

A physical fact is that any analytical solution that deviates away from the constraints as imposed by our temporal $(t>0)$ universe is not a physically real solution. But this does not mean that the virtual mathematical empty space and Newtonian space are useless. On the contrary, they have been the cornerstones of physics, giving us the wisdom of science, from virtual space, Newtonian space, to temporal universe as presented in Figure 1(b)-(d), respectively.

There is however a difference in context between timeless $(t=0)$ space and time-independent variable Newtonian space. Timeless $(t=0)$ space means that a virtual subspace existed with no time (i.e., $\mathrm{t}=0$ ) where time is "not" a variable, while Newtonian space means that a space existed at any time where time is an independent variable. And again, temporal $(t>0)$ space means that a space coexisted with time, where time is a constant forward variable and its velocity is already settled by the speed of light.

\subsection{Virtual mathematical spaces}

Since virtual space has caused fictitious solution in science, I will take this opportunity to show what a mathematical virtual space is, as depicted in Figure 2. Firstly, theoretical physicists are applied mathematicians; they can draw or implant coordinate systems within an empty space they wish, despite the model being physically unrealizable.

In Figure 2(a), we see that a singularity approximated atomic model is embedded within an empty subspace, which has no dimension, no size, and no time. The difference of Figure 2(b) is that there is a virtual coordinate system that has been added in, by particle physicists, since physicists are mathematicians.

Once the coordinate system is implanted, mathematically speaking, dimension as well the sizes for all the subatomic particles cannot be ignored. But for simplicity, we can ignore the size for the time being, but not the separation between particles, since distance is time and time is distance. I have also found that with the coordinate system, we have "inadvertently" assumed time is an "independent variable" as contrasted with temporal space, where time is a "dependent" variable.

We note that since the beginning of science, we have virtually used these two mathematical paradigms for analyses, not knowing the empty space that we have used is "not" a physically realizable subspace. 


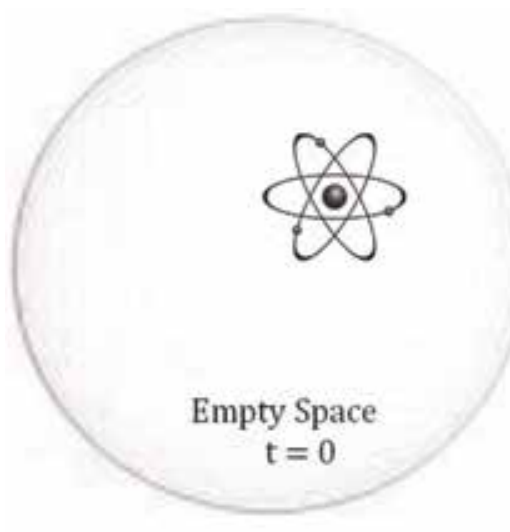

(a)

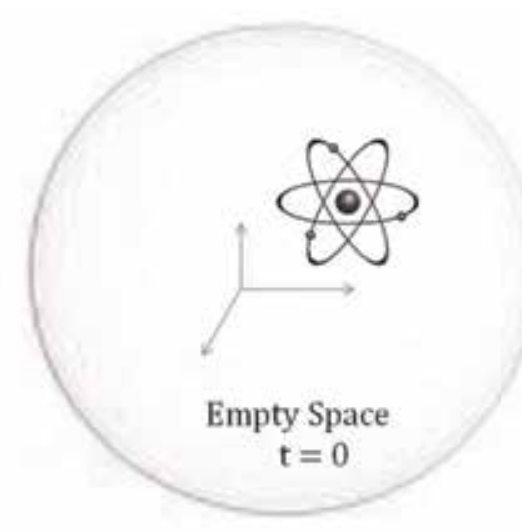

(b)

Figure 2.

A set of atomic models embedded within virtual empty subspaces (a) shows a singularity approximated atomic model is situated within an empty space, which has no coordinate system, and (b) shows an atomic model is embedded within an empty space that has a coordinate system drawn into it.

In subsequent sections, I will show severe adverse consequences have been done in the theoretical physics, for which I will show that many of their solutions and conjectures "only" exist within a timeless $(t=0)$ virtual space, which does not exist within our temporal $(t>0)$ universe.

\subsection{Timeless $(t=0)$ solution}

As from my knowledge, theoretical physics was built from a mathematical empty subspace, in which we had assumed the deep space within our universe is absolutely empty, which includes time. Since we have had experienced the existence of gravitational fields and electromagnetic wave, it tells us the deep space within our universe is "not" an empty space. In fact every subspace within our universe is temporal ( $t>0)$, which includes substances not the particle like.

Yet, emptiness within the deep space is still lingering, which causes serious problems as science moves on to a finer scale of particle size and higher level of abstraction, such as quantum mechanics, particle physics, and cosmology. Over a century of modern physics, yet we have "inadvertently" been using the same mathematical virtual paradigm for solution, not knowing the shadow background (e.g., a piece of white scratch paper) model is timeless $(t=0)$, although "all" the laws of science practically were developed from an empty virtual space.

Let me illustrate what a timeless $(t=0)$ physical law is, for which I take two of the most famous equations in modern physics, Einstein's energy equation [7] and the Schrödinger equation [2, 7], for examples. One has been used for the creation of our temporal $(t>0)$ universe $[5,9]$, and the other has many practical applications in high-speed Internet communication and computing as given by, respectively,

$$
\mathrm{E}=\mathrm{mc}^{2}
$$

where $\mathrm{m}$ is the rest mass and $\mathrm{c}$ is the velocity of light.

$$
\frac{\partial^{2} \psi}{\partial x^{2}}+\frac{8 \pi^{2} \mathrm{~m}}{h^{2}}(E-V) \psi=0
$$


where $\psi$ is the Schrödinger wave function (or Eigen function), $m$ is the mass, $E$ is the energy, $V$ is the potential energy, and $h$ is the Planck 's constant.

In view of these equations, we see that they are timeless $(t=0)$ since they are not time domain equations. In addition we also see that these equations are pointsingularity approximated; dimensionless and have no coordinate. But, a question may be asked: Why are these equations timeless $(t=0)$ ? Apparently these equations were derived from a virtual subspace, which has no time $(t=0)$.

By the way, practically all the fundamental laws and principles of science were developed on a piece of scratch paper (or on a blackboard), as shown in Figure 3.

We see a couple of equations with an atomic model drawn in it. This is a typical example of solving a particle physics problem: a scratch paper, a pencil, a model, and mathematics. Yet, if I tell you that solution as will be obtained from this configuration will be mathematically correct, but is physically "wrong," would you believe me? And this is precisely the major wrong part as I will discuss in this article.

Since we have never in our wildest nightmare that the background of a piece of scratch paper represents an empty timeless $(t=0)$ space; it is a virtual subspace that have been "inadvertently" using since the beginning of science. For which we see that; practically all the laws of physical were developed on the top of a piece of scratch paper that represents a virtual empty subspace. And I have recently found it is not a real physical subspace that supposes to be used within our universe.

In fact, practically all the laws of science were obtained from this virtual subspace.

In the following, I will show consequences that have been from the use of a piece of scratch paper. Let me start with our universe, which is a time-space interdependent space as described by the following symbolic representation $[5,9]$ :

$$
\mathrm{f}[\mathrm{r}(\mathrm{t})], \mathrm{t}>0 \text { and } \mathrm{r}=\mathrm{c} \cdot \mathrm{t}
$$

where $\mathrm{r}$ is the radius of a subspace, $\mathrm{c}$ is the velocity of light, and $\mathrm{t}>0$ denotes time as a forward dependent variable moving at a constant speed, for which we

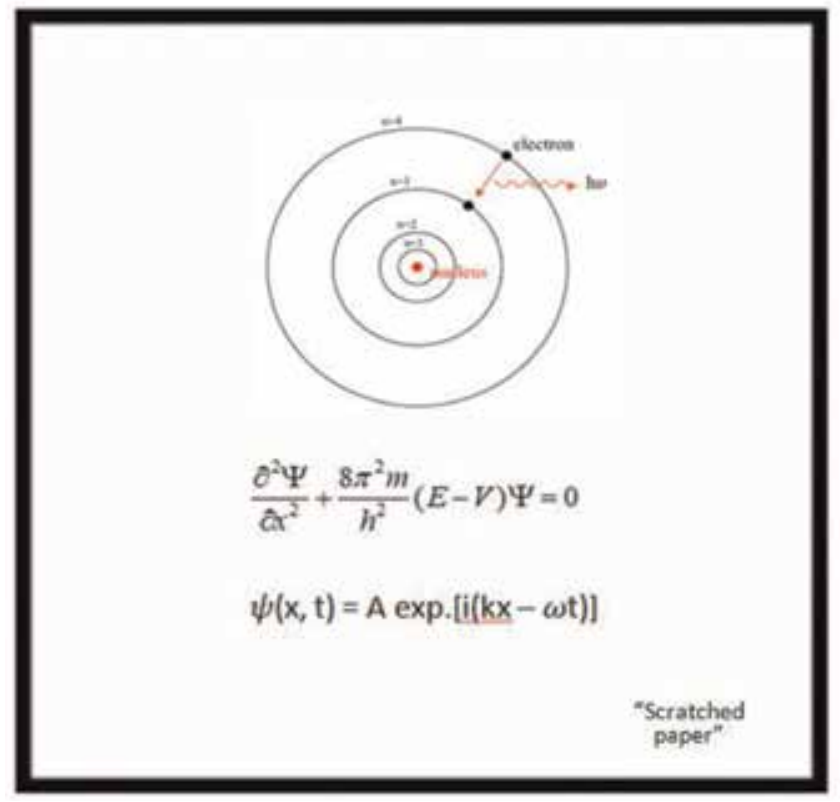

Figure 3.

A sample piece of "scratch paper" shows an atomic model with a couple of equations in it. 
see that any mathematical solution (or principle) has to comply with our universal boundary condition: causality and dimensionality. Otherwise the solution (or principle) cannot be directly implemented within our temporal $(t>0)$ universe.

In view of Eq. (1) and Eq. (2), we see that they are timeless $(t=0)$ equations; strictly speaking they cannot be directly applied into our temporal $(t>0)$ universe, unless a temporal component is introduced with these equations. For example, let us take the timeless Einstein energy equation as our example. If the equation is appropriately converted into a partial differential form as given by $[5,9]$

$$
\frac{\partial E}{\partial \mathrm{t}}=-c^{2} \frac{\partial m}{\partial \mathrm{t}}=\nabla \cdot \mathrm{S}, \mathrm{t}>0
$$

we see that the equation has transformed from a timeless $(t=0)$ dimensionless equation into a time-dependent equation with spatial representation, where a partial derivative of energy with respect to time $\left(\frac{\partial E}{\partial t}\right)$ is the rate of energy increase, the term $\left(\frac{\partial m}{\partial \mathrm{t}}\right)$ represents corresponding rate of reduction in mass, $\nabla$ represents the divergent operator, - denotes the dot product operation, $S$ is an energy vector, and $\mathrm{t}>0$ denotes time as a dependent forward variable after excitation $\mathrm{t}=0$.

From Eq. (4) we see that a dimensionless and timeless equation has been transformed into a time variable function that can be applied directly within our temporal $(t>0)$ universe. Notice that an equation is not just a symbolic representation, it is also a description, in which we can visualize that the converted energy diverges at speed of light into a dimensional space as time moves forward. This is precisely how our universe was created, in which we see that "every" subspace, within our temporal $(t>0)$ universe, can be described by the following expression as given by

$$
\nabla \cdot S=\mathrm{f}[\mathrm{r}(\mathrm{t})]=\mathrm{f}[\mathrm{x}(\mathrm{t}), \mathrm{y}(\mathrm{t}), \mathrm{z}(\mathrm{t})], \mathrm{t}>0
$$

where $\mathrm{r}$ is the radius of a spherical subspace and $[\mathrm{x}(\mathrm{t}), \mathrm{y}(\mathrm{t}), \mathrm{z}(\mathrm{t})]$ represents a rectangular coordinate system. Note that any particle regardless of their size, very large or very small, can be represented by Eq. (5), which includes all the elementary particles, in which we see that all particles are temporal $(t>0)$ particles that include substances, not particle size. Nevertheless Eq. (5) can be further extended as given by $[5,10]$

$$
[\nabla \cdot S(v)]=-\frac{\partial}{\partial t}\left[\frac{1}{2} \epsilon o E^{2}(v)+\frac{1}{2} \mu \mathrm{o} H^{2}(v)\right], \mathrm{t}>0
$$

where $\left(\epsilon_{\mathrm{o}}, \mu_{\mathrm{o}}\right)$ denotes the permittivity and permeability medium within the free space; $(\mathrm{E}, \mathrm{H})$ represent the electric and magnetic field vectors, respectively; and $v$ is the frequency of the electromagnetic wave. From this extended representation, we see that the boundary of our universe is expanding at a speed of light within an even larger space well beyond our current observation, as depicted in Figure 4.

Since the speed of electromagnetic wave is limited by $1 /[\epsilon \mu)]^{1 / 2}$, we see that our universe is "not empty," which includes the space beyond our universal boundary; otherwise our universe will "not" be a bounded subspace. The non-emptiness universal space is an interesting aspect of a greater universe, as I will discuss briefly when we meet at the Big Bang creation.

As we have accepted the deep space of our universe is non-empty, any excitation within our temporal $(t>0)$ universe "cannot" be instant $(t=0)$ responded, but it 


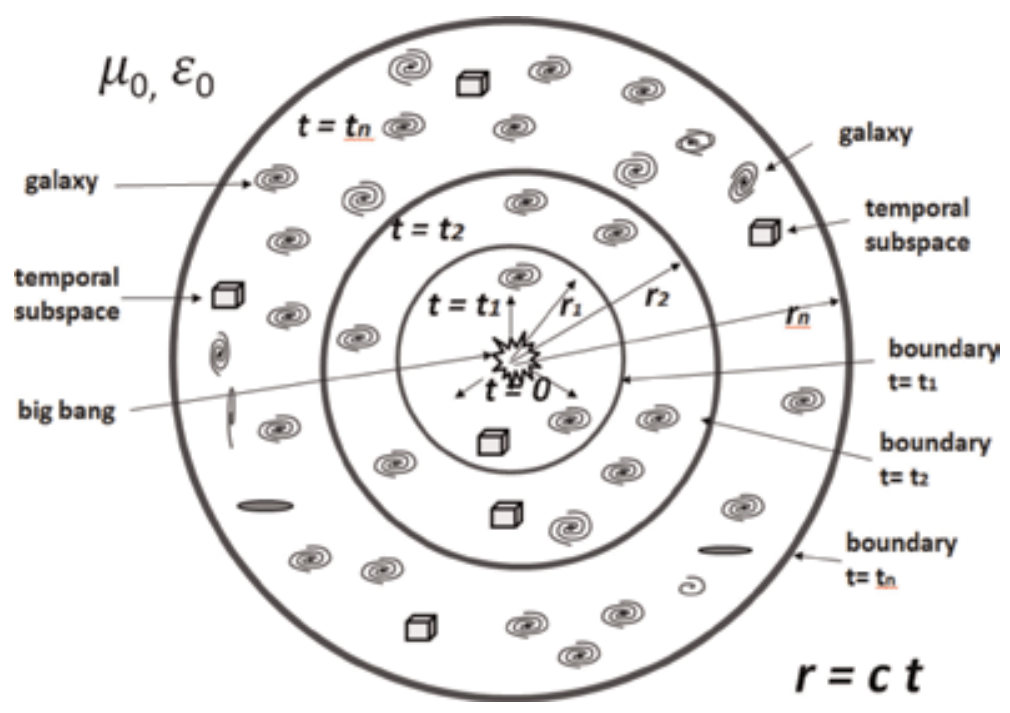

Figure 4.

A composite temporal space universe diagrams. $r=c t, r$ is the radius of our universe, $t$ is time, $c$ is the velocity of light, and $\epsilon_{o}$ and $\mu_{o}$ are the permittivity and permeability mediums within the deep space.

will be responded at a later instance (i.e., $t>0$ ). This is the well-known causality condition, a well-accepted principle in science.

Moreover, Eq. (6) shows that time and subspace are mutually coexisting within our universe by which time and space are interdependent. That is, time is a "dependent" variable with respect to the existence of the subspace, and space is a dependent substance with respect to time, in which we see that, within our universe, time is space and space is time, for which we see that it may be possible to transform a timeless $(t=0)$ equation into a time domain equation, to comply with the causality $(t>0)$ condition of our universe, as will be shown in the following:

For example, Figure 5 shows a timeless solution which can be transformed and reconfigured into a temporal $(t>0)$ domain solution. Let me assume a Fourier domain solution $F(\omega)$ is depicted in Figure 5(a), where $\omega$ is an angular frequency variable, in which we see that it is a timeless $(t=0)$ equation (i.e., not a time domain equation) which cannot be used within our temporal universe. By simply inverse-transforming $F(\omega)$ into a time-domain solution [i.e., $f(t)$ ] shown in Figure 5(b), we see that it is still not a physical realizable solution, since a portion of $\mathrm{f}(\mathrm{t})$ exists in the negative time domain (i.e., $t<0$ ). However if we add a negative linear phase distribution [i.e., exp. $(-\mathrm{id} \omega)]$ with $\mathrm{F}(\omega)$, together we have $[\mathrm{F}(\omega)$ exp. $(-\mathrm{id} \omega)]$; again it is still not a time domain solution. If this complex Fourier domain solution is inversely transformed into a time domain equation of $f(t-d)$ as depicted in Figure 5(f), we see that $f(t-d)$ exists only within the positive time domain which can be directly applied within our temporal subspace, since it satisfies the causality ( $t>0)$ condition of our universe.

In this example, we have shown, in principle, it is possible to reconfigure a temporal solution to comply with the causality $(t>0)$ condition of our universe. But there is always a price to pay (i.e., in time $\mathrm{d}$ ); by appropriately delaying a nonphysical realizable time domain solution, it is possible to make a time domain solution causal (i.e., $\mathrm{t}>0$ ).

This example also shows an important aspect within our temporal universe; we cannot get something from nothing; there is always a price to pay, an amount of energy with a section of time (i.e., $\Delta \mathrm{E}$ and $\Delta \mathrm{t}$ ). This means that every subspace within our temporal $(t>0)$ universe responds after the excitation. In other words 


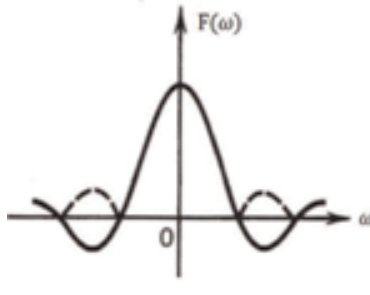

(a)

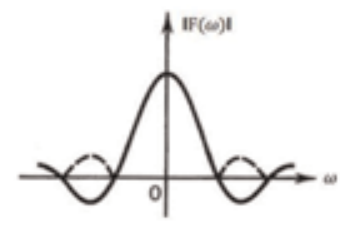

(c)

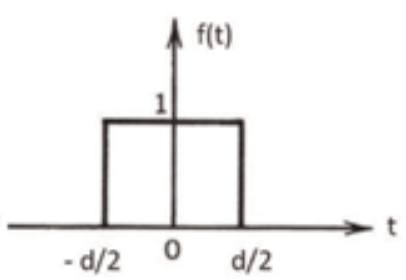

(b)

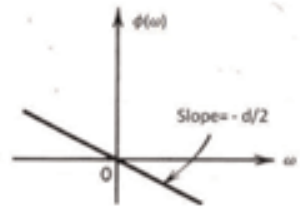

(d)

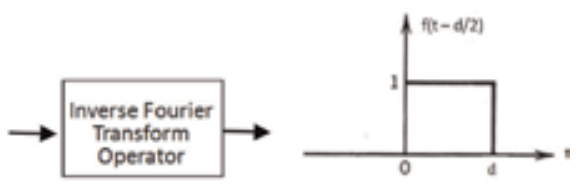

(e)

Figure 5 .

(a) A Fourier domain solution; (b) the corresponding time domain solution; (c) magnitude of a Fourier distribution; $(d)$ a Fourier domain linear phase factor; $(e)$ an inverse Fourier transformer; and $(f)$ the corresponding time domain solution existing in the time domain (i.e., $t>0$ ).

temporal $(t>0)$ space behaves as a passive time-dependent system; it responds after the excitation but neither instant (i.e., at $t=0$ ) nor before time (i.e., $t<0$ ).

The essence of this illustration is that it tells us, in principle, is possible to transform an arbitrary solution to become a time domain solution. Any analytic solution as obtained from those fancy mathematics (e.g., Hilbert space, topological space, and others) in principle can be converted first into a time domain solution and then reconfigures it into a temporal $(t>0)$ solution to satisfy the causality $(t>0)$ condition of our universe.

Again, practically all the fundamental laws of physics are timeless $(t=0)$ and singularity approximated. In principle a causality $(t>0)$ constraint can be added with those laws, as to signify the imposition upon time is a forward dependent variable (i.e., $t>0$ ). A sample set of well-known equations that can be constrained by $t>0$ is given by

$$
\begin{gathered}
\nabla \times E=-\frac{\partial B}{\partial t}, t>0 \\
\nabla \times B=\mu_{0} J+\mu_{0} \varepsilon_{0} \frac{\partial E}{\partial t}, t>0 \\
\frac{\partial \varepsilon}{\partial t}=-c^{2} \frac{\partial m}{\partial t}, t>0 \\
\psi(\mathrm{x}, \mathrm{t})=\exp \cdot[\mathrm{i}(\mathrm{kx}-\omega \mathrm{t})], \mathrm{t}>0
\end{gathered}
$$

in which we see these equations as well as their solutions are and will be subjected to the causality $(t>0)$ constraint. By reconfiguring their solutions to comply with the causality ( $t>0)$ condition, all solutions can be applied within our universe. For example, without the causality (i.e., $t>0$ ) constraint, wave equation of Eq. (10) is not a physical realizable time domain solution that can be directly applied into our universe, although it is a time-domain solution. With the imposition of $\mathrm{t}>0$, her solution can be shown approximately as given by [10]: 


$$
\psi\left[\left(\mathrm{t}-\mathrm{t}_{0}\right)=\exp \cdot\left[-\alpha\left(\mathrm{t}-\mathrm{t}_{0}\right)^{2} \cos [\omega(\mathrm{t})], \mathrm{t}-\mathrm{t}_{0}>0\right.\right.
$$

in which we see that the wave equation existed within the positive time domain, as long as $t-t_{0}>0$ and $t_{0}$ is a time delay factor.

\section{Virtual paradigm}

Science is based at different levels of physical abstraction, in which the foundation of theoretical physics is supported by mathematics with physical realizable paradigms. As earlier as the discovery of gravitational field, to Newtonian mechanics, to statistical mechanics, to electromagnetic field, to relativistic theory, to particle physics, and to quantum mechanics, each level of physical discovery was based on an assumed physical realizable paradigm, in which their analytical solutions were assumed physically real, with a high degree of certainty.

But as science progresses, the demand for more-defined physical paradigms is needed. The fact is we have "inadvertently" overlooked and continuously used an old virtual subspace which is no longer viable for sophisticated modern physics. Although irrational and fictitious solutions emerged, which have been pointed to us that something is wrong with our solutions, ambition and fantasy have driven us to quick success, for overlooking what is wrong with the theoretical analysis, since theoretical physics was and still is the core of modern physics. But the problem of theoretical physicists is that they have used sophisticated mathematics to substitute the physical reality, inadvertently or intentionally not finding out what is wrong with their irrational solutions, which have been known as spooky solutions.

In the following I will show a few typical examples without quotation, since there are a bunch of evidences which are difficult to single out for references, for which the readers can find those materials either published in various scientific journals or in the YouTube links as well as in various social media posted by wellknown theoretical physicists from prestigious universities and national research institutes that include some Nobel laureates in physics.

\section{Big Bang hypothesis}

Although Big Bang creation is a well-accepted hypothesis, it was assumed the explosion started from a singularity explosion within an "empty space" as depicted in Figure 5(a).

Since empty space cannot have non-empty subspace in it, we see that paradigm of Figure 6(a) is "not" a physical realizable model. Strictly speaking any nonphysical realizable paradigm as Figure 6(a) should not be used; otherwise unsupported virtual solution may emerge such as cyclic universe creation, single universe theory, and others.

Even though we assumed Big Bang explosion occurs within an empty space, as cosmologists often do, the velocity of electromagnetic radiation will be infinitely large, by virtue of electromagnetic wave velocity as given by

$$
\mathrm{v}=1 /(\epsilon \mu)^{1 / 2}
$$

where $(\epsilon, \mu)$ are the permittivity and permeability medium. Within an empty space, we see that $\epsilon=0$ and $\mu=0$. 


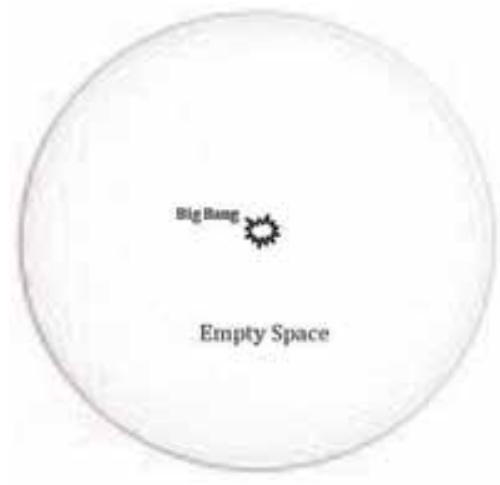

(a)

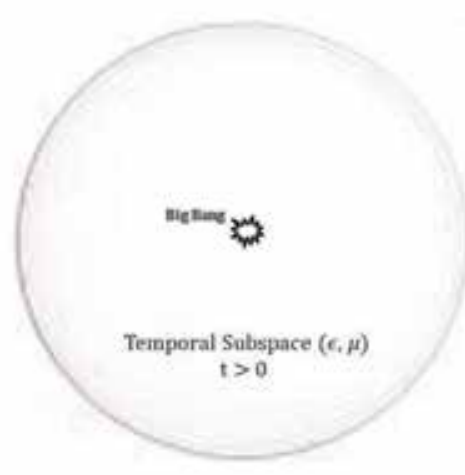

(b)

Figure 6.

(a) Big Bang explosion started within an empty space, a generally accepted paradigm. (b) Big Bang explosion within a temporal $(t>0)$ subspace; $(\epsilon, \mu)$ represents the space which already has substance in it.

On the other hand, if the solution comes out from a Big Bang explosion situated within a temporal $(t>0)$ space, as depicted in Figure 6(b), we see that the explosion started within a non-empty subspace where time has already coexisted with the "media"(e.g., $\epsilon$ and $\mu$ ) within the subspace, in which we see that our universe is a "bounded" temporal ( $t>0)$ subspace and her boundary is expanding at the speed of light, which is consistent with the observation reported by the Hubble Space Telescope [11].

\section{Timeless quantum world}

Two of the most important pillars in modern physics must be Einstein's relativity theory [7] and Schrodinger's quantum mechanics [2], in which one is dealing with very large objects and the other is dealing with very small particles. Yet they were connected by means of Heisenberg's uncertainty principle [12]; every subspace within our temporal $(\mathrm{t}>0)$ universe is limited by energy (i.e., $\Delta \mathrm{E}$ ) and time (i.e., $\Delta t$ ), which is equaled to a "quantum unit" of Heisenberg's principle [13] as given by

$$
\Delta \mathrm{E} \cdot \Delta \mathrm{t}=\mathrm{h}
$$

where $\mathrm{h}$ is the Planck's constant.

And each quantum unit is equivalent to an information cell, as shown by Dennis Gabor in 1946 [14], in which it tells us that every bit of information takes an amount of energy $(\Delta \mathrm{E})$ and section of time $(\Delta \mathrm{t})$ to produce, to transmit, to store, and to destroy, and it is "not" free (i.e., in terms of $\Delta \mathrm{E}$ and $\Delta \mathrm{t}$ ). In other words; within our universe everything has a price tag; namely $\Delta \mathrm{E}$ and $\Delta \mathrm{t}$.

Since Schrodinger's quantum mechanics was constructed within a timeless $(t=0)$ subspace, his whole quantum world is timeless [15]. And this is the "same error" that all of us, past and present, have committed, by a paradigm which was drawn into a scratch paper and not knowing it actually represents a timeless $(t=0)$ subspace.

In view of Figure 7(a), I sincerely "believed" that Schrödinger was not aware the background of a scratch paper presented as a virtual empty subspace; otherwise he would not have had the paradox of his cat. 


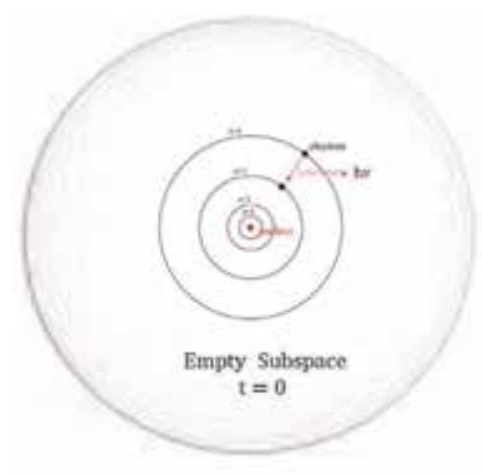

(a)

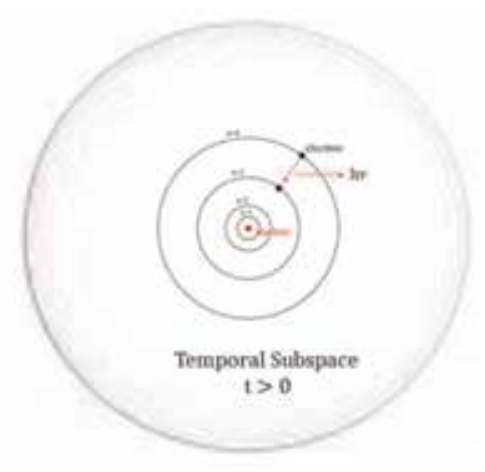

(b)

Figure 7.

Physical realizable paradigm (a) shows a Bohr atom situated within an empty space, which is "not" a physical realizable model, and $(b)$ shows a Bohr atom situated within a temporal subspace, which is a physical realizable model.

Why we have missed the timeless $(t=0)$ subspace paradigm issue is very "natural," since we are all human, imperfect and simple as it is. And we have "inadvertently" treated the shadow background of a scratch paper as a physical space, and it has never been in our minds that the background represents a virtual empty space. And this is precisely the reason that Schrödinger's quantum mechanics did.

Nevertheless, it is possible to build a "temporal $(t>0)$ " quantum machine [10] similar to the one that Schrödinger has built. Instead, the quantum machine is built with a Bohr atomic embedded within a temporal ( $t>0)$ subspace, as depicted in Figure $7(b)$, in which we see that any analytical solution that emerges from this physical realizable paradigm will be temporal $(t>0)$, for which I note that if one is searching a new particle in a timeless $(t=0)$ quantum world, it is "very likely" the newly discovered (i.e., analytical) particle is timeless $(t=0)$, since every particle is also a temporal particle within our universe, no matter how small it is; otherwise the particle cannot exist within our temporal universe. In case one "insists" that a timeless particle can coexist within our universe, it is equivalent of searching for a timeless $(t=0)$ particle within our temporal universe.

\section{Granular universe}

As from the particle physics perspective, every substance within our universe is built by particles, of which our universe is compacted with particles, for which we see that our universe is granular instead of smooth and continuous. And it is a very convincing argument from a particle physicist's point of view: our universe is not continuous and smooth.

Since particle is temporal $(\mathrm{t}>0)$ no matter how small they are, empty space cannot coexist within a temporal $(t>0)$ space, in which we see that there should be "temporal" substances between particles. And this is precisely why there are existent temporal substances throughout the entire universe, beyond particle forms. Otherwise gravitation fields, electric fields, magnetic fields, as well electromagnetic waves cannot exist within our universe.

With all these physical evidences, we see that there is a new branch of physics beyond the particle physics that is waiting for us to explore, for which the "micro limit" of physics should not have to be limited to the particle physics point of view, 
in which we see that time is not as granular as predicted by particle physicists. As we accepted particle and time coexist, we see that every particle within our universe has the same instance of time with the same time speed but has a relativistic time between particles, in which time is a dependent variable with respect to particle and it is as smooth and continuous.

\subsection{Symmetric principle}

Symmetric principle has been used in theoretical physics for searching new particles and others, since the dawn of modern physics. Mathematically speaking those imaged properties of particles exist, but they are from an abstract mathematical standpoint.

Symmetric principle is based on a virtual empty space where time is treated as an independent variable as a Newtonian subspace. And this is precisely why the symmetric principle of science behaves as a mirror perception, such as positive versus negative or as groups in group theory, for example, positive time versus negative time, positive energy versus negative energy, matter versus anti-matter, and others. It is physical real versus mathematical virtual. But the fact is that all the mirror images such as negative time, negative energy, and anti-matter do not exist within our temporal $(t>0)$ universe. The fact is that those conjectures were derived from a mathematical or a Newtonian space standpoint; which is not existed within our temporal $(t>0)$ universe that has had or has had not!

Since time and space coexist, matter (i.e., subspace) is time, and time is matter. Without time we have no matter, and without matter it has no time, in which we see that there is an "asymmetric principle" in science with respect to physical real versus mathematical virtual, instead of the mirror image of mathematics.

Samples of asymmetric principle are:

Have time $(\mathrm{t}>0)$ vs. no time $(\mathrm{t}=0)$

Having energy vs. no energy

Having matter vs. no matter

Physical real vs. virtually fictitious

Therefore we see that searching for any new particle, it is more reasonable to use the "asymmetric principle" instead of symmetric principle. It will be more "likely" found within our physical world, since timeless particles do not exist within our temporal $(t>0)$ universe.

\subsection{Curving time-space}

Within our temporal ( $t>0$ ) universe, every subspace takes an amount of energy $\Delta \mathrm{E}$ and a section of time $\Delta \mathrm{t}$ to create, and it is not free, for which time is subspace and subspace is time, and we see that time is a "dependent" variable with the existence of space. Since we are still having a vague idea of interaction between gravity with time and knowing that gravitational field is produced by masses, gravitational field has to be embedded in a non-empty space which coexists with time. It is therefore "incorrect" to assume time as an "independent" variable, as we often do, for which I have a hard time to accept curving time-space dynamics, as based on a virtual mathematical space.

Since speed of time is settled by the velocity of light as our universe was created by a Big Bang explosion (a well-accepted paradigm) from the theory of relativity $[5,9]$, we have shown time is a dependent variable, instead of an independent variable, as within a Newtonian subspace, where time traveling is possible.

And this is the reason why space "cannot" be curved by time, since time is a "dependent" variable, of which we see that time is "physical real" since time and 
subspace coexist, in which we see that time is certainly "not" an illusion as some scientists claimed.

\subsection{Entropy and information}

Aside from the virtual empty space paradigm, there are some serious mistakes that have been made on entropy theory of information by some theoretical physicists. Since information theory was developed by a group of mathematically oriented engineers, it was hardly appreciated by the physicists until it is connected with Boltzmann's second law of thermodynamics as given by

$$
\mathrm{I}=\log _{2} \mathrm{~N} \text { bits }
$$

and

$$
\mathrm{S}=\mathrm{k} \ln \mathrm{N} \text { joules per Kelvin }
$$

where $\mathrm{k}$ is the Boltzmann constant, in which we see that information and entropy can be exchanged or traded, as given by the following symbolic representation:

$$
\mathrm{I} \leftrightarrow \mathrm{S}
$$

Without this connection, information would be very difficult to be applied in physics, since entropy is a well-accepted quantity in science.

However, the relationship of Eq. (16) does not mean that quantity of entropy (or equivalent amount of information in bits) is equaled to the information. It is the "cost" in bits (or equivalent amount of entropy in joules/kelvin) needed to generate the information, for example, a book of 1000 bits of information content (i.e., the cost), which means that there are $2^{1000}$ possible copies of books having the same 1000 bits of information. There are also many objects available having the same bits of information, but not books.

As for quantum entanglement communication, it seems to me they have missed the essence of information transmission. For "efficient" information transmission, the sender (i.e., information source) is required to provide a highest information content (i.e., equip-probable state) of the source. For example, the more equalprobable or "uncertain" the ensemble signal is provided by the sender, the higher the information content from the source. For example, information content provided by the source (i.e., the sender) is given by

$$
\mathrm{I}=-\log _{2} \mathrm{p}\left(\mathrm{a}_{\mathrm{i}}\right)
$$

where $\mathrm{p}\left(\mathrm{a}_{\mathrm{i}}\right)$ is the probability of an event $\mathrm{a}_{\mathrm{i}}$ from the source (i.e., sender) provider ensembles $A=\left\{a_{i}\right\}, I=1,2, \ldots, N$, in which we see that the largest information content provided by the source is $\mathrm{P}=1 / \mathrm{N}$ (i.e., equal probability state).

As for a "binary" information source (i.e., 0,1 ), for $\mathrm{N}=2$ it is the maximum entropy-coded binary source as given by

$$
\mathrm{I}=-\log _{2} \mathrm{p}(1 / 2)=1 \mathrm{bit}
$$

in which we see that binary source information is the "lowest" information capacity for transmissions per unit time $\Delta t$, if we used digital transmission. Yet, the major advantage for using binary-digital transmission is for noise immunization, 
such that the corrupted digital signal (e.g., by noise) can be refreshed, since digital signal can be repeated. For example, a compact disk (i.e., CD) can copy for thousands of time, and we have experienced that the latest copy is just as good as the original copy, while an analog magnetic tape cannot.

One of the important aspects for information transmission is that "reliable" information can be transmitted, such that information can be reached to the receiver with high degree of certainty. Let me take two key equations from information theory, mutual information through a "passive additive noise channel" as given by [16]

$$
\mathrm{I}(\mathrm{A} ; \mathrm{B})=\mathrm{H}(\mathrm{A})-\mathrm{H}(\mathrm{A} / \mathrm{B})
$$

and

$$
\mathrm{I}(\mathrm{A} ; \mathrm{B})=\mathrm{H}(\mathrm{B})-\mathrm{H}(\mathrm{B} / \mathrm{A})
$$

where $\mathrm{H}(\mathrm{A})$ is the information provided by the sender, $\mathrm{H}(\mathrm{A} / \mathrm{B})$ is the information loss (or equivocation) through transmission due to noise, $H(B)$ is the information received by the receiver, and $\mathrm{H}(\mathrm{B} / \mathrm{A})$ is the noise entropy of channel.

However there is a basic distinction between these two equations: one is for "reliable" information transmission to the receiver, and the other is for "retrievable" information from the source (i.e., sender). Although both equations represent the mutual information transmission between sender and receiver; but the objective for using Eq. (19) is that "sender" "carries" an "active" role in achieving a reliable information transmission to the receiver, while using Eq. (20) is that "receiver" plays a more active role to deal with information that has been received. In which we see that; as for "reliable" information transmission is to increase the signal-tonoise ratio (i.e., $\Delta \mathrm{E}$ ) at the transmitting end. While for "retrievable" information after it has been received. In other words, one is to be sure information will be reached to the receiver "before" information is transmitted, and the other is to retrieve the information "after" information has been received.

In communication theory, basically we have two types communication strategies: by Norbert Wiener [17, 18] and by Claude Shannon [19]. However there is a major distinction between them; Wiener's communication strategy is that, if the information is corrupted through transmission, it may be recovered at the receiving end, but with a "cost," mostly at the receiving end, while Shannon's communication strategy carries a step further by encoding the information before it is transmitted, such that information can be "reliably" transmitted, also with a "cost" but mostly at the transmitting end. In view of the Wiener and Shannon information transmission strategies, mutual information transfer of Eq. (19) is kind of Shannon type, while Eq. (20) is for Wiener type, in which we see that "reliable" information transmission is basically controlled by the sender. It is to "minimize" the noise entropy $\mathrm{H}(\mathrm{A} / \mathrm{B})$ (or equivocation) of the channel, as shown by

$$
\mathrm{I}(\mathrm{A} ; \mathrm{B}) \approx \mathrm{H}(\mathrm{A})
$$

One simple way to do it is by increasing the signal-to-noise ratio, with a "cost" of higher signal energy (i.e., $\Delta \mathrm{E}$ ).

On the other hand, to recover the transmitted information is to "maximize" $\mathrm{H}$ (B/A) (the channel noise). Since the entropy $\mathrm{H}(\mathrm{B})$ at the receiving end is larger than the entropy at the sending end, that is, $\mathrm{H}(\mathrm{B})>\mathrm{H}(\mathrm{A})$, we have

$$
\mathrm{I}(\mathrm{A} ; \mathrm{B})=\mathrm{H}(\mathrm{B})-\mathrm{H}(\mathrm{B} / \mathrm{A}) \approx \mathrm{H}(\mathrm{A})
$$


Equation (22) essentially shows us that information can be "recovered" after being received, again with a price: $\Delta \mathrm{E}$ and $\Delta \mathrm{t}$. In view of these strategies, we see that the cost paid for using Wiener type for information transmission is "much higher" than the Shannon type; aside from the cost of higher energy $\Delta \mathrm{E}$, it needs an extra amount of time $\Delta t$ for "post processing." Thus we see that Wiener communication strategy is effective for a "noncooperating" sender, for example, applied to radar detection and others. On the other hand, Shannon type provides a more reliable information transmission, by simply increasing the signal-to-noise ratio so that every bit of information can be "reliably transmitted" to the receiver.

Therefore, we see that quantum entanglement communication is basically using Wiener communication strategy. The price will be "much higher and very inefficient," such as post processing. And it is "illogical" to require the received signal to be "more equivocal" (i.e., uncertain); the information recovery is better if it can be received at the receiving end, in which quantum entanglement communication is designed for extracting information as Wiener-type communication. However it is "not" the purpose for reliable information-transmission of Shannon.

\subsection{Mathematics and physics}

In view of all preceding evidences, we see that theoretical physics is mathematics, but mathematics is not physics since physics has to be physically real and should not be virtual as mathematics is. In other words, any analytical solution as obtained from any theoretical analysis has to comply with the boundary condition of our universe: causality (i.e., $\mathrm{t}>0$ ) and dimensionality; otherwise it is virtual as mathematics is. As we know that in mathematics; it needs to prove first that a mathematical postulation has a solution before searching for the solution. However, in theoretical physics it seems to me it does not have such a criterion, although theoretical physics is mathematics. Since now we have one criterion available, any solution emerging from theoretical analysis has to be shown "first" that it exists within the boundary condition of our universe. Otherwise it is a virtual solution as mathematics is.

When one is discussing the origin of science, it is inevitable not to talk about singularity approximation or singularity principle, in which sometime we underestimate the wisdoms of our predecessors. Practically all the laws of science are singularity approximated. Otherwise it is impossible mathematically to create a set of "simple and elegant" formulas for us to appreciate and to apply. Yet we tend to use the singularity approximated laws to interpret them as classical and deterministic, which undermines our predecessor's intelligence, in that they did not know there were approximated. And then we turn to using the singularity principle to evaluate the singularity approximated laws to obtain another singularity solution. It seems to me the ended singularity solution has the composition of two singularities' approximated results, for which I felt the solution will be even further deviated from the physical reality, aside from the nonphysical existing paradigm.

Let me pick a model for the illustration: the Bohr atomic model since we have used this model for over a century, shown in Figure 8(a).

We see it is a typical singularity approximated paradigm, no coordinate, no dimension, and no mass, and the entire Bohr atom is point singularity approximated. The only viable information provided by this model is the quantum state energy h $v$, which is also singularity approximated, since $v$ has no bandwidth. However in practice every quantum state radiation has to be band and time limited.

Although mathematics can be regarded as entropy or information provider, my question is how much additional information from $\mathrm{h} v$ Schrödinger can generate. In fact, he has had done an amazing task that nobody in quantum physics had done in the history of quantum mechanics, since all the viable information obtained by 


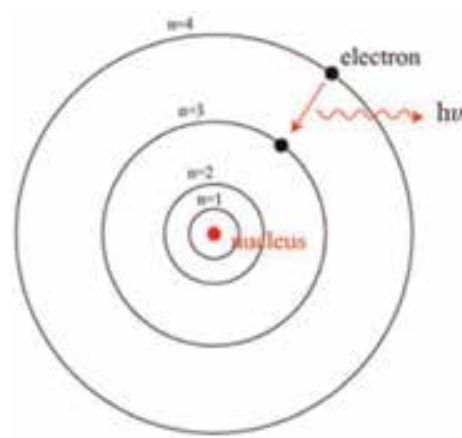

(a)

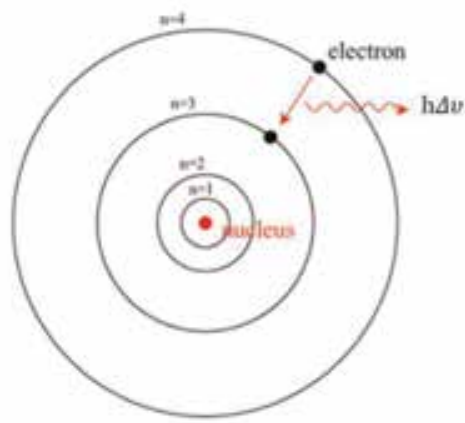

(b)

Figure 8.

(a) A conventional Bohr Atomic model and (b) the same Bohr model, except the quantum state energy is presented by a band-limited notation $h \Delta v$.

Schrödinger is based on $\mathrm{h} v$. Although singularity $\mathrm{h} v$ has led him to a timeless $(\mathrm{t}=0)$ quantum world, which was not due to mathematics, it is due to the background empty space. In other words, if he has had treated $\mathrm{h} v$ as a band-limited radiation $\mathrm{h} \Delta v$, as shown in Figure 8(b), his "timeless" fundamental principle may not have emerged, in which I have shown that small changes from singularity bandwidth (i.e., $v$ ) to band limited (i.e., $\Delta v$ ) assumption could have altered the end results tremendously.

\section{Timeless and temporal}

Let me show a system analysis to illustrate the dynamic behavior when a timeless $(t=0)$ superimposing of wavelets (or particles) is plunging into a temporal $(t>0)$ subspace. For simplicity we assumed a set of two time-limited quantum state wavelets (or two particles) are plunging into a timeless $(t=0)$ space first, and then its output is submerging within a temporal $(t>0)$ subspace, as depicted in

Figure 9.

Since empty space of Figure $\mathbf{9 ( b )}$ is timeless, we see what its response does to the particles; all collapse at $\mathrm{t}=0$ within an empty space, which shows the behavior of superposition principle dose to particles. In other words, within a timeless environment all things existed at $\mathrm{t}=0$, and all things can also be found "simultaneously and instantly" anywhere within the timeless $(t=0)$ subspace.

Now, if we further plunge this timeless response into a temporal $(t>0)$ space of Figure 9(d), we see that the response shows "no sign" of preserving the original wavelet (or particles) properties.

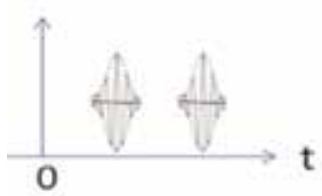

(a)

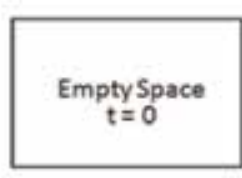

(b)

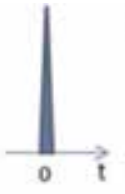

(c)

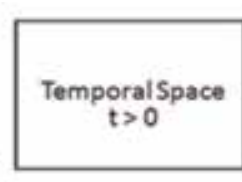

(d)

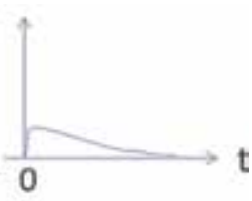

(e)

Figure 9.

A system simulation for timeless $(t=0)$ superposition within a temporal $(t>0)$ space $(a)$ shows a set of time limited wavelets (or particles) plunge into a timeless space of $(b) ;(c)$ shows the output response collapse at $t=0 ;(d)$ shows a temporal space representation; and (e) shows the corresponding response within a temporal space, in which we see that output loses all the original input personality. 


\subsection{Art of a virtual principle}

Fundamental principle of superposition is the core of Schrödinger's quantum mechanics, which is an anchor foundation for the quantum supremacy on practically everything such as quantum gravity and others as well for quantum computing and for quantum entanglement communication.

Yet, it is my responsibility to show systematically what timeless superposition principle can do to the particles, as illustrated in Figure 10.

In which we note that; the proposed particles situated within a timeless $(t=0)$ is a non-physical realizable or mathematical virtual paradigm. Let me stress that within our universe, every substance is temporal $(t>0)$ which includes all the particles no matter how small they are. Strictly speaking all particles are "temporal particles," although singularity approximated for mathematical conveniences.

Besides the nonphysical realizable issue, the quantum mechanist can also add a coordinate system within the empty space, as can be seen in Figure 10, for which we can visualize the particles' locations with respect to the coordinate system that the quantum mechanist has implanted.

Since within an empty space it has "no time" or is timeless $(t=0)$, we see that all the particles are collapsed or "superimposing instantly" at $t=0$, since time is distance and distance is time. The virtual coordinate system is assumed "as a Newtonian space in which the space is treated time as an "independent" variable. But in reality, a particle, no matter how small it is, is a "temporal $(t>0)$ particle." And this is precisely the fundamental principle of superposition, stating all particles are superimposing "instantly" at $\mathrm{t}=0$.

When we have treated a virtual empty space added with the coordinate system in it, then we have to accept distance is time and time is distance, within the virtual empty space. Then we see that every particles can exist anywhere "simultaneously" and "instantaneously" within the empty space, since it is a timeless $(t=0)$ space. And these are the "instantaneous" and "simultaneous" phenomena of Schrödinger's fundamental principle of superposition.

Nevertheless, aside from the temporal particle issue, a more crucial one is that timeless $(t=0)$ particles cannot exist within a temporal $(t>0)$ space. This is like asking all the timeless particles to perform the "simultaneous" computing and all "instantaneous" communication within our universe.

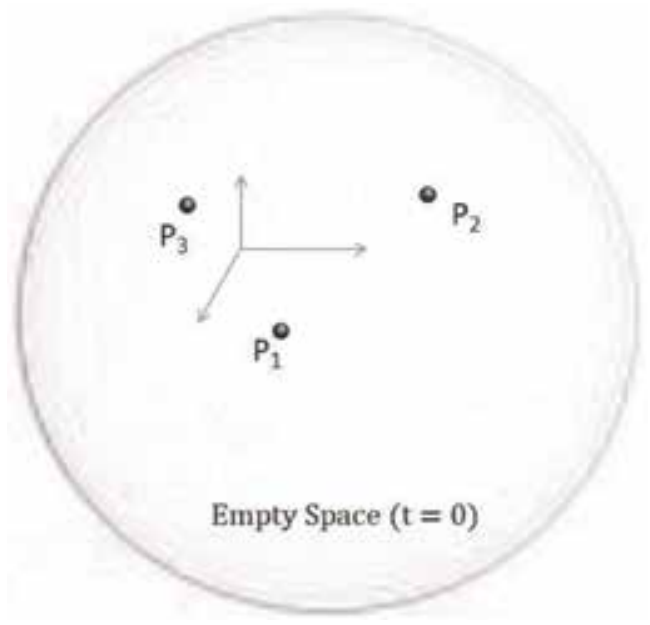

Figure 10.

Three separated particles are situated within an empty subspace in different locations. Notice it is a virtual paradigm since substance cannot exist within an empty space. 


\section{Remark}

As science progresses with time, this is the moment to answer the question that I have had asked: what is "wrong" with current theoretical physicists? In view of the preceding facts of evidences, it is apparent that the part of wrongness is "not" due to mathematics; it is due to the analytical solution. Since mathematics was not invented by the theoretical physicists, it is the underneath empty subspace from a piece or pieces of scratch papers that led us to all the fictitious solutions in that it turns out a background subspace is not a physical realizable subspace! It was never in my wildest mind that a simple piece of scratch paper used in analysis can affect that extensively! For example, all the fundamental laws of science, principles, paradoxes, and others were produced by scores of scratch papers. Yet those scratch papers also produced numbers of virtual and fictitious solutions, although "inadvertently."

In short, theoretical physicists were and still are the creators of fundamental laws of physics; it is their "responsibility" to take us back to a physical realizable world of science; otherwise we will be still trapped within a virtual timeless $(t=0)$ land of mathematics!

\section{Conclusion}

I have stressed that it is not how rigorous mathematics is, it is a physical realizable solution. Theoretical physics uses amazing mathematical analyses and singularity paradigm as well with fantastic computer animation, providing very amazing and convincing arguments. But mathematical modeling and computer animation are virtual and fictitious, of which many of their analytical solutions are not physically real. What is wrong with current theoretical physicists is that they have used a timeless $(t=0)$ paradigm for their analyses, for which their solutions do not actually exist within our temporal $(t>0)$ universe. In this article I have singled out a few typical but very important evidences to reassess our science, although I am not a physicist. In this article we have shown that timeless $(t=0)$ paradigms have been used "inadvertently" as from a piece or pieces of scratch papers for scientific analyses, which is very natural for scientific calculation, at the beginning of science. Although it has produced scores of excellent results, at the same time it has also produced many fictitious solutions that are timeless $(t=0)$.

I have also given a few typical but very important evidences that the theoretical physicists have done to physics, which includes some of the world-renowned theoretical scientists: past and present. Since theoretical physicists were and "still" are the creators of fundamental laws and principles, it is their "responsibility" to take back the physical realizable science; otherwise we will be continuingly trapped within a timeless $(t=0)$ land of mathematics.

Finally I would comment that; since all the laws and principles of science were made to revise or to be broken, I would anticipate the trend of "Temporal ( $t>0)$ Physics" is anticipated to emerge. For which I would predict more and more physically realizable analyses emerge in the first half of 21 century from the theoretical physicists; as well in the years to come. 


\section{Author details}

Francis T.S. Yu

Pennsylvanian State University, University Park, USA

*Address all correspondence to: fty1@psu.edu

\section{IntechOpen}

(C) 2020 The Author(s). Licensee IntechOpen. This chapter is distributed under the terms of the Creative Commons Attribution License (http://creativecommons.org/licenses/ by/3.0), which permits unrestricted use, distribution, and reproduction in any medium, provided the original work is properly cited. (c) BY 


\section{References}

[1] Bohr N. On the constitution of atoms and molecules. Philosophical Magazine. 1913;26(1):1-23

[2] Schrödinger E. Die Gegenwärtige situation in Der Quantenmechanik (the present situation in quantum mechanics). Naturwissenschaften. 1935; 23(48):807-812

[3] Schrödinger E. Probability relations between separated systems.

Mathematical Proceedings of the Cambridge Philosophical Society. 1936; 32(3):446-452

[4] Feynman RP, Leighton RB, Sands M. The Feynman Lectures on Physics. Cambridge, MA: Addison Wesley; 1970

[5] Yu FTS. Time: The enigma of space. Asian Journal of Physics. 2017;26(3): 143-158

[6] Yu FTS. From Relativity to Discovery of Temporal $(t>0)$ Universe, Origin of Temporal $(t>0)$ Universe: Correcting with Relativity, Entropy, Communication and Quantum Mechanics, Chapter 1. New York: CRC Press; 2019. pp. 1-26

[7] Einstein A. Relativity, the Special and General Theory. New York: Crown Publishers; 1961

[8] Belkind O. Newton's conceptual argument for absolute space. International Studies in the Philosophy of Science. 2007;21(3):271-293

[9] Yu FTS. Entropy and Information Optics: Connecting Information and Time. 2nd ed. New York: CRC Press; 2017

[10] Yu FTS. A Temporal Quantum Mechanics. Asian Journal of Physics. 2019;28(1):193-201

[11] Zimmerman R. The Universe in a Mirror: The Saga of the Hubble Space
Telescope. Princeton, NJ: Princeton Press; 2016

[12] Heisenberg W. Über den anschaulichen Inhalt der quantentheoretischen Kinematik und Mechanik. Zeitschrift für Physik. 1927; 43:172

[13] Yu FTS. Information transmission with quantum limited subspace. Asian Journal of Physics. 2018;27:1-12

[14] Gabor D. Theory of communication. Journal of the Institution of Electrical Engineers. 1946;93:429

[15] Yu FTS. Schrödinger's Cat and his Timeless $(t=0)$ Quantum World, Origin of Temporal $(t>0)$ Universe: Correcting with Relativity, Entropy, Communication and Quantum Mechanics, Chapter 5. New York: CRC Press; 2019. pp. 81-97

[16] Yu FTS. Optics and Information Theory. New York: Wiley-Interscience; 1976. p. 13

[17] Wiener N. Cybernetics. Cambridge, MA: MIT Press; 1948

[18] Wiener N. Extrapolation, Interpolation, and Smoothing of Stationary Time Series. Cambridge, MA: MIT Press; 1949

[19] Shannon CE, Weaver W. The Mathematical Theory of Communication. Urbana, IL: University of Illinois Press; 1949 



\title{
The Hot Disputes Related to the Generation of a Unified Theory Combining the Outcomes of ER and EPR Papers
}

\author{
Agaddin Mamedov
}

\begin{abstract}
We suggest a mathematical formulation which shows that gravity is the materialization of energy in space, which at zero energy input $\left(\mathbf{E}_{\mathbf{a p}}=0\right)$ in the form of entanglement of virtual space and time phases returns an event to the initial background state with restoration of the original elementary space-time frame. Based on the suggested model, the matter-antimatter relation results from the non-uniform energy conservation principle while satisfying the conservation of energy within the boundary-mapped space-time frame. The suggested approach shows that the generation of mass is the requirement of energy conservation. The mathematic model of energy conservation involves the conjugation of the dynamic local state of space and time variables with the local energy-momentum relation, which at different energy inputs can operate at the small scale of quantum physics and the large scale of relativity. The suggested theory shows that commutation of local spacetime position and energy-momentum exchange interaction is the only way for conservation of energy and momentum within a discrete space-time frame.
\end{abstract}

Keywords: Relativity, quantum mechanics, conservation of energy, origin of space-time

\section{Introduction}

The history of physics involves two great revolutionary theories: relativity and quantum physics. Quantum mechanics and general relativity operate at different space and time scales; therefore, the main problem for unification of these theories is due to the "mystery" of the scale of variables.

Recently, a new approach [1-3] for the unification of general relativity and quantum mechanics was suggested, which involves reconciling relativity's black hole space and quantum entanglement. Science News $[4,5]$ announced that a new equation ER = EPR, generated from Einstein's papers [6, 7], may provide a possible path for connection of principles of general relativity with quantum mechanics. Maldacena and Susskind [2] suggested that two distant black holes of general relativity, connected through the interior wormhole (Einstein-Rosen bridge), could be interpreted as the entangled EPR's pairs of two black holes. Maldacena and Susskind [2] showed that the formula ER = EPR (bridge between Einstein-Rosen 
(ER) wormhole [6] and Einstein-Podolsky-Rosen (EPR) entanglement [7]) can be the cornerstone of the new physics theory, connecting relativity and quantum mechanics through space-time wormholes.

Grant [5] suggested that the connection of entanglement with the space-time might help to understand the origin of space-time and its behavior at the small scales of quantum mechanics.

Siegfried [8] also discussed the ER = EPR equation as a possible approach for connection of space-time geometry of relativity with the quantum entanglement.

Van Raamsdonk [9] showed that the existence of space-time is due to the quantum entanglement in the corresponding quantum system.

Carroll suggested [10] a similar approach in accordance of which space can emerge from a quantum state. Caroll's comments on connection of space-time curvature with energy are similar to the statement of general relativity, but in this approach, origin of space connected with the quantum entanglement.

While a path to quantum gravity through the wormhole became a very hot topic for the generation of new physics, it could be very useful to return to the ER [6] and EPR [7] papers to summarize the basic principles on how the combination of outcomes from the ER and EPR papers may unify different scale interactions.

The main idea of the ER paper [6] is a presentation of the physical space by field equations where space involves two identical, equal halves, separated by the symmetry and connected by a Wormhole Bridge. The separated halves of space describe the same physical space. The idea of this approach is the application of space field equations for the description of quantum level interactions.

The ER bridge, which is also called a wormhole, denotes a shortcut connection between widely separated regions of space-time. In accordance with the hypothetical equation ER = EPR [1-3], an ER wormhole between two places of space could be considered as an entangled pair in quantum mechanics.

It is necessary to note that the ER paper [6], as was claimed by the authors, does not contain the quantum phenomena and the interaction between two identical pieces of space does not lead to the "quantization of gravity."

The EPR paper [7] gives an analysis of the basic principles of quantum mechanics such as the description of state by the wave function to predict a particle's behavior. It establishes the now well-known cornerstone of quantum mechanics that two physical quantities such as position and momentum of a particle cannot be precisely determined simultaneously and that these two quantities cannot have simultaneous reality. The authors showed that the quantum mechanical description of physical reality given by wave functions is also not complete and it is necessary to assign two different wave functions to the same reality.

It is necessary to note that the conjugation of statements of the ER [6] and EPR [7] papers does not reveal a non-hypothetical mathematical equation of a dynamical event, which may explain physical reality regardless of scale.

\section{The main principles of ER and EPR papers}

We can select important statements of these papers, which were the cornerstone [1-3] for the unification of relativity with quantum mechanics:

a. Entanglement (connection) of two equal pieces of space through the "Wormhole Bridge" of relativity theory [6].

b. The common uncertainty principle of quantum mechanics is that when the momentum of a particle is known, its coordinate has no physical reality [7]. 
We should concentrate our attention to the statement of quantum mechanical uncertainty to understand why the combination of general relativity with quantum mechanics does not generate a valid mathematical formulation, which is applicable for small- and large-scale interactions.

First, it is necessary to show that a particle may have a certain identity when the change of energy in time (conservation of energy) has commutation with the change of energy, consumed in space phase of a particle (conservation of momentum). The mathematical description of momentum may be valid only if the emerged formulation comprises residing of energy-momentum exchange interaction within boundary-mapped space-time frame.

Therefore, momentum "as the quantity of motion" alone without locality is uncertain and cannot give a proper mathematical description of this quantity in any physical theory. If momentum conservation has no commutation with the energy conservation within the space-time frame, it cannot describe an event in a proper way. However, without the space-time boundary, it is difficult to get commutation of energy-momentum conservation laws. Description of motion by a model, involving a certain local boundary of the dynamical space-time frame, generates a reference frame independent of the true momentum conservation principle.

Quantum mechanics states that position and momentum cannot have simultaneous reality, due to the application of the mathematical formulation of uncertainty, which conjugates position, independent from space-time and momentum and also independent from the action-response interaction.

The Heisenberg uncertainty principle in not a mystery of nature; rather, it is an improper mathematical formulation, which does not involve missed local spacetime position and conjugation of a local position with the applied force, leading to the change of momentum.

An event of measurement is not a simple displacement of a particle position in the abstract Hilbert space, but it is the change of space-time phases as an outcome of energy-momentum action-response interaction. Therefore, change of position of a system is not a linear transformation of the space dimension; it should describe commutation of local space-time frame with the action-response energymomentum exchange interactions.

It is necessary to show that commutation of two parameters, such as local spacetime position and energy-momentum exchange interaction, is the only way for conservation of energy and momentum within the discrete space-time frame.

The concept, describing entanglement of two pieces of space [6], does not explain the driving force behind why two equal pieces of space, separated by less than two black holes, may form entangled pairs. The two-space piece entanglement approach describes entanglement of two space pieces in abstract space, while space does not have independent existence, and it changes only within space-time frame.

For description of entanglement of particles simultaneously in space and time, we need a proper structure of space-time frame. However, a description of events with the proper mathematical formulation involving dynamical space-time is the main problem associated with physical laws. As was shown by Merali [11], without knowing the origin of space-time, we will not understand physical laws. General relativity suggested the revolutionary idea that space-time and gravitational fields are the same, but an "Einstein's matter," which curves space-time, is an external entity and has no inner connection with the space-time frame. Another problem of general relativity is that its space-time has no background frame.

Rovelli's statement [12] that space-time has a relation to the electromagnetic field is the modification of general relativity. The main problem of the Rovelli's statement is that he describes quanta as an independent identity, which cannot live in space-time. Rovelli's statement did not reveal a mathematical formulation, and its quanta have no commutation with the space-time frame. The other problem of this 
approach is the locality of the quanta, while any particle or antiparticle cannot have independent existence.

According to Rovelli's opinion [12], there is no difference between a gravitational field and space-time, and the locality of a particle can be defined with respect to the gravitational field. This approach is similar to the Newtonian concept that acceleration has a meaning with respect to the gravitation field. However, any field, particularly a gravitational field, cannot have an independent existence; therefore the relation of locality to the gravitational field leads to the uncertainty in quantum mechanics.

Smolin [13] developed quantum field theory and suggested that at the Planck scale, space exists in the form of fundamental discrete units instead of general relativity's continuous space-time frame. But quantum field theory, similar to Newtonian physics, do not have space-time structure, which interacts with the event. The origin of discrete space and the condition of its independent existence is also not clear.

Therefore, different views on space-time and the absence of the origin of the background of space-time frame in both theories is the main problem for reconciling these theories into the unified theory. One of the main problems of these theories also is the locality of a particle in the space-time frame.

Einstein showed that space and time are simply different dimensions of the same space-time continuum. By his opinion, energy and momentum are the same quantities of space-time, which has four dimensions. The relative quantity of energy and momentum depends on the observer.

The problem of this approach is that the dynamical nature of the space-time variables connected within the continuum framework, which did not allow distinction of the local properties of time and space identities. General relativity determines the dynamics of matter by the geometry of space-time and does not explain the origin of the mass and energy, which curves the structure of space-time. The problem of Newtonian physics, regarding how the moving body responds to action in relativity theory, also remains an open question.

Unfortunately, the basic formulation of general relativity does not provide the answers to these questions. That is why the theory of relativity itself became the "observer" between Newton's physics and quantum mechanics.

It is necessary to note that problems of founding a unified theory are due to the problems of energy conservation, which is not complete in the theory of relativity and quantum physics. The approaches related to the generation of a unified theory do not use the principle of conservation of energy as the basis for the unification of relativity and quantum physics. The theory of relativity has a problem with the conservation of energy, which leads to the problem of singularity at small scales. Quantum mechanics suggests that particles borrow energy for some time and then return them. However, quantum mechanics does not explain the origin of this energy, which is borrowed and conserved in the wave function.

It is clear that for the generation of unified theory, we have to find a proper mathematical formulation of the conservation of energy, covering the higher scale space-time of relativity and the small-scale quanta of quantum mechanics. The model connecting relativity and quantum mechanics should involve the dynamic local state of space and time variables which, independent of the energy input, can operate between the small scale of quantum physics and large scale of relativity.

The known statements of Noether's theorem on conservation of energy, being philosophical in nature, are not applicable for generation of a mathematical formulation of the space-time picture of a particle.

Lagrange and Hamilton have suggested the conservation of energy in the form of differential equations, which is widely used in classical and quantum mechanics. 
The problem associated with these differential equations is that they describe the dynamical laws in abstract space with an independently moving interval of time.

Another principle, which is related to the conservation of energy, is the Lagrangian action principle. For the action integral to be well defined, the trajectory has to be determined simultaneously in time and space coordinates. However, the Lagrangian action principle does not cover these requirements.

Usually, the known mathematical formulations of dynamical laws either simplify space to conserve the details of time or simplify time to preserve the spatial dimension. The Lagrangian or Hamiltonian mechanics are the examples of such an approach, which was the reason for the replacement of differential equations of classical physics by Schrödinger's wave function.

To compose a unified theory, first, it is necessary to solve the locality problem of classical physics and quantum mechanics, which describe an event as a change of state of something without relation to something itself.

\section{Commutation of space-time with the principle of conservation of energy}

In our early studies [14-16], we suggested that the change of a function in relation to its local position $\left(\boldsymbol{\Delta} \mathbf{f} / \mathbf{f}_{\mathbf{1}}\right)$ could be a sufficient entity for the identification of change. The non-unitary function $\Delta \mathbf{f} / \mathbf{f}_{\mathbf{1}}$ with the fractional feature has a "quantum mechanical behavior": the classic operator in the form of $\Delta \mathbf{f} / \mathbf{f}_{\mathbf{1}}$ portion describes the fraction of the change (spinning or vibration) of a function around its dynamical initial locality to repeat its origin. Similarly, the operator $\Delta \mathbf{S} / \mathbf{S}_{1}$ describes the fluctuation of space with the applied force in relation to its origin, while the operator $\Delta \mathbf{t} / \mathbf{t}_{1}$ describes the fluctuation of time about instant of action.

In the conjugated space-time field frame, the position of a particle, localized within the space-time frame in relation to its origin, is not a point; it exists within a very certain discrete non-virtual space-time manifold, commuting dynamic energy, which is distributed within space and time fields. On this basis, the origin of spacetime is the energy, which generates space-time and holds its conservation within space and time phases.

In accordance with the above-described principle of conservation of energy within the space-time frame, the space-time becomes the resulting non-unitary inner product of energy distribution, which comprises the portions of energy consumed in space phase (event mass) and restored in time phase:

$$
\begin{gathered}
\frac{\frac{\Delta S}{S_{1}}}{\frac{\Delta t}{t_{1}}}=\frac{E_{a p}-E_{s}}{E_{s}} \\
\frac{\Delta S}{\Delta t}=\frac{S_{1}}{t_{1}}\left(\frac{E_{a p}}{E_{s}}-1\right) \\
\lambda=\frac{E_{\text {ap }}}{E_{s}}-1
\end{gathered}
$$

at $\lambda=1, \mathbf{E}_{\text {ap }}=2 \mathbf{E}_{\mathbf{s}}$.

$\mathrm{S}_{1}$ and $\mathbf{t}_{1}$ are the space and time variables corresponding to the dynamic local boundary; $\mathbf{E}_{\mathbf{a p}}$ and $\mathbf{E}_{\mathbf{s}}$ are the energies of action and under action systems of interaction at conditions corresponding to the local boundaries of $\mathbf{S}_{1}$ and $\mathbf{t}_{1}$. On this basis, the space and time phases, which "absorb" applied force and carry energy, attain features of an energetic field. The minimum portion of quanta generates an elementary space-time frame. 
The portion of energy conserved in space phase $\left(\mathbf{E}_{\mathbf{s}}\right)$ generates momentum of a particle with the mass, which curves the space-time frame to bring energy conservation to the initial state. Therefore, the space-time itself generates curvature to hold the conservation of energy within the boundary-mapped space-time frame. As Eq. (2) shows, the increase of $\mathbf{E}_{\mathbf{s}}$ and the reduction of $\left(\mathbf{E}_{\mathbf{a p}}-\mathbf{E}_{\mathbf{s}}\right)$ function gradually generate a curved space-time which in the form of gravitation returns an event to the initial state.

In accordance with Eq. (2), conservation of energy does not exist without localization in space-time frame, and the localization has to be non-uniform. It is easy to show that space and time are the resulting non-unitary portions of a non-uniform distribution of energy, consumed in space phase (forming mass) and restored in time phase:

In accordance with Eq. (2), the energy portion inserted to the space-time frame travels through wave of exchange interaction and determines the exact pathway of a particle. The right side of Eq. (2) describes the frequency of energy consumption in space by the matter particles, while the left side shows the frequency of the change of space and time wave fields. Following Einstein's approach that energy and momentum are the same quantities of space-time, we can show that the right side of Eq. (1) shows the ratio of available energy and momentum, commuted with the left side space-time frame. The entities $\Delta t, t_{1}$ and $\Delta S, S_{1}$ perform as the same identities of energy carrier, existing in the opposite phases. Equation (1) describes a mathematical formulation of the energy-momentum relation where space and time are the products of energy conservation and energy-momentum ingredients are the conjugated outcomes of space-time. Therefore, energy and momentum are same quantities as Einstein stated; moreover, space and time are the inner products of the energy-momentum conservation.

Now we may give specification of space, which is different from Caroll's [10] statement. We think that the relationship between space-time curvature and energy is the natural consequence of a non-uniform conservation of energy, which generates a space-time frame, carrying non-separable portions of boundarymapped energy.

We can now give specifications of entanglement based upon the conjugation of the space-time frame with the energy resources. The phenomenon called entanglement may appear only within conjugated space and time phases, carrying conservation of energy-momentum pairs though growth of the space-time frame.

Thus, any interaction of two different pieces of space (entanglement) in reality is the entanglement of space and time phases carrying out the conservation of energy through portions of energy, distributed within these phases. The space phase without entanglement with time cannot exist. The identity called space is the materialized portion of energy, while time destroys the material portion of energy, bringing an event to the initial state. When all the available energy is consumed in space $\left(\mathbf{E}_{\mathbf{a p}}=0\right)$, "space" particles decay to "time" antiparticles.

The entanglement of space and time phases as displayed in the form of gravitation appears with the accumulation of energy in space phase and generation of tendency to return space phase back to the background state.

The right side of Eq. (1) describes particle and antiparticle relations. Equation (2) treats the matter field through space phase, while the antimatter field is treated with the time phase. The coupling of these phases in space-time unit carries the non-uniform conservation of energy. Therefore, mass does not exist out of spacetime and does not appear by sudden spontaneous symmetry breaking; at zero mass of particles, energy is not conserved. 


\section{Matter-antimatter asymmetry}

The space-time symmetry, which may present matter-antimatter symmetry, is possible only when energy is equally distributed between two phases. In this case, "energy is not consumed and not destroyed." This is the timeless symmetry, which violates the classical principles of energy conservation that "energy is neither generated nor destroyed but rather is transformed from one state to another."

At the small scale, energy distributed in the small portion of the space phase in the form of mass is low, and energy is predominantly distributed in time phase ingredients, performing as antiparticles. This situation takes place in the case of a proton. The total mass of quarks, forming protons, is much smaller than the mass of protons.

We can now explain matter-antimatter asymmetry and the generation of more particles than antiparticles in a pair of collision experiments. To conserve energy, any action should lead to the consumption of energy $\left(\mathbf{E}_{\mathbf{a p}}<2 \mathbf{E}_{\mathbf{s}}\right)$ in order to eliminate infinite timeless symmetry. The condition $\mathbf{E}_{\mathbf{a p}}=\mathbf{2} \mathbf{E}_{\mathbf{s}}$ describes symmetry which is possible when two particles exist in symmetry with one antiparticle. This condition takes place only in proton-neutron pairs, which carry discrete symmetry of quarks with 1:2 relations.

In accordance with Eq. (2), due to the consumption of kinetic energy of collisions for the locality of generated particles, the energy carried by the produced particles is less than the energy required to restore particle-antiparticle symmetry.

The boundary-mapped space-time frame, involving a limitation on maximum velocity of the speed of light, is a requirement for energy conservation. Equation (2) presents the boundary of space-time by local position, dynamically growing in accordance with the available portion of energy. In a simple form, if there is a local position, there should be a boundary of the change of the energy, which carried by the space-time field.

The left side of Eq. (2) involves the dynamic conservation of the space-time frame as non-unitary "grains," while the right side shows the non-uniform conservation of the energy-momentum exchange relation, carrying the dynamic flux of the energy portion to the local $\mathbf{S}_{1} / \mathbf{t}_{1}$ metric of space-time frame. The gradient of energy in relation to the initial state $\left(\mathbf{E}_{\mathbf{a p}}-\mathbf{E}_{\mathbf{s}}\right) / \mathbf{E}_{\mathbf{s}}$ as an equivalent form of spacetime "grain" becomes the "non-unitary quanta" which describes the change of the local space-time frame as an exchange interaction of a particle with the applied force. The portion of energy, distributed in space and time phases, determines the strength of force and repulsive reaction (inertia) of matter.

It is easy to show that the non-uniform conservation of energy has to be the ground concept for the unification of relativity and quantum physics. Starting from the basic statement of general physics that energy is conserved through its conversion from one form to another, we arrive at the concept that a dynamical event of energy conversion has to have locality within finite space and time coordinates. In principle, the features of energy conservation during its conversion from one form to another are clear from Planck's theory of black body radiation, which changes the frequency of energy with radiation. The change of frequency of radiation is the result of non-uniform locality of energy within the space-time field.

The known statement of energy conservation that energy can neither be created nor destroyed but can only be transformed from one form to another does not involve the space-time frame of this transformation. Noether's theorem, describing energy and momentum conservation, separately also does not describe change of energy in space-time frame because time and space are not separable entities. 
The non-uniform conservation of energy leads to the collapse of the concepts on uniformly moving different reference frames in relation to which all-physical laws are valid. It is clear that even light cannot be the reference frame because light energy is non-uniformly conserved.

Eq. (1) shows that the space-time frame, which emerges from the non-uniform splitting of photons, is the fundamental building block of energy and matter: the space-time frame generates ordinary matter, which in energy-mass exchange interaction carries accelerated conservation of energy.

Within the non-uniform conservation of energy through the space-time frame, the unification of electromagnetism with the space-time frame becomes an obvious concept. The multiple $S_{1} / t_{1}\left(E_{a p} / E_{s}-1\right)$ of Eq. (2) is the localization of the electromagnetic field $\left(E_{a p} / E_{s}-1\right)$ within the local space-time $S_{1} / t_{1}$ frame. The local space-time $S_{1} / t_{1}$ metric undergoes change with the consumption of energy flux. The energy flux $\left(\mathbf{E}_{\mathbf{a p}} / \mathbf{E}_{\mathbf{s}}-1\right)$ is not uniform and presents the local energy portion, remaining from the exchange interaction. That is why electromagnetism is not Galilean invariant.

Equation (2), due to the involvement of the local frame of space-time and exchange energy-mass interaction, predicts the precise measurement of velocity and the local position of a particle; the exchange interaction eliminates the reference frame phenomenon, and different observers will have the same measurement if they have the same exchange interaction, coupled with the local position.

Equation (2) explains the uncertainty principle in a way that, in order to probe the small scale of space, we have to apply large amounts of energy. Probing the state of a particle to get information is possible only through an exchange interaction. At the Planck scale, there is no space-time frame, and the exchange interaction (2) is the reason why we cannot obtain any information and probe the state of a particle at Planck's scale. On this basis, by probing vacuum we can get information only on discrete uniform conservation of energy in the form of particles-antiparticles, breaking the symmetry with the formation of the space-time frame.

The condition, when the portion of energy conserved in space phase is equal to the portion of energy in time phase, could be considered as a uniform conservation of energy in the form of "Noether's symmetry." This condition corresponds to the relation:

$$
E_{a p}=2 E_{s}(2) \Delta S / S_{1}=\Delta t / t_{1}(3) \Delta S / \Delta t=S_{1} / t_{1}
$$

At the condition (3), the unlimited translation of energy portions between the opposite phases of space-time variables in the form of matter-antimatter fluctuations should lead to the "ultraviolet catastrophe." However, annihilation takes place within an asymmetric space-time frame; therefore, the non-uniform distribution energy moves in the direction of space expansion, which eliminates the ultraviolet catastrophe. On this basis, continuous uniform conservation of energy, matter-antimatter symmetry, and uniform continuous existence of any type of symmetry is impossible.

In symmetry, the space-time manifold of a particle after the change should look the same (4). But at $\mathbf{E}_{\mathbf{a p}}=\mathbf{2} \mathbf{E}_{\mathbf{s}}(2)$, the space and time fields are symmetrically interchangeable only in a discrete mode (2) where after the change the space-time frame holds the local state (4) only within the frame of discrete symmetry.

We think that the performance of the three particles of baryonic space-time $\mathbf{n}-\mathbf{p}$ matter in the form of boson-fermions relations follows this requirement. Therefore, without discrete performance of energy-mass exchange interaction in an elementary space-time unit, baryonic matter cannot exist in a symmetric manner. The strong and weak forces appear as the coupling product of exchange interaction in order to hold the discrete symmetry of the space-time frame of the baryonic matter. 
In accordance with the non-uniform conservation of energy, the spin as the space-time identity is the "face" of a particle. The particle may have identity of baryonic structure if it has the space-time frame in discrete symmetry at $\mathbf{E}_{\mathbf{a p}}=\mathbf{2} \mathbf{E}_{\mathbf{s}}$ with the participation of dynamic three jet particles.

Therefore, within the principles of non-uniform conservation of energy, light is not a uniformly moving reference frame. Light photons cannot exist without spacetime frame and, due to the moving within the non-uniform space and time phases, have features of electromagnetic wave.

The space-time, which has to carry conservation of energy, generates a nonvirtual local frame and moves it relative to the state of energy restoration.

The condition $\mathbf{E}_{\mathbf{a p}}=0$ of Eq. (2) is the background state of the discrete spacetime field where asymmetric space and time variables, for maintaining conservation cycles, undergo the discrete translation as the portions of energy in the different fields. At this state, all types of the interactions are discretely unified.

In accordance with Eq. (2), the gravitational field is the reverse phase of the electromagnetic field (negative energy solution), which restores energy at the origin. In the gravitational field, there is no space-time frame, while the electromagnetic field generates the space-time field and moves in the form of a wave through this frame. Due to the conservation of electromagnetic waves through the spacetime frame, it propagates through transverse waves, while gravity moves back to background state through a longitudinal wave.

It is necessary to note that it is not possible to get singularity-free quantization of space without a background space-time frame. The time parameter in quantum mechanics is an external entity, and quantum theories do not provide a dynamical space-time frame. The model in the form of Eq. (2) provides an entirely new function for the quantization of time.

Model (2) shows that energy appears as the inner product of the coupling of space and time fields (right-handed translation), and in reverse order, the origin of space-time variables is the decay of energy into virtual space and time entities (left-handed translation), with restoration of energy at the background state. This is the non-uniform, non-static conversion of energy from one form to another. On this basis, time appears as the product and boundary of the discrete non-Noetherian dynamic conservation of energy, carrying energy within the space-time frame. Due to the action-response interaction (2), we observe an event only in the past.

Model (2) describes a background-dependent space-time frame where the background state is not a fixed state but the dynamical origin of energy conservation cycles.

The space and time phases of energy conservation at the background Planck scale do not have a space-time frame; rather, they exist in the form of condensate without a shape. This approach is different from Wheeler's opinion that at Planck scale space and -time have a space-time foam [11].

According to Rovelli, [11] the state of the system may be certain when it has reference to a second physical system. In accordance with model (2), the second system is the applied force (energy) which generates the exchange interaction.

\section{Detailed features of the uncertainty principle of quantum mechanics}

Now based on model (2), we can explain details why the combination of momentum-position in quantum physics leads to uncertainty. Model (2) predicts that local position in the form of a point cannot give information about momentum or position, which can be relevant only to the exchange interaction with the applied 
force. Force and an event individually have no free existence, and they exist only through an exchange interaction. The exchange interaction generates velocity, which describes this interaction in the form of discrete packets showing how many times the reflected energy of exchange interactions repeats that interaction.

The uncertainty principle describes the commutation of momentum and position in the form, which does not hold conservation of energy. The uncertainty principle does not describe the change of position within the space-time frame and presents momentum without the energy-momentum exchange interaction.

When there is no applied energy $\left(\mathrm{E}_{\mathrm{ap}}=0\right)$, gravitational and inertial forces cancel each other, and a particle falls back to the initial state. This approach explains electromagnetic phenomenon, which has to be understood through interaction within two space-time frames.

Local position has conjugation with the force (energy) carrying particle which itself is a carrier of space-time. Therefore, a local position exists only through interaction with the force-carrying particle, which does not obey Lorentz symmetry.

Model (2) involves the exchange of interaction of energy portions in space-time instead of the curvature tensor of relativity. Inertia is not determined by mass itself because the mass of a particle has no independent existence.

Model (2) involves the energy-momentum exchange interaction and, similar to Maxwell's antisymmetric field tensor, describes antisymmetric energy distribution in the space-time field.

Particle physics connects the formation of mass with the breaking of symmetry, but symmetry, as is known from Neother's theorem, is associated with the conservation law. Therefore, breaking of symmetry has to be analyzed within the principles of energy conservation.

The model of non-uniform conservation of energy (2) involves the commutation of space-time and energy-momentum ingredients that explains symmetry breaking in the distribution of energy within the asymmetric boundaries of space and time phases.

It is obvious that non-uniform conservation of energy within an asymmetric space-time frame excludes the existence of continuous symmetry of particlesantiparticles, whereas continuous symmetry needs infinite energy resources to hold symmetry.

Model (8) connects space-time position with the energy-momentum exchange relation and shows that this relation within space-time boundary-mapped frame cannot be subject to uncertainty because position as a spatial variable does not have existence, independent of time.

\section{Principles of generation of mass and gravitation}

One of the main problems related to the generation of mass by spontaneous breakdown of continuous symmetry, given by the Higgs mechanism, is that this mechanism does not connect the generation of mass with the space-time locality of a particle and does not explain why background continuous symmetry has to be broken in an unnatural way. The mechanism of mass generation also has to explain why collision experiments produce more matter particles than antimatter particles.

In this chapter, we will discuss how the non-uniform energy conservation concept is an alternative mechanism of mass generation. The non-uniform distribution of energy portions within asymmetric space and time phases requires generation of fields with different energetic properties (frequency and amplitude) which is the only way for carrying conservation of energy through these fields. The coupling of 
two fields with different energetic properties as energy consuming and energy restoring phases generates the non-virtual space-time frame, which appears to be the non-uniform conservation of energy through energy-mass exchange transformations $\left(\mathrm{E}_{\mathrm{ap}} / \mathrm{E}_{\mathrm{s}}-1\right)$.

The background state of a space-time frame is the relation of virtual asymmetric space and time phases, which proceeds through the conversion of energy from one form to another (8), through the translation of asymmetric entities, such as $\Delta \mathbf{S} / \mathbf{S}_{1}$, $\Delta \mathbf{t} / \mathbf{t}_{1}$, carrying energy portions as virtual matter and antimatter particles.

We can describe the non-uniform background energy-mass translation by conversion of light photons to electron/positron pairs, which is a well-known quantum mechanics translation event. Quantum mechanics states that during this translation, energy conservation is held by fluctuations, such as particles that borrow energy and return it after a very short time:

$$
\boldsymbol{\gamma} / \boldsymbol{\gamma}=\mathbf{e}^{+} / \mathbf{e}^{-}
$$

The energy-matter translation given by Eq. (5) does not count the time phase of energy conservation and the locality of the produced particles, while the translation between photons and leptons takes place in abstract space. Equation (5) could be the discrete translation of energy in the form of infinite fluctuations of the background quantum state. It is clear that in this case there is no natural way for breaking of the continuous symmetry of discrete fluctuations, forming timeindependent infinite symmetry of matter-antimatter relations. Equation (5) does not reflect the borrowed time in the change of energy.

Conservation of energy requires a certain finite frame for locality, therefore space and time cannot exist as separate variables. The formation of a particle within any time scale without locality in space phase leads to nonconservation of energy.

On this basis, we replaced Eq. (5) with the relation:

$$
\boldsymbol{\gamma} / \boldsymbol{\gamma}=-\left(\mathbf{e}^{+} / \mathbf{e}^{-}+\nu_{\mathbf{e}} / \boldsymbol{\nu}^{-}\right)
$$

The right side of Eq. (6) involves an additional identity in the form of neutrinos to cover the missing part of energy conservation in a time-dependent frame. Equation (6) represents the mechanism of energy conservation, which involves the decay of energy into asymmetric space and time field particles (2), characterized by different energy densities. Conversion of light photons from one form to another for conservation needs the generation of phase differences, which appears with the formation of $\mathrm{e}^{+} / \mathbf{e}^{-}+\nu_{\mathrm{e}} / \boldsymbol{\nu}_{\mathrm{e}}^{-}$pairs.

The space field particles, comprising $\mathbf{e}^{-} / \mathbf{e}^{+}$pairs, have more energy density, while time phase particles, comprising $\nu_{\mathrm{e}} / \nu_{\mathbf{e}}^{-}$pairs, have energy portions of a high frequency. It is precisely for this reason that the mass for neutrinos is significantly less than that of electrons. The right-handed antineutrino and left-handed neutrino pair together with the electron/positron pair represents the distribution of energy within virtual space and time phases. Due to the locality within space, close to Planck's size, the performance of virtual matter particles became time-dependent, and it attains a velocity less than the speed of light photons. Hence, the parity translation (6) became non-invariant.

Generation of $\mathbf{e}^{-} / \mathbf{e}^{+}+\nu_{\mathrm{e}} / \boldsymbol{\nu}_{\mathbf{e}}^{-}$particles (6) is the translation of the energy of photons to virtual space and time phase particles which could be specified as "empty space" particles. The "empty space" is the medium where $\mathbf{e}^{-} / \mathbf{e}^{+}+\boldsymbol{\nu}_{\mathrm{e}} / \boldsymbol{\nu}_{\mathbf{e}}{ }^{-}$ particles form a fluid with a continuum spectrum. In the absence of energy flux $\left(\mathbf{E}_{\mathbf{a p}}=0\right)$, a loss of the space frame takes place with the translation of virtual particles back to photons. However, particles before giving the "borrowed' energy 
back lose localization in space phase and lose some portion of the energy which has to go in parallel with the absorption of photons by $\mathbf{e}^{-} / \mathbf{e}^{+}$pairs. This phenomenon is the main feature of energy nonconservation during the return of "borrowed" energy of quantum fluctuations. Generation of space phase and distribution of energy in the space field leads to the non-uniform conservation of energy in space by absorption of photons by $\mathbf{e}^{-} / \mathbf{e}^{+}$pairs with the formation of pairs of heavy bosons.

\section{The Higgs mechanism}

The question as to why particles need mass is the main question of fundamental physics. Based on the Standard Model, the generation of mass by particles is due to the spontaneous breaking of symmetry within pairs of particles-antiparticles in the universe. The Standard Model applies Higgs mechanism in order to explain the phenomenon of spontaneous symmetry breaking.

In accordance with the Higgs mechanism, space is filled with a field, and when the weak force particles (electron, quarks) move through Higgs field, they gain mass. However, the Higgs field does not give mass to protons, which are generated from quarks. The Higgs mechanism does not explain why photons are not given a mass when they pass through the Higgs field.

It is necessary to note that particle physics news involves hot discussions surrounding the discovery of Higgs bosons. The Higgs mechanism does not explain why field suddenly shifted matter-antimatter symmetry to a mass generation event. Many other questions also remain open, such as where the Higgs field came from and why the universe should contain the Higgs field for creating matter-antimatter asymmetry.

Based on the non-uniform conservation of energy, we can explain why conversion of kinetic energy, produced from collision of matter-antimatter pairs, breaks the symmetry. Model (2) shows that conservation of energy gives associated mass which is localized in the space-time frame in order to eliminate singularity in energy conservation. From model (2), it also follows that the zero value of the field takes place at $\mathbf{E}_{\mathbf{a p}}=0$, which leads to ripples between space and time phases in the socalled Higgs Field.

Model (2) shows that at $\mathbf{E}_{\mathbf{a p}}=0$ all energy is accumulated in the form of mass, and the space-time frame decays to virtual space and time ingredients. Virtual space and time ingredients annihilate each other as ripples of particles and antiparticles with the generation of gravitational energy accelerating to the background state with the longitudinal wave. The background space does not go to the zero value because in this case the energy of the universe will be infinite. At $\mathbf{E}_{\mathbf{a p}}=0$, the radioactive decay of the space-time frame produces heavy bosons $\mathbf{W}^{+}, \mathbf{W}^{-}$and $\mathbf{Z}$. At minimum space, the energy accumulated in time phase generates location of $\mathbf{W}^{+}$, $\mathbf{W}^{-}$bosons in quarks with generation of space-time frame of matter.

When all the available energy is consumed in space $\left(\mathbf{E}_{\mathbf{a p}}=0\right)$, the background space (Planck scale) with the lowest valley of energy becomes the vacuum state and generates gravitational attraction toward all the ingredients of decay of space-time frame to the background state.

Coupling of gravitational energy with virtual space and time leads to the regeneration of non-virtual space-time frame of matter.

The two parts of space, carrying different contents of energy, have entanglement, but they have no symmetry and are not two identical, equal halves, which 
was suggested by the authors of the EP paper [6]. The widely separated regions of space carry non-uniform conservation of energy within the space-time frame; therefore, the entanglement of nonidentical pieces of space is due to the connection of energy within space and time phases regardless of space and time scales.

In accordance with our concept of the non-uniform conservation of energy principle, the phenomenon called mass generation is the requirement of energy conservation. Based on model (2), generation of mass is not a spontaneous symmetry-breaking event; it is the requirement of energy conservation that is carried through a discrete non-uniform space-time frame. The space and time parameters work as particles and antiparticles carrying energy conservation through their integrated frame.

Model (2) describes the conjugation of force $\left(\mathbf{E}_{\mathbf{a p}}\right)$ and matter (Es) particles, forming the space-time frame of a matter. On this basis, light energy can be observable only if it reflected from the space-time frame of matter. The forcecarrying particle $\left(\mathbf{E}_{\mathbf{a p}}\right)$ generates its conjugated particle $\left(\mathbf{E}_{\mathbf{s}}\right)$ that has a mass, simultaneously conserving energy and momentum. The model shows that when the corresponding particle has no mass, the conservation of energy diverges to infinity.

Model (2) may explain why photons do not attain mass when they pass through Higgs field. Model (2) comprises of massless ( $\mathbf{E}_{\mathbf{a p}}$ ) and mass-containing particles $\left(\mathbf{E}_{\mathbf{s}}\right)$. At $\mathbf{E}_{\mathrm{ap}}=0$, particles which form the so-called Higgs field have no space-time configuration. Assuming information that the Higgs boson may decay to a pair of photons, we can describe the generation of Higgs field particles and photons through Eqs. (7) and (8):

$$
\begin{gathered}
\mathbf{e}^{+} / \nu_{\mathbf{e}}+\mathbf{e}^{-} / \nu_{\mathbf{e}}{ }^{-}=\mathbf{e}^{+} / \mathbf{e}^{-}+\boldsymbol{\nu}_{\mathbf{e}} / \boldsymbol{\nu}_{\mathbf{e}}{ }^{-} \\
\mathbf{e}^{+} / \boldsymbol{\nu}_{\mathbf{e}}+\mathbf{e}^{-} / \boldsymbol{\nu}_{\mathbf{e}}^{-}=-\mathbf{e}^{+} / \mathbf{e}^{-}+\boldsymbol{\nu}_{\mathbf{e}} / \boldsymbol{\nu}_{\mathbf{e}}{ }^{-}=2 \mathbf{Y}
\end{gathered}
$$

Scheme (8) describes the generation of light photons from dark matter. Due to the consumption of light photons for generation of space-time frame of observable matter, the conversion reaction (8) does not have the same velocity in both directions and is, therefore, not a time reversal invariant process.

Now the question is how we can describe the mass of protons, which does not originate from Higgs bosons. By Standard Model, the mass of protons comes from binding energy-gluons.

The coupling of energetic field with the virtual particles $\mathrm{e}^{+} / \mathbf{e}^{-}+\nu_{\mathrm{e}} / \nu_{\mathbf{e}}^{-}$leads to the insertion of energy to the virtual particles with the generation of quarks and space-time frame:

$$
2 \mathbf{Y}+\mathbf{e}^{+} / \mathbf{e}^{-}+\nu_{\mathbf{e}} / \nu_{\mathbf{e}}^{-}=\mathbf{e}^{+} / \nu_{\mathbf{e}}+\mathbf{e}^{-} / \nu_{\mathbf{e}}^{-}
$$

The coupling reaction (9) maintains the conservation of energy and momentum within the boundary-mapped space-time frame.

The virtual particles $\mathbf{e}^{+} / \mathbf{e}^{-}+\nu_{\mathbf{e}} / \nu_{\mathbf{e}}^{-}$exist in the form of dark matter and became observable only after the generation of the space-time frame. Similarly, the photons, generated from decomposition of matter space-time frame, exist in the form of dark energy and become observable only after interaction with the space-time frame of matter.

Therefore, matter and energy are observable only within interactions with the space-time frame. Interaction of virtual particles with the energy photons is such a coupling of virtual particles with themselves. These interactions follow the scheme, which is shown through the conversion (10): 


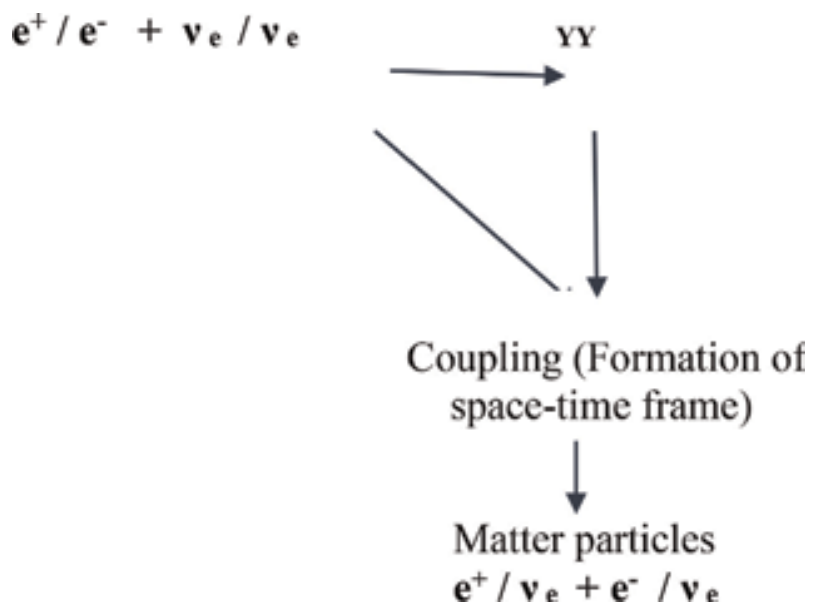

We may present the scheme (10) in the reverse order:

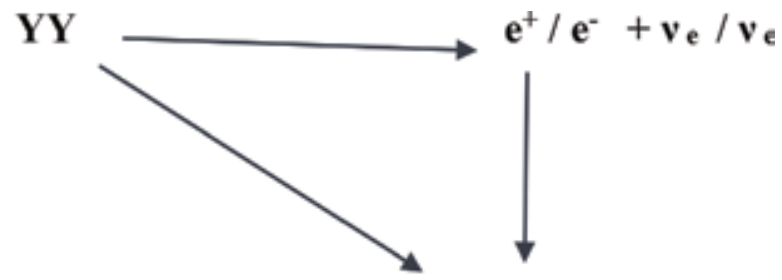

\section{Coupling (Formation of space-time frame)}

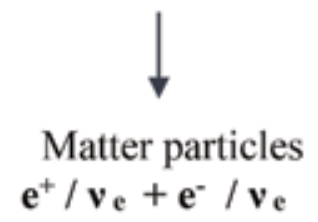

Model (2) explains the invariance of time reversal transformation in scheme (10). The energy of space-time matter $\mathbf{E}_{\mathbf{s}}$ is always less than the energy of photon particles. The process moves in the direction of expansion of the space-time frame of observable matter.

Scheme (11) in a similar way explains the generation of matter. Light photons transform to ingredients of matter particles, such as electron/positron and neutrino pairs. Due to the consumption of light energy during the coupling of photons with the ingredients of matter particles, the conversion of light photons is not invariant to the reversal of time.

\section{The energy-mass equivalence}

In accordance with the theory of relativity, mass and energy are different manifestations of the same identity, and the equivalence of mass and energy is a consequence of the symmetries of space and time [17]. This statement of the theory of 
relativity does not explain when the difference between mass and energy disappears and when energy and mass became different entities.

Based on model (2), the mass-energy equivalence is the consequence of energy conservation, which is carried through antisymmetric space-time phases. When there is no available energy to be conserved within space-time, the difference between energy and mass disappears. The difference between energy and mass appears when there is an energy (electromagnetic, kinetic) applied to space-time frame of a system. Production of energy in proton-proton collision experiments leads to the separation of mass from energy with the generation of a space-time frame of the produced particles.

It is necessary to show that the energy-momentum exchange interaction, which generates mass, is similar to Lorentz's theory of the generation of electron's mass. Lorentz connected mass with the electromagnetic effect and suggested that "back reaction of electric and magnetic fields leads to the generation of mass" [18]. However, Lorentz did not explain the nature of back reaction of electric and magnetic fields. Lorentz's formulation does not involve energy-momentum exchange interaction.

Lorentz's theory of electron mass is a very important approach for understanding the mass problem. Model (2) explains Lorentz's statement on the nature of apparent and true masses [18]. Model (2) shows that the motion of any body, for example, an electron, takes place with the consumption of energy, which is imported to the space-time frame. Due to the correlation of the mass with the applied energy, we measure only the apparent mass but never the true mass.

When $\mathbf{E}_{\mathbf{a p}}$ of model (2) is zero, space-time decays and moves back to the background state. In this case, the local energy portion responsible for the local state disappears. In accordance with model (2), when space-time frame undergoes decay $\left(\mathbf{E}_{\mathbf{a p}}=0\right)$, the difference between space and time variables disappears which generates a condensate field where the space and time portion of energy are uniformly mixed. Separation of space and time variables from the background condensate and the formation of space-time frame consume energy, while merging them restores that energy at the initial state. Therefore, energy is conserved only through the non-uniform distribution within the boundary-mapped space-time frame and the energy-momentum exchange interaction within the space-time frame.

At the background state, all available energy of the universe, accumulated within minimum space, leads to the separation of space and time portions of energy from condensate with the formation of space-time frame with asymmetric boundaries. Energy level in the non-uniform conservation stage is less than the energy of background condensate of uniform energy conservation stage.

Background vacuum and black hole are the boundary conditions of space-time frame and energy conservation. Due to the exchange interaction, black hole space has a strong gravitational effect toward electromagnetic energy and consumes all the available portion of energy. Vacuum is the other extreme of space-time and has strong gravitational effect to the portion of energy consumed in space. Therefore, electromagnetism and gravitation are two phases which transform into each other, satisfying the non-uniform conservation of energy. On this basis, the phenomenon called mass in the form of inertia appears as the carrier of energy conservation within an asymmetric space-time frame.

Due to the absence of space-time frame, a gravitational field in the form of a longitudinal wave does not react with the matter. On this basis, gravity has no discrete behavior, and there is no quantization of this field.

It is necessary to note that the combination of electromagnetism and gravity is not enough to complete the unified theory. In accordance with the concept of 
conservation of energy and momentum within the space-time frame, a complete unified theory requires the combination of electromagnetism and gravity with the space-time frame.

Therefore, our approach shows that description of space-time cannot be more fundamental than energy conservation, but the combination of space-time with the conservation of energy becomes a fundamental theory of reality.

Model (2) does not describe a particle as an entity, which is localized in space and evolves in time, but it presents a particle and space-time as a resulting quantity produced from each other.

Decomposition of a space-time frame $\left(\mathbf{E}_{\text {ap }}=0\right)$ leads to the loss of information which is why black hole radiation does not carry any information. Black hole radiation leads to the restoration of energy at the initial state and the starting of new information, carried by the new space-time frame.

In Eq. (2), the $\mathbf{E}_{\mathbf{s}}$ in the denominator is the dark matter, but in the numerator is the dark energy. When $\mathbf{E}_{\text {ap }}=0$, all the photon energy transforms to dark energy which disappears between dark energy and dark matter.

The exchange interaction of space and time portions in space-time frame leads to the formation of electric and magnetic fields with the generation of time translational non-invariant electromagnetic energy. The $\mathbf{E}_{\mathbf{a p}}-\mathbf{E}_{\mathbf{s}}$ parameter of the equation presents the vector potential of electromagnetism, which vanishes due to the consumption in space.

Therefore, electromagnetic energy cannot exist without discrete space-time frame and exchange interactions. Light is observable only through the reflection from space-time frame, and without space-time frame energy is dark. Higgs field has no space-time frame, which is why it is a scalar field. Quantum excitation is the redistribution of energy portions between space and time phases.

By Bose-Einstein statistics, two bosons with identical properties can be in the same place at the same time. Based on model (2) bosons have no space-time frame, which is why there is no difference between light bosons. Interaction of light bosons with the space-time frame and absorption of light with the growth of the matter space-time structure make light observable.

Model (5) explains the phenomenon called "nonlocality." The function $\mathbf{E}_{\mathbf{a p}}-\mathbf{E}_{\mathbf{s}} / \mathbf{E}_{\mathbf{s}}$ of model (2) which describes action-response parity is the origin of local action. At $\mathbf{E}_{\mathbf{a p}}=0$, a particle has no space-time frame and has no certain locality. When a particle has no space-time $\left(\mathbf{E}_{\mathbf{a p}}=0\right)$, all particles are the nondistinguishable ingredients of antimatter "condensate."

At $\mathbf{E}_{\mathbf{a p}}-\mathbf{E}_{\mathbf{s}}>0$ a particle has its own space-time frame and, therefore, independent locality. The condition $\mathbf{E}_{\mathbf{a p}}=0$ eliminates the action-response behavior of a particle which loses spin and moves to the background state. Therefore, energy conservation comprises of two steps: the decomposition of matter to an energetic field and the reverse process of transfer of an energetic field to matter.

In accordance with model (2), particles have space-time existence at positive energy state and have momentum in the opposite direction. The positive energy solution alone does not complete energy conservation. In accordance with our approach, the relationship between curvature of space-time and energy is the natural consequence of cyclic energy conservation within boundary-mapped spacetime.

In accordance with model (2), the positive and negative energy states are not symmetric. The negative energy state is the second portion of energy, conserved in the form of Higgs field. At the state of zero positive energy $\left(\mathbf{E}_{\mathbf{a}}=0\right)$, particles do not follow Pauli's exclusion principle and, thus, have no local space-time position; in other words, all the particles occupy the same position. 
Dirac analyzed the relation of energy and momentum-using equations, which involves the sum of these parameters. Model (2) suggests a different approach and involves the ratio of the available portion of energy and the energy, which is consumed by momentum, which eliminates the divergence of energy to infinity.

Now returning to Rovelli's statement [12] on relation of space to the electromagnetic field, we may add that space-time itself through energy distribution is generated from an electromagnetic field and as a medium carries an electromagnetic field. Merging of energy quanta with the generated space-time leads to the expansion of space of an event.

\section{Conclusion}

In this paper, we extended our analysis on the nature of space-time to give an input to the hot disputes on the generation of a unified theory by entanglement of two equal pieces of space through "Wormhole Bridge" which was the outcome of the ER and EPR papers. We suggest that the unification of the theory of relativity with quantum mechanics is possible only through the proper mathematical formulation of the law of conservation of energy within the space-time frame. In accordance with this approach, the conversion of energy from one form to another requires a non-separable space locality and time frame which generates a boundarymapped space-time structure wherein the consumption of light photons generates identities, such as space and time particles, carrying distribution of energy within the emerging non-uniform space-time field.

The non-uniform conservation of energy within the boundary-mapped spacetime manifold leads to the generation of a deterministic dynamical law. This concept unifies all the interactions of nature within the asymmetric space-time manifold, carrying the non-uniform conservation of energy through coupling of energy-momentum conservation frames. It also describes the origin of mass, as the product of the non-uniform conservation of energy within the non-invariant energy-mass relation. We showed that gravity is materialized in space energy, while time at zero energy state $\left(\mathbf{E}_{\mathbf{a p}}=0\right)$ in the form of ripples of space and time phases returns an event to the initial background state with restoration of a new elementary space-time frame. Based on the energy conservation model (2), at matterantimatter symmetry, the difference between energy and momentum disappears which destroys the space-time frame of the universe.

When energy is consumed by a space-time frame or by $\mathbf{e}^{-} / \mathbf{v}^{-}+\mathbf{e}^{+} / \mathbf{v}$ particles with the formation of quarks, the space-time frame returns to its origin after consumption of all the available portion of energy. Only energy, accumulated at background state, may separate $\mathbf{e}^{-} / \mathbf{e}^{+}+\mathbf{v} / \mathbf{v}$ dark field particle pairs and generate quarks. When the matter has no space-time frame, it is not observable. The dark matter and dark energy are "dark" due to the absence of space-time frame, and energy, which is produced from the decomposition of the space-time frame, is not observable. The dark energy becomes an electromagnetic energy when it interacts with the space-time frame. 


\section{Author details}

Agaddin Mamedov

Houston Technology Center, Sugar Land, Texas, USA

*Address all correspondence to: amamedov@sabic.com

\section{IntechOpen}

(C) 2019 The Author(s). Licensee IntechOpen. This chapter is distributed under the terms of the Creative Commons Attribution License (http://creativecommons.org/licenses/ by/3.0), which permits unrestricted use, distribution, and reproduction in any medium, provided the original work is properly cited. (cc) BY 
The Hot Disputes Related to the Generation of a Unified Theory Combining the Outcomes... DOI: http://dx.doi.org/10.5772/intechopen.88722

\section{References}

[1] Crew B. Linking General Relativity and Quantum Mechanics with Wormholes. International science publisher. USA: Science Alert; 2016

[2] Maldacena J, Susskind L. Cool horizons for entangled black holes. arXiv: 1306.05332v2 [hep-th]. 2013

[3] Susskind L. Copenhagen vs Everett, Teleportation and ER=EPR. arXiv: 1604.02589 v2 [hep-th]. 2016

[4] Cole B. Wormholes Could Be the Key to Beating The Heisenberg's Uncertainty Principle. USA, Science Alerts: International Science Publisher; 2016

[5] Grant A. Entanglement: Gravity's Long-distance Connection. Science News. USA: Independent Publisher; 2015

[6] Einstein A, Rosen N. The particle problem in the general theory of relativity. Physical Review. 1935;1:48

[7] Einstein A, Podolsky B, Rosen N. Podolsky; "Can quantum-mechanical description of physical reality be considered complete?". Physics Review. 1935;47(10):777-780

[8] Siegfried T. A new "Einstein" equation suggest wormholes hold key to quantum gravity. Science news. 16 Aug 2016

[9] Van Raamsdonk M. General Relative Gravity. 2010;42:2323-2329

[10] Cao CJ, Sean M, Carroll I, Michalakis S. Space from Hilbert Space: Recovering Geometry from Bulk Entanglement. arXiv: 1606.08444v3 [hep-th]. 2016

[11] Merali Z. Theoretical physics: The origins of space and time. Nature. 2013; 500:516-519
[12] Rovelli C. Quantum Gravity. Cambridge Monographs on Mathematical Physics. Cambridge: University Press; 2007

[13] Smolin L. The Road to Quantum Gravity. New York: Basic Books; 2001

[14] Mamedov AK. Unification of dynamical laws through frequency based discrete space-time and symmetry principle. European Journal of Scientific Research. 2010;42(3):359-384

[15] Mamedov AK. The Concept of Mass Based on Accelerated Conservation of Energy Within Asymmetric Space-time Phases. Rijeka, Croatia: Intech; 2018

[16] Mamedov AK. Unification of quantum mechanics and relativity based on discrete conservation of energy. In: The Selected Topics of Quantum Mechanics. Rijeka, Croatia: InTech; 2014

[17] Wikipedia.org/mass-energy equivalence

[18] Lorentz HA. The Theory of Electrons and the Propagation of Light. Nobel Speech. Stockholm; 1902 


\section{Edited by Francisco Bulnes, Vasilios N. Stavrou, Oleg Morozov and Anton V. Bourdine}

Research in quantum communication requires knowledge of the information theory and teleportation of information in any physical form using the quantum field as media of all different processes and aspects on the managing of their information, their boson processing and their technologies. Likewise, one of the most important principles in researching is the quantum intertwining to define the management of communication of the quantum type. Other aspects related to the design of technologies to the quantum communication base and their development on transceptor models of Bose-Einstein observation nature to form a quantum laser wave of atoms. This quantum wave is a wave-links chain that defines and determines the quantum communication. Quantum computing models are very useful to establish the programming order required in the quantum communication processes. The present book is a compilation of the chapters

that cover several of these themes and in other cases, put the field theories to test regarding what is quantum communication and its technologies. 